\title{
Local invertibility in Sobolev spaces with applications to nematic elastomers and magnetoelasticity
}

\author{
Marco Barchiesi, Duvan Henao and Carlos Mora-Corral
}

February 8, 2017

\begin{abstract}
We define a class of deformations in $W^{1, p}\left(\Omega, \mathbb{R}^{n}\right), p>n-1$, with positive Jacobian that do not exhibit cavitation. We characterize that class in terms of the non-negativity of the topological degree and the equality Det $=\operatorname{det}$ (that the distributional determinant coincides with the pointwise determinant of the gradient). Maps in this class are shown to satisfy a property of weak monotonicity, and, as a consequence, they enjoy an extra degree of regularity. We also prove that these deformations are locally invertible; moreover, the neighbourhood of invertibility is stable along a weak convergent sequence in $W^{1, p}$, and the sequence of local inverses converges to the local inverse. We use those features to show weak lower semicontinuity of functionals defined in the deformed configuration and functionals involving composition of maps. We apply those results to prove existence of minimizers in some models for nematic elastomers and magnetoelasticity.
\end{abstract}

\section{Contents}

1 Introduction

2 Notation and preliminary results 4

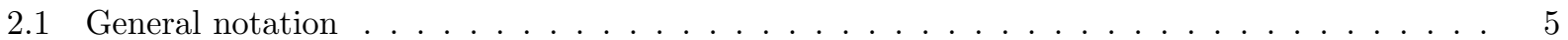

2.2 Approximate continuity and approximate differentiability $\ldots \ldots \ldots \ldots \ldots \ldots$

2.3 Area formulas and geometric image . . . . . . . . . . . . . . . . . . . . 7

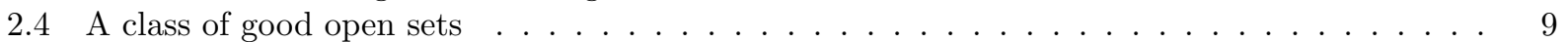

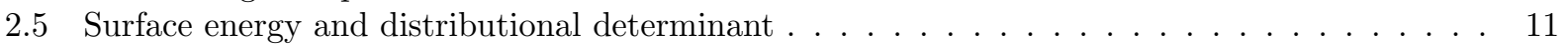

3 Degree for Sobolev maps $\quad 12$

4 Local invertibility and preservation of orientation $\quad 14$

$5 \quad$ Fine properties of deformations in $\mathcal{A}_{p} \quad 23$

5.1 Condition INV and Sobolev regularity of the inverse $\ldots \ldots \ldots \ldots \ldots$

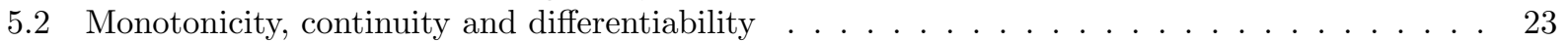

$5.3 \mathcal{H}^{n-1}$-continuity of the local inverse $\ldots \ldots \ldots \ldots \ldots \ldots \ldots \ldots \ldots$

5.4 The topological image $\ldots \ldots \ldots \ldots \ldots \ldots$

6 Stability of the inverse $\quad 32$

7 Lower semicontinuity $\quad 36$

7.1 Quasiconvex functionals in the deformed configuration . . . . . . . . . . . . . . 36

7.2 Div-quasiconvex functionals under incompressibility . . . . . . . . . . . . . . . 42

7.3 Polyconvex functionals involving a composition of maps . . . . . . . . . . . . . . 43 
8.1 A Frank energy model for nematic elastomers . . . . . . . . . . . . . . . . . . . . . . 46

8.2 A Landau-de Gennes energy model for nematic elastomers . . . . . . . . . . . . . . . . . . . . 48

8.3 Magnetoelasticity . . . . . . . . . . . . . . . . . . . . . . 51

\section{Introduction}

In this paper we prove a local invertibility result for Sobolev deformations in $W^{1, p}\left(\Omega, \mathbb{R}^{n}\right)$ with $p>n-1$ and $\Omega \subset \mathbb{R}^{n}$. Our motivation comes, on the one hand, from the need of defining a suitable subclass of deformations in $W^{1, p}$ that are regular enough so as not to exhibit cavitation, and, on the other hand, to prove well-posedness of variational models, like those for nematic elastomers and magnetoelasticity, that involve both reference and deformed configurations.

In the context of nonlinear elasticity, results on global invertibility of deformations can be found in $[7,15,70,71,63,61,16,42,43,44,45,46]$, whereas [32] deals with local invertibility. Most approaches use the topological degree as an essential tool. Among the papers cited before, we explain some of the ideas of Müller \& Spector [61], since their analysis has been instrumental in our work.

In their study of cavitation in solids (that is, the sudden formation of voids in materials subjected to a large tension), a key idea of [61] was the introduction of condition INV (see Definition 3.5), which is a topological condition that, together with the assumption $\operatorname{det} D \mathbf{u}>0$ almost everywhere (henceforth abbreviated as a.e.), implies invertibility a.e. and provides a sensible notion of orientation preserving Sobolev functions. Here $\mathbf{u}: \Omega \rightarrow \mathbb{R}^{n}$ is the deformation. They also showed that the distributional determinant Det $D \mathbf{u}$ (see Definition 2.26) is able to detect the process of cavitation. In this paper, even though we will deal with maps that do not present cavitation, we need to have a prior understanding of the mathematical conditions for cavitation precisely in order to define a class of functions that excludes it. In particular, they showed that, under natural assumptions (notably, condition INV), the equality $\operatorname{Det} D \mathbf{u}=\operatorname{det} D \mathbf{u}$ (meaning that the distributional determinant can be identified with the $L^{1}$ function $\operatorname{det} D \mathbf{u}$ ) means exactly that $\mathbf{u}$ does not exhibit cavitation. As a matter of fact, condition $\operatorname{Det} D \mathbf{u}=\operatorname{det} D \mathbf{u}$ has been traditionally an intermediate step to prove existence of minimizers in nonlinear elasticity (see $[6,60,63]$ and their many generalizations). However, in [44] an example was given of a one-to-one a.e. deformation $\mathbf{u}$ that creates a cavity even though $\operatorname{det} D \mathbf{u}>0$ a.e. and $\operatorname{Det} D \mathbf{u}=\operatorname{det} D \mathbf{u}$. That function does not satisfy condition INV and, in fact, the topological degree takes both positive and negative values. This raises the question of an adequate definition of orientation preserving Sobolev maps. While the traditional analytical definition is that $\operatorname{det} D \mathbf{u}>0$ a.e. (or $\operatorname{det} D \mathbf{u} \geq 0$ a.e.), it is more appropriate to use the topological definition stating that the degree is nonnegative: $\operatorname{deg}(\mathbf{u}, U, \cdot) \geq 0$ a.e. for any open $U \subset \Omega$ for which the degree of $\mathbf{u}$ in $U$ is defined (see Section 3). We will return to this issue later, notably in the statement of Theorem 1.1.

In [45] it was proven that, under the conditions of [61], the inverse function $\mathbf{u}^{-1}$ is also Sobolev (see [70] for an earlier related result). Hence, as a by-product of the theory of [61], we may state that the class of functions $\mathbf{u}$ satisfying INV, $\operatorname{det} D \mathbf{u}>0$ a.e. and $\operatorname{Det} D \mathbf{u}=\operatorname{det} D \mathbf{u}$ is, possibly, the right analogue in $W^{1, p}$ to the concept of orientation-preserving homeomorphism.

Condition Det $D \mathbf{u}=\operatorname{det} D \mathbf{u}$ can be expressed as

$$
\operatorname{det} D \mathbf{u}(\mathbf{x})=\frac{1}{n} \operatorname{Div}[\operatorname{adj} D \mathbf{u}(\mathbf{x}) \mathbf{u}(\mathbf{x})],
$$

where Div in the right-hand side denotes the distributional divergence. For smooth maps u, equality (1.1) is a consequence of Piola's identity, and so is the following variant, introduced in [59,60,63] (see also [70, Eq. (8)]):

$$
\operatorname{div} \mathbf{g}(\mathbf{u}(\mathbf{x})) \operatorname{det} D \mathbf{u}(\mathbf{x})=\operatorname{Div}[\operatorname{adj} D \mathbf{u}(\mathbf{x}) \mathbf{g}(\mathbf{u}(\mathbf{x}))]
$$

for all $\mathbf{g} \in C^{1}\left(\mathbb{R}^{n}, \mathbb{R}^{n}\right) \cap W^{1, \infty}\left(\mathbb{R}^{n}, \mathbb{R}^{n}\right)$. Obviously, (1.1) follows from (1.2) by taking $\mathbf{g}=\frac{1}{n} \mathbf{i d}$, but the 
example of [44] shows that the two conditions are not equivalent. In [42] it was introduced the energy

$$
\mathcal{E}(\mathbf{u}):=\sup _{\substack{\mathbf{f} \in C_{c}^{1}\left(\Omega \times \mathbb{R}^{n}, \mathbb{R}^{n}\right) \\\|\mathbf{f}\|_{\infty} \leq 1}} \int_{\Omega}[\operatorname{cof} \nabla \mathbf{u}(\mathbf{x}) \cdot D \mathbf{f}(\mathbf{x}, \mathbf{u}(\mathbf{x}))+\operatorname{det} \nabla \mathbf{u}(\mathbf{x}) \operatorname{div} \mathbf{f}(\mathbf{x}, \mathbf{u}(\mathbf{x}))] \mathrm{d} \mathbf{x},
$$

which measures the new surface in the deformed configuration created by $\mathbf{u}$ (for example, by the process of cavitation), and quantifies the failure of the equality (1.2). For the purpose of this paper, we just need to know that $\mathcal{E}(\mathbf{u})=0$ if and only if equality (1.2) holds for all $\mathbf{g} \in C_{c}^{1}\left(\mathbb{R}^{n}, \mathbb{R}^{n}\right)$. As a matter of fact, in our setting of $W^{1, p}$ maps with $p>n-1$, condition $\mathcal{E}(\mathbf{u})=0$ also corresponds to saying that the graph of $\mathbf{u}$ is a current with no boundary in $\Omega \times \mathbb{R}^{n}$ (see $[38,39,16]$ ).

One of the main results of the paper is a characterization of a class of functions that are orientation preserving and do not exhibit cavitation. As said before, the example of [44] shows that conditions $\mathcal{E}(\mathbf{u})=0$ and $\operatorname{Det} D \mathbf{u}=\operatorname{det} D \mathbf{u}$ are not equivalent, nor does condition $\operatorname{det} D \mathbf{u}>0$ a.e. $\operatorname{imply} \operatorname{deg}(\mathbf{u}, U, \cdot) \geq 0$. Nevertheless, when they are put together, and under some complementary assumptions, they are actually equivalent.

Theorem 1.1. Let $p>n-1$ and suppose that $\mathbf{u} \in W^{1, p}\left(\Omega, \mathbb{R}^{n}\right)$ satisfies $\operatorname{det} D \mathbf{u} \in L_{\text {loc }}^{1}(\Omega)$. The following conditions are equivalent:

a) $\mathcal{E}(\mathbf{u})=0$ and $\operatorname{det} D \mathbf{u}>0$ a.e.

b) $(\operatorname{adj} D \mathbf{u}) \mathbf{u} \in L_{\text {loc }}^{1}\left(\Omega, \mathbb{R}^{n}\right), \operatorname{det} D \mathbf{u}(\mathbf{x}) \neq 0$ for a.e. $\mathbf{x} \in \Omega$, $\operatorname{Det} D \mathbf{u}=\operatorname{det} D \mathbf{u}$, and $\operatorname{deg}(\mathbf{u}, B, \cdot) \geq 0$ for all balls $B$ for which $\operatorname{deg}(\mathbf{u}, B, \cdot)$ is defined.

Throughout this paper, $p>n-1$ is fixed (the borderline case $p=n-1$ is yet to be explored, see [28] for a partial result). We call $\mathcal{A}_{p}$ the class of functions satisfying the conditions of Theorem 1.1. As a particular case of the theory of $[42,44]$ (see also the earlier approaches $[59,38]$ ), it turns out that $\mathcal{A}_{p}$ is a suitable class for proving existence of minimizers of polyconvex functionals in nonlinear elasticity. To have an idea of how big the set $\mathcal{A}_{p}$ is, we point out that, as a consequence of [63], if $\mathbf{u} \in W^{1, p}\left(\Omega, \mathbb{R}^{n}\right)$ satisfies $\operatorname{cof} D \mathbf{u} \in L^{\frac{n}{n-1}}\left(\Omega, \mathbb{R}^{n \times n}\right)$ and $\operatorname{det} D \mathbf{u}>0$ a.e. then $\mathbf{u} \in \mathcal{A}_{p}$. We also show that maps in $\mathcal{A}_{p}$ satisfy a monotonicity property closely related to Manfredi's [56] notion (see also [41] and [47, Ch. 2]). As a consequence, functions in $\mathcal{A}_{p}$ admit a representative that is continuous except for a set of $\mathcal{H}^{n-p}$-measure zero and is differentiable a.e.

The second main theorem of the paper is a local invertibility result for functions in $\mathcal{A}_{p}$ : given $\mathbf{u} \in \mathcal{A}_{p}$, for a.e. $\mathbf{x} \in \Omega$ there exists $r_{\mathbf{x}}>0$ such that the ball $B:=B\left(\mathbf{x}, r_{\mathbf{x}}\right)$ satisfies

$$
\left.\mathbf{u}\right|_{B} \text { satisfies INV, } \mathbf{u}(B)=\operatorname{im}_{\mathrm{T}}(\mathbf{u}, B) \text { a.e. and }\left(\left.\mathbf{u}\right|_{B}\right)^{-1} \in W^{1,1}\left(\operatorname{im}_{\mathrm{T}}(\mathbf{u}, B), \mathbb{R}^{n}\right) .
$$

The set $\operatorname{im}_{\mathrm{T}}(\mathbf{u}, B)$ is the topological image of $B$ under $\mathbf{u}$ (see Definition 3.4; the concept is due to Šverák [70]), and $\mathbf{u}(B)$ is, in truth, the image of a suitable subset of $B$ of full measure under $\mathbf{u}$ (see Definition 2.11; the concept is due to Müller \& Spector [61]). Thanks to the continuity of the degree, $\operatorname{im}_{\mathrm{T}}(\mathbf{u}, B)$ is open. Hence property (1.3) shows that $\mathbf{u}$ is an open map, up to sets of measure zero. This is important in order to give a rigorous definition of the set $\mathbf{u}(\Omega)$ appearing in the models with varying domains that we have in mind; in $W^{1, p}$ with $p \leq n$, this is a delicate matter since two functions $\mathbf{u}$ and $\mathbf{u}_{1}$ that coincide a.e. might have images $\mathbf{u}(\Omega)$ and $\mathbf{u}_{1}(\Omega)$ that differ in a set of positive measure (see, e.g., [61] or [47, Ch. 4]). Thus, property (1.3) expresses that the class $\mathcal{A}_{p}$ seems to be the counterpart of the concept of an orientation-preserving local homeomorphism in $W^{1, p}$. This local invertibility result shows, in particular, that the pathological behaviour of one-to-one a.e. maps with det $D \mathbf{u}>0$ not satisfying INV (see [61, Sect. 11], [62, Sect. 5] and [44, Sect. 7] for some examples) is excluded when cavitation is prohibited.

Moreover, if a sequence $\left\{\mathbf{u}_{j}\right\}_{j \in \mathbb{N}} \subset \mathcal{A}_{p}$ tends to $\mathbf{u} \in \mathcal{A}_{p}$ weakly in $W^{1, p}$, conditions (1.3) are satisfied simultaneously for all members $\mathbf{u}_{j}$ of a subsequence. Furthermore, the local inverses $\left(\left.\mathbf{u}_{j}\right|_{B}\right)^{-1}$ converge to $\left(\left.\mathbf{u}\right|_{B}\right)^{-1}$, as well as their gradients, adjoints and determinants. The convergence is in the appropriate topology to make the direct method of the calculus of variations work for $\left(\left.\mathbf{u}\right|_{B}\right)^{-1}$. This local invertibility 
result generalizes the work of Fonseca \& Gangbo [32] from $p=n$ to $p>n-1$. In the context of geometric function theory and quasiregular mappings, the existence of local homeomorphisms has been studied by $[52,51]$, again for the exponent $p=n$.

This theorem on invertibility and stability of the inverse is especially suitable to prove lower semicontinuity for functionals of the form

$$
\int_{\mathbf{u}(\Omega)} W(D \mathbf{w}(\mathbf{y})) \mathrm{d} \mathbf{y}
$$

and

$$
\int_{\Omega} W_{0}(D \mathbf{u}(\mathbf{x}), \mathbf{w}(\mathbf{u}(\mathbf{x}))) \mathrm{d} \mathbf{x} .
$$

The difficulty of the analysis of (1.4) relies in the fact that the domain $\mathbf{u}(\Omega)$ of $\mathbf{w}$ varies along a minimizing sequence $\left\{\mathbf{u}_{j}\right\}_{j \in \mathbb{N}}$. This obstacle is overcome by working in subdomains $V \subset \subset \mathbf{u}(\Omega)$ such that $V \subset \mathbf{u}_{j}(\Omega)$ for all $j$. On the other hand, the key difficulty of (1.5) is the composition $\mathbf{w} \circ \mathbf{u}$, since, in general, it is not continuous with respect to the weak topology of the corresponding functional spaces. We solve that by working in balls $B \subset \Omega$ where $\mathbf{u}$ and all $\mathbf{u}_{j}$ are invertible and perform a change of variables that brings the analysis of the energy to the deformed configuration, again using the local invertibility property (1.3).

Thus, the results are suitable to show existence of minimizers for variational models that involve reference and deformed configurations simultaneously. We have chosen two examples: nematic elastomers and magnetoelasticity. In this introduction we only explain a particular case of the former.

Recently, in [11], a model for nematic elastomers was investigated, where the elastic behaviour of the polymer chains is coupled to the orientational order of the nematic mesogens through the minimization a total free energy

$$
I(\mathbf{u}, \mathbf{n})=\int_{\Omega} W_{\mathrm{mec}}(D \mathbf{u}(\mathbf{x}), \mathbf{n}(\mathbf{u}(\mathbf{x}))) \mathrm{d} \mathbf{x}+\int_{\mathbf{u}(\Omega)}|D \mathbf{n}(\mathbf{y})|^{2} \mathrm{~d} \mathbf{y}
$$

that consists of a polyconvex energy term depending on the deformation $\mathbf{u}$ (defined on the reference configuration $\Omega$ ), and of a Frank energy term penalizing spatial variations of the nematic director $\mathbf{n}$ (defined in the deformed configuration $\mathbf{u}(\Omega)$ ). Thus, this model combines (1.4) and (1.5). We refer the reader to $[11,26,74]$ and the references therein (see also Subsection 8.1) for its physical motivation. This allows us to reduce the exponent $p=n$ in the coercivity assumption of [11] to $p>n-1$. We also establish the existence of minimizers for the Landau-de Gennes model for nematic elastomers of [14] and one in magnetoelasticity studied in $[69,53]$, under more realistic hypotheses than in those papers.

The outline of the paper is as follows. In Section 2 we set the notation and recall some previous results used in the proof of our theorems. In Section 3 we explain the degree for $W^{1, p}$ maps. We also recall Šverák's [70] notion of topological image and Müller \& Spector's [61] condition INV. In Section 4 we prove Theorem 1.1. In Section 5 we analyze fine properties of functions in $\mathcal{A}_{p}$, notably, weak monotonicity and its consequences, and the Sobolev regularity of the local inverse stated in (1.3). In Section 6 we prove that property (1.3) is stable under weak convergence in $W^{1, p}$. In Section 7 we establish lower semicontinuity results for integrals of the form (1.4) and (1.5), and also for Div-quasiconvex integrands under incompressibility. Finally, in Section 8 we apply the above analysis to show the well-posedness of variational models for nematic elastomers and magnetoelasticity.

\section{Notation and preliminary results}

In this section we set the notation and concepts of the paper, and state some preliminary results. Part of those results are standard in the theory of weakly differentiable functions (see, e.g., [30, 29, 2]), and part are collected from the works by $[61,16,42,43,44,46]$ on cavitation. 


\subsection{General notation}

We will work in dimension $n \geq 2$, and $\Omega$ is a bounded open set of $\mathbb{R}^{n}$. Vector-valued and matrix-valued quantities will be written in boldface. Coordinates in the reference configuration will be denoted by $\mathbf{x}$, and in the deformed configuration by $\mathbf{y}$.

The closure of a set $A$ is denoted by $\bar{A}$, its boundary by $\partial A$, and its characteristic function by $\chi_{A}$. Given two sets $U, V$ of $\mathbb{R}^{n}$, we will write $U \subset \subset V$ if $U$ is bounded and $\bar{U} \subset V$. The open ball of radius $r>0$ centred at $\mathbf{x} \in \mathbb{R}^{n}$ is denoted by $B(\mathbf{x}, r)$; unless otherwise stated, a ball is understood to be open. The function dist indicates the distance from a point to a set, or between two sets.

Given a square matrix $\mathbf{A} \in \mathbb{R}^{n \times n}$, its determinant is denoted by $\operatorname{det} \mathbf{A}$. The adjugate matrix adj $\mathbf{A}$ satisfies $(\operatorname{det} \mathbf{A}) \mathbf{I}=\mathbf{A}$ adj $\mathbf{A}$, where $\mathbf{I}$ denotes the identity matrix. The transpose of adj $\mathbf{A}$ is the cofactor cof $\mathbf{A}$. If $\mathbf{A}$ is invertible, its inverse is denoted by $\mathbf{A}^{-1}$. The inner (dot) product of vectors and of matrices will be denoted by $\cdot$. The Euclidean norm of a vector $\mathbf{x}$ is denoted by $|\mathbf{x}|$, and the associated matrix norm is also denoted by $|\cdot|$. Given $\mathbf{a}, \mathbf{b} \in \mathbb{R}^{n}$, the tensor product $\mathbf{a} \otimes \mathbf{b}$ is the $n \times n$ matrix whose component $(i, j)$ is $a_{i} b_{j}$. The set $\mathbb{R}_{+}^{n \times n}$ denotes the subset of matrices in $\mathbb{R}^{n \times n}$ with positive determinant. The set $\mathbb{S}^{n-1}$ denotes the subset of unit vectors in $\mathbb{R}^{n}$.

The Lebesgue measure in $\mathbb{R}^{n}$ is denoted by $\mathcal{L}^{n}$, and the $s$-dimensional Hausdorff measure by $\mathcal{H}^{s}$ for real $s \geq 0$; in most cases we will deal with $\mathcal{H}^{n-1}$. The abbreviation a.e. stands for almost everywhere or almost every; unless otherwise stated, it refers to the Lebesgue $\mathcal{L}^{n}$ measure. For $1 \leq p \leq \infty$, the Lebesgue $L^{p}$, Sobolev $W^{1, p}$ and bounded variation $B V$ spaces are defined in the usual way. So are the functions of class $C^{k}$, for $k$ a positive integer of infinity, and their versions $C_{c}^{k}$ of compact support. The set of (positive or vector-valued) Radon measures is denoted by $\mathcal{M}$. The conjugate exponent of $p$ is written $p^{\prime}$. We do not identify functions that coincide a.e.; moreover an $L^{p}$ or $W^{1, p}$ function may eventually be defined only at a.e. point of its domain. We will indicate the domain and target space, as in, for example, $L^{p}\left(\Omega, \mathbb{R}^{n}\right)$, except if the target space is $\mathbb{R}$, in which case we will simply write $L^{p}(\Omega)$; the corresponding norm is written $\|\cdot\|_{L^{p}\left(\Omega, \mathbb{R}^{n}\right)}$. Given $S \subset \mathbb{R}^{n}$, the space $L^{p}(\Omega, S)$ denotes the set of $\mathbf{u} \in L^{p}\left(\Omega, \mathbb{R}^{n}\right)$ such that $\mathbf{u}(\mathbf{x}) \in S$ for a.e. $\mathbf{x} \in \Omega$, and analogously for other function spaces. The space $W_{\text {loc }}^{1, p}(\Omega)$ is the set of funcions $\mathbf{u}$ defined in $\Omega$ such that $\left.\mathbf{u}\right|_{A} \in W^{1, p}(A)$ for any open $A \subset \subset \Omega$; we will analogously use the subscript loc for other function spaces. Weak convergence (typically, in $L^{p}$ or $W^{1, p}$ ) is indicated by $\rightarrow$, while $\stackrel{*}{\rightarrow}$ is the symbol for weak*

convergence in $\mathcal{M}$ or in $B V$. The supremum norm in a set $A$ (typically, a sphere) is indicated by $\|\cdot\|_{\infty, A}$, while $f_{A}$ denotes the integral in $A$ divided by the measure of $A$. The identity function in $\mathbb{R}^{n}$ is denoted by id. The support of a function is indicated by spt.

The distributional derivative of a Sobolev function $\mathbf{u}$ is written $D \mathbf{u}$, which is defined a.e. If $\mathbf{u}$ is differentiable at $\mathbf{x}$, its derivative is denoted by $D \mathbf{u}(\mathbf{x})$, while if $\mathbf{u}$ is differentiable everywhere, the derivative function is also denoted by $D \mathbf{u}$. Other notions of differentiability, which carry different notations, are explained in Section 2.2 below.

If $\mu$ is a measure on a set $U$, and $V$ is a $\mu$-measurable subset of $U$, then the restriction of $\mu$ to $V$ is denoted by $\mu\llcorner V$. The measure $|\mu|$ denotes the total variation of $\mu$.

Given two sets $A, B$ of $\mathbb{R}^{n}$, we write $A \subset B$ a.e. if $\mathcal{L}^{n}(A \backslash B)=0$, while $A=B$ a.e. means $A \subset B$ a.e. and $B \subset A$ a.e. An analogous meaning is given to the expression $\mathcal{H}^{n-1}$-a.e. With $\triangle$ we denote the symmetric difference of sets: $A \triangle B:=(A \backslash B) \cup(B \backslash A)$.

In the proofs of convergence, we will continuously use subsequences, which not be relabelled.

\subsection{Approximate continuity and approximate differentiability}

The density $D(A, \mathbf{x})$ and upper density $\bar{D}(A, \mathbf{x})$ of a measurable set $A \subset \mathbb{R}^{n}$ at an $\mathbf{x} \in \mathbb{R}^{n}$ are defined, respectively, as

$$
D(A, \mathbf{x}):=\lim _{r \searrow 0} \frac{\mathcal{L}^{n}(A \cap B(\mathbf{x}, r))}{\mathcal{L}^{n}(B(\mathbf{x}, r))}, \quad \bar{D}(A, \mathbf{x}):=\limsup _{r \searrow 0} \frac{\mathcal{L}^{n}(A \cap B(\mathbf{x}, r))}{\mathcal{L}^{n}(B(\mathbf{x}, r))} .
$$

The following notions are due to Federer [30] (see also [61, Def. 2.3] or [2, Def. 4.31]). 
Definition 2.1. Let $\mathbf{u}: \Omega \rightarrow \mathbb{R}^{n}$ be a measurable function, and consider $\mathbf{x}_{0} \in \Omega$.

a) We say that the approximate limit of $\mathbf{u}$ at $\mathbf{x}_{0}$ is $\mathbf{y}_{0}$ when

$$
D\left(\left\{\mathbf{x} \in \Omega:\left|\mathbf{u}(\mathbf{x})-\mathbf{y}_{0}\right| \geq \delta\right\}, \mathbf{x}_{0}\right)=0 \text { for each } \delta>0 .
$$

In this case, we write ap $\lim _{\mathbf{x} \rightarrow \mathbf{x}_{0}} \mathbf{u}(\mathbf{x})=\mathbf{y}_{0}$. We say that $\mathbf{u}$ is approximately continuous at $\mathbf{x}_{0}$ if $\mathbf{u}$ is defined at $\mathbf{x}_{0}$ and ap $\lim _{\mathbf{x} \rightarrow \mathbf{x}_{0}} \mathbf{u}(\mathbf{x})=\mathbf{u}\left(\mathbf{x}_{0}\right)$.

b) We say that $\mathbf{u}$ is approximately differentiable at $\mathbf{x}_{0}$ if $\mathbf{u}$ is approximately continuous at $\mathbf{x}_{0}$ and there exists $\mathbf{F} \in \mathbb{R}^{n \times n}$ such that

$$
D\left(\left\{\mathbf{x} \in \Omega \backslash\left\{\mathbf{x}_{0}\right\}: \frac{\left|\mathbf{u}(\mathbf{x})-\mathbf{u}\left(\mathbf{x}_{0}\right)-\mathbf{F}\left(\mathbf{x}-\mathbf{x}_{0}\right)\right|}{\left|\mathbf{x}-\mathbf{x}_{0}\right|} \geq \delta\right\}, \mathbf{x}_{0}\right)=0 \text { for each } \delta>0 .
$$

In this case, $\mathbf{F}$ is uniquely determined, called the approximate differential of $\mathbf{u}$ at $\mathbf{x}_{0}$, and denoted by $\nabla \mathbf{u}\left(\mathbf{x}_{0}\right)$.

c) We denote the set of approximate differentiability points of $\mathbf{u}$ by $\Omega_{d}$, or, when we want to emphasize the dependence on $\mathbf{u}$, by $\Omega_{\mathbf{u}, d}$.

We shall use the fact that maps in $W^{1, p}\left(\Omega, \mathbb{R}^{n}\right)$ are approximately continuous ouside a set of zero $p$ capacity, denoted by cap ${ }_{p}$. We follow here the statement of [61, Prop. 2.8]; for a thorough discussion of precise representatives and capacities, we refer to [75, 29].

Proposition 2.2. Let $1 \leq p<n$ and $\mathbf{u} \in W^{1, p}\left(\Omega, \mathbb{R}^{n}\right)$. Let $p^{*}:=n p /(n-p)$ be the Sobolev conjugate exponent. Denote by $P$ the set of points $\mathbf{x}_{0} \in \Omega$ where the following property fails: there exists $\mathbf{u}^{*}\left(\mathbf{x}_{0}\right) \in \mathbb{R}^{n}$ such that

$$
\lim _{r \searrow 0} f_{B\left(\mathbf{x}_{0}, r\right)}\left|\mathbf{u}(\mathbf{x})-\mathbf{u}^{*}\left(\mathbf{x}_{0}\right)\right|^{p^{*}} \mathrm{~d} \mathbf{x}=0 .
$$

Then $\operatorname{cap}_{p}(P)=0$.

Remark 2.3. a) By Jensen's inequality,

$$
\mathbf{u}^{*}\left(\mathbf{x}_{0}\right)=\lim _{r \searrow 0} f_{B\left(\mathbf{x}_{0}, r\right)} \mathbf{u}(\mathbf{x}) \mathrm{d} \mathbf{x}
$$

for every $\mathbf{x}_{0} \in \Omega \backslash P$. Moreover, $\mathbf{u}(\mathbf{x})=\mathbf{u}^{*}(\mathbf{x})$ for a.e. $\mathbf{x} \in \Omega$ by Lebesgue's differentiation theorem.

b) It is well known that limit (2.1) implies that ap $\lim _{\mathbf{x} \rightarrow \mathbf{x}_{0}} \mathbf{u}(\mathbf{x})=\mathbf{u}^{*}\left(\mathbf{x}_{0}\right)$ and that if $\mathbf{u} \in L_{\mathrm{loc}}^{\infty}\left(\Omega, \mathbb{R}^{n}\right)$ then the converse also holds (a proof can be found, e.g., in [2, Prop. 3.65]).

c) The property $\operatorname{cap}_{p}(P)=0$ implies that $\mathcal{H}^{\delta}(P)=0$ for every $\delta>n-p$. In particular, if $p>n-1$ then $\mathcal{H}^{1}(P)=0$, so for each $\mathbf{x} \in \Omega$ there exists an $\mathcal{L}^{1}$-null set $N \subset(0, \infty)$ such that $P \cap \partial B(\mathbf{x}, r)=\varnothing$ for all $r \in(0, \operatorname{dist}(\mathbf{x}, \partial \Omega)) \backslash N$.

d) If $p>n$ then, by Morrey's embedding, $\mathbf{u}$ has a representative $\mathbf{u}^{*}$ that is continuous; consequently, limit (2.1) holds for every $\mathbf{x}_{0} \in \Omega$ and every $p^{*}<\infty$. If $p=n$, since in this paper we will work under the assumption $\operatorname{det} D \mathbf{u}>0$ a.e., we have again that $\mathbf{u}$ has a continuous representative $\mathbf{u}^{*}$ (see, e.g., [73, Th. 2.3.2] or [70, Th. 5]). In either of these cases, when invoking Proposition 2.2 we will tacitly understand that $P=\varnothing$.

By the Calderón-Zygmund theorem (see, e.g., [2, Th. 3.83]), $B V$ functions are approximately differentiable a.e. We point out the following remark on the approximate differentiability of two different representatives. 
Remark 2.4. For maps $\mathbf{u}_{1}, \mathbf{u}_{2} \in W^{1, p}\left(\Omega, \mathbb{R}^{n}\right)$ that coincide a.e., the sets $\Omega_{\mathbf{u}_{1}, d}$ and $\Omega_{\mathbf{u}_{2}, d}$ may not be the same, but $\mathbf{u}_{1}(\mathbf{x})=\mathbf{u}_{2}(\mathbf{x})$ and $\nabla \mathbf{u}_{1}(\mathbf{x})=\nabla \mathbf{u}_{2}(\mathbf{x})$ for all $\mathbf{x} \in \Omega_{\mathbf{u}_{1}, d} \cap \Omega_{\mathbf{u}_{2}, d}$ (which is a set of full measure). Indeed, if $\mathbf{x}_{0} \in \Omega_{\mathbf{u}_{i}, d}(i=1,2)$ then $\mathbf{u}_{i}\left(\mathbf{x}_{0}\right)=$ ap $\lim _{\mathbf{x} \rightarrow \mathbf{x}_{0}} \mathbf{u}_{i}(\mathbf{x})$, but two functions that coincide a.e. have the same approximate limit. Similarly, if $\mathbf{x}_{0} \in \Omega_{\mathbf{u}_{1}, d}$ and $\mathbf{u}_{1}\left(\mathbf{x}_{0}\right)=\mathbf{u}_{2}\left(\mathbf{x}_{0}\right)$ then $\mathbf{x}_{0} \in \Omega_{\mathbf{u}_{2}, d}$ and $\nabla \mathbf{u}_{1}\left(\mathbf{x}_{0}\right)=\nabla \mathbf{u}_{2}\left(\mathbf{x}_{0}\right)$.

Definition 2.5. Let $\mathbf{u}: \Omega \rightarrow \mathbb{R}^{n}$ be a measurable function, and consider $\mathbf{x}_{0} \in \Omega$. We say that $\mathbf{u}$ has a regular approximate differential $\mathbf{F}$ at $\mathbf{x}_{0}$ when $\mathbf{u}$ is approximately continuous at $\mathbf{x}_{0}$ and there exists a measurable set $A \subset(0, \infty)$ satisfying $D(A, 0)=1 / 2$ such that for every $r \in A$ the map $\mathbf{u}$ is well defined at every point of $\partial B\left(\mathbf{x}_{0}, r\right)$ and

$$
\lim _{\substack{r \rightarrow 0 \\ r \in A}} \sup _{\mathbf{x} \in \partial B\left(\mathbf{x}_{0}, r\right)} \frac{\left|\mathbf{u}(\mathbf{x})-\mathbf{u}\left(\mathbf{x}_{0}\right)-\mathbf{F}\left(\mathbf{x}-\mathbf{x}_{0}\right)\right|}{\left|\mathbf{x}-\mathbf{x}_{0}\right|}=0 .
$$

In this case, $\mathbf{x}_{0} \in \Omega_{d}$ and $\mathbf{F}=\nabla \mathbf{u}\left(\mathbf{x}_{0}\right)$.

Definition 2.5 is due to Goffman \& Ziemer [40, Sect. 3] (see also [61, Def. 8.2], [32, Def. 2.6] or [31, Def. 5.2], although the name of this notion slightly differs), who proved in [40, Th. 3.4] (see also [61, Prop. 8.3] or [31, Th. 5.21]) that $W^{1, p}$ maps with $p>n-1$ have a representative with a regular approximate differential a.e.

Proposition 2.6. Let $\mathbf{u} \in W^{1, p}\left(\Omega, \mathbb{R}^{n}\right)$ with $p>n-1$. Let $\mathbf{u}^{*}$ and $P$ be as in Proposition 2.2. Then $\mathbf{u}^{*}: \Omega \backslash P \rightarrow \mathbb{R}^{n}$ has a regular approximate differential at a.e. point of $\Omega$, and $\nabla \mathbf{u}^{*}$ coincides a.e. with the distributional derivative Du.

\subsection{Area formulas and geometric image}

We recall that a function $\mathbf{u}: \Omega \rightarrow \mathbb{R}^{n}$ defined everywhere satisfies Lusin's $N$ condition if the image of a subset of $\Omega$ of measure zero is a set of measure zero. It satisfies Lusin's $N^{-1}$ condition if the preimage of a subset of $\mathbb{R}^{n}$ of measure zero is a set of measure zero.

Given a measurable $\mathbf{u}: \Omega \rightarrow \mathbb{R}^{n}$ that is approximately differentiable a.e., for any $A \subset \mathbb{R}^{n}$ and $\mathbf{y} \in \mathbb{R}^{n}$, we denote by $\mathcal{N}_{A}(\mathbf{y})$ the number of $\mathbf{x} \in \Omega_{d} \cap A$ such that $\mathbf{u}(\mathbf{x})=\mathbf{y}$. We will use the following version of Federer's [30] area formula, the formulation of which is taken from [61, Prop. 2.6].

Proposition 2.7. Let $\mathbf{u}: \Omega \rightarrow \mathbb{R}^{n}$ be measurable and approximately differentiable a.e. Then, for any measurable set $A \subset \Omega$ and any measurable function $\varphi: \mathbb{R}^{n} \rightarrow \mathbb{R}$,

$$
\int_{A} \varphi(\mathbf{u}(\mathbf{x}))|\operatorname{det} D \mathbf{u}(\mathbf{x})| \mathrm{d} \mathbf{x}=\int_{\mathbb{R}^{n}} \varphi(\mathbf{y}) \mathcal{N}_{A}(\mathbf{y}) \mathrm{d} \mathbf{y},
$$

whenever either integral exists. Moreover, given $\psi: A \rightarrow \mathbb{R}$ measurable, the function $\bar{\psi}: \mathbf{u}\left(\Omega_{d} \cap A\right) \rightarrow \mathbb{R}$ defined by

$$
\bar{\psi}(\mathbf{y}):=\sum_{\substack{\mathbf{x} \in \Omega_{d} \cap A \\ \mathbf{u}(\mathbf{x})=\mathbf{y}}} \psi(\mathbf{x})
$$

is measurable and satisfies

$$
\int_{A} \psi(\mathbf{x}) \varphi(\mathbf{u}(\mathbf{x}))|\operatorname{det} D \mathbf{u}(\mathbf{x})| \mathrm{d} \mathbf{x}=\int_{\mathbf{u}\left(\Omega_{d} \cap A\right)} \bar{\psi}(\mathbf{y}) \varphi(\mathbf{y}) \mathrm{d} \mathbf{y},
$$

whenever the integral of the left-hand side exists.

The following consequences of Proposition 2.7 hold.

Lemma 2.8. Let $\mathbf{u}: \Omega \rightarrow \mathbb{R}^{n}$ be measurable and approximately differentiable a.e.

a) If $E \subset \Omega$ has measure zero then $\mathcal{N}_{E}=0$ a.e. and $\mathbf{u}\left(\Omega_{d} \cap E\right)$ has measure zero. 
b) If $A=\bigcup_{k \in \mathbb{N}} A_{k}$ a.e. for a disjoint family $\left\{A_{k}\right\}_{k \in \mathbb{N}}$, then

$$
\mathcal{N}_{A}=\sum_{k \in \mathbb{N}} \mathcal{N}_{A_{k}} \quad \text { a.e. }
$$

c) If $\operatorname{det} D \mathbf{u}(\mathbf{x}) \neq 0$ for a.e. $\mathbf{x} \in \Omega$, then $\mathbf{u}$ satisfies Lusin's $N^{-1}$ condition.

Proof. Property a) is an immediate consequence of Proposition 2.7. For b), we notice that

$$
\mathcal{N}_{\bigcup_{k \in \mathbb{N}} A_{k}}=\sum_{k \in \mathbb{N}} \mathcal{N}_{A_{k}}
$$

and apply a) to $A \triangle \bigcup_{k \in \mathbb{N}} A_{k}$.

To prove $c$ ), let $A \subset \mathbf{u}^{-1}(B)$ be measurable, and call $\Omega_{1}$ the set of $\mathbf{x} \in \Omega_{d}$ such that $\operatorname{det} D \mathbf{u}(\mathbf{x}) \neq 0$. Then, thanks to Proposition 2.7 we have

$$
\mathcal{L}^{n}(A)=\int_{\Omega_{1} \cap A} \mathrm{~d} \mathbf{x}=\int_{\mathbf{u}\left(\Omega_{1} \cap A\right)} \sum_{\substack{\mathbf{x} \in \Omega_{1} \cap A \\ \mathbf{u}(\mathbf{x})=\mathbf{y}}} \frac{1}{|\operatorname{det} D \mathbf{u}(\mathbf{x})|} \mathrm{d} \mathbf{y} \leq \int_{B} \sum_{\substack{\mathbf{x} \in \Omega_{1} \cap A \\ \mathbf{u}(\mathbf{x})=\mathbf{y}}} \frac{1}{|\operatorname{det} D \mathbf{u}(\mathbf{x})|} \mathrm{d} \mathbf{y}=0 .
$$

Thus, $\mathcal{L}^{n}(A)=0$ and, consequently, $\mathbf{u}^{-1}(B)$ is measurable with $\mathcal{L}^{n}\left(\mathbf{u}^{-1}(B)\right)=0$.

As a consequence of Lemma 2.8 a), $\left.\mathbf{u}\right|_{\Omega_{0}}$ satisfies Lusin's $N$ condition and the formulas of Proposition 2.7 still hold if $\Omega_{d}$ is replaced by any $\Omega^{\prime} \subset \Omega_{d}$ with $\mathcal{L}^{n}\left(\Omega^{\prime}\right)=\mathcal{L}^{n}(\Omega)$. The conclusion of Lemma $2.8 c$ ) will also be used several times and, in particular, is important because of the following result, which is well known and has an easy proof (see, e.g., [4, p. 73]).

Lemma 2.9. Let $\mathbf{u}: \Omega \rightarrow \mathbb{R}^{n}$ be measurable satisfying Lusin's $N^{-1}$ condition. Let $f: \mathbb{R}^{n} \rightarrow \mathbb{R}$ be measurable. Then $f \circ \mathbf{u}$ is measurable.

We recall the definition of a.e. invertibility and mention some basic properties that are relevant for the analysis of local invertibility.

Definition 2.10. A function $\mathbf{u}: \Omega \rightarrow \mathbb{R}^{n}$ is said to be one-to-one a.e. in a subset $A$ of $\Omega$ if there exists an $\mathcal{L}^{n}$-null subset $N$ of $A$ such that $\left.\mathbf{u}\right|_{A \backslash N}$ is one-to-one.

Thanks to Lemma 2.8 , an a.e. approximately differentiable map $\mathbf{u}: \Omega \rightarrow \mathbb{R}^{n}$ such that $\operatorname{det} \nabla \mathbf{u} \neq 0$ a.e. is one-to-one a.e. in a measurable set $A \subset \Omega$ if and only if $\mathcal{N}_{A} \leq 1$ a.e. in $\mathbb{R}^{n}$. Of course, if $B \subset A$ and $\mathbf{u}$ is one-to-one a.e. in $A$ then so is in $B$.

Now we present the notion of the geometric image of a set (see [61, 16, 44]).

Definition 2.11. Let $\mathbf{u} \in W^{1, p}\left(\Omega, \mathbb{R}^{n}\right)$ and suppose that $\operatorname{det} D \mathbf{u}(\mathbf{x}) \neq 0$ for a.e. $\mathbf{x} \in \Omega$. Define $\Omega_{0}$ as the set of $\mathbf{x} \in \Omega$ for which the following are satisfied:

a) $\mathbf{u}$ is approximately differentiable at $\mathbf{x}$ and $\operatorname{det} \nabla \mathbf{u}(\mathbf{x}) \neq 0$; and

b) there exist $\mathbf{w} \in C^{1}\left(\mathbb{R}^{n}, \mathbb{R}^{n}\right)$ and a compact set $K \subset \Omega$ of density 1 at $\mathbf{x}$ such that $\left.\mathbf{u}\right|_{K}=\left.\mathbf{w}\right|_{K}$ and $\left.\nabla \mathbf{u}\right|_{K}=\left.D \mathbf{w}\right|_{K}$

In order to emphasise the dependence on $\mathbf{u}$, the notation $\Omega_{\mathbf{u}, 0}$ will also be employed. For any measurable set $A$ of $\Omega$, we define the geometric image of $A$ under $\mathbf{u}$ as $\mathbf{u}\left(A \cap \Omega_{0}\right)$, and denote it by $\operatorname{im}_{\mathrm{G}}(\mathbf{u}, A)$.

The set $\Omega_{0}$ is of full measure in $\Omega$. Indeed, the Calderón-Zygmund theorem shows that property $a$ ) is satisfied a.e., while standard arguments, essentially due to Federer [30, Thms. 3.1.8 and 3.1.16] (see also [61, Prop. 2.4] and [16, Rk. 2.5]), show that property $b$ ) is also satisfied a.e. Note also that $\mathbf{u}$ is well defined at every $\mathbf{x} \in \Omega_{0}$, because of Definition $2.1 \mathrm{~b}$ ).

The reason we consider the set $\Omega_{0}$ of points $\mathbf{x}$ around which $\mathbf{u}$ is almost $C^{1}$ and $\nabla \mathbf{u}(\mathbf{x})$ is invertible is the following measure-theoretic inverse function theorem by Müller \& Spector [61, Lemma 2.5] (see also [43, Lemma 1]). 
Proposition 2.12. Let $\mathbf{u} \in W^{1, p}\left(\Omega, \mathbb{R}^{n}\right)$ satisfy $\operatorname{det} D \mathbf{u}(\mathbf{x}) \neq 0$ for a.e. $\mathbf{x} \in \Omega$. Then, for every $\mathbf{x} \in \Omega_{0}$ and every measurable set $A \subset \Omega$,

$$
D\left(\operatorname{im}_{\mathrm{G}}(\mathbf{u}, A), \mathbf{u}(\mathbf{x})\right)=1 \quad \text { whenever } \quad D(A, \mathbf{x})=1 .
$$

We present the notion of tangential approximate differentiability (cf. [30, Def. 3.2.16]).

Definition 2.13. Let $S \subset \mathbb{R}^{n}$ be a $C^{1}$ differentiable manifold of dimension $n-1$, and let $\mathbf{x}_{0} \in S$. Let $T_{\mathbf{x}_{0}} S$ be the linear tangent space of $S$ at $\mathbf{x}_{0}$. A map $\mathbf{u}: S \rightarrow \mathbb{R}^{n}$ is said to be $\mathcal{H}^{n-1}\llcorner S$-approximately differentiable at $\mathbf{x}_{0}$ if there exists $\mathbf{L} \in \mathbb{R}^{n \times n}$ such that for all $\delta>0$,

$$
\lim _{r \searrow 0} \frac{1}{r^{n-1}} \mathcal{H}^{n-1}\left(\left\{\mathbf{x} \in S \cap B\left(\mathbf{x}_{0}, r\right): \frac{\left|\mathbf{u}(\mathbf{x})-\mathbf{u}\left(\mathbf{x}_{0}\right)-\mathbf{L}\left(\mathbf{x}-\mathbf{x}_{0}\right)\right|}{\left|\mathbf{x}-\mathbf{x}_{0}\right|} \geq \delta\right\}\right)=0 .
$$

In this case, the linear map $\left.\mathbf{L}\right|_{T_{\mathbf{x}_{0}} S}: T_{\mathbf{x}_{0}} S \rightarrow \mathbb{R}^{n}$ is uniquely determined, called the tangential approximate derivative of $\mathbf{u}$ at $\mathbf{x}_{0}$, and is denoted by $\nabla \mathbf{u}\left(\mathbf{x}_{0}\right)$.

We will see in Section 2.4 that the equality $\nabla\left(\left.\mathbf{u}\right|_{S}\right)(\mathbf{x})=\left.\nabla \mathbf{u}(\mathbf{x})\right|_{T_{\mathbf{x}} S}$ holds for most points $\mathbf{x}$ if $\mathbf{u}$ is a Sobolev map.

The following convention will be used throughout the paper.

Convention 2.14. If $\mathbf{u} \in W^{1, p}\left(\Omega, \mathbb{R}^{n}\right)$ and $\left.\mathbf{u}\right|_{\partial U} \in W^{1, p}\left(\partial U, \mathbb{R}^{n}\right)$ for some $C^{1}$ open set $U \subset \subset \Omega$ and some $p>n-1$, then in expressions like $\mathbf{u}(\partial U)$ or $\left.\mathbf{u}\right|_{\partial U}$ we shall be referring to the continuous representative of $\left.\mathbf{u}\right|_{\partial U}$ in $W^{1, p}\left(\partial U, \mathbb{R}^{n}\right)$, which exists thanks to Morrey's embedding theorem. Moreover, we will usually write $\mathbf{u} \in W^{1, p}\left(\partial U, \mathbb{R}^{n}\right)$ instead of $\left.\mathbf{u}\right|_{\partial U} \in W^{1, p}\left(\partial U, \mathbb{R}^{n}\right)$.

Federer's change of variables formula for surface integrals [30, Cor. 3.2.20] (see also [61, Prop. 2.7] and [44, Prop. 2.9]), combined with Lusin's property for Sobolev maps proved by Marcus \& Mizel [57], will play an important role in the paper. We will adopt the following formulation.

Proposition 2.15. Let $\mathbf{u} \in W^{1, p}\left(\Omega, \mathbb{R}^{n}\right), p>n-1$. Suppose that $U$ is a $C^{1}$ open subset of $\Omega$, and $\left.\mathbf{u}\right|_{\partial U} \in W^{1, p}\left(\partial U, \mathbb{R}^{n}\right)$. Assume, further, that $\nabla\left(\left.\mathbf{u}\right|_{\partial U}\right)(\mathbf{x})=\left.\nabla \mathbf{u}(\mathbf{x})\right|_{T_{\mathbf{x}} \partial U}$ for $\mathcal{H}^{n-1}$-a.e. $\mathbf{x} \in \partial U$. Then, for any $\mathcal{H}^{n-1}$-measurable subset $A \subset \partial U$,

$$
\mathcal{H}^{n-1}(\mathbf{u}(A))=\int_{A}|(\operatorname{cof} \nabla \mathbf{u}(\mathbf{x})) \boldsymbol{\nu}(\mathbf{x})| \mathrm{d} \mathcal{H}^{n-1}(\mathbf{x})
$$

where $\boldsymbol{\nu}(\mathbf{x})$ denotes the outward unit normal to $\partial U$ at $\mathbf{x}$.

Remark 2.16. a) By $\mathbf{u}(A)$ we refer to the image of $A$ by the continuous representative of $\left.\mathbf{u}\right|_{\partial U}$ in $W^{1, p}\left(\partial U, \mathbb{R}^{n}\right)$, due to Convention 2.14.

b) We are mostly interested in the facts that $\mathcal{H}^{n-1}(\mathbf{u}(\partial U))<\infty$ and that $\mathcal{H}^{n-1}(\mathbf{u}(A))=0$ for every $\mathcal{H}^{n-1}$-null set $A \subset \partial U$. In particular, $\mathcal{L}^{n}(\mathbf{u}(\partial U))=0$, and $\mathbf{u}(\partial U)=\mathbf{u}\left(\partial U \cap \Omega_{0}\right) \mathcal{H}^{n-1}$-a.e. if $\partial U \subset \Omega_{0}$ $\mathcal{H}^{n-1}$-a.e., where $\Omega_{0}$ is the set of Definition 2.11.

\subsection{A class of good open sets}

In the following definition, given a nonempty open set $U \subset \subset \Omega$ with a $C^{2}$ boundary, we call $d: \Omega \rightarrow \mathbb{R}$ the function given by

$$
d(\mathbf{x}):= \begin{cases}\operatorname{dist}(\mathbf{x}, \partial U) & \text { if } \mathbf{x} \in U \\ 0 & \text { if } \mathbf{x} \in \partial U \\ -\operatorname{dist}(\mathbf{x}, \partial U) & \text { if } \mathbf{x} \in \Omega \backslash \bar{U}\end{cases}
$$

and

$$
U_{t}:=\{\mathbf{x} \in \Omega: d(\mathbf{x})>t\},
$$

for each $t \in \mathbb{R}$. We note (see, e.g., [27, Th. 16.25.2], [70, p. 112] or [61, p. 48]) that there exists $\delta>0$ such that for all $t \in(-\delta, \delta)$, the set $U_{t}$ is open, compactly contained in $\Omega$ and has a $C^{2}$ boundary. 
Definition 2.17. Let $p>n-1$. Let $\mathbf{u} \in W^{1, p}\left(\Omega, \mathbb{R}^{n}\right)$. We define $\mathcal{U}_{\mathbf{u}}$ as the family of nonempty open sets $U \subset \subset \Omega$ with a $C^{2}$ boundary that satisfy the following conditions:

a) $\left.\mathbf{u}\right|_{\partial U} \in W^{1, p}\left(\partial U, \mathbb{R}^{n}\right)$, and $\left.(\operatorname{cof} \nabla \mathbf{u})\right|_{\partial U} \in L^{1}\left(\partial U, \mathbb{R}^{n \times n}\right)$.

b) $\partial U \subset \Omega_{0} \mathcal{H}^{n-1}$-a.e., where $\Omega_{0}$ is the set of Definition 2.11, and $\nabla\left(\left.\mathbf{u}\right|_{\partial U}\right)(\mathbf{x})=\left.\nabla \mathbf{u}(\mathbf{x})\right|_{T_{\mathbf{x}} \partial U}$ for $\mathcal{H}^{n-1}$-a.e. $\mathbf{x} \in \partial U$.

c) $\lim _{\varepsilon \searrow 0} f_{0}^{\varepsilon}\left|\int_{\partial U_{t}}\right| \operatorname{cof} \nabla \mathbf{u}\left|\mathrm{d} \mathcal{H}^{n-1}-\int_{\partial U}\right| \operatorname{cof} \nabla \mathbf{u}\left|\mathrm{d} \mathcal{H}^{n-1}\right| \mathrm{d} t=0$.

d) For every $\mathbf{g} \in C^{1}\left(\mathbb{R}^{n}, \mathbb{R}^{n}\right)$ with $(\operatorname{adj} D \mathbf{u})(\mathbf{g} \circ \mathbf{u}) \in L_{\mathrm{loc}}^{1}\left(\Omega, \mathbb{R}^{n}\right)$,

$$
\lim _{\varepsilon \searrow 0} f_{0}^{\varepsilon}\left|\int_{\partial U_{t}} \mathbf{g}(\mathbf{u}(\mathbf{x})) \cdot\left(\operatorname{cof} \nabla \mathbf{u}(\mathbf{x}) \boldsymbol{\nu}_{t}(\mathbf{x})\right) \mathrm{d} \mathcal{H}^{n-1}(\mathbf{x})-\int_{\partial U} \mathbf{g}(\mathbf{u}(\mathbf{x})) \cdot(\operatorname{cof} \nabla \mathbf{u}(\mathbf{x}) \boldsymbol{\nu}(\mathbf{x})) \mathrm{d} \mathcal{H}^{n-1}(\mathbf{x})\right| \mathrm{d} t=0,
$$

where $\boldsymbol{\nu}_{t}$ denotes the unit outward normal to $U_{t}$ for each $t \in(0, \varepsilon)$, and $\boldsymbol{\nu}$ the unit outward normal to $U$.

Remark 2.18. The fact that $\mathbf{u} \in W^{1, p}\left(\partial U, \mathbb{R}^{n}\right)$ is not enough to ensure that $\left.(\operatorname{cof} \nabla \mathbf{u})\right|_{\partial U} \in L^{1}\left(\partial U, \mathbb{R}^{n \times n}\right)$ because cof $\nabla \mathbf{u}$ involves not only the tangential derivatives of $\mathbf{u}$ but also its normal derivative; recall that $\nabla \mathbf{u}$ is the approximate gradient of $\mathbf{u}: \Omega \rightarrow \mathbb{R}^{n}$ according to Definition $2.1 b$ ).

Remark 2.19. If $\mathbf{u}_{1}, \mathbf{u}_{2} \in W^{1, p}\left(\Omega, \mathbb{R}^{n}\right)$ coincide a.e. and $U \in \mathcal{U}_{\mathbf{u}_{1}} \cap \mathcal{U}_{\mathbf{u}_{2}}$, then $\mathbf{u}_{1}(\mathbf{x})=\mathbf{u}_{2}(\mathbf{x})$ for $\mathcal{H}^{n-1}$ a.e. $\mathbf{x} \in \partial U$. Indeed, both $\Omega_{\mathbf{u}_{1}, 0} \cap \partial U$ and $\Omega_{\mathbf{u}_{2}, 0} \cap \partial U$ are of full $\mathcal{H}^{n-1}$-measure on $\partial U$, and $\mathbf{u}_{1}=\mathbf{u}_{2}$ on $\Omega_{\mathbf{u}_{1}, 0} \cap \Omega_{\mathbf{u}_{2}, 0}$ by Remark 2.4 .

Let $P$ be the singular set of Proposition 2.2. Then, Remark 2.3c), [61, Prop. 2.8] and [43, Lemma 2 and Def. 11] (or [44, Lemma 2.16]) guarantee that there are enough sets in $\mathcal{U}_{\mathbf{u}}$ whose boundaries do not intersect $P$ and which, furthermore, are such that the continuous representative of Convention 2.14 is given by the precise representative $\mathbf{u}^{*}$.

Lemma 2.20. Let $p>n-1$. Let $\mathbf{u} \in W^{1, p}\left(\Omega, \mathbb{R}^{n}\right)$ be such that $\operatorname{det} D \mathbf{u}>0$ a.e. Let $U \subset \subset \Omega$ be a nonempty open set with a $C^{2}$ boundary. Then, for a.e. $t \in(-\delta, \delta)$,

$$
U_{t} \in \mathcal{U}_{\mathbf{u}}, \quad \partial U_{t} \cap P=\varnothing \quad \text { and }\left.\mathbf{u}^{*}\right|_{\partial U_{t}} \text { is continuous. }
$$

Moreover, for each compact $K \subset \Omega$ there exists $U^{\prime} \in \mathcal{U}_{\mathbf{u}}$ such that $K \subset U^{\prime}$.

Remark 2.21. Let $\mathbf{u}^{*}$ be the precise representative of Proposition 2.2. Every set $U \in \mathcal{U}_{\mathbf{u}}$ satisfying $\partial U \cap P=\varnothing$ belongs to $\mathcal{U}_{\mathbf{u}^{*}}$. Indeed, suppose that $U \in \mathcal{U}_{\mathbf{u}}$. Then $\partial U \subset \Omega_{0} \mathcal{H}^{n-1}$-a.e. By definition of $\Omega_{0}, \mathbf{u}$ is approximately differentiable (and, hence, approximately continuous) at every point in $\Omega_{0}$. By Remark $2.3 \mathrm{~b}$ ) and the definition of approximate differentiability, this implies that $\mathbf{u}=\mathbf{u}^{*}$ and $\nabla \mathbf{u}=\nabla \mathbf{u}^{*}$ in $\Omega_{0} \backslash P$. This is enough to see that all the conditions in Definition 2.17 are satisfied.

Lemma 2.22. Let $U_{1}, U_{2} \in \mathcal{U}_{\mathbf{u}}$. If $U_{1} \subset \subset U_{2}$ then $U_{2} \backslash \bar{U}_{1} \in \mathcal{U}_{\mathbf{u}}$. If $\bar{U}_{1} \cap \bar{U}_{2}=\varnothing$ then $U_{1} \cup U_{2} \in \mathcal{U}_{\mathbf{u}}$.

Proof. It is enough to observe that, in the first case, $\partial\left(U_{2} \backslash \bar{U}_{1}\right)=\partial U_{1} \cup \partial U_{2}$ with disjoint union and, in the second case, $\partial\left(U_{1} \cup U_{2}\right)=\partial U_{1} \cup \partial U_{2}$ with disjoint union.

The following result, with slightly different assumptions, was shown in the proof of [42, Th. 2] (see also [43, Th. 2] or [44, Prop. 5.1]).

Proposition 2.23. Let $p>n-1, \mathbf{u} \in W^{1, p}\left(\Omega, \mathbb{R}^{n}\right)$ and $U \in \mathcal{U}_{\mathbf{u}}$. Then there exists a family $\left\{\phi_{\delta}\right\}_{\delta>0}$ in $C_{c}^{1}(\Omega)$ such that $\phi_{\delta} \nearrow \chi_{U}$ pointwise in $\Omega$ as $\delta \searrow 0$ and, for each $\mathbf{g} \in C^{1}\left(\mathbb{R}^{n}, \mathbb{R}^{n}\right)$ with $(\operatorname{adj} D \mathbf{u})(\mathbf{g} \circ \mathbf{u}) \in L_{\text {loc }}^{1}\left(\Omega, \mathbb{R}^{n}\right)$,

$$
\lim _{\delta \searrow 0} \int_{\Omega} \mathbf{g}(\mathbf{u}(\mathbf{x})) \cdot\left(\operatorname{cof} D \mathbf{u}(\mathbf{x}) D \phi_{\delta}(\mathbf{x})\right) \mathrm{d} \mathbf{x}=-\int_{\partial U} \mathbf{g}(\mathbf{u}(\mathbf{x})) \cdot(\operatorname{cof} \nabla \mathbf{u}(\mathbf{x}) \boldsymbol{\nu}(\mathbf{x})) \mathrm{d} \mathcal{H}^{n-1}(\mathbf{x}),
$$

where $\boldsymbol{\nu}$ is the outward normal to $U$. 
The following result is a consequence of Morrey's embedding and Fubini's theorem (see [61, Lemma 2.9] for a proof).

Lemma 2.24. Let $p>n-1$. For each $j \in \mathbb{N}$ let $\mathbf{u}_{j}, \mathbf{u} \in W^{1, p}\left(\Omega, \mathbb{R}^{n}\right)$ satisfy $\mathbf{u}_{j} \rightarrow \mathbf{u}$ in $W^{1, p}\left(\Omega, \mathbb{R}^{n}\right)$ as $j \rightarrow \infty$. Let $U \subset \subset \Omega$ be an open set with a $C^{2}$ boundary. Then there exists $\delta>0$ such that for a.e. $t \in(-\delta, \delta)$,

$$
\mathbf{u}_{j}, \mathbf{u} \in W^{1, p}\left(\partial U_{t}, \mathbb{R}^{n}\right) \quad \text { for all } j \in \mathbb{N}
$$

and, for a subsequence (depending on $t$ ),

$$
\mathbf{u}_{j} \rightarrow \mathbf{u} \quad \text { uniformly on } \partial U_{t} \text { as } j \rightarrow \infty \text {. }
$$

\subsection{Surface energy and distributional determinant}

The following concepts were defined in [42].

Definition 2.25. Let $\mathbf{u}: \Omega \rightarrow \mathbb{R}^{n}$ be measurable and approximately differentiable a.e. Suppose that $\operatorname{det} \nabla \mathbf{u} \in L_{\text {loc }}^{1}(\Omega)$ and $\operatorname{cof} \nabla \mathbf{u} \in L_{\text {loc }}^{1}\left(\Omega, \mathbb{R}^{n \times n}\right)$. For every $\mathbf{f} \in C_{c}^{1}\left(\Omega \times \mathbb{R}^{n}, \mathbb{R}^{n}\right)$, define

$$
\mathcal{E}(\mathbf{u}, \mathbf{f}):=\int_{\Omega}[\operatorname{cof} \nabla \mathbf{u}(\mathbf{x}) \cdot D \mathbf{f}(\mathbf{x}, \mathbf{u}(\mathbf{x}))+\operatorname{det} \nabla \mathbf{u}(\mathbf{x}) \operatorname{div} \mathbf{f}(\mathbf{x}, \mathbf{u}(\mathbf{x}))] \mathrm{d} \mathbf{x}
$$

and

$$
\mathcal{E}(\mathbf{u}):=\sup \left\{\mathcal{E}(\mathbf{u}, \mathbf{f}): \mathbf{f} \in C_{c}^{1}\left(\Omega \times \mathbb{R}^{n}, \mathbb{R}^{n}\right),\|\mathbf{f}\|_{\infty} \leq 1\right\} .
$$

In equation (2.3), Df $\mathbf{f}(\mathbf{x}, \mathbf{y})$ denotes the derivative of $\mathbf{f}(\cdot, \mathbf{y})$ evaluated at $\mathbf{x}$, while $\operatorname{div} \mathbf{f}(\mathbf{x}, \mathbf{y})$ is the divergence of $\mathbf{f}(\mathbf{x}, \cdot)$ evaluated at $\mathbf{y}$.

The functional $\mathcal{E}$ was introduced in [42] to measure the creation of new surface of a deformation. In this paper we are only interested in the case where $\mathbf{u}$ does not create new surface, so $\mathcal{E}(\mathbf{u})=0$. When $\mathbf{u} \in W^{1, p}$, as is the case of interest in this paper, this was shown in [44, Th. 4.6] to be equivalent to the requirement that $\mathbf{u}$ does not exhibit cavitation. Again by [44, Th. 4.6], or just using the density in $C_{c}^{1}\left(\Omega \times \mathbb{R}^{n}, \mathbb{R}^{n}\right)$ of sums of functions of separate variables (see, e.g., [54, Cor. 1.6.5]), one can see that equality $\mathcal{E}(\mathbf{u})=0$ is equivalent to

$$
\int_{\Omega}[\operatorname{cof} \nabla \mathbf{u}(\mathbf{x}) \cdot(\mathbf{g}(\mathbf{u}(\mathbf{x})) \otimes D \phi(\mathbf{x}))+\operatorname{det} \nabla \mathbf{u}(\mathbf{x}) \phi(\mathbf{x}) \operatorname{div} \mathbf{g}(\mathbf{u}(\mathbf{x}))] \mathrm{d} \mathbf{x}=0,
$$

for all $\phi \in C_{c}^{1}(\Omega)$ and $\mathbf{g} \in C_{c}^{1}\left(\mathbb{R}^{n}, \mathbb{R}^{n}\right)$, which, in turn, is equivalent to identities (1.2) in the sense of distributions for all $\mathbf{g} \in C_{c}^{1}\left(\mathbb{R}^{n}, \mathbb{R}^{n}\right)$.

We present the definition of distributional determinant (see [6] or [60]). With $\langle\cdot, \cdot\rangle$ we indicate the duality product between a distribution and a smooth function.

Definition 2.26. Let $\mathbf{u} \in W^{1, p}\left(\Omega, \mathbb{R}^{n}\right)$ satisfy $(\operatorname{adj} D \mathbf{u}) \mathbf{u} \in L_{\mathrm{loc}}^{1}\left(\Omega, \mathbb{R}^{n}\right)$. The distributional determinant of $\mathbf{u}$ is the distribution Det $D \mathbf{u}$ defined as

$$
\langle\operatorname{Det} D \mathbf{u}, \phi\rangle:=-\frac{1}{n} \int_{\Omega} \mathbf{u}(\mathbf{x}) \cdot(\operatorname{cof} D \mathbf{u}(\mathbf{x})) D \phi(\mathbf{x}) \mathrm{d} \mathbf{x}, \quad \phi \in C_{c}^{\infty}(\Omega) .
$$

When Det $D \mathbf{u}$ can be identified with a Radon measure or an $L^{1}$ function, we will use that identification without further comment. In particular, equality $\operatorname{Det} D \mathbf{u}=\operatorname{det} D \mathbf{u}$, which can be expressed as (1.1) in the distributional sense, means that

$$
-\frac{1}{n} \int_{\Omega} \mathbf{u}(\mathbf{x}) \cdot(\operatorname{cof} D \mathbf{u}(\mathbf{x})) D \phi(\mathbf{x}) \mathrm{d} \mathbf{x}=\int_{\Omega} \operatorname{det} D \mathbf{u}(\mathbf{x}) \phi(\mathbf{x}) \mathrm{d} \mathbf{x} \quad \text { for all } \phi \in C_{c}^{\infty}(\Omega) .
$$




\section{$3 \quad$ Degree for Sobolev maps}

In this section we recall the definition of the Brouwer degree in $W^{1, p}, p>n-1$; it will play a fundamental role in the proof of the local invertibility and openness properties in the class $\mathcal{A}_{p}$ of sufficiently regular and orientation-preserving Sobolev maps to be defined in Section 4. We then summarize the properties of the degree that are used in our study: on the one hand, the relation of the local degree to the sign of $\operatorname{det} D \mathbf{u}$; on the other hand, that the distributional derivative of the degree is related to cof $D \mathbf{u}$ in the reference configuration. Finally, we state Šverák's [70] definition of the topological image of a set and Müller \& Spector's [61] topological condition of invertibility.

We assume that the reader has some familiarity with the topological degree for continuous functions (see, e.g., $[21,31])$. Let $U$ be a bounded open set of $\mathbb{R}^{n}$ and let $\phi: \partial U \rightarrow \mathbb{R}^{n}$ be continuous. By Tietze's theorem, it admits a continuous extension $\tilde{\phi}: \bar{U} \rightarrow \mathbb{R}^{n}$. We define the degree $\operatorname{deg}(\phi, U, \cdot): \mathbb{R}^{n} \backslash \phi(\partial U) \rightarrow \mathbb{Z}$ of $\boldsymbol{\phi}$ on $U$ as the degree $\operatorname{deg}(\tilde{\phi}, U, \cdot): \mathbb{R}^{n} \backslash \phi(\partial U) \rightarrow \mathbb{Z}$ of $\tilde{\phi}$ on $U$. This definition is consistent since the degree only depends on the boundary values (see, e.g., [21, Th. 3.1 (d6)]).

The following continuity property of the degree is normally proved for functions defined in the whole $\bar{U}$ and not just in $\partial U$.

Lemma 3.1. Let $U$ be a bounded open set of $\mathbb{R}^{n}$, and let $\phi_{1}, \phi_{2} \in C\left(\partial U, \mathbb{R}^{n}\right)$ and $\mathbf{y} \in \mathbb{R}^{n}$ satisfy

$$
\left\|\phi_{1}-\phi_{2}\right\|_{\infty}<\operatorname{dist}\left(\mathbf{y}, \phi_{2}(\partial U)\right) .
$$

Then $\mathbf{y} \notin \boldsymbol{\phi}_{1}(\partial U)$ and $\operatorname{deg}\left(\boldsymbol{\phi}_{1}, U, \mathbf{y}\right)=\operatorname{deg}\left(\boldsymbol{\phi}_{2}, U, \mathbf{y}\right)$.

Proof. Let $\tilde{\phi}_{1}, \tilde{\phi}_{2} \in C\left(\bar{U}, \mathbb{R}^{n}\right)$ be extensions of $\phi_{1}$ and $\phi_{2}$, respectively. Define $H:[0,1] \times \bar{U} \rightarrow \mathbb{R}^{n}$ as

$$
H(t, \mathbf{x}):=t \tilde{\phi}_{1}(\mathbf{x})+(1-t) \tilde{\phi}_{2}(\mathbf{x}) .
$$

Condition (3.1) implies that $\mathbf{y} \notin H([0,1] \times \partial U)$. By the homotopy-invariance of the degree, $\operatorname{deg}\left(\tilde{\boldsymbol{\phi}}_{1}, U, \mathbf{y}\right)=$ $\operatorname{deg}\left(\tilde{\boldsymbol{\phi}}_{2}, U, \mathbf{y}\right)$, and the conclusion follows.

Let $p>n-1$ and $U$ a $C^{1}$ open set of $\mathbb{R}^{n}$ such that $\mathbf{u} \in W^{1, p}\left(\partial U, \mathbb{R}^{n}\right)$. Then $\mathbf{u}$ has a continuous representative, which we still call $\mathbf{u}$ (see Convention 2.14). Hence, $\mathbf{u}$ has a degree $\operatorname{deg}(\mathbf{u}, U, \cdot)$; moreover, $\mathcal{L}^{n}(\mathbf{u}(\partial U))=0$. We will interpret $\operatorname{deg}(\mathbf{u}, U, \cdot)$ in two ways: as a continuous function from $\mathbb{R}^{n} \backslash \mathbf{u}(\partial U)$ to $\mathbb{Z}$, and as a $B V$ function defined a.e. from $\mathbb{R}^{n}$ to $\mathbb{Z}$ (see [61, Lemma 3.5]). In the former case, $\operatorname{deg}(\mathbf{u}, U, \cdot)$ is constant in each connected component of $\mathbb{R}^{n} \backslash \mathbf{u}(\partial U)$. In particular, if $\mathbf{u} \in W^{1, p}\left(\Omega, \mathbb{R}^{n}\right)$ and $\mathbf{x}_{0} \in \Omega$, thanks to the coarea formula,

$$
\mathbf{u} \in W^{1, p}\left(\partial B\left(\mathbf{x}_{0}, r\right), \mathbb{R}^{n}\right)
$$

for a.e. $r \in\left(0, \operatorname{dist}\left(\mathbf{x}_{0}, \partial \Omega\right)\right)$, so $\operatorname{deg}\left(\mathbf{u}, B\left(\mathbf{x}_{0}, r\right), \cdot\right)$ is defined for those $r$, and $\left.\mathbf{u}\right|_{\partial B\left(\mathbf{x}_{0}, r\right)}$ is identified with its continuous representative, according to Convention 2.14.

That the degree of linear invertible maps coincides with the sign of the determinant is a classic result, and so is the formula for the degree of $C^{1}$ maps at regular values (see, e.g., [31, Th. 2.9]). The extension to continuous maps with a regular approximate differential (recall Definition 2.5) was given in [31, Lemma 5.10]. In the next lemma, we extend their result for our situation at hand.

Lemma 3.2. Let $p>n-1, \mathbf{u} \in W^{1, p}\left(\Omega, \mathbb{R}^{n}\right)$ and $\mathbf{x}_{0} \in \Omega$. Assume that $\mathbf{u}$ has a regular approximate differential at $\mathbf{x}_{0}$ with $\operatorname{det} \nabla \mathbf{u}\left(\mathbf{x}_{0}\right) \neq 0$. Then there exists a measurable set $A \subset(0, \infty)$ with $D(A, 0)=\frac{1}{2}$ such that for every $h \in A$,

$$
B\left(\mathbf{x}_{0}, h\right) \in \mathcal{U}_{\mathbf{u}}, \quad \mathbf{u}\left(\mathbf{x}_{0}\right) \notin \mathbf{u}\left(\partial B\left(\mathbf{x}_{0}, h\right)\right) \quad \text { and } \quad \operatorname{deg}\left(\mathbf{u}, B\left(\mathbf{x}_{0}, h\right), \mathbf{u}\left(\mathbf{x}_{0}\right)\right)=\operatorname{sgn} \operatorname{det} \nabla \mathbf{u}\left(\mathbf{x}_{0}\right) .
$$

Moreover, if $h^{\prime}, h \in A$ satisfy $h^{\prime}<\frac{h}{2}$ and $B\left(\mathbf{x}_{0}, h^{\prime}\right) \in \mathcal{U}_{\mathbf{u}}$ then $\mathbf{u}\left(\partial B\left(\mathbf{x}_{0}, h^{\prime}\right)\right)$ is included in the connected component of $\mathbb{R}^{n} \backslash \mathbf{u}\left(\partial B\left(\mathbf{x}_{0}, h\right)\right)$ containing $\mathbf{u}\left(\mathbf{x}_{0}\right)$. 
Proof. Take a set $A \subset \mathbb{R}$ as in Definition 2.5. Thanks to Lemma 2.20, by removing from $A$ a negligible set, it is possible to assume in addition that for every $h \in A$ one has that $B\left(\mathbf{x}_{0}, h\right) \in \mathcal{U}_{\mathbf{u}}$. Define for each $h \in A$,

$$
\varepsilon_{h}:=\sup _{\mathbf{x} \in \partial B\left(\mathbf{x}_{0}, h\right)} \frac{\left|\mathbf{u}(\mathbf{x})-\mathbf{u}\left(\mathbf{x}_{0}\right)-\nabla \mathbf{u}\left(\mathbf{x}_{0}\right)\left(\mathbf{x}-\mathbf{x}_{0}\right)\right|}{\left|\mathbf{x}-\mathbf{x}_{0}\right|}
$$

and $\delta:=\min \left\{\left|\nabla \mathbf{u}\left(\mathbf{x}_{0}\right) \mathbf{z}\right|: \mathbf{z} \in \mathbb{S}^{n-1}\right\}$. As $\operatorname{det} \nabla \mathbf{u}\left(\mathbf{x}_{0}\right) \neq 0$, the quantity $\delta$ is positive. Moreover, recalling Definition 2.5, we can choose an $h_{\mathbf{x}_{0}}>0$ such that $\varepsilon_{h}<\delta / 4$ for any $h \in\left(0, h_{\mathbf{x}_{0}}\right) \cap A$. For simplicity of notation, we rename $A$ as this new set $\left(0, h_{\mathbf{x}_{0}}\right) \cap A$.

Define the affine map $\mathbf{L}: \mathbb{R}^{n} \rightarrow \mathbb{R}^{n}$

$$
\mathbf{L}(\mathbf{x}):=\mathbf{u}\left(\mathbf{x}_{0}\right)+\nabla \mathbf{u}\left(\mathbf{x}_{0}\right)\left(\mathbf{x}-\mathbf{x}_{0}\right) .
$$

For each $h \in A$ and $\mathbf{x} \in \partial B\left(\mathbf{x}_{0}, h\right)$ we have that

$$
\frac{|\mathbf{u}(\mathbf{x})-\mathbf{L}(\mathbf{x})|}{h}=\frac{\left|\mathbf{u}(\mathbf{x})-\mathbf{u}\left(\mathbf{x}_{0}\right)-\nabla \mathbf{u}\left(\mathbf{x}_{0}\right)\left(\mathbf{x}-\mathbf{x}_{0}\right)\right|}{\left|\mathbf{x}-\mathbf{x}_{0}\right|} \leq \varepsilon_{h}
$$

and

$$
\left|\mathbf{L}(\mathbf{x})-\mathbf{u}\left(\mathbf{x}_{0}\right)\right|=\left|\nabla \mathbf{u}\left(\mathbf{x}_{0}\right)\left(\mathbf{x}-\mathbf{x}_{0}\right)\right| \geq \delta h .
$$

Inequalities (3.2) and (3.3) conclude that

$$
\|\mathbf{u}-\mathbf{L}\|_{\infty, \partial B\left(\mathbf{x}_{0}, h\right)} \leq \varepsilon_{h} h<\delta h \leq \operatorname{dist}\left(\mathbf{u}\left(\mathbf{x}_{0}\right), \mathbf{L}\left(\partial B\left(\mathbf{x}_{0}, h\right)\right)\right) .
$$

By Lemma 3.1, $\mathbf{u}\left(\mathbf{x}_{0}\right) \notin \mathbf{u}\left(\partial B\left(\mathbf{x}_{0}, h\right)\right)$ and $\operatorname{deg}\left(\mathbf{u}, B\left(\mathbf{x}_{0}, h\right), \mathbf{u}\left(\mathbf{x}_{0}\right)\right)=\operatorname{deg}\left(\mathbf{L}, B\left(\mathbf{x}_{0}, h\right), \mathbf{u}\left(\mathbf{x}_{0}\right)\right)$. As $\mathbf{L}$ is affine with $\operatorname{det} \nabla \mathbf{u}\left(\mathbf{x}_{0}\right) \neq 0$, it satisfies $\operatorname{deg}\left(\mathbf{L}, B\left(\mathbf{x}_{0}, h\right), \mathbf{u}\left(\mathbf{x}_{0}\right)\right)=\operatorname{sgn} \operatorname{det} \nabla \mathbf{u}\left(\mathbf{x}_{0}\right)$.

Let now $h^{\prime} \in\left(0, \frac{h}{2}\right) \cap A$ satisfy $B\left(\mathbf{x}_{0}, h^{\prime}\right) \in \mathcal{U}_{\mathbf{u}}$. Define the set $D:=\left\{\mathbf{y} \in \mathbb{R}^{n}: \operatorname{dist}\left(\mathbf{y}, \mathbf{L}\left(\bar{B}\left(\mathbf{x}_{0}, h^{\prime}\right)\right)\right) \leq \frac{\delta h}{8}\right\}$, which is convex (and, hence, connected) since so is $\mathbf{L}\left(\bar{B}\left(\mathbf{x}_{0}, h^{\prime}\right)\right)$. By (3.2) applied to $h^{\prime}$ we have that

$$
\|\mathbf{u}-\mathbf{L}\|_{\infty, \partial B\left(\mathbf{x}_{0}, h^{\prime}\right)} \leq \varepsilon_{h^{\prime}} h^{\prime}<\frac{\delta h}{8},
$$

so $\mathbf{u}\left(\partial B\left(\mathbf{x}_{0}, h^{\prime}\right)\right) \subset D$. Now, for any $\mathbf{x}_{1} \in \partial B\left(\mathbf{x}_{0}, h\right)$ and $\mathbf{x}_{2} \in \bar{B}\left(\mathbf{x}_{0}, h^{\prime}\right)$, we get, using (3.2),

$$
\left|\mathbf{u}\left(\mathbf{x}_{1}\right)-\mathbf{L}\left(\mathbf{x}_{2}\right)\right| \geq\left|\mathbf{L}\left(\mathbf{x}_{1}\right)-\mathbf{L}\left(\mathbf{x}_{2}\right)\right|-\left|\mathbf{u}\left(\mathbf{x}_{1}\right)-\mathbf{L}\left(\mathbf{x}_{1}\right)\right| \geq\left|\nabla \mathbf{u}\left(\mathbf{x}_{0}\right)\left(\mathbf{x}_{1}-\mathbf{x}_{2}\right)\right|-\varepsilon_{h} h \geq \frac{\delta h}{2}-\varepsilon_{h} h>\frac{\delta h}{4} .
$$

Therefore, $D \cap \mathbf{u}\left(\partial B\left(\mathbf{x}_{0}, h\right)\right)=\varnothing$. Altogether, the set $D$ is connected and

$$
\mathbf{u}\left(\partial B\left(\mathbf{x}_{0}, h^{\prime}\right)\right) \subset D \subset \mathbb{R}^{n} \backslash \mathbf{u}\left(\partial B\left(\mathbf{x}_{0}, h\right)\right) .
$$

Therefore, $D$ is included in the connected component of $\mathbb{R}^{n} \backslash \mathbf{u}\left(\partial B\left(\mathbf{x}_{0}, h\right)\right)$ containing $\mathbf{u}\left(\mathbf{x}_{0}\right)$.

The following formula for the distributional derivative of the degree will be widely used (see, e.g., [62, Prop. 2.1] or [61, Prop. 2.1]).

Proposition 3.3. Let $U \subset \mathbb{R}^{n}$ be a $C^{1}$ open set. Suppose that $\mathbf{u}$ is the continuous representative of a function in $W^{1, p}\left(\partial U, \mathbb{R}^{n}\right)$. Then, for all $\mathbf{g} \in C^{1}\left(\mathbb{R}^{n}, \mathbb{R}^{n}\right)$,

$$
\int_{\partial U} \mathbf{g}(\mathbf{u}(\mathbf{x})) \cdot(\operatorname{cof} D \mathbf{u}(\mathbf{x}) \boldsymbol{\nu}(\mathbf{x})) \mathrm{d} \mathcal{H}^{n-1}(\mathbf{x})=\int_{\mathbb{R}^{n}} \operatorname{div} \mathbf{g}(\mathbf{y}) \operatorname{deg}(\mathbf{u}, U, \mathbf{y}) \mathrm{d} \mathbf{y},
$$

where $\boldsymbol{\nu}$ is the unit outward normal to $U$.

The concept of topological image was introduced by Šverák [70] (see also [61]). 
Definition 3.4. Let $p>n-1$ and let $U \subset \subset \mathbb{R}^{n}$ be a nonempty open set with a $C^{1}$ boundary. If $\mathbf{u} \in$ $W^{1, p}\left(\partial U, \mathbb{R}^{n}\right)$, we define $\operatorname{im}_{\mathrm{T}}(\mathbf{u}, U)$, the topological image of $U$ under $\mathbf{u}$, as the set of $\mathbf{y} \in \mathbb{R}^{n} \backslash \mathbf{u}(\partial U)$ such that $\operatorname{deg}(\mathbf{u}, U, \mathbf{y}) \neq 0$.

Due to the continuity of the degree for $W^{1, p}$ maps when $p>n-1$, the $\operatorname{set} \operatorname{im}_{\mathrm{T}}(\mathbf{u}, U)$ is open and $\partial \operatorname{im}_{\mathrm{T}}(\mathbf{u}, U) \subset \mathbf{u}(\partial U)$. In addition, as $\operatorname{deg}(\mathbf{u}, U, \cdot)$ is zero in the unbounded component of $\mathbb{R}^{n} \backslash \mathbf{u}(\partial U)(\operatorname{see}$, e.g., [21, Sect. 5.1]), it follows that $\operatorname{im}_{\mathrm{T}}(\mathbf{u}, U)$ is bounded.

Condition INV (see $[61,16])$ is defined as follows.

Definition 3.5. Let $\mathbf{u} \in W^{1, p}\left(\Omega, \mathbb{R}^{n}\right)$ with $p>n-1$. We say that $\mathbf{u}$ satisfies condition INV provided that for every $\mathbf{x}_{0} \in \Omega$ and a.e. $r \in\left(0\right.$, $\left.\operatorname{dist}\left(\mathbf{x}_{0}, \partial \Omega\right)\right)$, the following conditions hold:

a) $\mathbf{u}(\mathbf{x}) \in \operatorname{im}_{\mathrm{T}}\left(\mathbf{u}, B\left(\mathbf{x}_{0}, r\right)\right)$ for a.e. $\mathbf{x} \in B\left(\mathbf{x}_{0}, r\right)$.

b) $\mathbf{u}(\mathbf{x}) \notin \operatorname{im}_{\mathrm{T}}\left(\mathbf{u}, B\left(\mathbf{x}_{0}, r\right)\right)$ for a.e. $\mathbf{x} \in \Omega \backslash B\left(\mathbf{x}_{0}, r\right)$.

The following consequence of Lemma 3.1 will be used several times.

Lemma 3.6. Let $p>n-1$ and let $U \subset \subset \mathbb{R}^{n}$ be a nonempty open set with a $C^{1}$ boundary. For each $j \in \mathbb{N}$, let $\mathbf{u}, \mathbf{u}_{j} \in W^{1, p}\left(\partial U, \mathbb{R}^{n}\right)$ satisfy $\mathbf{u}_{j} \rightarrow \mathbf{u}$ uniformly in $\partial U$ as $j \rightarrow \infty$. Then:

a) For each compact $K \subset \operatorname{im}_{\mathrm{T}}(\mathbf{u}, U)$ there exists $j_{K} \in \mathbb{N}$ such that $K \subset \operatorname{im}_{\mathrm{T}}\left(\mathbf{u}_{j}, U\right)$ for all $j \geq j_{K}$.

b) For each compact $K \subset \mathbb{R}^{n} \backslash\left(\operatorname{im}_{\mathrm{T}}(\mathbf{u}, U) \cup \mathbf{u}(\partial U)\right)$ there exists $j_{K} \in \mathbb{N}$ such that $K \subset \mathbb{R}^{n} \backslash\left(\operatorname{im}_{\mathrm{T}}\left(\mathbf{u}_{j}, U\right) \cup\right.$ $\left.\mathbf{u}_{j}(\partial U)\right)$ for all $j \geq j_{K}$.

c) $\chi_{\mathrm{im}_{\mathrm{T}}\left(\mathbf{u}_{j}, U\right)} \rightarrow \chi_{\mathrm{im}_{\mathrm{T}}(\mathbf{u}, U)}$ a.e. and in $L^{1}\left(\mathbb{R}^{n}\right)$ as $j \rightarrow \infty$.

Proof. For part a), let $j_{K} \in \mathbb{N}$ be such that for all $j \geq j_{K}$,

$$
\left\|\mathbf{u}_{j}-\mathbf{u}\right\|_{\infty}<\operatorname{dist}\left(K, \mathbb{R}^{n} \backslash \operatorname{im}_{\mathrm{T}}(\mathbf{u}, U)\right) .
$$

By Lemma 3.1, any $\mathbf{y} \in K$ satisfies $\mathbf{y} \notin \mathbf{u}_{j}(\partial U)$ and $\operatorname{deg}\left(\mathbf{u}_{j}, U, \mathbf{y}\right)=\operatorname{deg}(\mathbf{u}, U, \mathbf{y}) \neq 0$ for all $j \geq j_{K}$, so $\mathbf{y} \in \operatorname{im}_{\mathrm{T}}\left(\mathbf{u}_{j}, U\right)$.

For part b), notice that, by the continuity of the degree, $\operatorname{im}_{\mathrm{T}}(\mathbf{u}, U) \cup \mathbf{u}(\partial U)$ is closed. Let $j_{K} \in \mathbb{N}$ be such that for all $j \geq j_{K}$,

$$
\left\|\mathbf{u}_{j}-\mathbf{u}\right\|_{\infty}<\operatorname{dist}\left(K, \operatorname{im}_{\mathrm{T}}(\mathbf{u}, U) \cup \mathbf{u}(\partial U)\right)
$$

By Lemma 3.1, any $\mathbf{y} \in K$ satisfies $\mathbf{y} \notin \mathbf{u}_{j}(\partial U)$ and $\operatorname{deg}\left(\mathbf{u}_{j}, U, \mathbf{y}\right)=\operatorname{deg}(\mathbf{u}, U, \mathbf{y})=0$ for all $j \geq j_{K}$, so $\mathbf{y} \notin \operatorname{im}_{\mathrm{T}}\left(\mathbf{u}_{j}, U\right)$.

Parts $a)$ and $b$ ) show that $\chi_{\mathrm{im}_{\mathrm{T}}\left(\mathbf{u}_{j}, U\right)} \rightarrow \chi_{\mathrm{im}_{\mathrm{T}}(\mathbf{u}, U)}$ pointwise in $\mathbb{R}^{n} \backslash \mathbf{u}(\partial U)$, hence a.e. thanks to Remark $2.16 \mathrm{~b})$. Now, $\chi_{\mathrm{im}_{\mathrm{T}}\left(\mathbf{u}_{j}, U\right)}=0$ in the unbounded connected component of $\mathbb{R}^{n} \backslash \mathbf{u}_{j}(\partial U)$, for each $j \in \mathbb{N}$. Therefore, as $\bigcup_{j \in \mathbb{N}} \mathbf{u}_{j}(\partial U)$ is bounded, so is $\bigcup_{j \in \mathbb{N}} \operatorname{im}_{\mathrm{T}}\left(\mathbf{u}_{j}, B\right)$. By dominated convergence, the convergence of c) also holds in $L^{1}\left(\mathbb{R}^{n}\right)$.

\section{Local invertibility and preservation of orientation}

In [44, Sect. 7], an example was shown of a one-to-one a.e. map $\mathbf{u} \in L^{\infty}\left(\Omega, \mathbb{R}^{n}\right)(n=2)$ satisfying $\mathbf{u} \in$ $W^{1, p}\left(\Omega, \mathbb{R}^{n}\right)$ for all $p<n, \operatorname{Det} D \mathbf{u}=\operatorname{det} D \mathbf{u}$, $\operatorname{det} D \mathbf{u}>0$ a.e. and $\mathcal{E}(\mathbf{u})>0$. The result seems contradictory since, for invertible maps, $\mathcal{E}(\mathbf{u})$ measures the area of the cavity surfaces created by $\mathbf{u}$ (see [44, Th. 4.6]), and, for orientation-preserving maps, the difference between Det $D \mathbf{u}$ and det $D \mathbf{u}$ gives the location of the cavitation singularities together with the volume of the created cavities (see [61, Th. 8.4] or [44, Th. 6.2]). What happens in that construction is that, when $p<n$, the pointwise condition det $D \mathbf{u}>0$ a.e. proves insufficient to prevent a reversal of orientation: this reversal takes place at the surface of a created cavity in the deformed configuration, which corresponds to an isolated point in the reference configuration. The lesson we draw is that, in our context, the notion of preservation of orientation should be expressed in terms 
of the non-negativity of the degree. In particular, under this more accurate assumption, we are able to show in this section, by proving Theorem 1.1 , that the conditions $\operatorname{Det} D \mathbf{u}=\operatorname{det} D \mathbf{u}$ and $\mathcal{E}(\mathbf{u})=0$ are indeed equivalent. This turns out to be intrinsically related to the fact that, under any of these conditions, $\mathbf{u}$ is locally invertible a.e.

We start by proving the easier implication, i.e., $\mathcal{E}(\mathbf{u})=0$ implies Det $D \mathbf{u}=\operatorname{det} D \mathbf{u}$. Recall the notation $\mathcal{N}$ from Section 2.3.

Theorem 4.1. Let $\mathbf{u} \in W^{1, p}\left(\Omega, \mathbb{R}^{n}\right), p>n-1$, $\operatorname{det} D \mathbf{u} \in L_{\mathrm{loc}}^{1}(\Omega), \mathcal{E}(\mathbf{u})=0$ and $\operatorname{det}$ Du $>0$ a.e. Then $\mathbf{u} \in L_{\mathrm{loc}}^{\infty}\left(\Omega, \mathbb{R}^{n}\right)$, Det $D \mathbf{u}=\operatorname{det} D \mathbf{u}$ and, for all $U \in \mathcal{U}_{\mathbf{u}}$,

$$
\operatorname{deg}(\mathbf{u}, U, \cdot)=\mathcal{N}_{U} \text { a.e. }
$$

Proof. Let $U \in \mathcal{U}_{\mathbf{u}}$ and let $\left\{\phi_{\delta}\right\}_{\delta>0}$ be the family of Proposition 2.23. Fix $\mathbf{g} \in C_{c}^{1}\left(\mathbb{R}^{n}, \mathbb{R}^{n}\right)$ and let $\boldsymbol{\nu}$ be the outward normal to $U$. Using Proposition 3.3 as well, we find that

$$
\begin{aligned}
\int_{\mathbb{R}^{n}} \operatorname{div} \mathbf{g}(\mathbf{y}) \operatorname{deg}(\mathbf{u}, U, \mathbf{y}) \mathrm{d} \mathbf{y} & =\int_{\partial U} \mathbf{g}(\mathbf{u}(\mathbf{x})) \cdot(\operatorname{cof} D \mathbf{u}(\mathbf{x}) \boldsymbol{\nu}(\mathbf{x})) \mathrm{d} \mathcal{H}^{n-1}(\mathbf{x}) \\
& =-\lim _{\delta \searrow 0} \int_{\Omega} \mathbf{g}(\mathbf{u}(\mathbf{x})) \cdot\left(\operatorname{cof} D \mathbf{u}(\mathbf{x}) D \phi_{\delta}(\mathbf{x})\right) \mathrm{d} \mathbf{x} .
\end{aligned}
$$

Now, using $\mathcal{E}(\mathbf{u})=0$ in the form (2.4), dominated convergence and Proposition 2.7, we find that

$$
\begin{aligned}
-\lim _{\delta \searrow 0} \int_{\Omega} \mathbf{g}(\mathbf{u}(\mathbf{x})) \cdot\left(\operatorname{cof} D \mathbf{u}(\mathbf{x}) D \phi_{\delta}(\mathbf{x})\right) \mathrm{d} \mathbf{x} & =\lim _{\delta \searrow 0} \int_{\Omega} \phi_{\delta}(\mathbf{x}) \operatorname{div} \mathbf{g}(\mathbf{u}(\mathbf{x})) \operatorname{det} D \mathbf{u}(\mathbf{x}) \mathrm{d} \mathbf{x} \\
& =\int_{U} \operatorname{div} \mathbf{g}(\mathbf{u}(\mathbf{x})) \operatorname{det} D \mathbf{u}(\mathbf{x}) \mathrm{d} \mathbf{x}=\int_{\mathbb{R}^{n}} \operatorname{div} \mathbf{g}(\mathbf{y}) \mathcal{N}_{U}(\mathbf{y}) \mathrm{d} \mathbf{y}
\end{aligned}
$$

From (4.2) and (4.3) we obtain that

$$
\int_{\mathbb{R}^{n}} \operatorname{div} \mathbf{g}(\mathbf{y}) \operatorname{deg}(\mathbf{u}, U, \mathbf{y}) \mathrm{d} \mathbf{y}=\int_{\mathbb{R}^{n}} \operatorname{div} \mathbf{g}(\mathbf{y}) \mathcal{N}_{U}(\mathbf{y}) \mathrm{d} \mathbf{y} .
$$

Therefore, there exists $c \in \mathbb{Z} \operatorname{such}$ that $\mathcal{N}_{U}-\operatorname{deg}(\mathbf{u}, U, \cdot)=c$ a.e.

Now we show that $\operatorname{im}_{\mathrm{G}}(\mathbf{u}, U) \subset \operatorname{im}_{\mathrm{T}}(\mathbf{u}, U)$ a.e. For all $\mathbf{y} \in \operatorname{im}_{\mathrm{G}}(\mathbf{u}, U) \backslash\left(\operatorname{im}_{\mathrm{T}}(\mathbf{u}, U) \cup \mathbf{u}(\partial U)\right)$ we have $\mathcal{N}_{U}(\mathbf{y}) \geq 1$ and $\operatorname{deg}(\mathbf{u}, U, \mathbf{y})=0$, so if

$$
\mathcal{L}^{n}\left(\operatorname{im}_{\mathrm{G}}(\mathbf{u}, U) \backslash \operatorname{im}_{\mathrm{T}}(\mathbf{u}, U)\right)>0
$$

we would obtain $c \geq 1$. Thus, for a.e. $\mathbf{y} \in \mathbb{R}^{n} \backslash \operatorname{im}_{\mathrm{T}}(\mathbf{u}, U)$ we would have $\operatorname{deg}(\mathbf{u}, U, \mathbf{y})=0$, so $\mathcal{N}_{U}(\mathbf{y})=c \geq 1$ and, hence, $\mathbf{y} \in \operatorname{im}_{\mathrm{G}}(\mathbf{u}, U)$. Therefore, $\mathbb{R}^{n} \backslash \operatorname{im}_{\mathrm{T}}(\mathbf{u}, U) \subset \operatorname{im}_{\mathrm{G}}(\mathbf{u}, U)$ a.e., so, using that im $(\mathbf{u}, U)$ is bounded, as well as Proposition 2.7,

$$
\infty=\mathcal{L}^{n}\left(\mathbb{R}^{n} \backslash \operatorname{im}_{\mathrm{T}}(\mathbf{u}, U)\right) \leq \mathcal{L}^{n}\left(\operatorname{im}_{\mathrm{G}}(\mathbf{u}, U)\right) \leq \int_{\operatorname{im}_{\mathrm{G}}(\mathbf{u}, U)} \mathcal{N}_{U}(\mathbf{y}) \mathrm{d} \mathbf{y}=\int_{U} \operatorname{det} D \mathbf{u}(\mathbf{x}) \mathrm{d} \mathbf{x},
$$

which contradicts the fact that $\operatorname{det} D \mathbf{u} \in L_{\mathrm{loc}}^{1}(\Omega)$; this contradiction comes from assumption (4.4). Thus, $\operatorname{im}_{\mathrm{G}}(\mathbf{u}, U) \subset \operatorname{im}_{\mathrm{T}}(\mathbf{u}, U)$ a.e.; consequently, $\operatorname{im}_{\mathrm{G}}(\mathbf{u}, U)$ is essentially bounded and $\mathbf{u} \in L^{\infty}\left(U, \mathbb{R}^{n}\right)$. Therefore, $\mathbf{u} \in L_{\mathrm{loc}}^{\infty}\left(\Omega, \mathbb{R}^{n}\right)$.

Now, for a.e. $\mathbf{y} \in \mathbb{R}^{n} \backslash \operatorname{im}_{\mathrm{T}}(\mathbf{u}, U)$ we have $\operatorname{deg}(\mathbf{u}, U, \mathbf{y})=0, \mathbf{y} \notin \operatorname{im}_{\mathrm{G}}(\mathbf{u}, U)$ and, hence, $\mathcal{N}_{U}(\mathbf{y})=0$. Thus $c=0$ and, hence, $\operatorname{deg}(\mathbf{u}, U, \cdot)=\mathcal{N}_{U}$ a.e.

Now let $\phi \in C_{c}^{1}(\Omega)$ and choose, thanks to Lemma 2.20, $U \in \mathcal{U}_{\mathbf{u}}$ such that $\operatorname{spt} \phi \subset U$. As $\operatorname{im}_{\mathrm{G}}(\mathbf{u}, U)$ is essentially bounded, we can find $\mathbf{g} \in C_{c}^{1}\left(\mathbb{R}^{n}, \mathbb{R}^{n}\right)$ such that $\mathbf{g}=-\frac{1}{n} \mathbf{i d}$ a.e. in $\operatorname{im}_{\mathrm{G}}(\mathbf{u}, U)$. Using again $(2.4)$, we obtain that

$$
\begin{aligned}
\langle\operatorname{Det} D \mathbf{u}, \phi\rangle & =-\frac{1}{n} \int_{\Omega} \mathbf{u}(\mathbf{x}) \cdot(\operatorname{cof} D \mathbf{u}(\mathbf{x}) D \phi(\mathbf{x})) \mathrm{d} \mathbf{x}=\int_{\Omega} \mathbf{g}(\mathbf{u}(\mathbf{x})) \cdot(\operatorname{cof} D \mathbf{u}(\mathbf{x}) D \phi(\mathbf{x})) \mathrm{d} \mathbf{x} \\
& =-\int_{\Omega} \phi(\mathbf{x}) \operatorname{div} \mathbf{g}(\mathbf{u}(\mathbf{x})) \operatorname{det} D \mathbf{u}(\mathbf{x}) \mathrm{d} \mathbf{x}=\int_{\Omega} \phi(\mathbf{x}) \operatorname{det} D \mathbf{u}(\mathbf{x}) \mathrm{d} \mathbf{x}
\end{aligned}
$$

which shows that $\operatorname{Det} D \mathbf{u}=\operatorname{det} D \mathbf{u}$. 
In order to prove the reverse implication, i.e., $\operatorname{Det} D \mathbf{u}=\operatorname{det} D \mathbf{u}$ implies $\mathcal{E}(\mathbf{u})=0$ when the topological degree is non-negative, we need some preliminary results. We start by fixing a suitable family of mollifiers. Let $\psi \in C^{1}([0, \infty))$ be such that

$$
\psi \geq 0, \quad \psi^{\prime} \leq 0, \quad \psi^{\prime}(0)=0, \quad \operatorname{spt} \psi \subset[0,1), \quad \int_{\mathbb{R}^{n}} \psi(|\mathbf{x}|) \mathrm{d} \mathbf{x}=1
$$

and, for $t>0$, define $\psi_{t} \in C^{1}(\mathbb{R})$ and $\varphi_{t} \in C^{1}\left(\mathbb{R}^{n}\right)$ as

$$
\psi_{t}(r):=\frac{1}{t^{n}} \psi\left(\frac{r}{t}\right), \quad \varphi_{t}(\mathbf{x}):=\psi_{t}(|\mathbf{x}|) .
$$

The following result is essentially contained in the proof of [61, Lemma 8.1].

Lemma 4.2. Let $\mathbf{u} \in W^{1, p}\left(\Omega, \mathbb{R}^{n}\right)$ with $p>n-1$. Let $\phi \in C_{c}^{1}(\Omega)$ and $\mathbf{g} \in C^{1}\left(\mathbb{R}^{n}, \mathbb{R}^{n}\right)$. Assume that $(\operatorname{adj} D \mathbf{u})(\mathbf{g} \circ \mathbf{u}) \in L_{\text {loc }}^{1}\left(\Omega, \mathbb{R}^{n}\right)$. Then

$$
\int_{\Omega} \mathbf{g}(\mathbf{u}(\mathbf{x})) \cdot(\operatorname{cof} D \mathbf{u}(\mathbf{x}) D \phi(\mathbf{x})) \mathrm{d} \mathbf{x}=\lim _{t \searrow 0} \int_{\Omega} \phi(\mathbf{x}) \int_{0}^{t} \psi_{t}^{\prime}(r) \int_{\mathbb{R}^{n}} \operatorname{div} \mathbf{g}(\mathbf{y}) \operatorname{deg}(\mathbf{u}, B(\mathbf{x}, r), \mathbf{y}) \mathrm{d} \mathbf{y} \mathrm{d} r \mathrm{~d} \mathbf{x} .
$$

Proof. Denoting by $\star$ the convolution operator, we find that $\phi \star D \varphi_{t} \rightarrow D \phi$ uniformly in $\Omega$ as $t \searrow 0$, since $\phi \in C_{c}^{1}(\Omega)$. Therefore,

$$
\begin{aligned}
\int_{\Omega} \mathbf{g}(\mathbf{u}(\mathbf{x})) \cdot(\operatorname{cof} D \mathbf{u}(\mathbf{x}) D \phi(\mathbf{x})) \mathrm{d} \mathbf{x} & =\int_{\Omega}(\operatorname{adj} D \mathbf{u}(\mathbf{x}) \mathbf{g}(\mathbf{u}(\mathbf{x}))) \cdot D \phi(\mathbf{x}) \mathrm{d} \mathbf{x} \\
& =\lim _{t \searrow 0} \int_{\Omega}(\operatorname{adj} D \mathbf{u}(\mathbf{x}) \mathbf{g}(\mathbf{u}(\mathbf{x}))) \cdot\left(\phi \star D \varphi_{t}\right)(\mathbf{x}) \mathrm{d} \mathbf{x} .
\end{aligned}
$$

Now, for $0<t<\operatorname{dist}(\operatorname{spt} \phi, \partial \Omega)$,

$$
\int_{\Omega}(\operatorname{adj} D \mathbf{u}(\mathbf{x}) \mathbf{g}(\mathbf{u}(\mathbf{x}))) \cdot\left(\phi \star D \varphi_{t}\right)(\mathbf{x}) \mathrm{d} \mathbf{x}=\int_{\operatorname{spt} \phi} \phi(\mathbf{z}) \int_{B(\mathbf{z}, t)}(\operatorname{adj} D \mathbf{u}(\mathbf{x}) \mathbf{g}(\mathbf{u}(\mathbf{x}))) \cdot D \varphi_{t}(\mathbf{x}-\mathbf{z}) \mathrm{d} \mathbf{x} \mathrm{d} \mathbf{z} .
$$

By the coarea formula and (4.5), for each $\mathbf{z} \in \operatorname{spt} \phi$,

$$
\int_{B(\mathbf{z}, r)}(\operatorname{adj} D \mathbf{u}(\mathbf{x}) \mathbf{g}(\mathbf{u}(\mathbf{x}))) \cdot D \varphi_{t}(\mathbf{x}-\mathbf{z}) \mathrm{d} \mathbf{x}=\int_{0}^{t} \psi_{t}^{\prime}(r) \int_{\partial B(\mathbf{z}, r)}(\operatorname{adj} D \mathbf{u}(\mathbf{x}) \mathbf{g}(\mathbf{u}(\mathbf{x}))) \cdot \boldsymbol{\nu}(\mathbf{x}) \mathrm{d} \mathcal{H}^{n-1}(\mathbf{x}) \mathrm{d} r
$$

where $\boldsymbol{\nu}(\mathbf{x}):=\frac{\mathbf{x}-\mathbf{z}}{|\mathbf{x}-\mathbf{z}|}$ is the unit exterior normal to $B(\mathbf{z}, r)$. By Proposition 3.3, for a.e. $r \in(0, t)$,

$$
\begin{aligned}
\int_{\partial B(\mathbf{z}, r)}(\operatorname{adj} D \mathbf{u}(\mathbf{x}) \mathbf{g}(\mathbf{u}(\mathbf{x}))) \cdot \boldsymbol{\nu}(\mathbf{x}) \mathrm{d} \mathcal{H}^{n-1}(\mathbf{x}) & =\int_{\partial B(\mathbf{z}, r)} \mathbf{g}(\mathbf{u}(\mathbf{x})) \cdot(\operatorname{cof} D \mathbf{u}(\mathbf{x}) \boldsymbol{\nu}(\mathbf{x})) \mathrm{d} \mathcal{H}^{n-1}(\mathbf{x}) \\
& =\int_{\mathbb{R}^{n}} \operatorname{div} \mathbf{g}(\mathbf{y}) \operatorname{deg}(\mathbf{u}, B(\mathbf{z}, r), \mathbf{y}) \mathrm{d} \mathbf{y} .
\end{aligned}
$$

This chain of equalities concludes the proof.

The following proposition shows that an important part of the analysis of the distributional determinant carried out in [61, Sect. 8] (in particular, the monotonicity of the topological images) does not depend on the invertibility of the deformations but only on the preservation of orientation (the positivity of the degree).

Proposition 4.3. Let $p>n-1$ and $\mathbf{u} \in W^{1, p}\left(\Omega, \mathbb{R}^{n}\right)$. Assume that (adj Du) $\mathbf{u} \in L_{\text {loc }}^{1}\left(\Omega, \mathbb{R}^{n}\right)$ and that $\operatorname{deg}(\mathbf{u}, B(\mathbf{x}, r), \cdot) \geq 0$ a.e. for all $\mathbf{x} \in \Omega$ and $r>0$ for which $B(\mathbf{x}, r) \in \mathcal{U}_{\mathbf{u}}$. Then

a) Det Du is a positive Radon measure. 
b) $\operatorname{det} D \mathbf{u} \in L_{\text {loc }}^{1}(\Omega)$, $\operatorname{det} D \mathbf{u} \geq 0$ a.e. and $\operatorname{Det} D \mathbf{u}=(\operatorname{det} D \mathbf{u}) \mathcal{L}^{n}+\mu^{s}$ for some measure $\mu^{s}$ that is singular with respect to the Lebesgue measure.

c) For every $U \in \mathcal{U}_{\mathbf{u}}$ we have $\operatorname{deg}(\mathbf{u}, U, \cdot) \geq 0$ a.e. and in $\mathbb{R}^{n} \backslash \mathbf{u}(\partial U)$.

d) For every $U_{1}, U_{2} \in \mathcal{U}_{\mathbf{u}}$ with $U_{1} \subset \subset U_{2}$,

$$
\operatorname{deg}\left(\mathbf{u}, U_{1}, \cdot\right) \leq \operatorname{deg}\left(\mathbf{u}, U_{2}, \cdot\right) \text { a.e. and in } \mathbb{R}^{n} \backslash \mathbf{u}\left(\partial U_{1} \cup \partial U_{2}\right), \quad \text { and } \overline{\overline{\operatorname{im}_{\mathrm{T}}\left(\mathbf{u}, U_{1}\right)}} \subset \overline{\operatorname{im}_{\mathrm{T}}\left(\mathbf{u}, U_{2}\right)} .
$$

e) $\mathbf{u} \in L_{\mathrm{loc}}^{\infty}\left(\Omega, \mathbb{R}^{n}\right)$ and for every $U \in \mathcal{U}_{\mathbf{u}}$ we have $\operatorname{im}_{\mathrm{G}}(\mathbf{u}, U) \subset \operatorname{im}_{\mathrm{T}}(\mathbf{u}, U)$ a.e. and $\operatorname{im}_{\mathrm{G}}(\mathbf{u}, U) \subset \overline{\operatorname{im}_{\mathrm{T}}(\mathbf{u}, U)}$.

f) If, in addition $\operatorname{Det} D \mathbf{u}=\operatorname{det} D \mathbf{u}$, then, for every $U \in \mathcal{U}_{\mathbf{u}}$ and every family $\mathcal{F}$ of balls contained in $U$ and such that, for a.e. $\mathbf{x} \in U$,

$$
\bar{D}(\{r>0: B(\mathbf{x}, r) \in \mathcal{F}\}, 0)>0,
$$

there exists a countable family $\left\{B_{k}\right\}_{k \in \mathbb{N}} \subset \mathcal{F} \cap \mathcal{U}_{\mathbf{u}}$ such that

$$
\bar{B}_{i} \cap \bar{B}_{j}=\varnothing \quad \text { for } i \neq j, \quad U=\bigcup_{k \in \mathbb{N}} B_{k} \quad \text { a.e. } \quad \text { and } \quad \operatorname{deg}(\mathbf{u}, U, \cdot)=\sum_{k \in \mathbb{N}} \operatorname{deg}\left(\mathbf{u}, B_{k}, \cdot\right) \quad \text { a.e. }
$$

Proof. Parts a), c) and f) of the proof proceed as in [61, Th. 9.1].

Part a). For each $\mathbf{g} \in C^{1}\left(\mathbb{R}^{n}, \mathbb{R}^{n}\right)$ such that $(\operatorname{adj} D \mathbf{u})(\mathbf{g} \circ \mathbf{u}) \in L_{\text {loc }}^{1}\left(\Omega, \mathbb{R}^{n}\right)$, define the linear functional $\Lambda_{\mathrm{g}}$ as

$$
\left\langle\Lambda_{\mathbf{g}}, \phi\right\rangle:=-\int_{\Omega} \mathbf{g}(\mathbf{u}(\mathbf{x})) \cdot(\operatorname{cof} D \mathbf{u}(\mathbf{x}) D \phi(\mathbf{x})) \mathrm{d} \mathbf{x}, \quad \phi \in C_{c}^{1}(\Omega) .
$$

If $\operatorname{div} \mathbf{g} \geq 0$, then Lemma 4.2 and the assumption on the degree imply that $\left\langle\Lambda_{\mathbf{g}}, \phi\right\rangle \geq 0$ for all $\phi \in C_{c}^{1}(\Omega)$ with $\phi \geq 0$. Hence (see, e.g., [29, Cor. 1.8.1]) $\Lambda_{\mathbf{g}}$ is a positive measure in $\Omega$. In particular, since (adj $\left.D \mathbf{u}\right) \mathbf{u} \in$ $L_{\text {loc }}^{1}\left(\Omega, \mathbb{R}^{n}\right)$, we can apply this result to $\mathbf{g}:=\frac{1}{n} \mathbf{i d}$ and conclude that $\operatorname{Det} D \mathbf{u}$ is a positive measure. This proves part $a$ ).

Part c). Suppose now that $\mathbf{g}$ is any vector field in $C^{1}\left(\mathbb{R}^{n}, \mathbb{R}^{n}\right)$ such that $(\operatorname{adj} D \mathbf{u})(\mathbf{g} \circ \mathbf{u}) \in L_{\text {loc }}^{1}\left(\Omega, \mathbb{R}^{n}\right)$ and $\operatorname{div} \mathbf{g} \in L^{\infty}\left(\mathbb{R}^{n}\right)$. Call $c_{\mathbf{g}}:=\frac{1}{n} \inf \operatorname{div} \mathbf{g}$ and consider $\overline{\mathbf{g}}:=\mathbf{g}-c_{\mathbf{g}}$ id. Note that $\operatorname{div} \overline{\mathbf{g}} \geq 0$ and $\Lambda_{\overline{\mathbf{g}}}=$ $\Lambda_{\mathrm{g}}-n c_{\mathrm{g}}$ Det $D \mathbf{u}$. By the result above, $\Lambda_{\overline{\mathrm{g}}}$ is a positive measure and, hence, $\Lambda_{\mathrm{g}}$ is a Radon measure in $\Omega$ with

$$
\left|\Lambda_{\mathbf{g}}\right| \leq \Lambda_{\overline{\mathbf{g}}}+n\left|c_{\mathbf{g}}\right| \operatorname{Det} D \mathbf{u} .
$$

Applying twice Lemma 4.2, first to $\overline{\mathbf{g}}$ and then to $-\frac{1}{n} \mathbf{i d}$, we obtain that, for any $\phi \in C_{c}^{1}(\Omega)$ with $\phi \geq 0$,

$$
\left\langle\Lambda_{\overline{\mathbf{g}}}, \phi\right\rangle \leq-\|\operatorname{div} \overline{\mathbf{g}}\|_{\infty} \lim _{t \searrow 0} \int_{\Omega} \phi(\mathbf{x}) \int_{0}^{t} \psi_{t}^{\prime}(r) \int_{\mathbb{R}^{n}} \operatorname{deg}(\mathbf{u}, B(\mathbf{x}, r), \mathbf{y}) \mathrm{d} \mathbf{y} \mathrm{d} r \mathrm{~d} \mathbf{x}=\|\operatorname{div} \overline{\mathbf{g}}\|_{\infty}\langle\operatorname{Det} D \mathbf{u}, \phi\rangle .
$$

This shows that $\Lambda_{\overline{\mathbf{g}}} \leq\|\operatorname{div} \overline{\mathbf{g}}\|_{\infty}$ Det $D \mathbf{u}$. Since $|\operatorname{div} \overline{\mathbf{g}}| \leq|\operatorname{div} \mathbf{g}|+n\left|c_{\mathbf{g}}\right|$, thanks to (4.7), we obtain that

$$
\left|\Lambda_{\mathbf{g}}\right| \leq\left(\|\operatorname{div} \mathbf{g}\|_{\infty}+2 n\left|c_{\mathbf{g}}\right|\right) \operatorname{Det} D \mathbf{u}
$$

Let $U \in \mathcal{U}_{\mathbf{u}}$ and let $\mathcal{F}_{U}$ be the family of closed balls $\bar{B}$ contained in $U$ such that $B \in \mathcal{U}_{\mathbf{u}}$ and Det $D \mathbf{u}(\partial B)=$ 0 . As Det $D \mathbf{u}$ is finite on compact sets, $\mathcal{F}_{U}$ is a fine covering of $U$. By Besicovitch's covering theorem (see, e.g., [2, Th. 2.19]), there exists a countable family $\left\{B_{k}\right\}_{k \in \mathbb{N}}$ of open balls in $\mathcal{U}_{\mathbf{u}}$ with disjoint closures such that

$$
\operatorname{Det} D \mathbf{u}\left(\bigcup_{k \in \mathbb{N}} \partial B_{k}\right)=0 \quad \text { and } \quad\left(\mathcal{L}^{n}+\operatorname{Det} D \mathbf{u}\right)\left(U \backslash \bigcup_{k \in \mathbb{N}} B_{k}\right)=0 .
$$

Let $\left\{\phi_{\delta}\right\}_{\delta>0}$ be the family of Proposition 2.23. Then, for each $\mathbf{g} \in C^{1}\left(\mathbb{R}^{n}, \mathbb{R}^{n}\right)$ for which $(\operatorname{adj} D \mathbf{u})(\mathbf{g} \circ \mathbf{u}) \in$ $L_{\text {loc }}^{1}\left(\Omega, \mathbb{R}^{n}\right)$ and $\operatorname{div} \mathbf{g} \in L^{\infty}\left(\mathbb{R}^{n}\right)$,

$$
\int_{\partial U} \mathbf{g}(\mathbf{u}(\mathbf{x})) \cdot(\operatorname{cof} D \mathbf{u}(\mathbf{x}) \boldsymbol{\nu}(\mathbf{x})) \mathrm{d} \mathcal{H}^{n-1}(\mathbf{x})=\lim _{\delta \searrow 0}\left\langle\Lambda_{\mathbf{g}}, \phi_{\delta}\right\rangle=\Lambda_{\mathbf{g}}(U),
$$


where $\boldsymbol{\nu}$ is the outward normal to $U$. Thus, thanks to Proposition 3.3, (4.8) and (4.9),

$$
\int_{\mathbb{R}^{n}} \operatorname{div} \mathbf{g}(\mathbf{y}) \operatorname{deg}(\mathbf{u}, U, \mathbf{y}) \mathrm{d} \mathbf{y}=\Lambda_{\mathbf{g}}(U)=\sum_{k \in \mathbb{N}} \Lambda_{\mathbf{g}}\left(B_{k}\right)=\sum_{k \in \mathbb{N}} \int_{\mathbb{R}^{n}} \operatorname{div} \mathbf{g}(\mathbf{y}) \operatorname{deg}\left(\mathbf{u}, B_{k}, \mathbf{y}\right) \mathrm{d} \mathbf{y} .
$$

When we apply (4.10) to $\overline{\mathbf{g}}:=\mathbf{g}-c_{\mathbf{g}}$ id, we obtain, using monotone convergence, that

$$
\int_{\mathbb{R}^{n}} \operatorname{div} \overline{\mathbf{g}}(\mathbf{y}) \operatorname{deg}(\mathbf{u}, U, \mathbf{y}) \mathrm{d} \mathbf{y}=\sum_{k \in \mathbb{N}} \int_{\mathbb{R}^{n}} \operatorname{div} \overline{\mathbf{g}}(\mathbf{y}) \operatorname{deg}\left(\mathbf{u}, B_{k}, \mathbf{y}\right) \mathrm{d} \mathbf{y}=\int_{\mathbb{R}^{n}} \operatorname{div} \overline{\mathbf{g}}(\mathbf{y}) \sum_{k \in \mathbb{N}} \operatorname{deg}\left(\mathbf{u}, B_{k}, \mathbf{y}\right) \mathrm{d} \mathbf{y}
$$

whereas when we apply it to $\frac{1}{n} \mathbf{i d}$, we obtain, again by monotone convergence,

$$
\int_{\mathbb{R}^{n}} \operatorname{deg}(\mathbf{u}, U, \mathbf{y}) \mathrm{d} \mathbf{y}=\sum_{k \in \mathbb{N}} \int_{\mathbb{R}^{n}} \operatorname{deg}\left(\mathbf{u}, B_{k}, \mathbf{y}\right) \mathrm{d} \mathbf{y}=\int_{\mathbb{R}^{n}} \sum_{k \in \mathbb{N}} \operatorname{deg}\left(\mathbf{u}, B_{k}, \mathbf{y}\right) \mathrm{d} \mathbf{y} .
$$

In particular, $\sum_{k \in \mathbb{N}} \operatorname{deg}\left(\mathbf{u}, B_{k}, \cdot\right) \in L^{1}(\Omega)$ and

$$
n c_{\mathbf{g}} \int_{\mathbb{R}^{n}} \operatorname{deg}(\mathbf{u}, U, \mathbf{y}) \mathrm{d} \mathbf{y}=n c_{\mathbf{g}} \int_{\mathbb{R}^{n}} \sum_{k \in \mathbb{N}} \operatorname{deg}\left(\mathbf{u}, B_{k}, \mathbf{y}\right) \mathrm{d} \mathbf{y} .
$$

Adding the equalities (4.11) and (4.13), we get

$$
\int_{\mathbb{R}^{n}} \operatorname{div} \mathbf{g}(\mathbf{y}) \operatorname{deg}(\mathbf{u}, U, \mathbf{y}) \mathrm{d} \mathbf{y}=\int_{\mathbb{R}^{n}} \operatorname{div} \mathbf{g}(\mathbf{y}) \sum_{k \in \mathbb{N}} \operatorname{deg}\left(\mathbf{u}, B_{k}, \mathbf{y}\right) \mathrm{d} \mathbf{y} .
$$

This equality is true for all $\mathbf{g} \in C^{1}\left(\mathbb{R}^{n}, \mathbb{R}^{n}\right)$ for which $(\operatorname{adj} D \mathbf{u})(\mathbf{g} \circ \mathbf{u}) \in L_{\text {loc }}^{1}\left(\Omega, \mathbb{R}^{n}\right)$ and $\operatorname{div} \mathbf{g} \in L^{\infty}\left(\mathbb{R}^{n}\right)$, so, in particular, it is valid for all $\mathbf{g} \in C_{c}^{1}\left(\mathbb{R}^{n}, \mathbb{R}^{n}\right)$, which implies that the function

$$
\operatorname{deg}(\mathbf{u}, U, \cdot)-\sum_{k \in \mathbb{N}} \operatorname{deg}\left(\mathbf{u}, B_{k}, \cdot\right)
$$

is constant a.e., but with (4.12) we obtain that, in fact,

$$
\operatorname{deg}(\mathbf{u}, U, \cdot)=\sum_{k \in \mathbb{N}} \operatorname{deg}\left(\mathbf{u}, B_{k}, \cdot\right) \geq 0 \quad \text { a.e. },
$$

which proves $c$ ).

Part d). Let $U_{1}, U_{2} \in \mathcal{U}_{\mathbf{u}}$ satisfy $U_{1} \subset \subset U_{2}$. By Lemma 2.22 , we have that $U_{2} \backslash \bar{U}_{1} \in \mathcal{U}_{\mathbf{u}}$, so by $c$ ) we obtain that $\operatorname{deg}\left(\mathbf{u}, U_{2} \backslash \bar{U}_{1}, \cdot\right) \geq 0$ a.e. Thanks to the additivity of the Brouwer degree (see, e.g., [21, Th. 3.1 (d2)]), for all $\mathbf{y} \in \mathbb{R}^{n} \backslash \mathbf{u}\left(\partial U_{1} \cup \partial U_{2}\right)$,

$$
\operatorname{deg}\left(\mathbf{u}, U_{2}, \mathbf{y}\right)=\operatorname{deg}\left(\mathbf{u}, U_{2} \backslash \bar{U}_{1}, \mathbf{y}\right)+\operatorname{deg}\left(\mathbf{u}, U_{1}, \mathbf{y}\right) .
$$

We thus obtain that $\operatorname{deg}\left(\mathbf{u}, U_{1}, \cdot\right) \leq \operatorname{deg}\left(\mathbf{u}, U_{2}, \cdot\right)$ a.e. In particular, $\operatorname{im}_{\mathrm{T}}\left(\mathbf{u}, U_{1}\right) \subset \operatorname{im}_{\mathrm{T}}\left(\mathbf{u}, U_{2}\right) \cup \mathbf{u}\left(\partial U_{2}\right)$. As $\mathcal{L}^{n}\left(\mathbf{u}\left(\partial U_{2}\right)\right)=0$ and $\operatorname{im}_{\mathrm{T}}\left(\mathbf{u}, U_{1}\right)$ is open, given $\mathbf{y}_{0} \in \operatorname{im}_{\mathrm{T}}\left(\mathbf{u}, U_{1}\right)$ there exists a sequence $\left\{\mathbf{y}_{j}\right\}_{j \in \mathbb{N}}$ in $\mathbb{R}^{n} \backslash \mathbf{u}\left(\partial U_{2}\right)$ tending to $\mathbf{y}_{0}$. Therefore, $\mathbf{y}_{j} \in \operatorname{im}_{\mathrm{T}}\left(\mathbf{u}, U_{2}\right)$ for all $j \in \mathbb{N}$. This shows that $\operatorname{im}_{\mathrm{T}}\left(\mathbf{u}, U_{1}\right) \subset \overline{\operatorname{im}_{\mathrm{T}}\left(\mathbf{u}, U_{2}\right)}$ and, consequently, $\overline{\mathrm{im}_{\mathrm{T}}\left(\mathbf{u}, U_{1}\right)} \subset \overline{\mathrm{im}_{\mathrm{T}}\left(\mathbf{u}, U_{2}\right)}$, which concludes the proof of $\left.d\right)$.

Part e). Define

$$
\begin{aligned}
U_{+} & :=\left\{\mathbf{x} \in U \cap \Omega_{0}: \mathbf{u}^{*} \text { has a regular approximate differential at } \mathbf{x} \text { with } \operatorname{det} \nabla \mathbf{u}^{*}(\mathbf{x})>0\right\}, \\
U_{0} & :=\left\{\mathbf{x} \in U \cap \Omega_{0}: \mathbf{u}^{*} \text { has a regular approximate differential at } \mathbf{x} w i t h \operatorname{det} \nabla \mathbf{u}^{*}(\mathbf{x})=0\right\},
\end{aligned}
$$

where $\mathbf{u}^{*}$ is the precise representative of Definition 2.2. Proposition 2.6, Lemma 3.2 and the non-negativity of the degree show that $U_{+} \cup U_{0}=U$ a.e. Lemma 3.2 also implies that for all $\mathbf{x} \in U_{+}$there exists $r>0$ 
such that $B(\mathbf{x}, r) \in \mathcal{U}_{\mathbf{u}}, B(\mathbf{x}, r) \subset \subset U$, and $\mathbf{u}^{*}(\mathbf{x}) \in \operatorname{im}_{\mathrm{T}}(\mathbf{u}, B(\mathbf{x}, r))$; then, by part $\left.d\right), \mathbf{u}^{*}(\mathbf{x}) \in \overline{\operatorname{im}_{\mathrm{T}}(\mathbf{u}, U)}$. On the other hand, Proposition 2.7 shows that $\mathcal{L}^{n}\left(\operatorname{im}_{\mathrm{G}}\left(\mathbf{u}, U_{0}\right)\right)=0$. Altogether, $\operatorname{im}_{\mathrm{G}}(\mathbf{u}, U) \subset \overline{\operatorname{im}_{\mathrm{T}}(\mathbf{u}, U)}$ a.e. By Proposition 2.12, each $\mathbf{y}_{0} \in \operatorname{im}_{\mathrm{G}}(\mathbf{u}, U)$ satisfies $D\left(\overline{\operatorname{im}_{\mathrm{T}}(\mathbf{u}, U)}, \mathbf{y}_{0}\right)=1$; since $\overline{\operatorname{im}_{\mathrm{T}}(\mathbf{u}, U)}$ is closed, we must have that $\mathbf{y}_{0} \in \overline{\operatorname{im}_{\mathrm{T}}(\mathbf{u}, U)}$. Therefore, $\operatorname{im}_{\mathrm{G}}(\mathbf{u}, U) \subset \overline{\operatorname{im}_{\mathrm{T}}(\mathbf{u}, U)}$ and $\mathbf{u} \in L^{\infty}\left(U, \mathbb{R}^{n}\right)$.

Part b). Thanks to parts a) and $e$ ), we are in the assumptions of De Lellis \& Ghiraldin [20, Th. 1.2], and can conclude that Det $D \mathbf{u}=(\operatorname{det} D \mathbf{u}) \mathcal{L}^{n}+\mu^{s}$ for some measure $\mu^{s}$ that is singular with respect to the Lebesgue measure. As a consequence of this decomposition, $\operatorname{det} D \mathbf{u} \in L_{\mathrm{loc}}^{1}(\Omega)$ and $\operatorname{det} D \mathbf{u} \geq 0$ a.e.

Part f). Assume Det $D \mathbf{u}=\operatorname{det} D \mathbf{u}$, take $U \in \mathcal{U}_{\mathbf{u}}$ and let $\mathcal{F}$ be a family of balls as in the statement. Let $\mathcal{F}^{\prime}$ be the set of $\bar{B}$ such that $B \in \mathcal{F} \cap \mathcal{U}_{\mathbf{u}}$. Let $A$ be the set of points $\mathbf{x} \in U$ for which (4.6) is satisfied. By Besicovitch's covering theorem, there exists a countable family $\left\{B_{k}\right\}_{k \in \mathbb{N}} \subset \mathcal{F} \cap \mathcal{U}_{\mathbf{u}}$ of balls with disjoint closures such that $A=\bigcup_{k \in \mathbb{N}} B_{k}$ a.e. On the other hand, by hypothesis, $U=A$ a.e. Therefore,

$$
U=\bigcup_{k \in \mathbb{N}} B_{k} \quad \text { a.e. }
$$

From this point, we can apply verbatim the reasoning of part $c$ ) of the proof, having in mind that Det $D \mathbf{u}$ and $\Lambda_{\mathbf{g}}$ are absolutely continuous with respect to the Lebesgue measure. We conclude that $\operatorname{deg}(\mathbf{u}, U, \cdot)=$ $\sum_{k \in \mathbb{N}} \operatorname{deg}\left(\mathbf{u}, B_{k}, \cdot\right)$ a.e., which finishes the proof.

In order to prove that maps with nonnegative degree and satisfying $\operatorname{Det} D \mathbf{u}=\operatorname{det} D \mathbf{u}$ necessarily have zero surface energy, we need to establish first that these two conditions imply the local invertibility of the deformation.

Definition 4.4. Let $\mathbf{u} \in W^{1, p}\left(\Omega, \mathbb{R}^{n}\right), p>n-1$. We define $\mathcal{U}_{\mathbf{u}}^{\text {in }}$ as the class of $U \in \mathcal{U}_{\mathbf{u}}$ such that $\mathbf{u}$ is one-to-one a.e. in $U$. We denote by $\Omega_{\text {in }}$ the set $\bigcup \mathcal{U}_{\mathbf{u}}^{\text {in }}$.

Thus, $\Omega_{\text {in }}$ consists of the sets of points around which $\mathbf{u}$ is locally a.e. invertible: $\mathbf{x} \in \Omega_{\text {in }}$ if and only if there exists $r>0$ such that $\mathbf{u}$ is one-to-one a.e. in $B(\mathbf{x}, r)$. The set $\Omega_{\mathrm{in}}$ does not depend on the particular representative of $\mathbf{u}$. Indeed, if $\mathbf{u}_{1}=\mathbf{u}_{2}$ a.e. and $\mathbf{x} \in U_{1}$ with $U_{1} \in \mathcal{U}_{\mathbf{u}_{1}}$ satisfies that $\mathbf{u}_{1}$ is one-to-one a.e. in $U_{1}$ then, by Lemma 2.20, there exists $U_{2} \in \mathcal{U}_{\mathbf{u}_{2}}$ with $\mathbf{x} \in U_{2}$ and $U_{2} \subset U_{1}$, so $\mathbf{u}_{2}$ is one-to-one a.e. in $U_{2}$.

Proposition 4.5. Let $p>n-1$ and $\mathbf{u} \in W^{1, p}\left(\Omega, \mathbb{R}^{n}\right)$. Assume that $(\operatorname{adj} D \mathbf{u}) \mathbf{u} \in L_{\text {loc }}^{1}\left(\Omega, \mathbb{R}^{n}\right)$ and that $\operatorname{deg}(\mathbf{u}, B(\mathbf{x}, r), \cdot) \geq 0$ a.e. for all $\mathbf{x} \in \Omega$ and $r>0$ for which $B(\mathbf{x}, r) \in \mathcal{U}_{\mathbf{u}}$.

a) Suppose that $U \in \mathcal{U}_{\mathbf{u}}$ and that $\mathbf{u}^{*}$ has a regular approximate differential at two points $\mathbf{x}_{1}, \mathbf{x}_{2} \in U$. Suppose, further, that $\mathbf{x}_{1} \in \Omega_{0}$, $\operatorname{det} \nabla \mathbf{u}\left(\mathbf{x}_{2}\right) \neq 0, \mathbf{u}\left(\mathbf{x}_{1}\right)=\mathbf{u}\left(\mathbf{x}_{2}\right):=\mathbf{y}_{0}$, and

$$
D\left(\left\{\mathbf{y} \in \mathbb{R}^{n} \backslash \mathbf{u}(\partial U): \operatorname{deg}(\mathbf{u}, U, \mathbf{y})=1\right\}, \mathbf{y}_{0}\right)=1 .
$$

Then $\mathbf{x}_{1}=\mathbf{x}_{2}$.

b) Suppose that $U_{1}, U_{2} \in \mathcal{U}_{\mathbf{u}}, U_{1} \subset U_{2}$ and $\operatorname{deg}\left(\mathbf{u}, U_{2}, \cdot\right)=1$ a.e. $i n \operatorname{im}_{\mathrm{T}}\left(\mathbf{u}, U_{1}\right)$. Assume further that $\operatorname{det} \nabla \mathbf{u}(\mathbf{x}) \neq 0$ for a.e. $\mathbf{x} \in U_{2}$. Then $\mathbf{u}$ is one-to-one a.e. in $U_{1}$ and $\mathbf{u}(\mathbf{x}) \notin \operatorname{im}_{\mathrm{G}}\left(\mathbf{u}, U_{1}\right)$ for a.e. $\mathbf{x} \in U_{2} \backslash U_{1}$.

c) Suppose that $U_{1}, U_{2} \in \mathcal{U}_{\mathbf{u}}, U_{1} \subset \subset U_{2}, \partial U_{1}$ and $\partial U_{2}$ are connected, $\mathbf{u}\left(\partial U_{1}\right) \cap \mathbf{u}\left(\partial U_{2}\right)=\varnothing$ and $\operatorname{deg}\left(\mathbf{u}, U_{2}, \cdot\right)=$ 1 on $\mathbf{u}\left(\partial U_{1}\right)$. Then $\operatorname{deg}\left(\mathbf{u}, U_{2}, \mathbf{y}\right)=1$ for every $\mathbf{y} \in \operatorname{im}_{\mathrm{T}}\left(\mathbf{u}, U_{1}\right)$.

d) $\Omega_{\text {in }}$ is open and $\left\{\mathbf{x} \in \Omega_{d}: \operatorname{det} \nabla \mathbf{u}(\mathbf{x}) \neq 0\right\} \subset \Omega_{\text {in }}$ a.e.

Proof. Part a). Suppose, for a contradiction, that $\mathbf{x}_{1} \neq \mathbf{x}_{2}$. By Lemma 3.2, for $i \in\{1,2\}$ there exists $r_{i}>0$ such that the balls $B_{i}:=B\left(\mathbf{x}_{i}, r_{i}\right)$ satisfy $B_{i} \in \mathcal{U}_{\mathbf{u}}, \mathbf{y}_{0} \notin \mathbf{u}\left(\partial B_{i}\right), \bar{B}_{1} \cap \bar{B}_{2}=\varnothing, \bar{B}_{1} \cup \bar{B}_{2} \subset U$ and

$$
\operatorname{deg}\left(\mathbf{u}, B_{i}, \cdot\right)=1 \quad \text { in } C_{i}
$$


where $C_{i}$ is the connected component of $\mathbb{R}^{n} \backslash \mathbf{u}\left(\partial B_{i}\right)$ containing $\mathbf{y}_{0}$. Thus,

$$
\operatorname{deg}\left(\mathbf{u}, B_{1}, \cdot\right)=\operatorname{deg}\left(\mathbf{u}, B_{2}, \cdot\right)=1 \quad \text { in } C_{1} \cap C_{2} .
$$

By Lemma 2.22 and Proposition $4.3 d$ ),

$$
\operatorname{deg}\left(\mathbf{u}, B_{1} \cup B_{2}, \cdot\right) \leq \operatorname{deg}(\mathbf{u}, U, \cdot) \quad \text { a.e. }
$$

But, by the additivity of the degree (see, e.g., [21, Th. 3.1 (d2)]),

$$
\operatorname{deg}\left(\mathbf{u}, B_{1} \cup B_{2}, \cdot\right)=\operatorname{deg}\left(\mathbf{u}, B_{1}, \cdot\right)+\operatorname{deg}\left(\mathbf{u}, B_{2}, \cdot\right) \quad \text { in } \mathbb{R}^{n} \backslash\left(\mathbf{u}\left(\partial B_{1}\right) \cup \mathbf{u}\left(\partial B_{2}\right)\right) .
$$

Thus, for a.e. $\mathbf{y} \in C_{1} \cap C_{2}$, owing to (4.15), (4.16) and (4.17),

$$
2=\operatorname{deg}\left(\mathbf{u}, B_{1}, \mathbf{y}\right)+\operatorname{deg}\left(\mathbf{u}, B_{2}, \mathbf{y}\right)=\operatorname{deg}\left(\mathbf{u}, B_{1} \cup B_{2}, \mathbf{y}\right) \leq \operatorname{deg}(\mathbf{u}, U, \mathbf{y}) .
$$

In particular, since $C_{1} \cap C_{2}$ is an open neighbourhood of $\mathbf{y}_{0}$,

$$
D\left(\left\{\mathbf{y} \in \mathbb{R}^{n} \backslash \mathbf{u}(\partial U): \operatorname{deg}(\mathbf{u}, U, \mathbf{y}) \geq 2\right\}, \mathbf{y}_{0}\right)=1,
$$

which contradicts (4.14).

Part b). By Remark 2.3, Proposition 2.6, the fact that $\mathcal{L}^{n}\left(\Omega \backslash \Omega_{0}\right)=0$ and Lemma 2.8, it suffices to prove that if $\mathbf{u}^{*}$ has a regular approximate differential at two points $\mathbf{x}_{1} \in U_{1} \cap \Omega_{0}$ and $\mathbf{x}_{2} \in U_{2}$ with $\operatorname{det} \nabla \mathbf{u}\left(\mathbf{x}_{2}\right) \neq 0$ and $\mathbf{u}^{*}\left(\mathbf{x}_{1}\right)=\mathbf{u}^{*}\left(\mathbf{x}_{2}\right)$ then $\mathbf{x}_{1}=\mathbf{x}_{2}$. Call $\mathbf{y}_{0}:=\mathbf{u}^{*}\left(\mathbf{x}_{1}\right)$. By Proposition 2.12, $D\left(\operatorname{im}_{\mathrm{G}}\left(\mathbf{u}, U_{1}\right), \mathbf{y}_{0}\right)=1$, so thanks to Proposition $4.3 e)$ we obtain $D\left(\operatorname{im}_{\mathrm{T}}\left(\mathbf{u}, U_{1}\right), \mathbf{y}_{0}\right)=1$. Our assumption indicates that

$$
\operatorname{im}_{\mathrm{T}}\left(\mathbf{u}, U_{1}\right) \subset\left\{\mathbf{y} \in \mathbb{R}^{n} \backslash \mathbf{u}\left(\partial U_{2}\right): \operatorname{deg}\left(\mathbf{u}, U_{2}, \mathbf{y}\right)=1\right\} \quad \text { a.e., }
$$

and, hence,

$$
D\left(\left\{\mathbf{y} \in \mathbb{R}^{n} \backslash \mathbf{u}\left(\partial U_{2}\right): \operatorname{deg}\left(\mathbf{u}, U_{2}, \mathbf{y}\right)=1\right\}, \mathbf{y}_{0}\right)=1 .
$$

Part a) then concludes that $\mathbf{x}_{1}=\mathbf{x}_{2}$.

Part c). The set $\mathbf{u}\left(\partial U_{1}\right)$ is connected and contained in $\mathbb{R}^{n} \backslash \mathbf{u}\left(\partial U_{2}\right)$; let $C$ be the connected component of $\mathbb{R}^{n} \backslash \mathbf{u}\left(\partial U_{2}\right)$ containing $\mathbf{u}\left(\partial U_{1}\right)$. Since $\operatorname{deg}\left(\mathbf{u}, U_{2}, \cdot\right)=1$ in $C$, our aim is to show that $\operatorname{im}_{\mathrm{T}}\left(\mathbf{u}, U_{1}\right) \subset C$. As $\mathbf{u}\left(\partial U_{2}\right)$ is connected, we have that $\mathbb{R}^{n} \backslash C$ is connected (see, e.g., [12, Ex. I.11.4 b)]) and contained in $\mathbb{R}^{n} \backslash \mathbf{u}\left(\partial U_{1}\right)$, so $\mathbb{R}^{n} \backslash C$, being unbounded, must be contained in the unbounded component $D$ of $\mathbb{R}^{n} \backslash \mathbf{u}\left(\partial U_{1}\right)$. As $\operatorname{deg}\left(\mathbf{u}, U_{1}, \cdot\right)=0$ in $D$, we have that $\operatorname{im}_{\mathrm{T}}\left(\mathbf{u}, U_{1}\right) \subset \mathbb{R}^{n} \backslash D$. As $\mathbb{R}^{n} \backslash D \subset C$, the proof of $\left.c\right)$ is done.

Part d). It is clear that $\Omega_{\text {in }}$ is open, as a union of open sets. In order to prove that $\mathcal{L}^{n}\left(\left\{\mathbf{x} \in \Omega_{d}\right.\right.$ : $\left.\operatorname{det} \nabla \mathbf{u}(\mathbf{x}) \neq 0\} \backslash \Omega_{\text {in }}\right)=0$, by Proposition 2.6 and Proposition $\left.4.3 b\right)$ it suffices to show that $\mathbf{x}_{0} \in \Omega_{\text {in }}$ for every $\mathbf{x}_{0} \in \Omega_{d}$ at which det $\nabla \mathbf{u}\left(\mathbf{x}_{0}\right)>0$ and $\mathbf{u}^{*}$ has a regular approximate differential. By Lemma 3.2, there exists $r>0$ such that the ball $B:=B\left(\mathbf{x}_{0}, r\right)$ satisfies $B \in \mathcal{U}_{\mathbf{u}}, \mathbf{u}^{*}\left(\mathbf{x}_{0}\right) \notin \mathbf{u}(\partial B)$ and

$$
\operatorname{deg}(\mathbf{u}, B, \mathbf{y})=1 \quad \text { for all } \mathbf{y} \in C_{0}
$$

where $C_{0}$ is the connected component of $\mathbb{R}^{n} \backslash \mathbf{u}(\partial B)$ containing $\mathbf{u}\left(\mathbf{x}_{0}\right)$. Moreover, there also exists $r^{\prime}<r$ such that the ball $B^{\prime}:=B\left(\mathbf{x}_{0}, r^{\prime}\right)$ satisfies $B^{\prime} \in \mathcal{U}_{\mathbf{u}}$ and $\mathbf{u}\left(\partial B^{\prime}\right) \subset C_{0}$. Part $\left.c\right)$ then shows that $\operatorname{deg}(\mathbf{u}, B, \cdot)=1$ in $\operatorname{im}_{\mathrm{T}}\left(\mathbf{u}, B^{\prime}\right)$, while Part b) yields that $\mathbf{u}$ is one-to-one a.e. in $B^{\prime}$, so $\mathbf{x}_{0} \in \Omega_{\text {in }}$ and $d$ ) is proved.

We are now in a position to conclude our characterization.

Theorem 4.6. Let $\mathbf{u} \in W^{1, p}\left(\Omega, \mathbb{R}^{n}\right), p>n-1$, satisfy $\operatorname{det} D \mathbf{u} \in L_{\text {loc }}^{1}(\Omega),(\operatorname{adj} D \mathbf{u}) \mathbf{u} \in L_{\text {loc }}^{1}\left(\Omega, \mathbb{R}^{n}\right)$, $\operatorname{det} D \mathbf{u}(\mathbf{x}) \neq 0$ a.e. $\mathbf{x} \in \Omega$, $\operatorname{Det} D \mathbf{u}=\operatorname{det} D \mathbf{u}$, and $\operatorname{deg}(\mathbf{u}, B(\mathbf{x}, r), \cdot) \geq 0$ a.e. for all $\mathbf{x} \in \Omega$ and $r>0$ for which $B(\mathbf{x}, r) \in \mathcal{U}_{\mathbf{u}}$. Then $\mathcal{E}(\mathbf{u})=0$ and $\operatorname{det} D \mathbf{u}>0$ a.e. 
Proof. Property det $D \mathbf{u}>0$ a.e. is an immediate consequence of Proposition $4.3 \mathrm{~b}$ ).

We show that, for every $U \in \mathcal{U}_{\mathbf{u}}^{\text {in }}$,

$$
\operatorname{deg}(\mathbf{u}, U, \cdot)=\mathcal{N}_{U}=\chi_{\operatorname{im}_{\mathrm{G}}(\mathbf{u}, U)}=\chi_{\mathrm{im}_{\mathrm{T}}(\mathbf{u}, U)} \quad \text { a.e. }
$$

By Proposition $4.3 e$ ),

$$
\operatorname{im}_{\mathrm{G}}(\mathbf{u}, U) \subset \operatorname{im}_{\mathrm{T}}(\mathbf{u}, U) \quad \text { a.e. }
$$

As, by Proposition $4.3 c), \operatorname{deg}(\mathbf{u}, U, \cdot) \geq 0$ a.e., we have, using also Proposition 3.3,

$$
\mathcal{L}^{n}\left(\operatorname{im}_{\mathrm{T}}(\mathbf{u}, U)\right) \leq \int_{\mathbb{R}^{n}} \operatorname{deg}(\mathbf{u}, U, \mathbf{y}) \mathrm{d} \mathbf{y}=\frac{1}{n} \int_{\partial U} \mathbf{u}(\mathbf{x}) \cdot(\operatorname{cof} D \mathbf{u}(\mathbf{x}) \boldsymbol{\nu}(\mathbf{x})) \mathrm{d} \mathcal{H}^{n-1}(\mathbf{x}),
$$

where $\boldsymbol{\nu}$ is the unit outward normal to $U$.

Let $\left\{\phi_{\delta}\right\}_{\delta>0}$ be the family of Proposition 2.23. Then

$$
\frac{1}{n} \int_{\partial U} \mathbf{u}(\mathbf{x}) \cdot(\operatorname{cof} D \mathbf{u}(\mathbf{x}) \boldsymbol{\nu}(\mathbf{x})) \mathrm{d} \mathcal{H}^{n-1}(\mathbf{x})=-\frac{1}{n} \lim _{\delta \searrow 0} \int_{\Omega} \mathbf{u}(\mathbf{x}) \cdot\left(\operatorname{cof} D \mathbf{u}(\mathbf{x}) D \phi_{\delta}(\mathbf{x})\right) \mathrm{d} \mathbf{x}=\lim _{\delta \searrow 0}\left\langle\operatorname{Det} D \mathbf{u}, \phi_{\delta}\right\rangle .
$$

Using the assumption Det $D \mathbf{u}=\operatorname{det} D \mathbf{u}$ and dominated convergence we obtain

$$
\lim _{\delta \searrow 0}\left\langle\operatorname{Det} D \mathbf{u}, \phi_{\delta}\right\rangle=\lim _{\delta \searrow 0} \int_{\Omega} \phi_{\delta}(\mathbf{x}) \operatorname{det} D \mathbf{u}(\mathbf{x}) \mathrm{d} \mathbf{x}=\int_{U} \operatorname{det} D \mathbf{u}(\mathbf{x}) \mathrm{d} \mathbf{x} .
$$

By Proposition 2.7 and the fact that $\mathbf{u}$ is one-to-one a.e. in $U$, we obtain

$$
\int_{U} \operatorname{det} D \mathbf{u}(\mathbf{x}) \mathrm{d} \mathbf{x}=\int_{\mathbb{R}^{n}} \mathcal{N}_{U}(\mathbf{y}) \mathrm{d} \mathbf{y}=\mathcal{L}^{n}\left(\operatorname{im}_{G}(\mathbf{u}, U)\right) .
$$

We recapitulate the conclusion of relations (4.20), (4.21), (4.22), (4.23) and (4.19):

$$
\mathcal{L}^{n}\left(\operatorname{im}_{\mathrm{T}}(\mathbf{u}, U)\right) \leq \int_{\mathbb{R}^{n}} \operatorname{deg}(\mathbf{u}, U, \mathbf{y}) \mathrm{d} \mathbf{y}=\int_{\mathbb{R}^{n}} \mathcal{N}_{U}(\mathbf{y}) \mathrm{d} \mathbf{y}=\mathcal{L}^{n}\left(\operatorname{im}_{\mathrm{G}}(\mathbf{u}, U)\right) \leq \mathcal{L}^{n}\left(\operatorname{im}_{\mathrm{T}}(\mathbf{u}, U)\right) .
$$

This chain of inequalities proves the validity of (4.18).

Now fix $\phi \in C_{c}^{1}(\Omega)$ and $\mathbf{g} \in C_{c}^{1}\left(\mathbb{R}^{n}, \mathbb{R}^{n}\right)$, and consider the functions $\psi_{t}$ and $\varphi_{t}$ of (4.5). For each $0<t<1$ define $h_{t}: \operatorname{spt} \phi \rightarrow \mathbb{R}$ as

$$
h_{t}(\mathbf{z}):=\int_{0}^{t} \psi_{t}^{\prime}(r) \int_{\mathbb{R}^{n}} \operatorname{div} \mathbf{g}(\mathbf{y}) \operatorname{deg}(\mathbf{u}, B(\mathbf{z}, r), \mathbf{y}) \mathrm{d} \mathbf{y} \mathrm{d} r .
$$

By Lemma 4.2,

$$
\int_{\Omega} \mathbf{g}(\mathbf{u}(\mathbf{x})) \cdot(\operatorname{cof} D \mathbf{u}(\mathbf{x}) D \phi(\mathbf{x})) \mathrm{d} \mathbf{x}=\lim _{t \searrow 0} \int_{\operatorname{spt} \phi} \phi(\mathbf{z}) h_{t}(\mathbf{z}) \mathrm{d} \mathbf{z} .
$$

Fix $\mathbf{z} \in \operatorname{spt} \phi$ and $r>0$ such that $B(\mathbf{z}, r) \in \mathcal{U}_{\mathbf{u}}$. Thanks to (4.18) and Proposition $4.5 d$ ), we can apply Proposition $4.3 \mathrm{f}$ ) to the family $\mathcal{F}$ of balls $B \in \mathcal{U}_{\mathbf{u}}$ such that $B \subset B(\mathbf{z}, r)$ and

$$
\operatorname{deg}(\mathbf{u}, B, \cdot)=\mathcal{N}_{B}=\chi_{\mathrm{im}_{\mathrm{G}}(\mathbf{u}, B)} \quad \text { a.e. }
$$

Thus, there exists a disjoint sequence $\left\{B_{k}\right\}_{k \in \mathbb{N}}$ in $\mathcal{U}_{\mathbf{u}}$ such that

$$
B(\mathbf{z}, r)=\bigcup_{k \in \mathbb{N}} B_{k} \quad \text { a.e., } \quad \operatorname{deg}(\mathbf{u}, B(\mathbf{z}, r), \cdot)=\sum_{k \in \mathbb{N}} \operatorname{deg}\left(\mathbf{u}, B_{k}, \cdot\right) \quad \text { a.e. }
$$

and, for all $k \in \mathbb{N}$,

$$
\operatorname{deg}\left(\mathbf{u}, B_{k}, \cdot\right)=\mathcal{N}_{B_{k}}=\chi_{\mathrm{im}_{\mathrm{G}}\left(\mathbf{u}, B_{k}\right)} \quad \text { a.e. }
$$


Therefore, by dominated convergence, (4.26), (4.27) and Proposition 2.7,

$$
\begin{aligned}
\int_{\mathbb{R}^{n}} \operatorname{div} \mathbf{g}(\mathbf{y}) \operatorname{deg}(\mathbf{u}, B(\mathbf{z}, r), \mathbf{y}) \mathrm{d} \mathbf{y} & =\sum_{k \in \mathbb{N}} \int_{\mathbb{R}^{n}} \operatorname{div} \mathbf{g}(\mathbf{y}) \operatorname{deg}\left(\mathbf{u}, B_{k}, \mathbf{y}\right) \mathrm{d} \mathbf{y}=\sum_{k \in \mathbb{N}} \int_{\operatorname{im}_{\mathrm{G}}\left(\mathbf{u}, B_{k}\right)} \operatorname{div} \mathbf{g}(\mathbf{y}) \mathrm{d} \mathbf{y} \\
& =\sum_{k \in \mathbb{N}} \int_{B_{k}} \operatorname{div} \mathbf{g}(\mathbf{u}(\mathbf{x})) \operatorname{det} D \mathbf{u}(\mathbf{x}) \mathrm{d} \mathbf{x}=\int_{B(\mathbf{z}, r)} \operatorname{div} \mathbf{g}(\mathbf{u}(\mathbf{x})) \operatorname{det} D \mathbf{u}(\mathbf{x}) \mathrm{d} \mathbf{x} .
\end{aligned}
$$

This and (4.24) imply that

$$
h_{t}(\mathbf{z})=\int_{0}^{t} \psi_{t}^{\prime}(r) \int_{B(\mathbf{z}, r)} \operatorname{div} \mathbf{g}(\mathbf{u}(\mathbf{x})) \operatorname{det} D \mathbf{u}(\mathbf{x}) \mathrm{d} \mathbf{x} \mathrm{d} r .
$$

Noticing that, thanks to the fundamental theorem of Calculus and the coarea formula,

$$
\frac{\mathrm{d}}{\mathrm{d} r} \int_{B(\mathbf{z}, r)} \operatorname{div} \mathbf{g}(\mathbf{u}(\mathbf{x})) \operatorname{det} D \mathbf{u}(\mathbf{x}) \mathrm{d} \mathbf{x} \mathrm{d} r=\int_{\partial B(\mathbf{z}, r)} \operatorname{div} \mathbf{g}(\mathbf{u}(\mathbf{x})) \operatorname{det} D \mathbf{u}(\mathbf{x}) \mathrm{d} \mathcal{H}^{n-1}(\mathbf{x}),
$$

an integration by parts and again the coarea formula yield

$$
\begin{aligned}
-h_{t}(\mathbf{z}) & =\int_{0}^{t} \psi_{t}(r) \int_{\partial B(\mathbf{z}, r)} \operatorname{div} \mathbf{g}(\mathbf{u}(\mathbf{x})) \operatorname{det} D \mathbf{u}(\mathbf{x}) \mathrm{d} \mathcal{H}^{n-1}(\mathbf{x}) \mathrm{d} r \\
& =\int_{B(\mathbf{z}, t)} \varphi_{t}(\mathbf{z}-\mathbf{x}) \operatorname{div} \mathbf{g}(\mathbf{u}(\mathbf{x})) \operatorname{det} D \mathbf{u}(\mathbf{x}) \mathrm{d} \mathbf{x}=\left(\varphi_{t} \star((\operatorname{div} \mathbf{g} \circ \mathbf{u})(\operatorname{det} D \mathbf{u}))\right)(\mathbf{z}) .
\end{aligned}
$$

Hence, we can apply a standard property of convolutions and obtain that

$$
\lim _{t \searrow 0} \int_{\operatorname{spt} \phi} \phi(\mathbf{z}) h_{t}(\mathbf{z}) \mathrm{d} \mathbf{z}=-\int_{\operatorname{spt} \phi} \phi(\mathbf{z}) \operatorname{div} \mathbf{g}(\mathbf{u}(\mathbf{z})) \operatorname{det} D \mathbf{u}(\mathbf{z}) \mathrm{d} \mathbf{z} .
$$

Equalities (4.25) and (4.28) show that $\mathcal{E}(\mathbf{u}, \phi \mathbf{g})=0$, i.e., equality $(2.4)$ holds, and, consequently, $\mathcal{E}(\mathbf{u})=0$. This concludes the proof.

Note that, thanks to Proposition 4.3, assumptions $\operatorname{det} D \mathbf{u} \in L_{\text {loc }}^{1}(\Omega)$ and $\operatorname{Det} D \mathbf{u}=\operatorname{det} D \mathbf{u}$ in Theorem 4.6 are equivalent to saying that the measure Det $D \mathbf{u}$ is absolutely continuous with respect to $\mathcal{L}^{n}$. For ease of reference, Theorems 1.1, 4.1 and 4.6, and Proposition $4.5 \mathrm{~d}$ ) are summarized in the following corollary.

Corollary 4.7. Let $\mathbf{u} \in W^{1, p}\left(\Omega, \mathbb{R}^{n}\right), p>n-1$, $\operatorname{det} D \mathbf{u} \in L_{\mathrm{loc}}^{1}(\Omega)$. Then the following conditions are equivalent:

a) $\mathcal{E}(\mathbf{u})=0$ and $\operatorname{det} D \mathbf{u}>0$ a.e.

b) $(\operatorname{adj} D \mathbf{u}) \mathbf{u} \in L_{\text {loc }}^{1}\left(\Omega, \mathbb{R}^{n}\right)$, $\operatorname{det} D \mathbf{u}(\mathbf{x}) \neq 0$ for a.e. $\mathbf{x} \in \Omega$, Det $D \mathbf{u}=\operatorname{det} D \mathbf{u}$, and $\operatorname{deg}(\mathbf{u}, B(\mathbf{x}, r), \cdot) \geq 0$ a.e. for all $\mathbf{x} \in \Omega$ and $r>0$ for which $B(\mathbf{x}, r) \in \mathcal{U}_{\mathbf{u}}$.

Moreover, in either case we have that $\mathbf{u} \in L_{\mathrm{loc}}^{\infty}\left(\Omega, \mathbb{R}^{n}\right)$, for all $U \in \mathcal{U}_{\mathbf{u}}$ formula (4.1) holds, and for a.e. $\mathbf{x} \in \Omega$ there exists $r_{0}>0$ such that $\mathbf{u}$ is one-to-one a.e. in $B\left(\mathbf{x}, r_{0}\right)$.

Motivated by Corollary 4.7, we introduce the functional class that is the object of our work.

Definition 4.8. For each $p>n-1$, we define $\mathcal{A}_{p}$ as the set of $\mathbf{u} \in W^{1, p}\left(\Omega, \mathbb{R}^{n}\right)$ such that $\operatorname{det} D \mathbf{u} \in L_{\text {loc }}^{1}(\Omega)$, $\operatorname{det} D \mathbf{u}>0$ a.e. and $\mathcal{E}(\mathbf{u})=0$.

The class $\mathcal{A}_{p}$ is not really new since, except for the requirement of the positivity of the determinant, it was introduced by Müller [59] without giving it a name. It was also used in Giaquinta, Modica \& Souček [38, Def. 3.2.1.3 and Prop. 3.2.4.1].

As mentioned in the introduction, if $p>n-1, \mathbf{u} \in W^{1, p}\left(\Omega, \mathbb{R}^{n}\right)$, cof $D \mathbf{u} \in L^{\frac{n}{n-1}}\left(\Omega, \mathbb{R}^{n \times n}\right)$ and $\operatorname{det} D \mathbf{u}>0$ a.e. then $\mathbf{u}$ belongs to the functional space we have just defined (it follows from Müller, Qi \& Yan [63, Th. $3.2])$.

A more accurate notation for $\mathcal{A}_{p}$ would be $\mathcal{A}_{p}(\Omega)$, in order to stress the dependence on $\Omega$. However, since the domain $\Omega$ is fixed throughout the paper, we prefer to use the notation $\mathcal{A}_{p}$. 


\section{$5 \quad$ Fine properties of deformations in $\mathcal{A}_{p}$}

In this section we show some fine properties of the deformations in the class $\mathcal{A}_{p}$ and of their local inverses.

\subsection{Condition INV and Sobolev regularity of the inverse}

We begin by noting that equality (4.1) implies a sort of openness property for $\mathbf{u}$ : for every $U \in \mathcal{U}_{\mathbf{u}}$,

$$
\operatorname{im}_{\mathrm{T}}(\mathbf{u}, U)=\operatorname{im}_{\mathrm{G}}(\mathbf{u}, U) \text { a.e. }
$$

Next, we show that in our class $\mathcal{A}_{p}$, the a.e. invertibility of a map in a subdomain is equivalent to condition INV (Definition 3.5). Müller \& Spector [61] showed that, under the assumption $\operatorname{det} D \mathbf{u}>0$ a.e., INV implies invertibility a.e, but the converse is false in general (see, e.g., [61, Sect. 11], [62, Sect. 5] or [44, Sect. 7]).

Lemma 5.1. Let $\mathbf{u} \in \mathcal{A}_{p}$.

a) Given $U \in \mathcal{U}_{\mathbf{u}}$, one has that $\left.\mathbf{u}\right|_{U}$ satisfies INV if and only if $\mathbf{u}$ is one-to-one a.e. in $U$.

b) Given $U_{1}, U_{2} \in \mathcal{U}_{\mathbf{u}}^{\text {in }}$ and $U_{1} \subset \subset U_{2}$ then $\mathbf{u}(\mathbf{x}) \in \operatorname{im}_{\mathrm{T}}\left(\mathbf{u}, U_{1}\right)$ for a.e. $\mathbf{x} \in U_{1}$, and $\mathbf{u}(\mathbf{x}) \notin \operatorname{im}_{\mathrm{T}}\left(\mathbf{u}, U_{1}\right)$ for a.e. $\mathrm{x} \in U_{2} \backslash U_{1}$.

Proof. By [61, Lemma 3.4] (or [43, Lemma 1]), if $\left.\mathbf{u}\right|_{U}$ satisfies INV then $\mathbf{u}$ is one-to-one a.e. in $U$. In order to show the converse it is in fact enough to prove property $b$ ). So let $U_{1}, U_{2} \in \mathcal{U}_{\mathbf{u}}^{\text {in }}$ satisfy $U_{1} \subset \subset U_{2}$. Thus $\chi_{\mathrm{im}_{\mathrm{G}}\left(\mathbf{u}, U_{i}\right)}=\mathcal{N}_{U_{i}}$ a.e. for $i \in\{1,2\}$, and, thanks to equality (5.1),

$$
\chi_{\operatorname{im}_{\mathrm{T}}\left(\mathbf{u}, U_{i}\right)}=\chi_{\mathrm{im}_{\mathrm{G}}\left(\mathbf{u}, U_{i}\right)}=\mathcal{N}_{U_{i}} \quad \text { a.e., } \quad i \in\{1,2\} .
$$

As $\mathbf{u}(\mathbf{x}) \in \operatorname{im}_{\mathrm{G}}\left(\mathbf{u}, U_{1}\right)$ for all $\mathbf{x} \in U_{1} \cap \Omega_{0}$ (recall Definition 2.11), by (5.2) we have that $\mathbf{u}(\mathbf{x}) \in \operatorname{im}_{\mathrm{T}}\left(\mathbf{u}, U_{1}\right.$ ) for a.e. $\mathbf{x} \in U_{1}$. Analogously, by $(5.2), \mathbf{u}(\mathbf{x}) \in \operatorname{im}_{\mathrm{T}}\left(\mathbf{u}, U_{2}\right)$ and $\mathcal{N}_{U_{2}}(\mathbf{u}(\mathbf{x}))=1$ for a.e. $\mathbf{x} \in U_{2}$. As $U_{2} \in \mathcal{U}_{\mathbf{u}}^{\text {in }}$, we must have $\mathcal{N}_{U_{1}}(\mathbf{u}(\mathbf{x}))=0$ for a.e. $\mathbf{x} \in U_{2} \backslash U_{1}$, so by $(5.2), \mathbf{u}(\mathbf{x}) \notin \operatorname{im}_{\mathrm{T}}\left(\mathbf{u}, U_{1}\right)$ for a.e. $\mathbf{x} \in U_{2} \backslash U_{1}$. This concludes the proof.

It follows from [43, Lemma 3] that if $\mathbf{u}: \Omega \rightarrow \mathbb{R}^{n}$ is approximately differentiable a.e. and is one-to-one a.e. in a subset $U \subset \Omega$ then $\left.\mathbf{u}\right|_{U \cap \Omega_{0}}$ is injective, where $\Omega_{0}$ is the set of Definition 2.11. By the definition of geometric image, this means that $\left.\mathbf{u}\right|_{U \cap \Omega_{0}}$ is a bijection from $U \cap \Omega_{0}$ onto $\operatorname{im}_{\mathrm{G}}(\mathbf{u}, U)$. By virtue of (5.1), if $U \in \mathcal{U}_{\mathbf{u}}$, it is then possible to define the local inverse $\left(\left.\mathbf{u}\right|_{U}\right)^{-1}$ in the open set $\operatorname{im}_{\mathrm{T}}(\mathbf{u}, U)$ as follows.

Definition 5.2. Let $\mathbf{u} \in \mathcal{A}_{p}$ and $U \in \mathcal{U}_{\mathbf{u}}^{\text {in }}$. The inverse $\left(\left.\mathbf{u}\right|_{U}\right)^{-1}: \operatorname{im}_{\mathrm{T}}(\mathbf{u}, U) \rightarrow \mathbb{R}^{n}$ is defined a.e. as $\left(\left.\mathbf{u}\right|_{U}\right)^{-1}(\mathbf{y})=\mathbf{x}$, for each $\mathbf{y} \in \operatorname{im}_{G}(\mathbf{u}, U)$, and where $\mathbf{x} \in U \cap \Omega_{0}$ satisfies $\mathbf{u}(\mathbf{x})=\mathbf{y}$.

Since maps in $\mathcal{A}_{p}$ satisfy condition INV locally (by Proposition 4.5 d), Definition 4.4 and Lemma 5.1), we obtain the following result as a particular case of [45, Th. 3.3].

Proposition 5.3. Let $\mathbf{u} \in \mathcal{A}_{p}$ and $U \in \mathcal{U}_{\mathbf{u}}^{\text {in }}$. Then

$$
\left(\left.\mathbf{u}\right|_{U}\right)^{-1} \in W^{1,1}\left(\operatorname{im}_{\mathrm{T}}(\mathbf{u}, U), \mathbb{R}^{n}\right) \quad \text { and } \quad D\left(\left.\mathbf{u}\right|_{U}\right)^{-1}=\left(D \mathbf{u} \circ\left(\left.\mathbf{u}\right|_{U}\right)^{-1}\right)^{-1} \text { a.e. }
$$

\subsection{Monotonicity, continuity and differentiability}

In this subsection we show that maps in $\mathcal{A}_{p}$ satisfy a weak notion of monotonicity and they admit a representative that is continuous $\mathcal{H}^{n-p}$-a.e., differentiable a.e. and satisfies Lusin's condition $N$.

We first identify the boundary of $\operatorname{im}_{\mathrm{T}}(\mathbf{u}, U)$. Recall from Proposition 2.2 the definition of the representative $\mathbf{u}^{*}$ and the set $P$.

Lemma 5.4. Let $p>n-1, \mathbf{u} \in \mathcal{A}_{p}$ and $U \in \mathcal{U}_{\mathbf{u}}$. The following assertions hold. 
a) $\operatorname{im}_{\mathrm{T}}(\mathbf{u}, U) \cup \mathbf{u}(\partial U)$ is closed, $\overline{\operatorname{im}_{\mathrm{T}}(\mathbf{u}, U)} \subset \operatorname{im}_{\mathrm{T}}(\mathbf{u}, U) \cup \mathbf{u}(\partial U)$ and $\partial \operatorname{im}_{\mathrm{T}}(\mathbf{u}, U) \subset \mathbf{u}(\partial U)$.

b) Suppose that $\partial U \cap P=\varnothing$ and $\left.\mathbf{u}^{*}\right|_{\partial U}$ is continuous. Then

$$
\overline{\operatorname{im}_{\mathrm{T}}(\mathbf{u}, U)}=\operatorname{im}_{\mathrm{T}}(\mathbf{u}, U) \cup \mathbf{u}(\partial U) \quad \text { and } \quad \partial \operatorname{im}_{\mathrm{T}}(\mathbf{u}, U)=\mathbf{u}(\partial U) .
$$

Proof. Part a) follows immediately from the continuity of the degree.

Assume the hypotheses of Part b). First note that $U \in \mathcal{U}_{\mathbf{u}^{*}}$, by Remark 2.21. Let $\mathbf{x}_{0} \in \partial U$. By Remark $2.3 \mathrm{~b}), \mathbf{u}^{*}\left(\mathbf{x}_{0}\right)=$ ap $\lim _{\mathbf{x} \rightarrow \mathbf{x}_{0}} \mathbf{u}(\mathbf{x})$, so there exists a sequence $\left\{\mathbf{x}_{j}\right\}_{j \in \mathbb{N}}$ in $U \cap \Omega_{0}$ converging to $\mathbf{x}_{0}$ such that $\underline{\mathbf{u}\left(\mathbf{x}_{j}\right) \rightarrow \mathbf{u}^{*}\left(\mathbf{x}_{0}\right) \text { as } j \rightarrow \infty}$. By Proposition $\left.4.3 e\right), \mathbf{u}\left(\mathbf{x}_{j}\right) \in \overline{\operatorname{im}_{\mathrm{T}}(\mathbf{u}, U)}$ for all $j \in \mathbb{N}$. Consequently, $\mathbf{u}^{*}\left(\mathbf{x}_{0}\right) \in$ $\overline{\operatorname{im}_{\mathrm{T}}(\mathbf{u}, U)}$, which proves $\mathbf{u}^{*}(\partial U) \subset \overline{\operatorname{im}_{\mathrm{T}}(\mathbf{u}, U)}$. But by Convention 2.14 and Remark 2.19, $\mathbf{u}^{*}(\partial U)=\mathbf{u}(\partial U)$. Finally, as $\operatorname{im}_{\mathrm{T}}(\mathbf{u}, U)$ is open and does not intersect $\mathbf{u}(\partial U)$, we also have $\partial \operatorname{im}_{\mathrm{T}}(\mathbf{u}, U)=\mathbf{u}(\partial U)$.

Now we show that maps in $\mathcal{A}_{p}$ satisfy a version of the maximum and minimum principles. For each $i \in\{1, \ldots, n\}$, we denote by $u^{i}$ the $i$ th component of $\mathbf{u}$.

Proposition 5.5. Let $\mathbf{u} \in \mathcal{A}_{p}, U \in \mathcal{U}_{\mathbf{u}}$ and $i \in\{1, \ldots, n\}$. Then,

$$
\min u^{i}(\partial U) \leq u^{i}(\mathbf{x}) \leq \max u^{i}(\partial U) \text { a.e. } \mathbf{x} \in U .
$$

Proof. Thanks to Lemma $2.8 c$ ) and equality (5.1), $\mathbf{u}(\mathbf{x}) \in \operatorname{im}_{\mathrm{T}}(\mathbf{u}, U)$ for a.e. $\mathbf{x} \in U$. Denote by $\pi^{i}: \mathbb{R}^{n} \rightarrow \mathbb{R}$ the projection onto the $i$ th coordinate. Then, using also that projections are open maps, as well as Lemma $5.4 a$ ), we obtain

$$
\underset{U}{\operatorname{ess} \sup } u^{i} \leq \sup \pi^{i}\left(\operatorname{im}_{\mathrm{T}}(\mathbf{u}, U)\right)=\sup \partial\left(\pi^{i}\left(\operatorname{im}_{\mathrm{T}}(\mathbf{u}, U)\right)\right) \leq \sup \pi^{i}\left(\partial \operatorname{im}_{\mathrm{T}}(\mathbf{u}, U)\right) \leq \sup \pi^{i}(\mathbf{u}(\partial U)),
$$

where ess sup denotes the essential supremum. This shows that $u^{i}(\mathbf{x}) \leq \max u^{i}(\partial U)$ for a.e. $\mathbf{x} \in U$, and the other inequality of (5.3) is proved analogously.

Property (5.3) is closely related to Manfredi's [56] notion of weak monotonicity and earlier related definitions (see, e.g., [73]). In fact, one can see, e.g., from the proof of [47, Th. 2.17] that a Sobolev map satisfying $\operatorname{det} D \mathbf{u}>0$ a.e. and $\operatorname{Det} D \mathbf{u}=\operatorname{det} D \mathbf{u}$ is weakly monotone. Also, our property (5.3) means that $\mathbf{u}$ is weakly pseudomonotone in the sense of [41, Sect. 2].

It is well-known (see, e.g., [47, Ch. 2]) that the above notions of monotonicity imply regularity properties for the map: in particular, a representative of $\mathbf{u}$ is continuous $\mathcal{H}^{n-p}$-a.e. (if $p \leq n$ ) and differentiable a.e. However, we will not deal with the representative normally used in the theory of monotone maps (see, e.g., $[70,56,72,41,47])$ but rather with the one defined in [61, Th. 7.4], which we explain in the following paragraphs.

Definition 5.6. Let $\mathbf{u} \in \mathcal{A}_{p}$. We define the topological image of a point $\mathbf{x} \in \Omega$ by $\mathbf{u}$ as

$$
\operatorname{im}_{\mathrm{T}}(\mathbf{u}, \mathbf{x}):=\bigcap_{\substack{r>0 \\ B(\mathbf{x}, r) \in \mathcal{U}_{\mathbf{u}}}} \overline{\operatorname{im}_{\mathrm{T}}(\mathbf{u}, B(\mathbf{x}, r))},
$$

and $N C:=\left\{\mathbf{x} \in \Omega: \mathcal{H}^{0}\left(\operatorname{im}_{\mathrm{T}}(\mathbf{u}, \mathbf{x})\right)>1\right\}$.

Remark 5.7. a) The topological image of a point is compact and non-empty, since it is defined as a decreasing intersection of compact and non-empty sets. The fact that the intersection is decreasing is a consequence of Proposition $4.3 \mathrm{~d}$ ), and the fact that the sets involved are non-empty is a consequence of, for example, Proposition $4.3 \mathrm{e}$ ). 
b) We have $\mathcal{L}^{n}\left(\operatorname{im}_{\mathrm{T}}(\mathbf{u}, \mathbf{x})\right)=0$. Indeed, this is an immediate consequence of [44, Th. 4.6], but we provide a brief self-contained proof. By Lemma $5.4 a$ ), equality (5.1) and Remark $2.16 \mathrm{~b}$ ), we obtain that

$$
\begin{aligned}
\mathcal{L}^{n}\left(\operatorname{im}_{\mathrm{T}}(\mathbf{u}, \mathbf{x})\right) & =\inf _{\substack{r>0 \\
B(\mathbf{x}, r) \in \mathcal{U}_{\mathbf{u}}}} \mathcal{L}^{n}\left(\overline{\operatorname{im}_{\mathrm{T}}(\mathbf{u}, B(\mathbf{x}, r))}\right) \leq \inf _{\substack{r>0 \\
(\mathbf{x}, r) \in \mathcal{U}_{\mathbf{u}}}} \mathcal{L}^{n}\left(\operatorname{im}_{\mathrm{G}}(\mathbf{u}, B(\mathbf{x}, r))\right. \\
& =\mathcal{L}^{n}\left(\bigcap_{\substack{r>0 \\
B(\mathbf{x}, r) \in \mathcal{U}_{\mathbf{u}}}} \operatorname{im}_{\mathrm{G}}(\mathbf{u}, B(\mathbf{x}, r))\right)=\mathcal{L}^{n}\left(\operatorname{im}_{\mathrm{G}}(\mathbf{u},\{\mathbf{x}\})\right)=0 .
\end{aligned}
$$

c) Given $\mathbf{u}_{1}, \mathbf{u}_{2} \in \mathcal{A}_{p}$ that coincide a.e. and $U \in \mathcal{U}_{\mathbf{u}_{1}}$, by Lemma 2.20 , for each $\mathbf{x} \in \Omega$ there is $r>0$ with $B(\mathbf{x}, r) \in \mathcal{U}_{\mathbf{u}_{1}} \cap \mathcal{U}_{\mathbf{u}_{2}}$ such that $B(\mathbf{x}, r) \subset \subset U$ and $\mathbf{u}_{1}=\mathbf{u}_{2} \mathcal{H}^{n-1}$-a.e. in $\partial B(\mathbf{x}, r)$. Then, by Proposition $4.3 d)$,

$$
\overline{\operatorname{im}_{\mathrm{T}}\left(\mathbf{u}_{2}, B(\mathbf{x}, r)\right)}=\overline{\operatorname{im}_{\mathrm{T}}\left(\mathbf{u}_{1}, B(\mathbf{x}, r)\right)} \subset \overline{\operatorname{im}_{\mathrm{T}}\left(\mathbf{u}_{1}, U\right)} .
$$

Consequently, neither the topological image of a point nor the set $N C$ depend on the particular representative of $\mathbf{u}$.

d) As observed in a), the definition of $\operatorname{im}_{\mathrm{T}}(\mathbf{u}, \mathbf{x})$ involves a decreasing intersection. Moreover, the theory of monotone maps ensures that there exists a representative $\overline{\mathbf{u}}$ of $\mathbf{u}$ that is continuous $\mathcal{H}^{\max \{n-p, 0\}}$-a.e., and, as mentioned in $\mathrm{c}), \mathrm{im}_{\mathrm{T}}(\mathbf{u}, \mathbf{x})$ does not depend on the representative. Consequently, $\operatorname{im}_{\mathrm{T}}(\mathbf{u}, \mathbf{x})$ also equals the intersection of $\operatorname{im}_{\mathrm{T}}(\overline{\mathbf{u}}, B(\mathbf{x}, r))$ when $r>0$ satisfies $B(\mathbf{x}, r) \in \mathcal{U}_{\overline{\mathbf{u}}} \cap \mathcal{U}_{\mathbf{u}}$ and $\overline{\mathbf{u}}$ is continuous in $\partial B(\mathbf{x}, r)$. But for those $r$ we have, thanks to Lemma $5.4 b)$ and Convention 2.14,

$$
\overline{\operatorname{im}_{\mathrm{T}}(\overline{\mathbf{u}}, U)}=\operatorname{im}_{\mathrm{T}}(\overline{\mathbf{u}}, U) \cup \overline{\mathbf{u}}(\partial U)=\operatorname{im}_{\mathrm{T}}(\mathbf{u}, U) \cup \mathbf{u}(\partial U) .
$$

Therefore, Definition 5.6 coincides with Šverák's [70, pp. 114-115] (see also [61, Eqs. (7.4) and (7.13)]).

Following [61, Sect. 7], we define a representative that enjoys better properties than that of Proposition 2.2 .

Definition 5.8. Let $p>n-1$. Given $\mathbf{u} \in \mathcal{A}_{p}$, we define

$$
\hat{\mathbf{u}}(\mathbf{x}):= \begin{cases}\mathbf{u}^{*}(\mathbf{x}) & \text { if } \mathbf{x} \in \Omega \backslash(P \cup N C), \\ \text { any element of } \operatorname{im}_{\mathrm{T}}(\mathbf{u}, \mathbf{x}) & \text { if } \mathbf{x} \in P \cup N C .\end{cases}
$$

Note that $\hat{\mathbf{u}}$ is defined everywhere in $\Omega$. The definition in $P \cup N C$ is possible due to the axiom of choice.

Proposition 5.9. Let $p>n-1$ and $\mathbf{u} \in \mathcal{A}_{p}$. Then $\hat{\mathbf{u}}(\mathbf{x}) \in \operatorname{im}_{\mathrm{T}}(\mathbf{u}, \mathbf{x})$ for every $\mathbf{x} \in \Omega, \mathcal{H}^{\max \{n-p, 0\}}(N C)=0$, $\hat{\mathbf{u}}$ is continuous at every point of $\Omega \backslash N C$, and $\hat{\mathbf{u}}$ is differentiable a.e. Moreover, if $N \subset \Omega$ satisfies $\mathcal{L}^{n}(N)=0$ then $\mathcal{L}^{n}(\hat{\mathbf{u}}(N))=0$.

Proof. The facts $\hat{\mathbf{u}}(\mathbf{x}) \in \operatorname{im}_{\mathrm{T}}(\mathbf{u}, \mathbf{x})$ for every $\mathbf{x} \in \Omega, \mathcal{H}^{\max \{n-p, 0\}}(N C)=0$ and $\hat{\mathbf{u}}$ is continuous at every point of $\Omega \backslash N C$ can be established exactly as in Müller \& Spector [61, Th. 7.4]. They assume condition INV for $\mathbf{u}$, but all that matters is [61, Lemma 7.3(i)], which is our Proposition $4.3 d$ ). Recall also from Remark 5.7.d) that our definition of $\hat{\mathbf{u}}$ coincides with theirs. Notice, in addition, that they assume $p<n$. If $p>n$, Morrey's embedding theorem implies that $\mathbf{u}^{*}$ is continuous and, consequently, $P=\varnothing$, whereas the proof of [61, Th. 7.4] also shows that $\mathbf{u}^{*}$ is discontinuous at $N C$, so $N C=\varnothing$ in this case. If $p=n$, as $\operatorname{det} D \mathbf{u}>0$ a.e., thanks to [73] (see also all the theory of monotone maps cited at the beginning of the Section), $\mathbf{u}^{*}$ is continuous, so again $P \cup N C=\varnothing$.

We deal now with the differentiability a.e. As before, for $p>n$, we have that $\hat{\mathbf{u}}=\mathbf{u}^{*}$, and it is a well-known result in Sobolev spaces (see, e.g., [29, Sect. 6.2]) that $\mathbf{u}^{*}$ is differentiable a.e. For $p=n$, again due to the assumption $\operatorname{det} D \mathbf{u}>0$ a.e., we have $\hat{\mathbf{u}}=\mathbf{u}^{*}$ and the differentiability a.e. of $\mathbf{u}^{*}$ is covered by the theory of monotone functions (see, e.g., [68, Sect. III.4.3] or [47, Th. 2.24]).

Therefore, we can assume that $p<n$. The first step consists of the following oscillation estimate: mimicking the proof of [61, Th. 7.4] (or else those of the theory of weakly monotone functions; see, e.g., [56, 
Th. 1], [41, Th. 4.1] or [47, Th. 2.24]) we obtain that there exists $C>0$ such that for all $i \in\{1, \ldots, n\}$, $\mathbf{x}_{0} \in \Omega$ and $r>0$ for which $B\left(\mathbf{x}_{0}, 2 r\right) \subset \Omega$, we have

$$
\underset{B\left(\mathbf{x}_{0}, r\right)}{\operatorname{ess} \sup } u^{i}-\underset{B\left(\mathbf{x}_{0}, r\right)}{\operatorname{ess} \inf } u^{i} \leq C r\left(f_{B\left(\mathbf{x}_{0}, 2 r\right)}\left|D u^{i}\right|^{p} \mathrm{~d} \mathbf{x}\right)^{\frac{1}{p}} .
$$

Said otherwise, for a.e. $\mathbf{x}_{1}, \mathbf{x}_{2} \in B\left(\mathbf{x}_{0}, r\right)$,

$$
\left|\mathbf{u}\left(\mathbf{x}_{2}\right)-\mathbf{u}\left(\mathbf{x}_{1}\right)\right| \leq C r\left(f_{B\left(\mathbf{x}_{0}, 2 r\right)}|D \mathbf{u}|^{p} \mathrm{~d} \mathbf{x}\right)^{\frac{1}{p}} .
$$

Let $\mathbf{x}_{1} \in \Omega \backslash(P \cup N C)$ be a Lebesgue point of $|\nabla \mathbf{u}|^{p}$, and let $\mathbf{x}_{2} \in \Omega \backslash(P \cup N C)$ satisfy $B\left(\mathbf{x}_{1}, 2\left|\mathbf{x}_{2}-\mathbf{x}_{1}\right|\right) \subset \Omega$. Then, recalling (2.1) and using (5.4),

$$
\begin{aligned}
\left|\hat{\mathbf{u}}\left(\mathbf{x}_{2}\right)-\hat{\mathbf{u}}\left(\mathbf{x}_{1}\right)\right| & =\left|\lim _{r \searrow 0}\left(f_{B\left(\mathbf{x}_{2}, r\right)} \mathbf{u} \mathrm{d} \mathbf{x}-f_{B\left(\mathbf{x}_{1}, r\right)} \mathbf{u} \mathrm{d} \mathbf{x}\right)\right| \leq \liminf _{r \searrow 0} f_{B(\mathbf{0}, 1)}\left|\mathbf{u}\left(\mathbf{x}_{2}+r \mathbf{z}\right)-\mathbf{u}\left(\mathbf{x}_{1}+r \mathbf{z}\right)\right| \mathrm{d} \mathbf{z} \\
& \leq C\left|\mathbf{x}_{2}-\mathbf{x}_{1}\right|\left(f_{B\left(\mathbf{x}_{1}, 2\left|\mathbf{x}_{2}-\mathbf{x}_{1}\right|\right)}|D \mathbf{u}|^{p} \mathrm{~d} \mathbf{x}\right)^{\frac{1}{p}},
\end{aligned}
$$

so

$$
\limsup _{\substack{\mathbf{x}_{2} \rightarrow \mathbf{x}_{1} \\ \mathbf{x}_{2} \notin P \cup N C}} \frac{\left|\hat{\mathbf{u}}\left(\mathbf{x}_{2}\right)-\hat{\mathbf{u}}\left(\mathbf{x}_{1}\right)\right|}{\left|\mathbf{x}_{2}-\mathbf{x}_{1}\right|}<\infty .
$$

Now let $\mathbf{x}_{2} \in P \cup N C$. By Definition 5.6 and equality (5.1), there exists $\mathbf{x}_{3} \in \Omega_{\hat{\mathbf{u}}, 0} \cap B\left(\mathbf{x}_{2},\left|\mathbf{x}_{2}-\mathbf{x}_{1}\right|\right) \backslash(P \cup N C)$ such that $\left|\hat{\mathbf{u}}\left(\mathbf{x}_{2}\right)-\hat{\mathbf{u}}\left(\mathbf{x}_{3}\right)\right| \leq\left|\mathbf{x}_{2}-\mathbf{x}_{1}\right|$. Thus,

$$
\frac{\left|\hat{\mathbf{u}}\left(\mathbf{x}_{2}\right)-\hat{\mathbf{u}}\left(\mathbf{x}_{1}\right)\right|}{\left|\mathbf{x}_{2}-\mathbf{x}_{1}\right|} \leq \frac{\left|\hat{\mathbf{u}}\left(\mathbf{x}_{2}\right)-\hat{\mathbf{u}}\left(\mathbf{x}_{3}\right)\right|}{\left|\mathbf{x}_{2}-\mathbf{x}_{1}\right|}+\frac{\left|\hat{\mathbf{u}}\left(\mathbf{x}_{3}\right)-\hat{\mathbf{u}}\left(\mathbf{x}_{1}\right)\right|}{\left|\mathbf{x}_{3}-\mathbf{x}_{1}\right|} \frac{\left|\mathbf{x}_{3}-\mathbf{x}_{1}\right|}{\left|\mathbf{x}_{2}-\mathbf{x}_{1}\right|} \leq 1+2 \frac{\left|\hat{\mathbf{u}}\left(\mathbf{x}_{3}\right)-\hat{\mathbf{u}}\left(\mathbf{x}_{1}\right)\right|}{\left|\mathbf{x}_{3}-\mathbf{x}_{1}\right|}
$$

so (5.5) shows that

$$
\limsup _{\substack{\mathbf{x}_{2} \rightarrow \mathbf{x}_{1} \\ \mathbf{x}_{2} \in P \cup N C}} \frac{\left|\hat{\mathbf{u}}\left(\mathbf{x}_{2}\right)-\hat{\mathbf{u}}\left(\mathbf{x}_{1}\right)\right|}{\left|\mathbf{x}_{2}-\mathbf{x}_{1}\right|} \leq 1+2 \limsup _{\substack{\mathbf{x}_{3} \rightarrow \mathbf{x}_{1} \\ \mathbf{x}_{3} \notin P \cup N C}} \frac{\left|\hat{\mathbf{u}}\left(\mathbf{x}_{3}\right)-\hat{\mathbf{u}}\left(\mathbf{x}_{1}\right)\right|}{\left|\mathbf{x}_{3}-\mathbf{x}_{1}\right|}<\infty .
$$

Altogether, (5.5) and (5.6) yield that

$$
\limsup _{\mathbf{x}_{2} \rightarrow \mathbf{x}_{1}} \frac{\left|\hat{\mathbf{u}}\left(\mathbf{x}_{2}\right)-\hat{\mathbf{u}}\left(\mathbf{x}_{1}\right)\right|}{\left|\mathbf{x}_{2}-\mathbf{x}_{1}\right|}<\infty
$$

for all $\mathbf{x}_{1} \in \Omega \backslash(P \cup N C)$ that is Lebesgue point of $|\nabla \mathbf{u}|^{p}$. Stepanov's theorem (see, e.g., [47, Th. 2.23]) concludes the differentiability a.e.

Finally, the fact that $\mathcal{L}^{n}(\hat{\mathbf{u}}(N))=0$ for any $N \subset \Omega$ with $\mathcal{L}^{n}(N)=0$ can be proved exactly as in [61, Th. 10.1] (in fact, slightly shorter because here we have the additional assumption $\operatorname{Det} D \mathbf{u}=\operatorname{det} D \mathbf{u}$ ). This finishes the proof.

Remark 5.10. As an immediate consequence of Proposition 5.9, we have that $P \subset N C$ and

$$
\hat{\mathbf{u}}(\mathbf{x})= \begin{cases}\mathbf{u}^{*}(\mathbf{x}) & \text { if } \mathbf{x} \in \Omega \backslash N C, \\ \text { any element of } \operatorname{im}_{\mathrm{T}}(\mathbf{u}, \mathbf{x}) & \text { if } \mathbf{x} \in N C .\end{cases}
$$

Note also that if $U \in \mathcal{U}_{\mathbf{u}}$ and $\partial U \cap N C=\varnothing$ then $\partial U \cap P=\varnothing$ and $\left.\mathbf{u}^{*}\right|_{\partial U}$ is continuous, so the assumptions of Lemma $5.4 b$ ) are satisfied. 
We will see that the restriction $\partial U \cap N C=\varnothing$ for a $U \in \mathcal{U}_{\mathbf{u}}$ plays an important role in the sequel. Accordingly, we set the following definition.

Definition 5.11. Let $\mathbf{u} \in \mathcal{A}_{p}$. Define $\mathcal{U}_{\mathbf{u}}^{N}:=\left\{U \in \mathcal{U}_{\mathbf{u}}: \partial U \cap N C=\varnothing\right\}$ and $\mathcal{U}_{\mathbf{u}}^{N \text {,in }}:=\mathcal{U}_{\mathbf{u}}^{N} \cap \mathcal{U}_{\mathbf{u}}^{\text {in }}$.

As a consequence of Lemma 2.20 and Proposition 5.9, the following result holds.

Lemma 5.12. Let $\mathbf{u} \in \mathcal{A}_{p}$. Let $U \subset \subset \Omega$ be a non-empty open set with a $C^{2}$ boundary. Then there exists $\delta>0$ such that $U_{t} \in \mathcal{U}_{\mathbf{u}}^{N}$ for a.e. $t \in(-\delta, \delta)$, where $U_{t}$ is defined as in (2.2). Moreover, for each compact $K \subset \Omega$ there exists $U^{\prime} \in \mathcal{U}_{\mathbf{u}}^{N}$ such that $K \subset U^{\prime}$.

\section{$5.3 \quad \mathcal{H}^{n-1}$-continuity of the local inverse}

In this subsection we define a precise representative of the local inverse of Definition 5.2. Given $U \in \mathcal{U}_{\mathbf{u}}^{N \text {,in }}$, we shall show that $\mathcal{H}^{n-1}$-a.e. $\mathbf{y} \in \operatorname{im}_{\mathrm{T}}(\mathbf{u}, U)$ has only one preimage in $U$ by $\hat{\mathbf{u}}$, so we can define a precise representative of the inverse $\left(\left.\mathbf{u}\right|_{U}\right)^{-1}$, which is fact is continuous at any such $\mathbf{y}$. This subsection is not used elsewhere in the paper and may be omitted in a first reading.

In order to prove these results, we follow Šverák [70] and set, for every $\mathbf{u} \in \mathcal{A}_{p}, U \subset \subset \Omega, \mathbf{y} \in \mathbb{R}^{n}$ and $K \subset \mathbb{R}^{n}$,

$$
G_{U}(\mathbf{y}):=\left\{\mathbf{x} \in \bar{U}: \mathbf{y} \in \operatorname{im}_{\mathrm{T}}(\mathbf{u}, \mathbf{x})\right\}, \quad G_{U}(K):=\bigcup_{\mathbf{y} \in K} G_{U}(\mathbf{y}) .
$$

First we show some general results of the set-valued function $G_{U}$ that do not require invertibility.

Lemma 5.13. Let $\mathbf{u} \in \mathcal{A}_{p}$ and $U \in \mathcal{U}_{\mathbf{u}}^{N}$. The following properties hold:

a) If $\mathbf{y} \in \operatorname{im}_{\mathrm{T}}(\mathbf{u}, U)$, then $G_{U}(\mathbf{y}) \subset U$.

b) If $A \subset \mathbb{R}^{n}$ is open, then $A^{*}:=\left\{\mathbf{x} \in \Omega: \operatorname{im}_{\mathrm{T}}(\mathbf{u}, \mathbf{x}) \subset A\right\}$ is open.

c) If $K \subset \Omega$ is compact, then $\tilde{K}:=\bigcup_{\mathbf{x} \in K} \operatorname{im}_{\mathrm{T}}(\mathbf{u}, \mathbf{x})$ is compact.

d) If $K \subset \mathrm{im}_{\mathrm{T}}(\mathbf{u}, U)$ is compact, then $G_{U}(K)$ is compact.

e) Consider the set

$$
Y:=\bigcup_{\mathbf{x} \in U} \operatorname{im}_{\mathrm{T}}(\mathbf{u}, \mathbf{x})
$$

Then $\operatorname{im}_{\mathrm{T}}(\mathbf{u}, U) \subset Y$ and $G_{U}(\mathbf{y}) \cap U \neq \varnothing$ for every $\mathbf{y} \in \operatorname{im}_{\mathrm{T}}(\mathbf{u}, U)$.

Proof. Part a). It suffices to note that $\mathbf{y} \notin \mathbf{u}(\partial U)$ and that if $\mathbf{x} \in \partial U$, then $\operatorname{im}_{\mathrm{T}}(\mathbf{u}, \mathbf{x})=\{\mathbf{u}(\mathbf{x})\}$, since $\partial U \cap N C=\varnothing$.

Part b). Let $\mathbf{x}_{0} \in A^{*}$. As $\operatorname{im}_{\mathrm{T}}\left(\mathbf{u}, \mathbf{x}_{0}\right) \subset A$ and $\operatorname{im}_{\mathrm{T}}\left(\mathbf{u}, \mathbf{x}_{0}\right)$ was defined as a decreasing intersection of compact sets, there exists $r_{0}>0$ such that $B\left(\mathbf{x}_{0}, r_{0}\right) \in \mathcal{U}_{\mathbf{u}}$ and

$$
\operatorname{im}_{\mathrm{T}}\left(\mathbf{u}, \mathbf{x}_{0}\right) \subset \overline{\operatorname{im}_{\mathrm{T}}\left(\mathbf{u}, B\left(\mathbf{x}_{0}, r_{0}\right)\right)} \subset A .
$$

Take $\mathbf{x} \in B\left(\mathbf{x}_{0}, \frac{r_{0}}{2}\right) \backslash\left\{\mathbf{x}_{0}\right\}$. Then $B\left(\mathbf{x},\left|\mathbf{x}-\mathbf{x}_{0}\right|\right) \subset B\left(\mathbf{x}_{0}, r_{0}\right)$ so, by Proposition $\left.4.3 d\right)$,

$$
\overline{\operatorname{im}_{\mathrm{T}}(\mathbf{u}, B(\mathbf{x}, r))} \subset \overline{\operatorname{im}_{\mathrm{T}}\left(\mathbf{u}, B\left(\mathbf{x}_{0}, r_{0}\right)\right)},
$$

for any $r \in\left(0,\left|\mathbf{x}-\mathbf{x}_{0}\right|\right)$ such that $B(\mathbf{x}, r) \in \mathcal{U}_{\mathbf{u}}$, and, hence,

$$
\operatorname{im}_{\mathrm{T}}(\mathbf{u}, \mathbf{x})=\bigcap_{\substack{0<r<\left|\mathbf{x}-\mathbf{x}_{0}\right| \\ B(\mathbf{x}, r) \in \mathcal{U}_{\mathbf{u}}}} \overline{\operatorname{im}_{\mathrm{T}}(\mathbf{u}, B(\mathbf{x}, r))} \subset \overline{\operatorname{im}_{\mathrm{T}}\left(\mathbf{u}, B\left(\mathbf{x}_{0}, r_{0}\right)\right)} \subset A .
$$

Consequently, $\mathbf{x} \in A^{*}$ and $B\left(\mathbf{x}_{0}, \frac{r_{0}}{2}\right) \subset A^{*}$. 
Part c). Consider an open covering $\mathcal{A}$ of $\tilde{K}$; as $\tilde{K} \subset \operatorname{im}_{\mathrm{T}}\left(\mathbf{u}, U^{\prime}\right)$. Since for each $\mathbf{x} \in K$ the $\operatorname{set}_{\mathrm{im}}(\mathbf{u}, \mathbf{x})$ is compact, there exists a finite family $\mathcal{A}_{\mathbf{x}} \subset \mathcal{A}$ that covers $\operatorname{im}_{\mathrm{T}}(\mathbf{u}, \mathbf{x})$. Call $A_{\mathbf{x}}:=\bigcup \mathcal{A}_{\mathbf{x}}$. Then $\operatorname{im}_{\mathrm{T}}(\mathbf{u}, \mathbf{x}) \subset A_{\mathbf{x}}$, the family $\mathcal{A}^{\prime}:=\left\{A_{\mathbf{x}}: \mathbf{x} \in K\right\}$ is also an open covering of $\tilde{K}$, and $\mathbf{x} \in A_{\mathbf{x}}^{*}$, thanks to part $b$ ). Therefore, the family $\mathcal{A}^{*}:=\left\{A_{\mathbf{x}}^{*}: \mathbf{x} \in K\right\}$ is an open covering of $K$. Thus, there exist $p \in \mathbb{N}$ and $\mathbf{x}_{1}, \ldots, \mathbf{x}_{p} \in K$ such that

$$
K \subset \bigcup_{i=1}^{p} A_{\mathbf{x}_{i}}^{*}
$$

Now, for any $\mathbf{y} \in \tilde{K}$ there exists $\mathbf{x} \in K$ such that $\mathbf{y} \in \operatorname{im}_{\mathrm{T}}(\mathbf{u}, \mathbf{x})$, so $\mathbf{x} \in A_{\mathbf{x}_{i}}^{*}$ for some $i \in\{1, \ldots, p\}$, and, hence, $\operatorname{im}_{\mathrm{T}}(\mathbf{u}, \mathbf{x}) \subset A_{\mathbf{x}_{i}}$. This shows that

$$
\tilde{K} \subset \bigcup_{i=1}^{p} A_{\mathbf{x}_{i}}=\bigcup_{i=1}^{p} \bigcup \mathcal{A}_{\mathbf{x}_{i}}
$$

which proves the compactness of $\tilde{K}$.

Part d). Suppose that $\left\{\mathbf{x}_{k}\right\}_{k \in \mathbb{N}} \subset \bar{U}$ and $\left\{\mathbf{y}_{k}\right\}_{k \in \mathbb{N}} \subset K$ are such that $\mathbf{y}_{k} \in \operatorname{im}_{\mathrm{T}}\left(\mathbf{u}, \mathbf{x}_{k}\right)$ for all $k \in \mathbb{N}$. Passing to a subsequence, we can assume that $\mathbf{x}_{k} \rightarrow \mathbf{x}_{0}$ and $\mathbf{y}_{k} \rightarrow \mathbf{y}_{0}$ as $k \rightarrow \infty$, for some $\mathbf{x}_{0} \in \bar{U}$ and $\mathbf{y}_{0} \in K$. We shall prove that $\mathbf{x}_{0} \in G_{U}(K)$. By Proposition $\left.4.3 d\right)$, for any $r>0$ such that $B\left(\mathbf{x}_{0}, r\right) \in \mathcal{U}_{\mathbf{u}}$ there exists $k_{r} \in \mathbb{N}$ for which for all $k \geq k_{r}$,

$$
\mathbf{y}_{k} \in \operatorname{im}_{\mathrm{T}}\left(\mathbf{u}, \mathbf{x}_{k}\right) \subset \overline{\operatorname{im}_{\mathrm{T}}\left(\mathbf{u}, B\left(\mathbf{x}_{0}, r\right)\right)} .
$$

Hence $\mathbf{y}_{0} \in \overline{\operatorname{im}_{\mathrm{T}}\left(\mathbf{u}, B\left(\mathbf{x}_{0}, r\right)\right)}$, and, consequently, $\mathbf{y}_{0} \in \operatorname{im}_{\mathrm{T}}\left(\mathbf{u}, \mathbf{x}_{0}\right)$. It follows that $\mathbf{x}_{0} \in G_{U}\left(\mathbf{y}_{0}\right) \subset G_{U}(K)$.

Part e). Let $Y^{\prime}:=\bigcup_{\mathbf{x} \in \bar{U}} \operatorname{im}_{\mathrm{T}}(\mathbf{u}, \mathbf{x})$. Since $\operatorname{im}_{\mathrm{T}}(\mathbf{u}, \mathbf{x})=\{\mathbf{u}(\mathbf{x})\}$ for every $\mathbf{x} \in \partial U$, we can write $Y^{\prime}=$ $Y \cup \mathbf{u}(\partial U)$. Therefore, it is enough to show the inclusion $\operatorname{im}_{\mathrm{T}}(\mathbf{u}, U) \subset Y^{\prime}$. Since $Y^{\prime}$ is compact by part $\left.c\right)$, the set $H:=\operatorname{im}_{\mathrm{T}}(\mathbf{u}, U) \backslash Y^{\prime}$ is open.

On the other hand, for all $\mathbf{x} \in U$, as $\operatorname{im}_{\mathrm{T}}(\mathbf{u}, \mathbf{x}) \cap H=\varnothing$, by Remark 5.10 we have $\hat{\mathbf{u}}(\mathbf{x}) \notin H$. In other words, $\hat{\mathbf{u}}(U) \cap H=\varnothing$. By Lemma 2.8 a) and Proposition 5.9 we have $\mathcal{L}^{n}\left(\operatorname{im}_{G}(\mathbf{u}, U) \cap H\right)=0$, and by (5.1), $\mathcal{L}^{n}\left(\operatorname{im}_{\mathrm{T}}(\mathbf{u}, U) \cap H\right)=0$, so $\mathcal{L}^{n}(H)=0$. As $H$ is open, we must have $H=\varnothing$. Therefore $\operatorname{im}_{\mathrm{T}}(\mathbf{u}, U) \subset Y$ and, as a consequence, $G_{U}(\mathbf{y}) \cap U \neq \varnothing$ for every $\mathbf{y} \in \operatorname{im}_{\mathrm{T}}(\mathbf{u}, U)$.

The following result will allow us to define a precise representative of the inverse. Given $\mathbf{u} \in \mathcal{A}_{p}$ and $U \in \mathcal{U}_{\mathbf{u}}^{N}$, we denote by $U_{+}$the set of the points in $U$ where $\hat{\mathbf{u}}$ has a regular approximate differential with positive Jacobian.

Proposition 5.14. Let $\mathbf{u} \in \mathcal{A}_{p}$ and $U \in \mathcal{U}_{\mathbf{u}}^{N \text {,in }}$. The following properties hold.

a) If $\mathbf{y} \in \operatorname{im}_{\mathrm{T}}(\mathbf{u}, U)$, then $G_{U}(\mathbf{y})$ is connected.

b) If $\mathbf{x} \in U_{+}$and $\hat{\mathbf{u}}(\mathbf{x}) \in \operatorname{im}_{\mathrm{T}}(\mathbf{u}, U)$, then $G_{U}(\hat{\mathbf{u}}(\mathbf{x}))=\{\mathbf{x}\}$.

c) The set

$$
T:=\left\{\mathbf{y} \in \operatorname{im}_{\mathrm{T}}(\mathbf{u}, U): \mathcal{H}^{0}\left(G_{U}(\mathbf{y})\right)>1\right\}
$$

has null $\mathcal{H}^{n-1}$-measure, while $\mathcal{L}^{n}\left(G_{U}(T)\right)=0$.

d) The set

$$
T^{\prime}:=\bigcup_{\mathbf{x} \in U \cap N C} \operatorname{im}_{\mathrm{T}}(\mathbf{u}, \mathbf{x}) \cap \operatorname{im}_{\mathrm{T}}(\mathbf{u}, U)
$$

has null measure. 
Proof. For simplicity of notation, we assume, without loss of generality, that $\mathbf{u}=\hat{\mathbf{u}}$.

Part a). Assume, for a contradiction, that $G_{U}(\mathbf{y})$ is not connected. Since, by Lemma $5.13, G_{U}(\mathbf{y})$ is compact and contained in $U$, we can write it as $G_{U}(\mathbf{y})=K_{1} \cup K_{2}$, where $K_{1}$ and $K_{2}$ are two non-empty, compact and disjoint subsets of $U$. By Lemma 5.12, we can find two disjoint sets $U_{1}, U_{2} \in \mathcal{U}_{\mathbf{u}}^{N}$ such that $K_{i} \subset U_{i} \subset U$ for $i \in\{1,2\}$.

Assume that $\mathbf{y}=\mathbf{u}(\mathbf{x})$ for some $\mathbf{x} \in \partial U_{i}$ and some $i \in\{1,2\}$. Since $\operatorname{im}_{\mathrm{T}}(\mathbf{u}, \mathbf{x})=\{\mathbf{u}(\mathbf{x})\}$ we have that $\mathbf{x} \in G_{U}(\mathbf{y})$, so $\mathbf{x} \in K_{i}$ with $K_{i} \cap \partial U_{i}=\varnothing$, a contradiction. Therefore, $\mathbf{y} \notin \mathbf{u}\left(\partial U_{1} \cup \partial U_{2}\right)$. Now take $\mathbf{x}_{i} \in K_{i}$ for each $i \in\{1,2\}$. Then $\mathbf{y} \in \operatorname{im}_{\mathrm{T}}\left(\mathbf{u}, \mathbf{x}_{i}\right)$, so by Definition 5.6 and Proposition $\left.4.3 d\right), \mathbf{y} \in \overline{\operatorname{im}_{\mathrm{T}}\left(\mathbf{u}, U_{i}\right)}$, but by Lemma 5.4, $\mathbf{y} \in \operatorname{im}_{\mathrm{T}}\left(\mathbf{u}, U_{i}\right)$. Therefore, $\mathbf{y} \in \operatorname{im}_{\mathrm{T}}\left(\mathbf{u}, U_{1}\right) \cap \mathrm{im}_{\mathrm{T}}\left(\mathbf{u}, U_{2}\right)$. Now we argue as in the proof of Proposition 4.5 a). By Proposition $4.3 \mathrm{c}), \operatorname{deg}\left(\mathbf{u}, U_{i}, \mathbf{y}\right) \geq 1$ for each $i \in\{1,2\}$, so, by the additivity of the degree and Proposition $4.3 d$ ),

$$
2 \leq \operatorname{deg}\left(\mathbf{u}, U_{1} \cup U_{2}, \mathbf{y}\right) \leq \operatorname{deg}(\mathbf{u}, U, \mathbf{y}) .
$$

Therefore, $\operatorname{deg}(\mathbf{u}, U, \cdot) \geq 2$ in a neighbourhood of $\mathbf{y}$. On the other hand, since $U \in \mathcal{U}_{\mathbf{u}}^{\text {in }}$, we have that $\mathcal{N}_{U}=1$ a.e. in $\operatorname{im}_{\mathrm{G}}(\mathbf{u}, U)$, so by $(4.18)$ we obtain that $\operatorname{deg}(\mathbf{u}, U, \cdot)=1$ a.e. $\operatorname{in}_{\operatorname{im}_{\mathrm{T}}}(\mathbf{u}, U)$, which is a contradiction.

Part b). Call $\mathbf{y}:=\hat{\mathbf{u}}(\mathbf{x})$. Clearly, $\mathbf{x} \in G_{U}(\mathbf{y})$. Assume, for a contradiction, that $G_{U}(\mathbf{y})$ has more than one point. Since it is connected by part $a$ ), for every sufficiently small $r>0$ there exists $\mathbf{x}_{r} \in G_{U}(\mathbf{y}) \cap \partial B(\mathbf{x}, r)$. Taking only those $r>0$ such that $\partial B(\mathbf{x}, r) \cap N C=\varnothing$, we find that for a.e. $r>0$ sufficiently small there exists $\mathbf{x}_{r} \in \partial B(\mathbf{x}, r)$ such that $\mathbf{y}=\hat{\mathbf{u}}\left(\mathbf{x}_{r}\right)$. This is a contradiction with Lemma 3.2, according to which $\mathbf{y} \notin \hat{\mathbf{u}}(\partial B(\mathbf{x}, h))$ for all $h$ in a set $A \subset(0, \infty)$ of density $\frac{1}{2}$ at 0 .

Part c). Since $G_{U}(T) \cap U_{+} \backslash N C=\varnothing$ due to part b), we have $\mathcal{L}^{n}\left(G_{U}(T)\right)=0$ thanks to Proposition 5.9 . It remains to show that $\mathcal{H}^{n-1}(T)=0$. Assume that $T \neq \varnothing$. Denote by $\pi^{i}: \mathbb{R}^{n} \rightarrow \mathbb{R}$ the projection onto the $i$ th coordinate, $i \in\{1, \ldots, n\}$, and by $H_{t}^{i}$ the hyperplane $\left\{\mathbf{x} \in \mathbb{R}^{n}: \pi^{i}(\mathbf{x})=t\right\}, t \in \mathbb{R}$. We also denote by $\mathcal{J}^{i}$ the family of non-empty open intervals with rational endpoints that are included in $\pi^{i}\left(G_{U}(\mathbf{y})\right)$ for some $\mathbf{y} \in T$. For every $\mathbf{y} \in T$ the set $G_{U}(\mathbf{y})$ is connected by part a) and contains more than one point. Therefore, for at least one $i \in\{1, \ldots, n\}$ (depending on $\mathbf{y}$ ) there exists a $J \in \mathcal{J}^{i}$ such that $J \subset \pi^{i}\left(G_{U}(\mathbf{y})\right.$ ). Given $i \in\{1, \ldots, n\}$ and $J \in \mathcal{J}^{i}$, we set $T_{J}^{i}:=\left\{\mathbf{y} \in T: J \subset \pi^{i}\left(G_{U}(\mathbf{y})\right)\right\}$.

Let $i \in\{1, \ldots, n\}$ and $J \in \mathcal{J}^{i}$. Bearing in mind that $\mathcal{H}^{\max \{n-p, 0\}}(N C)=0$ and that $\mathcal{L}^{n}\left(G_{U}(T)\right)=0$, for a.e. $t \in J$ the intersection $H_{t}^{i} \cap N C$ is empty, the intersection $H_{t}^{i} \cap G_{U}\left(T_{J}^{i}\right)$ has null $\mathcal{H}^{n-1}$ measure and $\mathbf{u} \in W^{1, p}\left(H_{t}^{i} \cap \Omega, \mathbb{R}^{n}\right)$. Fix a $t_{J}$ in $J$ such that

$$
H_{t_{J}}^{i} \cap N C=\varnothing, \quad \mathcal{H}^{n-1}\left(H_{t_{J}}^{i} \cap G_{U}\left(T_{J}^{i}\right)\right)=0, \quad \text { and } \mathbf{u} \in W^{1, p}\left(H_{t_{J}}^{i} \cap \Omega, \mathbb{R}^{n}\right) .
$$

We claim that

$$
T \subset \bigcup_{i=1}^{n} \bigcup_{J \in \mathcal{J}^{i}} \mathbf{u}\left(H_{t_{J}}^{i} \cap G_{U}\left(T_{J}^{i}\right)\right) .
$$

Indeed, given $\mathbf{y} \in T$, let $i \in\{1, \ldots, n\}$ and $J \in \mathcal{J}^{i}$ be such that $J \subset \pi^{i}\left(G_{U}(\mathbf{y})\right)$, and let $\mathbf{x} \in H_{t_{J}}^{i} \cap G_{U}(\mathbf{y})$. Since $\mathbf{x} \notin N C$ we have $\mathbf{u}(\mathbf{x})=\mathbf{y}$. Moreover, since $\mathbf{y} \in T_{J}^{i}$, it follows that $\mathbf{x} \in G_{U}\left(T_{J}^{i}\right)$ so $\mathbf{y}=\mathbf{u}(\mathbf{x}) \in$ $\mathbf{u}\left(H_{t_{J}}^{i} \cap G_{U}\left(T_{J}^{i}\right)\right)$. Inclusion (5.7) is then proved. Finally, by Remark $\left.2.16 \mathrm{~b}\right), \mathcal{H}^{n-1}\left(\mathbf{u}\left(H_{t_{J}}^{i} \cap G_{U}\left(T_{J}^{i}\right)\right)\right)=0$. From (5.7) we conclude that $\mathcal{H}^{n-1}(T)=0$.

Part d). As $\mathbf{u}$ is one-to-one a.e. in $U$, we have $\mathcal{N}_{U}=1$ a.e. in $\operatorname{im}_{\mathrm{G}}(\mathbf{u}, U)$ and, due to (5.1), there exists a set $N \subset \operatorname{im}_{\mathrm{T}}(\mathbf{u}, U)$ of zero measure such that $\mathcal{N}_{U}=1 \mathrm{in} \operatorname{im}_{\mathrm{T}}(\mathbf{u}, U) \backslash N$. By Proposition 5.9, the set $M:=\mathbf{u}\left(U \backslash U_{+}\right)$has zero measure. Then, by part $\left.b\right)$, for each $\mathbf{y} \in \operatorname{im}_{\mathrm{T}}(\mathbf{u}, U) \backslash(N \cup M)$ there exists $\mathbf{x} \in U_{+}$ such that $G_{U}(\mathbf{y})=\{\mathbf{x}\}$. Therefore $T^{\prime} \subset N \cup M$.

The sets $T$ and $T^{\prime}$ are singular regions in the deformed configuration. While $T^{\prime}$ represents damage zones in $\operatorname{im}_{\mathrm{T}}(\mathbf{u}, U)$, the set $T$ indicates a degeneration of the material coming from $G_{U}(T)$ due to extreme compression, with a loss of the injectivity.

We can now define a precise representative of the inverse of Definition 5.2.

Definition 5.15. Let $\mathbf{u} \in \mathcal{A}_{p}$ and $U \in \mathcal{U}_{\mathbf{u}}^{N \text {,in }}$. Define $\hat{\mathbf{u}}_{U}^{-1}: \operatorname{im}_{\mathrm{T}}(\mathbf{u}, U) \rightarrow \mathbb{R}^{n}$ as

$$
\hat{\mathbf{u}}_{U}^{-1}(\mathbf{y})=\text { any element of } G_{U}(\mathbf{y})
$$


By Lemma 5.13 e) and the axiom of choice, $\hat{\mathbf{u}}_{U}^{-1}$ is well defined. Moreover, by Lemma 5.13 a), it takes values in $U$. Furthermore, having in mind Proposition $5.14 \mathrm{c}$ ) and the set $T$ defined therein, for each $\mathbf{y} \in \operatorname{im}_{\mathrm{T}}(\mathbf{u}, U) \backslash T$ there is a unique $\mathbf{x} \in U$ such that $\mathbf{y} \in \operatorname{im}_{\mathrm{T}}(\mathbf{u}, \mathbf{x})$, hence the definition of $\hat{\mathbf{u}}_{U}^{-1}$ is univocal up to an $\mathcal{H}^{n-1}$-negligible set. In particular, as $\hat{\mathbf{u}}(\mathbf{x}) \in \operatorname{im}_{\mathrm{T}}(\mathbf{u}, \mathbf{x})$ for all $\mathbf{x} \in \Omega$, we have that $\hat{\mathbf{u}}_{U}^{-1}=\left(\left.\hat{\mathbf{u}}\right|_{U}\right)^{-1}$ in $\operatorname{im}_{\mathrm{G}}(\hat{\mathbf{u}}, U) \cap \operatorname{im}_{\mathrm{T}}(\mathbf{u}, U) \backslash T$, where $\left(\left.\hat{\mathbf{u}}\right|_{U}\right)^{-1}$ is as in Definition 5.2. Of course, $\operatorname{im}_{\mathrm{G}}(\hat{\mathbf{u}}, U) \cap \operatorname{im}_{\mathrm{T}}(\mathbf{u}, U) \backslash T$ is of full measure in $\operatorname{im}_{\mathrm{T}}(\mathbf{u}, U)$, thanks to (4.1). In fact, it is easy to verify (see, if necessary, Lemma 7.2 below and Remark 2.21) that $\hat{\mathbf{u}}_{U}^{-1}=\left(\left.\mathbf{u}\right|_{U}\right)^{-1}$ a.e. in $\operatorname{im}_{\mathrm{T}}(\mathbf{u}, U)$, for any representative $\mathbf{u}$.

The function $\hat{\mathbf{u}}_{U}^{-1}$ is an inverse of $\hat{\mathbf{u}}$ in the following sense: considering the set $T^{\prime}$ of Proposition $5.14 d$ ), we have that $\hat{\mathbf{u}}\left(\hat{\mathbf{u}}_{U}^{-1}(\mathbf{y})\right)=\mathbf{y}$ for each $\mathbf{y} \in \operatorname{im}_{\mathrm{T}}(\mathbf{u}, U) \backslash T^{\prime}$, while $\hat{\mathbf{u}}_{U}^{-1}(\hat{\mathbf{u}}(\mathbf{x}))=\mathbf{x}$ for each $\mathbf{x} \in \operatorname{im}_{\mathrm{T}}(\mathbf{u}, U)^{*} \cap U \backslash$ $G_{U}(T)$. Note that, since $\operatorname{im}_{\mathrm{T}}(\mathbf{u}, U)^{*} \cap U=U \backslash G_{U}(\mathbf{u}(\partial U))$ for $U \in \mathcal{U}_{\mathbf{u}}^{N}$, one has $\operatorname{im}_{\mathrm{T}}(\mathbf{u}, U)^{*} \cap U \backslash G_{U}(T)=$ $U \backslash G_{U}(T \cup \mathbf{u}(\partial U))$.

The main result of this section is the continuity of $\hat{\mathbf{u}}_{U}^{-1}$ at $\mathcal{H}^{n-1}$-a.e. point.

Proposition 5.16. Let $\mathbf{u} \in \mathcal{A}_{p}$ and $U \in \mathcal{U}_{\mathbf{u}}^{N \text {,in }}$, and consider the set $T$ of Proposition 5.14c). Then $\hat{\mathbf{u}}_{U}^{-1}$ is continuous at each point of $\operatorname{im}_{\mathrm{T}}(\mathbf{u}, U) \backslash T$.

Proof. Given $\mathbf{y} \in \operatorname{im}_{\mathrm{T}}(\mathbf{u}, U) \backslash T$, let $\mathbf{x}:=\hat{\mathbf{u}}_{U}^{-1}(\mathbf{y})$. Since $\mathbf{y} \notin T$, given $r>0$ such that $\bar{B}(\mathbf{x}, r) \subset U$ and $\partial B(\mathbf{x}, r) \cap N C=\varnothing$, it is not possible that $\mathbf{y} \in \hat{\mathbf{u}}(\partial B(\mathbf{x}, r))$. In particular, by Lemma 5.4,

$$
\mathbf{y} \in \operatorname{im}_{\mathrm{T}}(\mathbf{u}, \mathbf{x})=\bigcap_{\substack{r>0 \\ B(\mathbf{x}, r) \in \mathcal{U}_{\mathbf{u}}^{N}}} \operatorname{im}_{\mathrm{T}}(\mathbf{u}, B(\mathbf{x}, r)) .
$$

We shall show that for any $r>0$ there exists a neighbourhood $A$ of $\mathbf{y}$ such that if $\mathbf{y}^{\prime} \in A$ then $\hat{\mathbf{u}}_{U}^{-1}\left(\mathbf{y}^{\prime}\right) \in$ $B(\mathbf{x}, r)$. Let $r^{\prime}<r$ be such that $B\left(\mathbf{x}, r^{\prime}\right) \in \mathcal{U}_{\mathbf{u}}^{N}$ and let $A:=\operatorname{im}_{\mathrm{T}}\left(\mathbf{u}, B\left(\mathbf{x}, r^{\prime}\right)\right) \cap \operatorname{im}_{\mathrm{T}}(\mathbf{u}, U)$. If $\mathbf{y}^{\prime} \in A$, then $G_{U}\left(\mathbf{y}^{\prime}\right) \cap \partial B\left(\mathbf{x}, r^{\prime}\right)=\varnothing$. Therefore, since $G_{U}\left(\mathbf{y}^{\prime}\right)$ is connected by Proposition $\left.5.14 a\right)$, we have two alternatives: $G_{U}\left(\mathbf{y}^{\prime}\right) \subset B\left(\mathbf{x}, r^{\prime}\right)$ or $G_{U}\left(\mathbf{y}^{\prime}\right) \cap \bar{B}\left(\mathbf{x}, r^{\prime}\right)=\varnothing$. Suppose, for a contradiction, that the second option holds. Since, by Lemma $5.13, G_{U}\left(\mathbf{y}^{\prime}\right)$ is compact and contained in $U$, we can find a $U^{\prime} \in \mathcal{U}_{\mathbf{u}}^{N}$ contained in $U$ such that $\bar{U}^{\prime} \cap \bar{B}\left(\mathbf{x}, r^{\prime}\right)=\varnothing$ and $G_{U}\left(\mathbf{y}^{\prime}\right) \subset U^{\prime}$. This last inclusion implies that $\mathbf{y}^{\prime} \notin \hat{\mathbf{u}}\left(\partial U^{\prime}\right)$. Let $\mathbf{x}^{\prime} \in G_{U}\left(\mathbf{y}^{\prime}\right)$; then $\mathbf{x}^{\prime} \in U^{\prime}$ and, by Proposition $\left.4.3 d\right)$ and Lemma 5.4,

$$
\mathbf{y}^{\prime} \in \operatorname{im}_{\mathrm{T}}\left(\mathbf{u}, \mathbf{x}^{\prime}\right) \subset \operatorname{im}_{\mathrm{T}}\left(\mathbf{u}, U^{\prime}\right) \text {. }
$$

Thus, by Proposition $4.3 c), \operatorname{deg}\left(\mathbf{u}, U^{\prime}, \mathbf{y}^{\prime}\right) \geq 1$ and $\operatorname{deg}\left(\mathbf{u}, B\left(\mathbf{x}, r^{\prime}\right), \mathbf{y}^{\prime}\right) \geq 1$. By the additivity of the degree, we find that

$$
2 \leq \operatorname{deg}\left(\mathbf{u}, U^{\prime}, \mathbf{y}^{\prime}\right)+\operatorname{deg}\left(\mathbf{u}, B\left(\mathbf{x}, r^{\prime}\right), \mathbf{y}^{\prime}\right)=\operatorname{deg}\left(\mathbf{u}, U^{\prime} \cup B\left(\mathbf{x}, r^{\prime}\right), \mathbf{y}^{\prime}\right) .
$$

In particular, $\operatorname{deg}\left(\mathbf{u}, U^{\prime} \cup B\left(\mathbf{x}, r^{\prime}\right), \cdot\right) \geq 2$ in a non-empty open set. By Lemma $2.22, U^{\prime} \cup B\left(\mathbf{x}, r^{\prime}\right) \in \mathcal{U}_{\mathbf{u}}^{\text {in }}$, so, by (4.1), $\operatorname{deg}\left(\mathbf{u}, U^{\prime} \cup B\left(\mathbf{x}, r^{\prime}\right), \cdot\right) \leq 1$ a.e., a contradiction.

Therefore, $G_{U}\left(\mathbf{y}^{\prime}\right) \subset B\left(\mathbf{x}, r^{\prime}\right)$ and, consequently, $\hat{\mathbf{u}}_{U}^{-1}\left(\mathbf{y}^{\prime}\right) \in B(\mathbf{x}, r)$.

\subsection{The topological image}

Since $\Omega \notin \mathcal{U}_{\mathbf{u}}$, a suitable definition of the topological image $\operatorname{im}_{\mathrm{T}}(\mathbf{u}, \Omega)$ of $\Omega$ under $\mathbf{u}$ must be given. In this subsection we explore its properties, which will be essential in the applications of the next sections.

Definition 5.17. Let $\mathbf{u} \in \mathcal{A}_{p}$. Define

$$
\operatorname{im}_{\mathrm{T}}(\mathbf{u}, \Omega):=\bigcup_{U \in \mathcal{U}_{\mathbf{u}}^{N}} \operatorname{im}_{\mathrm{T}}(\mathbf{u}, U)
$$

We will see in Section 8 that $\operatorname{im}_{\mathrm{T}}(\mathbf{u}, \Omega)$ plays the role of the deformed configuration. By the continuity of the degree, $\operatorname{im}_{\mathrm{T}}(\mathbf{u}, U)$ is open, and hence, so is $\operatorname{im}_{\mathrm{T}}(\mathbf{u}, \Omega)$. Notice also that, owing to the Lindelöf property, there exists a countable family $\mathcal{B} \subset \mathcal{U}_{\mathbf{u}}^{N}$ such that

$$
\operatorname{im}_{\mathrm{T}}(\mathbf{u}, \Omega)=\bigcup_{B \in \mathcal{B}} \operatorname{im}_{\mathrm{T}}(\mathbf{u}, B) .
$$


We present some properties of the topological image. Recall the notation $U_{t}$ of $(2.2)$.

Lemma 5.18. Let $\mathbf{u} \in \mathcal{A}_{p}$. The following properties hold:

a) For each $U \in \mathcal{U}_{\mathbf{u}}^{N}$ and each compact $K \subset \mathrm{im}_{\mathrm{T}}(\mathbf{u}, U)$ there exists $\delta>0$ such that

$$
K \subset \bigcap_{\substack{t \in(0, \delta) \\ U_{t} \in \mathcal{U}_{\mathbf{u}}^{N}}} \operatorname{im}_{\mathrm{T}}\left(\mathbf{u}, U_{t}\right) .
$$

b) If $\mathbf{u}_{1} \in \mathcal{A}_{p}$ and $\mathbf{u}_{1}=\mathbf{u}$ a.e. then $\operatorname{im}_{\mathrm{T}}(\mathbf{u}, \Omega)=\operatorname{im}_{\mathrm{T}}\left(\mathbf{u}_{1}, \Omega\right)$.

c) $\operatorname{im}_{\mathrm{T}}(\mathbf{u}, \Omega)=\operatorname{im}_{\mathrm{G}}(\mathbf{u}, \Omega)$ a.e.

d) There exists a disjoint family $\left\{V_{k}\right\}_{k \in \mathbb{N}}$ of open sets such that

$$
\operatorname{im}_{\mathrm{T}}(\mathbf{u}, \Omega)=\bigcup_{k \in \mathbb{N}} V_{k} \quad \text { a.e. }
$$

and for each $k \in \mathbb{N}$ there exists $B_{k} \in \mathcal{U}_{\mathbf{u}}^{N}$ for which $V_{k} \subset \subset \operatorname{im}_{\mathrm{T}}\left(\mathbf{u}, B_{k}\right)$.

Proof. Part a). By Remarks 2.21 and 5.10 we have $\left.\mathbf{u}^{*}\right|_{\partial U}=\left.\hat{\mathbf{u}}\right|_{\partial U}, U \in \mathcal{U}_{\hat{\mathbf{u}}}$, the continuous representative of $\left.\mathbf{u}\right|_{\partial U}$ is $\left.\hat{\mathbf{u}}\right|_{\partial U}$, and, due to Convention 2.14, $\left.\mathbf{u}\right|_{\partial U}=\left.\hat{\mathbf{u}}\right|_{\partial U}$. Consider $\eta:=\operatorname{dist}(K, \hat{\mathbf{u}}(\partial U))$. As $\hat{\mathbf{u}}$ is continuous at each point of the compact set $\partial U$, an elementary property shows that there exists $\delta>0$ such that if $\mathbf{x}_{0} \in \partial U$ and $\mathbf{x} \in B\left(\mathbf{x}_{0}, \delta\right)$ then $\mathbf{x} \in \Omega$ and

$$
\left|\hat{\mathbf{u}}(\mathbf{x})-\hat{\mathbf{u}}\left(\mathbf{x}_{0}\right)\right|<\eta .
$$

Before continuing with the proof, we establish some results and notation that were inherent in the construction of Section 2.4; we refer to [27, Th. 16.25.2] for the proofs. Making the above $\delta>0$ smaller, we can assume that the map

$$
\begin{aligned}
\mathbf{p}: \partial U \times(-\delta, \delta) & \rightarrow\left\{\mathbf{z} \in \mathbb{R}^{n}: \operatorname{dist}(\mathbf{z}, \partial U)<\delta\right\} \\
\left(\mathbf{x}_{0}, t\right) & \mapsto \mathbf{x}_{0}-t \boldsymbol{\nu}\left(\mathbf{x}_{0}\right)
\end{aligned}
$$

is a $C^{1}$ diffeomorphism, where $\boldsymbol{\nu}$ is the unit exterior normal to $\partial U$.

Take any $t \in(0, \delta)$ such that $U_{t} \in \mathcal{U}_{\mathbf{u}}^{N}$. Then the continuous representative of $\left.\mathbf{u}\right|_{\partial U_{t}}$ is $\left.\hat{\mathbf{u}}\right|_{\partial U_{t}}$. Define $\mathbf{v}: \bar{U} \rightarrow \mathbb{R}^{n}$ as

$$
\begin{cases}\mathbf{v}(\mathbf{x}):=\mathbf{u}(\mathbf{x}) & \text { if } \mathbf{x} \in U_{t} \\ \mathbf{v}\left(\mathbf{p}\left(\mathbf{x}_{0}, \lambda\right)\right):=\left(1-\frac{\lambda}{t}\right) \hat{\mathbf{u}}\left(\mathbf{x}_{0}\right)+\frac{\lambda}{t} \hat{\mathbf{u}}\left(\mathbf{p}\left(\mathbf{x}_{0}, t\right)\right) & \text { if }\left(\mathbf{x}_{0}, \lambda\right) \in \partial U \times[0, t] .\end{cases}
$$

Note that $\mathbf{v}$ is defined a.e. in $U_{t}$ and everywhere in $\bar{U} \backslash U_{t}$; in fact, $\left.\mathbf{v}\right|_{\bar{U} \backslash U_{t}}$ is continuous,

$$
\mathbf{p}(\partial U \times[0, t])=\bar{U} \backslash U_{t} \quad \text { and } \quad \mathbf{p}(\partial U \times\{t\})=\partial U_{t} .
$$

By construction, $\mathbf{v}=\hat{\mathbf{u}}$ in $\partial U \cup \partial U_{t}$ so

$$
\operatorname{deg}(\mathbf{v}, U, \cdot)=\operatorname{deg}(\mathbf{u}, U, \cdot) \text { in } \mathbb{R}^{n} \backslash \mathbf{u}(\partial U) \quad \text { and } \quad \operatorname{deg}\left(\mathbf{v}, U_{t}, \cdot\right)=\operatorname{deg}\left(\mathbf{u}, U_{t}, \cdot\right) \text { in } \mathbb{R}^{n} \backslash \mathbf{u}\left(\partial U_{t}\right) .
$$

Now, thanks to (5.9) we have that for each $\mathbf{y} \in K, \mathbf{x}_{0} \in \partial U$ and $\lambda \in[0, t]$,

$$
\left|\mathbf{y}-\mathbf{v}\left(\mathbf{p}\left(\mathbf{x}_{0}, \lambda\right)\right)\right| \geq\left|\mathbf{y}-\hat{\mathbf{u}}\left(\mathbf{x}_{0}\right)\right|-\frac{\lambda}{t}\left|\hat{\mathbf{u}}\left(\mathbf{x}_{0}\right)-\hat{\mathbf{u}}\left(\mathbf{p}\left(\mathbf{x}_{0}, t\right)\right)\right|>0,
$$

so $\mathbf{y} \notin \mathbf{v}\left(\bar{U} \backslash U_{t}\right)$. By the excision property of the degree, $\operatorname{deg}(\mathbf{v}, U, \mathbf{y})=\operatorname{deg}\left(\mathbf{v}, U_{t}, \mathbf{y}\right)$ so, in total,

$$
0 \neq \operatorname{deg}(\mathbf{u}, U, \mathbf{y})=\operatorname{deg}(\mathbf{v}, U, \mathbf{y})=\operatorname{deg}\left(\mathbf{v}, U_{t}, \mathbf{y}\right)=\operatorname{deg}\left(\mathbf{u}, U_{t}, \mathbf{y}\right),
$$


and, hence $\mathbf{y} \in \operatorname{im}_{\mathrm{T}}\left(\mathbf{u}, U_{t}\right)$.

Part b). Let $\mathbf{y} \in \operatorname{im}_{\mathrm{T}}(\mathbf{u}, \Omega)$ and let $U \in \mathcal{U}_{\mathbf{u}}^{N}$ be such that $\mathbf{y} \in \operatorname{im}_{\mathrm{T}}(\mathbf{u}, U)$. By part a) and Lemma 5.12, there exists $t>0$ such that $U_{t} \in \mathcal{U}_{\mathbf{u}}^{N} \cap \mathcal{U}_{\mathbf{u}_{1}}^{N}$ and $\mathbf{y} \in \operatorname{im}_{\mathrm{T}}\left(\mathbf{u}, U_{t}\right)$. By Remark 2.19, $\operatorname{im}_{\mathrm{T}}\left(\mathbf{u}, U_{t}\right)=\operatorname{im}_{\mathrm{T}}\left(\mathbf{u}_{1}, U_{t}\right)$, so $\mathbf{y} \in \operatorname{im}_{\mathrm{T}}\left(\mathbf{u}_{1}, \Omega\right)$. The symmetry of the argument provides the other inclusion.

Part c). By Lemma 5.12, there exists an increasing sequence $\left\{U_{k}\right\}_{k \in \mathbb{N}}$ in $\mathcal{U}_{\mathbf{u}}^{N}$ such that $\Omega=\bigcup_{k \in \mathbb{N}} U_{k}$, so $\operatorname{im}_{\mathrm{G}}(\mathbf{u}, \Omega)=\bigcup_{k \in \mathbb{N}} \operatorname{im}_{\mathrm{G}}\left(\mathbf{u}, U_{k}\right)$. Let $\mathcal{B}$ be the family of (5.8). Since for any $B \in \mathcal{B}$ there exists $k \in \mathbb{N}$ such that $B \subset \subset U_{k}$, we have, by Proposition $\left.4.3 d\right)$, that $\operatorname{im}_{\mathrm{T}}(\mathbf{u}, B) \subset \operatorname{im}_{\mathrm{T}}\left(\mathbf{u}, U_{k}\right) \subset \operatorname{im}_{\mathrm{T}}(\mathbf{u}, \Omega)$ a.e. Therefore, $\operatorname{im}_{\mathrm{T}}(\mathbf{u}, \Omega)=\bigcup_{k \in \mathbb{N}} \operatorname{im}_{\mathrm{T}}\left(\mathbf{u}, U_{k}\right)$ a.e. Using now (5.1), the above equalities may be summarized as

$$
\operatorname{im}_{\mathrm{T}}(\mathbf{u}, \Omega)=\bigcup_{k \in \mathbb{N}} \operatorname{im}_{\mathrm{T}}\left(\mathbf{u}, U_{k}\right)=\bigcup_{k \in \mathbb{N}} \operatorname{im}_{\mathrm{G}}\left(\mathbf{u}, U_{k}\right)=\operatorname{im}_{\mathrm{G}}(\mathbf{u}, \Omega) \quad \text { a.e. }
$$

Part d). For each $U \in \mathcal{U}_{\mathbf{u}}^{N}$ let $\mathcal{V}_{U}$ be the family of all open sets $V$ such that $V \subset \subset \operatorname{im}_{\mathrm{T}}(\mathbf{u}, U)$. The conclusion then follows from a standard application of Besicovitch's covering theorem to the family $\{V: V \in$ $\mathcal{V}_{U}$ for some $\left.U \in \mathcal{U}_{\mathbf{u}}^{N}\right\}$.

\section{Stability of the inverse}

In this section we show an important stability result in $\mathcal{A}_{p}$ : the weak $W^{1, p}$-convergence of a sequence in $\mathcal{A}_{p}$ implies the weak $W^{1,1}$-convergence of the local inverses (Theorem 6.3). We first state a compactness property of $\mathcal{A}_{p}$, which is a direct consequence of the main result of [42].

Proposition 6.1. Let $p>n-1$ and, for each $j \in \mathbb{N}$ let $\mathbf{u}_{j} \in \mathcal{A}_{p}$. Assume that $\left\{\mathbf{u}_{j}\right\}_{j \in \mathbb{N}}$ is bounded in $W^{1, p}\left(\Omega, \mathbb{R}^{n}\right)$ and $\left\{\operatorname{det} D \mathbf{u}_{j}\right\}_{j \in \mathbb{N}}$ is equiintegrable. Then there exists $\mathbf{u} \in W^{1, p}\left(\Omega, \mathbb{R}^{n}\right)$ with $\operatorname{det} D \mathbf{u} \geq 0$ a.e., $\operatorname{det} D \mathbf{u} \in L^{1}(\Omega)$ and $\mathcal{E}(\mathbf{u})=0$ such that, for a subsequence,

$$
\mathbf{u}_{j} \rightarrow \mathbf{u} \text { in } W^{1, p}\left(\Omega, \mathbb{R}^{n}\right) \quad \text { and } \quad \operatorname{det} D \mathbf{u}_{j} \rightarrow \operatorname{det} D \mathbf{u} \text { in } L^{1}(\Omega)
$$

as $j \rightarrow \infty$. Moreover, if $\operatorname{det} D \mathbf{u}>0$ a.e. then $\mathbf{u} \in \mathcal{A}_{p}$.

Proof. For a subsequence (not relabelled), we have that there exist $\mathbf{u} \in W^{1, p}\left(\Omega, \mathbb{R}^{n}\right)$ and $\theta \in L^{1}(\Omega)$ such that

$$
\mathbf{u}_{j} \rightarrow \mathbf{u} \text { in } W^{1, p}\left(\Omega, \mathbb{R}^{n}\right) \text { and } \operatorname{det} D \mathbf{u}_{j} \rightarrow \theta \text { in } L^{1}(\Omega) .
$$

as $j \rightarrow \infty$. Thanks to [42, Th. 3], we have $\theta=\operatorname{det} D \mathbf{u}$ a.e. and $\mathcal{E}(\mathbf{u})=0$. Clearly, $\operatorname{det} D \mathbf{u} \geq 0$ a.e., and if $\operatorname{det} D \mathbf{u}>0$ a.e. then $\mathbf{u} \in \mathcal{A}_{p}$.

As mentioned after Definition 4.8, [59, 38] also worked with the class $\mathcal{A}_{p}$ and, in fact, Proposition 6.1 is also a direct consequence of [59, Th. 4] or else [38, Th. 3.3.2.1 and Prop. 3.3.2.3].

Remark 6.2. The equiintegrability of $\left\{\operatorname{det} D \mathbf{u}_{j}\right\}_{j \in \mathbb{N}}$ cannot be removed from the statement of Proposition 6.1. Without that assumption, the weak limit in $W^{1, p}$ of a sequence in $\mathcal{A}_{p}$ may not be in $\mathcal{A}_{p}$, even if the limit function $\mathbf{u}$ satisfies $\operatorname{det} D \mathbf{u}>0$ a.e. In fact, Ponomarev's [67] example (see also [61, Sect. 11]) exhibits such a situation. It was shown that, when $\Omega$ is the unit square in $\mathbb{R}^{2}$, there exist a sequence $\left\{\mathbf{u}_{j}\right\}_{j \in \mathbb{N}}$ of Lipschitz homeomorphisms and a homeomorphism $\mathbf{u}$ in $W^{1, p}\left(\Omega, \mathbb{R}^{2}\right)$, for every $p<2$, such that $\mathbf{u}_{j} \rightarrow \mathbf{u}$ in $W^{1, p}\left(\Omega, \mathbb{R}^{2}\right)$ as $j \rightarrow \infty$. Moreover, $\operatorname{det} D \mathbf{u}_{j}>0$ a.e. and $\left\|\operatorname{det} D \mathbf{u}_{j}\right\|_{L^{1}\left(\Omega, \mathbb{R}^{2}\right)}=1$ for each $j \in \mathbb{N}$, whereas $\operatorname{det} D \mathbf{u}>0$ a.e. and $\operatorname{det} D \mathbf{u} \in L^{1}\left(\Omega, \mathbb{R}^{2}\right)$. As each $\mathbf{u}_{j}$ is Lipschitz, it follows that $\mathcal{E}\left(\mathbf{u}_{j}\right)=0$. The same reasoning that in [61] showed that $\operatorname{Per}_{\mathrm{G}}(\mathbf{u}, \Omega)=\infty$ can be adapted to show that $\mathcal{E}(\mathbf{u})=\infty$; in fact, a shorter argument goes as follows. The deformation $\mathbf{u}$ does not satisfy Lusin's $N$ condition, as shown in [61]. By [42, Th. $5.5]$, a homeomorphism in $W^{1, p}\left(\Omega, \mathbb{R}^{2}\right)$ not satisfying Lusin's $N$ condition has infinite surface energy $\mathcal{E}$. In conclusion, $\mathbf{u}_{j} \in \mathcal{A}_{p}$ for all $j \in \mathbb{N}$ but $\mathbf{u} \notin \mathcal{A}_{p}$. 
The main result of this section shows that the ball of local invertibility of a $\mathbf{u} \in \mathcal{A}_{p}$ (see Proposition $4.5 d$ ) or Corollary 4.7) can be chosen to be the same for all members of a $W^{1, p}$-weakly convergent sequence to $\mathbf{u}$, and proves the convergence of the derivative of the inverse and its minors in the appropriate topology. It generalizes the result contained in [32, Lemmas 4.3 and 4.5]. Of course, a minor refers to a subdeterminant defined in the set $\mathbb{R}^{n \times n}$ of matrices.

Theorem 6.3. For each $j \in \mathbb{N}$, let $\mathbf{u}_{j}, \mathbf{u} \in \mathcal{A}_{p}$ satisfy $\mathbf{u}_{j} \rightarrow \mathbf{u}$ in $W^{1, p}\left(\Omega, \mathbb{R}^{n}\right)$ as $j \rightarrow \infty$. The following assertions hold:

a) For any $U \in \mathcal{U}_{\mathbf{u}}^{N}$ and any compact set $K \subset \operatorname{im}_{\mathrm{T}}(\mathbf{u}, U)$ there exists a subsequence for which $K \subset \operatorname{im}_{\mathrm{T}}\left(\mathbf{u}_{j}, \Omega\right)$ for all $j \in \mathbb{N}$.

b) For a subsequence, there exists a disjoint family

$$
\left\{B_{k}\right\}_{k \in \mathbb{N}} \subset \mathcal{U}_{\mathbf{u}}^{N \text {,in }} \cap \bigcap_{j \in \mathbb{N}} \mathcal{U}_{\mathbf{u}_{j}}^{N, \text { in }}
$$

such that $\Omega=\bigcup_{k \in \mathbb{N}} B_{k}$ a.e. and, for each $k \in \mathbb{N}$,

$$
\mathbf{u}_{j} \rightarrow \mathbf{u} \quad \text { uniformly in } \partial B_{k} \text { as } j \rightarrow \infty .
$$

c) Let $B \in \mathcal{U}_{\mathbf{u}}^{\mathrm{in}} \cap \bigcap_{j \in \mathbb{N}} \mathcal{U}_{\mathbf{u}_{j}}^{\mathrm{in}}$ and take an open set $V \subset \subset \operatorname{im}_{\mathrm{T}}(\mathbf{u}, B)$ such that $V \subset \operatorname{im}_{\mathrm{T}}\left(\mathbf{u}_{j}, B\right)$ for all $j \in \mathbb{N}$. Then

1) $\left(\left.\mathbf{u}_{j}\right|_{B}\right)^{-1} \stackrel{*}{\rightarrow}\left(\left.\mathbf{u}\right|_{B}\right)^{-1}$ in $B V\left(V, \mathbb{R}^{n}\right)$ as $j \rightarrow \infty$;

2) for any minor $M$, we have $M\left(D\left(\left.\mathbf{u}_{j}\right|_{B}\right)^{-1}\right), M\left(D\left(\left.\mathbf{u}\right|_{B}\right)^{-1}\right) \in L^{1}(V)$ for all $j \in \mathbb{N}$ and

$$
M\left(D\left(\left.\mathbf{u}_{j}\right|_{B}\right)^{-1}\right) \stackrel{*}{\rightarrow} M\left(D\left(\left.\mathbf{u}\right|_{B}\right)^{-1}\right) \quad \text { in } \mathcal{M}(V) \text { as } j \rightarrow \infty .
$$

If, in addition, the sequence $\left\{\operatorname{det} D\left(\left.\mathbf{u}_{j}\right|_{B}\right)^{-1}\right\}_{j \in \mathbb{N}}$ is equiintegrable in $V$, then the convergence in c1) holds in the weak topology of $W^{1,1}\left(V, \mathbb{R}^{n}\right)$, and the convergence in c2) holds in the weak topology of $L^{1}(V)$.

d) For a subsequence we have that $\chi_{\mathrm{im}_{\mathrm{T}}\left(\mathbf{u}_{j}, \Omega\right)} \rightarrow \chi_{\mathrm{im}_{\mathrm{T}}(\mathbf{u}, \Omega)}$ a.e. and in $L^{1}\left(\mathbb{R}^{n}\right)$ as $j \rightarrow \infty$.

Proof. Part a). By Lemmas 5.18a), 5.12 and 2.24 there exists $t>0$ such that, $U_{t} \in \mathcal{U}_{\mathbf{u}}^{N} \cap \bigcap_{j \in \mathbb{N}} \mathcal{U}_{\mathbf{u}_{j}}^{N}$, $K \subset \operatorname{im}_{\mathrm{T}}\left(\mathbf{u}, U_{t}\right)$ and, for a subsequence,

$$
\mathbf{u}_{j} \rightarrow \mathbf{u} \quad \text { uniformly in } \partial U_{t} \text { as } j \rightarrow \infty \text {. }
$$

Thanks to Lemma 3.6, there exists $j_{K} \in \mathbb{N}$ such that $K \subset \operatorname{im}_{\mathrm{T}}\left(\mathbf{u}_{j}, U_{t}\right) \subset \operatorname{im}_{\mathrm{T}}\left(\mathbf{u}_{j}, \Omega\right)$ for all $j \geq j_{K}$. The conclusion follows.

Part b). First we prove that for a.e. $\mathbf{x}_{0} \in \Omega$ there exist a subsequence (not relabelled) and $U_{\mathbf{x}_{0}} \in$ $\mathcal{U}_{\mathbf{u}}^{\text {in }} \cap \bigcap_{j \in \mathbb{N}} \mathcal{U}_{\mathbf{u}_{j}}^{\text {in }}$ containing $\mathbf{x}_{0}$. Let $\mathbf{x}_{0} \in \Omega_{0}$ be such that $\mathbf{u}^{*}$ has a regular approximate differential at $\mathbf{x}_{0}$ with $\operatorname{det} \nabla \mathbf{u}\left(\mathbf{x}_{0}\right)>0$, and note by Proposition 2.6 that a.e. point in $\Omega$ satisfies this condition. In addition, $\mathbf{u}\left(\mathbf{x}_{0}\right)=\mathbf{u}^{*}\left(\mathbf{x}_{0}\right)$ and $\nabla \mathbf{u}\left(\mathbf{x}_{0}\right)=\nabla \mathbf{u}^{*}\left(\mathbf{x}_{0}\right)$ (recall Remark 2.4). By Lemmas 2.24 and 3.2 and Remark 2.19, there exist $r_{0}, r_{1}>0$ with $r_{0}<\frac{r_{1}}{2}$ such that, calling $U_{0}:=B\left(\mathbf{x}_{0}, r_{0}\right)$ and $U_{1}:=B\left(\mathbf{x}_{0}, r_{1}\right)$, we have that

$$
U_{0}, U_{1} \in \mathcal{U}_{\mathbf{u}} \cap \mathcal{U}_{\mathbf{u}^{*}} \cap \bigcap_{j \in \mathbb{N}}\left(\mathcal{U}_{\mathbf{u}_{j}} \cap \mathcal{U}_{\mathbf{u}_{j}^{*}}\right),
$$

$\mathbf{u}\left(\mathbf{x}_{0}\right) \notin \mathbf{u}\left(\partial U_{1}\right), \operatorname{deg}\left(\mathbf{u}, U_{1}, \mathbf{u}\left(\mathbf{x}_{0}\right)\right)=1, \mathbf{u}\left(\partial U_{0}\right)$ is included in the connected component of $\mathbb{R}^{n} \backslash \mathbf{u}\left(\partial U_{1}\right)$ containing $\mathbf{u}\left(\mathbf{x}_{0}\right)$,

$$
\left.\mathbf{u}\right|_{\partial U_{i}}=\left.\mathbf{u}^{*}\right|_{\partial U_{i}} \text { and }\left.\quad \mathbf{u}_{j}\right|_{\partial U_{i}}=\left.\mathbf{u}_{j}^{*}\right|_{\partial U_{i}} \quad \text { for each } j \in \mathbb{N} \quad \text { and } i \in\{1,2\}
$$


and, for a subsequence,

$$
\mathbf{u}_{j} \rightarrow \mathbf{u} \quad \text { uniformly in } \partial U_{i} \text { as } j \rightarrow \infty \text { for each } i \in\{1,2\} .
$$

Consequently, $\operatorname{deg}\left(\mathbf{u}, U_{1}, \cdot\right)=1$ in $\mathbf{u}\left(\partial U_{0}\right)$. By (6.2) and the fact that $\mathbf{u}\left(\partial U_{0}\right)$ and $\mathbf{u}\left(\partial U_{1}\right)$ are disjoint, $\operatorname{deg}\left(\mathbf{u}, U_{1}, \cdot\right)=1$ in $\mathbf{u}_{j}\left(\partial U_{0}\right)$ for large enough $j \in \mathbb{N}$, but now, thanks to Lemma 3.1 and (6.2) we have that $\operatorname{deg}\left(\mathbf{u}_{j}, U_{1}, \cdot\right)=1$ in $\mathbf{u}_{j}\left(\partial U_{0}\right)$ for large enough $j \in \mathbb{N}$. For such $j$, by Proposition $\left.4.5 c\right)$,

$$
\operatorname{deg}\left(\mathbf{u}, U_{1}, \cdot\right)=1 \text { in } \operatorname{im}_{\mathrm{T}}\left(\mathbf{u}, U_{0}\right) \text { and } \operatorname{deg}\left(\mathbf{u}_{j}, U_{1}, \cdot\right)=1 \text { in } \operatorname{im}_{\mathrm{T}}\left(\mathbf{u}_{j}, U_{0}\right)
$$

and, by Proposition $4.5 \mathrm{~b}$ ), $\mathbf{u}$ and $\mathbf{u}_{j}$ are one-to-one a.e. in $U_{0}$.

Thus, we have proved that for a.e. $\mathbf{x}_{0} \in \Omega$, for a subsequence there exists $U_{\mathbf{x}_{0}} \in \mathcal{U}_{\mathbf{u}}^{\text {in }} \cap \bigcap_{j \in \mathbb{N}} \mathcal{U}_{\mathbf{u}_{j}}^{\text {in }}$ containing $\mathbf{x}_{0}$. Thanks also to Lemma 2.24 , the family of $B \in \mathcal{U}_{\mathbf{u}}^{N} \cap \bigcap_{j \in \mathbb{N}} \mathcal{U}_{\mathbf{u}_{j}}^{N}$ such that $B \subset U_{\mathbf{x}_{0}}$ for some $\mathbf{x}_{0}$ as above and, in addition, $\mathbf{u}_{j} \rightarrow \mathbf{u}$ uniformly in $\partial B$, forms a fine covering of a.e. $\Omega$. Hence by Besicovitch's covering theorem, there exists a disjoint countable subfamily $\left\{B_{k}\right\}_{k \in \mathbb{N}}$ such that $\Omega=\bigcup_{k \in \mathbb{N}} B_{k}$ a.e. Employing a diagonal argument, we can extract a subsequence in $j$ such that (6.1) holds for each $k \in \mathbb{N}$.

Part c). By Proposition 5.3,

$$
\left(\left.\mathbf{u}\right|_{B}\right)^{-1} \in W^{1,1}\left(\operatorname{im}_{\mathrm{T}}(\mathbf{u}, B), \mathbb{R}^{n}\right) \quad \text { and } \quad D\left(\left.\mathbf{u}\right|_{B}\right)^{-1}=\left(D \mathbf{u} \circ\left(\left.\mathbf{u}\right|_{U}\right)^{-1}\right)^{-1} \text { a.e. }
$$

and, for all $j \in \mathbb{N}$,

$$
\left(\left.\mathbf{u}_{j}\right|_{B}\right)^{-1} \in W^{1,1}\left(\mathrm{im}_{\mathrm{T}}\left(\mathbf{u}_{j}, B\right), \mathbb{R}^{n}\right) \quad \text { and } \quad D\left(\left.\mathbf{u}_{j}\right|_{B}\right)^{-1}=\left(D \mathbf{u}_{j} \circ\left(\left.\mathbf{u}_{j}\right|_{B}\right)^{-1}\right)^{-1} \text { a.e. }
$$

Take a subsequence such that

$$
\mathbf{u}_{j} \rightarrow \mathbf{u} \text { a.e. in } \Omega \text { as } j \rightarrow \infty \text {. }
$$

We prove that, for each $\varphi \in C_{c}(V)$,

$$
\chi_{\left(\left.\mathbf{u}_{j}\right|_{B}\right)^{-1}\left(V \cap \operatorname{im}_{\mathrm{G}}\left(\mathbf{u}_{j}, B\right)\right)}(\mathbf{x}) \varphi\left(\mathbf{u}_{j}(\mathbf{x})\right) \rightarrow \chi_{\left(\left.\mathbf{u}\right|_{B}\right)^{-1}\left(V \cap \operatorname{im}_{\mathrm{G}}(\mathbf{u}, B)\right)}(\mathbf{x}) \varphi(\mathbf{u}(\mathbf{x})) \text { a.e. } \mathbf{x} \in \Omega \text {, as } j \rightarrow \infty .
$$

Indeed, given $\mathbf{x} \in \mathbb{R}^{n}$, as a consequence of Definition 5.2 , we have that

$$
\mathbf{x} \in\left(\left.\mathbf{u}\right|_{B}\right)^{-1}\left(V \cap \operatorname{im}_{\mathrm{G}}(\mathbf{u}, B)\right) \quad \text { if and only if } \mathbf{x} \in \Omega_{0} \cap B \text { and } \mathbf{u}(\mathbf{x}) \in V
$$

and, for large enough $j$,

$$
\mathbf{x} \in\left(\left.\mathbf{u}_{j}\right|_{B}\right)^{-1}\left(V \cap \operatorname{im}_{\mathrm{G}}\left(\mathbf{u}_{j}, B\right)\right) \quad \text { if and only if } \quad \mathbf{x} \in \Omega_{j} \cap B \text { and } \mathbf{u}_{j}(\mathbf{x}) \in V,
$$

where $\Omega_{0}$ is the set of Definition 2.11 corresponding to $\mathbf{u}$, and $\Omega_{j}$ is the corresponding one to $\mathbf{u}_{j}$. In order to show (6.4), take $\mathbf{x} \in \Omega_{0} \cap \bigcap_{j \in \mathbb{N}} \Omega_{j}$ satisfying $\mathbf{u}_{j}(\mathbf{x}) \rightarrow \mathbf{u}(\mathbf{x})$ as $j \rightarrow \infty$, and note that, thanks to (6.3), a.e. point in $\Omega$ fulfills these conditions. Then $\varphi\left(\mathbf{u}_{j}(\mathbf{x})\right) \rightarrow \varphi(\mathbf{u}(\mathbf{x}))$ as $j \rightarrow \infty$. If $\mathbf{u}(\mathbf{x}) \in V$, as $V$ is open, we have that $\mathbf{u}_{j}(\mathbf{x}) \in V$ for $j$ large enough, so, thanks to (6.5)-(6.6), convergence (6.4) holds for this $\mathbf{x}$, regardless of whether $\mathbf{x}$ belongs to $B$ or not. If, on the other hand, $\mathbf{u}(\mathbf{x}) \notin V$, then $\mathbf{u}(\mathbf{x}) \notin \operatorname{spt} \varphi$, and hence $\mathbf{u}_{j}(\mathbf{x}) \notin \operatorname{spt} \varphi$ for $j$ large enough, so convergence (6.4) holds for this $\mathbf{x}$ as well.

Now, let $M_{k}$ be a minor of order $k \in\{1, \ldots, n\}$. Using the formula (sometimes called Jacobi's equality) relating the minors of the inverse $\mathbf{F}^{-1}$ of a matrix $\mathbf{F} \in \mathbb{R}^{n \times n}$ in terms of the minors of $\mathbf{F}$ (see, e.g., [37, Sect. 1.4]), we can see that there exists a minor $N_{n-k}$ of order $n-k$ such that, up to a sign,

$$
M_{k}\left(\mathbf{F}^{-1}\right)=\frac{N_{n-k}(\mathbf{F})}{\operatorname{det} \mathbf{F}} \quad \text { for all invertible } \mathbf{F} \in \mathbb{R}^{n \times n} .
$$

For the sake of simplicity of notation, the possible sign change is incorporated in our definition of $N_{n-k}$, so that formula (6.7) holds true. Proposition 2.7, (5.1) and (6.7) show that, for $j \in \mathbb{N}$ large enough,

$$
\int_{V}\left|M_{k}\left(D\left(\left.\mathbf{u}_{j}\right|_{B}\right)^{-1}(\mathbf{y})\right)\right| \mathrm{d} \mathbf{y}=\int_{\left(\left.\mathbf{u}_{j}\right|_{B}\right)^{-1}\left(V \cap \operatorname{im}_{\mathrm{G}}(\mathbf{u}, B)\right)}\left|N_{n-k}\left(D \mathbf{u}_{j}(\mathbf{x})\right)\right| \mathrm{d} \mathbf{x} \leq c_{k, n} \int_{\Omega}\left|D \mathbf{u}_{j}(\mathbf{x})\right|^{n-k} \mathrm{~d} \mathbf{x},
$$


for some $c_{k, n}>0$ depending on $k$ and $n$. Therefore, $M_{k}\left(D\left(\left.\mathbf{u}_{j}\right|_{B}\right)^{-1}\right) \in L^{1}(V)$ and, similarly, $M_{k}\left(D\left(\left.\mathbf{u}\right|_{B}\right)^{-1}\right) \in$ $L^{1}(V)$. A classic result on the continuity of minors (see, e.g., [17, Th. 8.20]) shows that

$$
N_{n-k}\left(D \mathbf{u}_{j}\right) \rightarrow N_{n-k}(D \mathbf{u}) \quad \text { in } L^{1}(\Omega) \quad \text { as } j \rightarrow \infty .
$$

Now, by (5.1), Proposition 2.7 and (6.7) we have that for $j$ large enough and $\varphi \in C_{c}(V)$,

$$
\begin{aligned}
\int_{V} \varphi(\mathbf{y}) M_{k}\left(D\left(\left.\mathbf{u}_{j}\right|_{B}\right)^{-1}(\mathbf{y})\right) \mathrm{d} \mathbf{y} & =\int_{V \cap \mathrm{im}_{\mathrm{G}}\left(\mathbf{u}_{j}, B\right)} \varphi(\mathbf{y}) M_{k}\left(D\left(\left.\mathbf{u}_{j}\right|_{B}\right)^{-1}(\mathbf{y})\right) \mathrm{d} \mathbf{y} \\
& =\int_{\left(\left.\mathbf{u}_{j}\right|_{B}\right)^{-1}\left(V \cap \operatorname{im}_{\mathrm{G}}\left(\mathbf{u}_{j}, B\right)\right)} \varphi\left(\mathbf{u}_{j}(\mathbf{x})\right) N_{n-k}\left(D \mathbf{u}_{j}(\mathbf{x})\right) \mathrm{d} \mathbf{x}
\end{aligned}
$$

and, similarly,

$$
\int_{V} \varphi(\mathbf{y}) M_{k}\left(D\left(\left.\mathbf{u}\right|_{B}\right)^{-1}(\mathbf{y})\right) \mathrm{d} \mathbf{y}=\int_{\left(\left.\mathbf{u}\right|_{B}\right)^{-1}\left(V \cap \operatorname{im}_{\mathrm{G}}(\mathbf{u}, B)\right)} \varphi(\mathbf{u}(\mathbf{x})) N_{n-k}(D \mathbf{u}(\mathbf{x})) \mathrm{d} \mathbf{x} .
$$

By (6.4), (6.9) and the convergence result [33, Prop. 2.61], we find that

$$
\lim _{j \rightarrow \infty} \int_{\left(\left.\mathbf{u}_{j}\right|_{B}\right)^{-1}\left(V \cap \operatorname{im}_{\mathrm{G}}\left(\mathbf{u}_{j}, B\right)\right)} \varphi\left(\mathbf{u}_{j}(\mathbf{x})\right) N_{n-k}\left(D \mathbf{u}_{j}(\mathbf{x})\right) \mathrm{d} \mathbf{x}=\int_{\left(\left.\mathbf{u}\right|_{B}\right)^{-1}\left(V \cap \operatorname{im}_{\mathrm{G}}(\mathbf{u}, B)\right)} \varphi(\mathbf{u}(\mathbf{x})) N_{n-k}(D \mathbf{u}(\mathbf{x})) \mathrm{d} \mathbf{x},
$$

so thanks to (6.10)-(6.11) we obtain that

$$
\lim _{j \rightarrow \infty} \int_{V} \varphi(\mathbf{y}) M_{k}\left(D\left(\left.\mathbf{u}_{j}\right|_{B}\right)^{-1}(\mathbf{y})\right) \mathrm{d} \mathbf{y}=\int_{V} \varphi(\mathbf{y}) M_{k}\left(D\left(\left.\mathbf{u}\right|_{B}\right)^{-1}(\mathbf{y})\right) \mathrm{d} \mathbf{y}
$$

which proves c2). In particular, $D\left(\left.\mathbf{u}_{j}\right|_{B}\right)^{-1} \stackrel{*}{\rightarrow} D\left(\left.\mathbf{u}\right|_{B}\right)^{-1}$ in $\mathcal{M}\left(V, \mathbb{R}^{n \times n}\right)$ as $j \rightarrow \infty$. As $\left\{\left(\left.\mathbf{u}_{j}\right|_{B}\right)^{-1}\right\}_{j \in \mathbb{N}}$ is bounded in $L^{\infty}\left(V, \mathbb{R}^{n}\right)$, we infer, under the additional assumption that $V$ is connected, that there exists $\mathbf{c} \in \mathbb{R}^{n}$ such that, for a subsequence,

$$
\left(\left.\mathbf{u}_{j}\right|_{B}\right)^{-1} \rightarrow\left(\left.\mathbf{u}\right|_{B}\right)^{-1}+\mathbf{c} \text { a.e. in } V \text { as } j \rightarrow \infty .
$$

If $V$ is not connected, we argue in each connected component, since in any case we shall show that $\mathbf{c}=$ 0. Assume, by contradiction, that $\mathbf{c} \neq \mathbf{0}$ and take a ball $U \in \mathcal{U}_{\mathbf{u}} \cap \bigcap_{j \in \mathbb{N}} \mathcal{U}_{\mathbf{u}_{j}}$ contained in $B$ of radius smaller than $|\mathbf{c}| / 2$ for which $\mathbf{u}_{j} \rightarrow \mathbf{u}$ uniformly in $\partial U$ as $j \rightarrow \infty$, which is possible thanks to Lemma 2.24. Let $W \subset \subset \operatorname{im}_{\mathrm{T}}(\mathbf{u}, U)$ be open and non-empty. Then, by Lemma 3.6 there exists $j_{W} \in \mathbb{N}$ such that $W \subset \operatorname{im}_{\mathrm{T}}\left(\mathbf{u}_{j}, U\right)$ for all $j \geq j_{W}$. Thanks to (5.1) and (6.12), for a.e. $\mathbf{y} \in W$ and large enough $j \in \mathbb{N}$ there exist $\mathbf{x}_{j} \in U \cap \Omega_{j}$ and $\mathbf{a} \in U \cap \Omega_{0}$ such that $\mathbf{y}=\mathbf{u}_{j}\left(\mathbf{x}_{j}\right)=\mathbf{u}(\mathbf{a})$ and $\left(\left.\mathbf{u}_{j}\right|_{B}\right)^{-1}(\mathbf{y}) \rightarrow\left(\left.\mathbf{u}\right|_{B}\right)^{-1}(\mathbf{y})+\mathbf{c}$, that is to say, $\mathbf{x}_{j} \rightarrow \mathbf{a}+\mathbf{c}$ as $j \rightarrow \infty$. Therefore, $\mathbf{a}+\mathbf{c} \in \bar{U}$, which is impossible because $\mathbf{a} \in U$ and the radius of $U$ is smaller than $|\mathbf{c}| / 2$. Thus, $\mathbf{c}=\mathbf{0}$ and convergence $c 1$ ) is proved.

If, in addition, the sequence $\left\{\operatorname{det} D\left(\left.\mathbf{u}_{j}\right|_{B}\right)^{-1}\right\}_{j \in \mathbb{N}}$ is equiintegrable in $V$, then so is $\left\{M_{k}\left(D\left(\left.\mathbf{u}_{j}\right|_{B}\right)^{-1}\right)\right\}_{j \in \mathbb{N}}$ for any minor $M_{k}$ of order $k \in\{1, \ldots, n-1\}$. Indeed, call $q:=\frac{p}{n-k}$ and notice that, thanks to Hölder's inequality, we have that for any measurable set $A \subset V$,

$$
\int_{A}\left|M_{k}\left(D\left(\left.\mathbf{u}_{j}\right|_{B}\right)^{-1}(\mathbf{y})\right)\right| \mathrm{d} \mathbf{y} \leq\left(\int_{A} \frac{\left|M_{k}\left(D\left(\left.\mathbf{u}_{j}\right|_{B}\right)^{-1}(\mathbf{y})\right)\right|^{q}}{\left(\operatorname{det} D\left(\left.\mathbf{u}_{j}\right|_{B}\right)^{-1}(\mathbf{y})\right)^{\frac{q}{q^{\prime}}}} \mathrm{d} \mathbf{y}\right)^{\frac{1}{q}}\left(\int_{A} \operatorname{det} D\left(\left.\mathbf{u}_{j}\right|_{B}\right)^{-1}(\mathbf{y}) \mathrm{d} \mathbf{y}\right)^{\frac{1}{q^{\prime}}}
$$

and, arguing as in (6.8),

$$
\int_{A} \frac{\left|M_{k}\left(D\left(\left.\mathbf{u}_{j}\right|_{B}\right)^{-1}(\mathbf{y})\right)\right|^{q}}{\left(\operatorname{det} D\left(\left.\mathbf{u}_{j}\right|_{B}\right)^{-1}(\mathbf{y})\right)^{q} q^{q}} \mathrm{~d} \mathbf{y}=\int_{\left(\left.\mathbf{u}_{j}\right|_{B}\right)^{-1}(A)}\left|N_{n-k}\left(D \mathbf{u}_{j}(\mathbf{x})\right)\right|^{q} \mathrm{~d} \mathbf{x} \leq c_{k, n}^{q} \int_{\Omega}\left|D \mathbf{u}_{j}(\mathbf{x})\right|^{p} \mathrm{~d} \mathbf{x} .
$$


Therefore, $M_{k}\left(D\left(\left.\mathbf{u}_{j}\right|_{B}\right)^{-1}\right) \rightarrow M_{k}\left(D\left(\left.\mathbf{u}\right|_{B}\right)^{-1}\right)$ in $L^{1}(V)$ as $j \rightarrow \infty$ for any minor $M_{k}$ of order $k \in\{1, \ldots, n\}$, so the convergence in $c 2)$ holds in the weak topology of $L^{1}(V)$, and the convergence in $\left.c 1\right)$ holds in the weak topology of $W^{1,1}\left(V, \mathbb{R}^{n}\right)$.

Part d). Thanks to Lemma 2.24, for each $\mathbf{x}_{0} \in \Omega$ there exists a ball $B \in \mathcal{U}_{\mathbf{u}} \cap \bigcap_{j \in \mathbb{N}} \mathcal{U}_{\mathbf{u}_{j}}$ centred at $\mathbf{x}_{0}$ such that, for a subsequence, $\mathbf{u}_{j} \rightarrow \mathbf{u}$ uniformly in $\partial B$ as $j \rightarrow \infty$. By the Lindelöf property, there exists a countable family $\left\{B_{k}\right\}_{k \in \mathbb{N}}$ of such balls such that $\Omega=\bigcup_{k \in \mathbb{N}} B_{k}$. Applying a standard diagonal argument, we can find a subsequence such that for all $k \in \mathbb{N}$,

$$
\mathbf{u}_{j} \rightarrow \mathbf{u} \quad \text { uniformly in } \partial B_{k} \text { as } j \rightarrow \infty \text {. }
$$

By Lemma 3.6 we have

$$
\chi_{\operatorname{im}_{\mathrm{T}}\left(\mathbf{u}_{j}, B_{k}\right)} \rightarrow \chi_{\mathrm{im}_{\mathrm{T}}\left(\mathbf{u}, B_{k}\right)} \quad \text { in } L^{1}\left(\mathbb{R}^{n}\right) \text { as } j \rightarrow \infty .
$$

Using Lemma $5.18 c$ ) we find that

$$
\mathcal{L}^{n}\left(\operatorname{im}_{\mathrm{T}}\left(\mathbf{u}_{j}, \Omega\right) \triangle \operatorname{im}_{\mathrm{T}}(\mathbf{u}, \Omega)\right)=\mathcal{L}^{n}\left(\operatorname{im}_{\mathrm{G}}\left(\mathbf{u}_{j}, \Omega\right) \triangle \operatorname{im}_{\mathrm{G}}(\mathbf{u}, \Omega)\right) \leq \sum_{k \in \mathbb{N}} \mathcal{L}^{n}\left(\operatorname{im}_{\mathrm{G}}\left(\mathbf{u}_{j}, B_{k}\right) \triangle \operatorname{im}_{\mathrm{G}}\left(\mathbf{u}, B_{k}\right)\right),
$$

so applying (6.13), (5.1) and a standard diagonal argument, we obtain the existence of a subsequence for which the convergence of $d$ ) holds.

\section{Lower semicontinuity}

In this section we exploit Theorem 6.3 and, hence, the possibility of working in the deformed configuration, to prove some lower semicontinuity results. As seen in Lemma $5.18, \operatorname{im}_{\mathrm{T}}(\mathbf{u}, \Omega)$ is open, does not depend on the representative of $\mathbf{u}$ and $\operatorname{im}_{\mathrm{T}}(\mathbf{u}, \Omega)=\operatorname{im}_{\mathrm{G}}(\mathbf{u}, \Omega)$ a.e. These features make $\operatorname{im}_{\mathrm{T}}(\mathbf{u}, \Omega)$ a natural definition of deformed configuration.

This section consists of three subsections. In Subsection 7.1 we analyze integral functionals involving variations of the domain under the assumption of quasiconvexity. In Subsection 7.2 we prove that Divquasiconvexity is a sufficient condition for lower semicontinuity under the incompressibility constraint. In Subsection 7.3 we study polyconvex functionals that involve a composition of maps. The results of Subsection 7.1 and 7.3 will be applied in Section 8 to actual physical models.

\subsection{Quasiconvex functionals in the deformed configuration}

We analyze integral functionals defined in the deformed configuration of the type

$$
I(\mathbf{w}, \mathbf{u}):=\int_{\operatorname{im}_{\mathrm{T}}(\mathbf{u}, \Omega)} W(D \mathbf{w}(\mathbf{y})) \mathrm{d} \mathbf{y}
$$

where $d \in \mathbb{N}, W: \mathbb{R}^{d \times n} \rightarrow \mathbb{R}$ is a given density, $\mathbf{u} \in \mathcal{A}_{p}$, and $\mathbf{w}: \operatorname{im}_{\mathrm{T}}(\mathbf{u}, \Omega) \rightarrow \mathbb{R}^{d}$ is an additional map. This kind of functionals were initially studied in Fonseca \& Parry $[35,36]$ to describe the equilibria of crystals with defects within a variational framework.

In general, the direct method of the calculus of variations may fail to provide existence of minima of (7.1). The essence of that difficulty is the lack of compactness. Indeed, while bounds on $I$ imply bounds on $D \mathbf{w}$ under certain coercivity conditions on $W$, in no way do they imply bounds on $D \mathbf{u}$ (see the discussion in $[18,32])$. We will see in Section 8 that the necessary compactness will be achieved by adding a suitable term to the energy, which, as a matter of fact, will be analyzed in Subsection 7.3.

In this subsection we analyze the semicontinuity of (7.1). The main difficulty consists in the varying domain $\operatorname{im}_{\mathrm{T}}\left(\mathbf{u}_{j}, \Omega\right)$ for a minimizing sequence $\left\{\left(\mathbf{w}_{j}, \mathbf{u}_{j}\right)\right\}_{j \in \mathbb{N}}$ and, in particular, in the compactness of $\left\{\mathbf{w}_{j}\right\}_{j \in \mathbb{N}}$

with $\left\{\left\|\mathbf{w}_{j}\right\|_{W^{1, r}\left(\operatorname{im}_{\mathrm{T}}\left(\mathbf{u}_{j}, \Omega\right), \mathbb{R}^{d}\right)}\right\}_{j \in \mathbb{N}}$ bounded. Assuming the convergence $\mathbf{u}_{j} \rightarrow \mathbf{u}$ in $W^{1, p}\left(\Omega, \mathbb{R}^{n}\right)$, thanks to Theorem 6.3 we will have compactness of $\left\{\left.\mathbf{w}_{j}\right|_{V}\right\}_{j \in \mathbb{N}}$ for each subdomain $V \subset \subset \operatorname{im}_{\mathrm{T}}(\mathbf{u}, U)$ with $U \in \mathcal{U}_{\mathbf{u}}^{N}$. 
The results of this section require the quasiconvexity of $W$. Recall (see, e.g., [17, Def. 5.1]) that a Borel and locally bounded function $W: \mathbb{R}^{d \times n} \rightarrow \mathbb{R}$ is quasiconvex if

$$
W(\mathbf{F}) \leq \int_{(0,1)^{n}} W(\mathbf{F}+D \varphi(\mathbf{x})) \mathrm{d} \mathbf{x}
$$

for every $\mathbf{F} \in \mathbb{R}^{d \times n}$ and every $\varphi \in W_{0}^{1, \infty}\left((0,1)^{n}, \mathbb{R}^{d}\right)$.

The proof of the following result is inspired in that of Barchiesi \& DeSimone [11], but we lower the exponent of integrability from $p \geq n$ to $p>n-1$.

Proposition 7.1. Let $r>1, p>n-1, d \in \mathbb{N}$ and $K$ a closed subset of $\mathbb{R}^{d}$. For each $j \in \mathbb{N}$, let $\mathbf{u}, \mathbf{u}_{j} \in \mathcal{A}_{p}$ and $\mathbf{w}_{j} \in W^{1, r}\left(\operatorname{im}_{\mathrm{T}}\left(\mathbf{u}_{j}, \Omega\right), K\right)$ be such that

$$
\mathbf{u}_{j} \rightarrow \mathbf{u} \text { in } W^{1, p}\left(\Omega, \mathbb{R}^{n}\right) \quad \text { as } j \rightarrow \infty \quad \text { and } \quad \sup _{j \in \mathbb{N}}\left[\left\|\mathbf{w}_{j}\right\|_{L^{1}\left(\mathrm{im}_{\mathrm{T}}\left(\mathbf{u}_{j}, \Omega\right), \mathbb{R}^{d}\right)}+\left\|D \mathbf{w}_{j}\right\|_{L^{r}\left(\mathrm{im}_{\mathrm{T}}\left(\mathbf{u}_{j}, \Omega\right), \mathbb{R}^{d \times n}\right)}\right]<\infty .
$$

Let $W: \mathbb{R}^{d \times n} \rightarrow[0, \infty)$ be a quasiconvex function for which there is a $c>0$ with

$$
W(\mathbf{F}) \leq c\left(1+|\mathbf{F}|^{r}\right), \quad \mathbf{F} \in \mathbb{R}^{d \times n} .
$$

Then there exists $\mathbf{w} \in W^{1, r}\left(\operatorname{im}_{\mathrm{T}}(\mathbf{u}, \Omega), K\right)$ such that

$$
\int_{\operatorname{im}_{\mathrm{T}}(\mathbf{u}, \Omega)} W(D \mathbf{w}(\mathbf{y})) \mathrm{d} \mathbf{y} \leq \liminf _{j \rightarrow \infty} \int_{\operatorname{im}_{\mathrm{T}}\left(\mathbf{u}_{j}, \Omega\right)} W\left(D \mathbf{w}_{j}(\mathbf{y})\right) \mathrm{d} \mathbf{y}
$$

and, for a subsequence,

$$
\begin{aligned}
& \chi_{\mathrm{im}_{\mathrm{T}}\left(\mathbf{u}_{j}, \Omega\right)} \mathbf{w}_{j} \rightarrow \chi_{\mathrm{im}_{\mathrm{T}}(\mathbf{u}, \Omega)} \mathbf{w} \text { a.e., } \\
& \chi_{\operatorname{im}_{\mathrm{T}}\left(\mathbf{u}_{j}, \Omega\right)} D \mathbf{w}_{j} \rightarrow \chi_{\mathrm{im}_{\mathrm{T}}(\mathbf{u}, \Omega)} D \mathbf{w} \text { in } L^{r}\left(\mathbb{R}^{n}, \mathbb{R}^{d \times n}\right) \quad \text { as } j \rightarrow \infty .
\end{aligned}
$$

If, in addition, $\operatorname{det} D \mathbf{u} \in L^{1}(\Omega)$ and $K$ is compact then

$$
\chi_{\mathrm{im}_{\mathrm{T}}\left(\mathbf{u}_{j}, \Omega\right)} \mathbf{w}_{j} \rightarrow \chi_{\mathrm{im}_{\mathrm{T}}(\mathbf{u}, \Omega)} \mathbf{w} \text { in } L^{r}\left(\mathbb{R}^{n}, \mathbb{R}^{d}\right) \quad \text { as } j \rightarrow \infty .
$$

In (7.4)-(7.5), $\chi_{\mathrm{im}_{\mathrm{T}}\left(\mathbf{u}_{j}, \Omega\right)} \mathbf{w}_{j}$ and $\chi_{\mathrm{im}_{\mathrm{T}}(\mathbf{u}, \Omega)} \mathbf{w}$ denote, respectively, the extensions to $\mathbb{R}^{n}$ of $\mathbf{w}_{j}$ and $\mathbf{w}$ by zero; analogous meaning for $\chi_{\mathrm{im}_{\mathrm{T}}\left(\mathbf{u}_{j}, \Omega\right)} D \mathbf{w}_{j}$ and $\chi_{\mathrm{im}_{\mathrm{T}}(\mathbf{u}, \Omega)} D \mathbf{w}$. In the proof of this proposition we will make use of the biting convergence (see [13, 10] or else, e.g., [38, Def. 1.2.7.1], [2, Lemma 5.32]); the precise definition is not important in the proof but only the fact that one can find a subsequence and a limit in $L^{1}$ from a bounded sequence in $L^{1}$.

Proof. For a subsequence, we can assume that the liminf of the right-hand side of (7.3) is actually a limit. For each $j \in \mathbb{N}$, let $\overline{\mathbf{w}}_{j}$ and $\mathbf{W}_{j}$ be the extensions of $\mathbf{w}_{j}$ and $D \mathbf{w}_{j}$, respectively, by zero to $\mathbb{R}^{n}$. Since $\left\{\overline{\mathbf{w}}_{j}\right\}_{j \in \mathbb{N}}$ is bounded in $L^{1}\left(\mathbb{R}^{n}, \mathbb{R}^{d}\right)$ and $\left\{\mathbf{W}_{j}\right\}_{j \in \mathbb{N}}$ is bounded in $L^{r}\left(\mathbb{R}^{n}, \mathbb{R}^{d \times n}\right)$, there exist $\overline{\mathbf{w}} \in L^{1}\left(\mathbb{R}^{n}, \mathbb{R}^{d}\right)$ and $\mathbf{W} \in L^{r}\left(\mathbb{R}^{n}, \mathbb{R}^{d \times n}\right)$ such that, for a subsequence,

$$
\overline{\mathbf{w}}_{j} \stackrel{b}{\rightarrow} \overline{\mathbf{w}} \quad \text { in } L^{1}\left(\mathbb{R}^{n}, \mathbb{R}^{d}\right) \quad \text { and } \quad \mathbf{W}_{j} \rightarrow \mathbf{W} \quad \text { in } L^{r}\left(\mathbb{R}^{n}, \mathbb{R}^{d \times n}\right) \quad \text { as } j \rightarrow \infty .
$$

We have denoted by $\stackrel{b}{\rightarrow}$ convegence in the biting sense.

Let $\mathbf{y} \in \operatorname{im}_{\mathrm{T}}(\mathbf{u}, \Omega)$. Then there exist $U \in \mathcal{U}_{\mathbf{u}}^{N}$ and a smooth open set $V \subset \subset \operatorname{im}_{\mathrm{T}}(\mathbf{u}, U)$ such that $\mathbf{y} \in V$. By Theorem $6.3 a$ ), for a subsequence, $V \subset \operatorname{im}_{\mathrm{T}}\left(\mathbf{u}_{j}, \Omega\right)$ for all $j \in \mathbb{N}$, so $\left.\overline{\mathbf{w}}_{j}\right|_{V}=\left.\mathbf{w}_{j}\right|_{V}$. Since $\left\{\mathbf{w}_{j}\right\}_{j \in \mathbb{N}}$ is bounded in $W^{1,1}\left(V, \mathbb{R}^{d}\right)$ and $\left\{D \mathbf{w}_{j}\right\}_{j \in \mathbb{N}}$ is bounded in $L^{r}\left(V, \mathbb{R}^{d \times n}\right)$, a bootstrap argument based on the Sobolev embedding (or else a version of the Poincaré inequality; see, e.g., [58, Sect. 1.1.11], if necesary) shows that $\left\{\mathbf{w}_{j}\right\}_{j \in \mathbb{N}}$ is bounded in $W^{1, r}\left(V, \mathbb{R}^{d}\right)$. Thus, by (7.6) we have

$$
\mathbf{w}_{j} \rightarrow \overline{\mathbf{w}} \text { in } W^{1, r}\left(V, \mathbb{R}^{d}\right) \text { and } \quad \mathbf{w}_{j} \rightarrow \overline{\mathbf{w}} \text { a.e. in } V, \quad \text { as } j \rightarrow \infty,
$$


again for a subsequence. Thus, $\overline{\mathbf{w}} \in W^{1, r}\left(V, \mathbb{R}^{n}\right)$ and $\mathbf{W}=D \overline{\mathbf{w}}$ a.e. in $V$. In fact, as $K$ is closed, $\overline{\mathbf{w}} \in W^{1, r}(V, K)$. Therefore, $\overline{\mathbf{w}} \in W_{\text {loc }}^{1, r}\left(\operatorname{im}_{\mathrm{T}}(\mathbf{u}, \Omega), \mathbb{R}^{n}\right)$. Moreover, by the Lindelöf property, $\operatorname{im}_{\mathrm{T}}(\mathbf{u}, \Omega)$ can be expressed as a countable union of sets $V$ as before, hence $\overline{\mathbf{w}}_{j} \rightarrow \overline{\mathbf{w}}$ a.e. in $\operatorname{im}_{\mathrm{T}}(\mathbf{u}, \Omega), \overline{\mathbf{w}} \in W_{\mathrm{loc}}^{1, r}\left(\operatorname{im}_{\mathrm{T}}(\mathbf{u}, \Omega), K\right)$ and $D \overline{\mathbf{w}}=\mathbf{W}$ a.e. in $\operatorname{im}_{\mathrm{T}}(\mathbf{u}, \Omega)$, so that actually $\overline{\mathbf{w}} \in W^{1, r}\left(\operatorname{im}_{\mathrm{T}}(\mathbf{u}, \Omega), K\right)$.

Define $\mathbf{w}:=\left.\overline{\mathbf{w}}\right|_{\mathrm{im}_{\mathrm{T}}(\mathbf{u}, \Omega)}$. Due to $(7.7)$ and Theorem $6.3 d$ ) we immediately have the first convergence of (7.4). Having in mind (7.6) and (7.7), the second convergence will be completed as soon as we show that $\mathbf{W}=\mathbf{0}$ a.e. in $\mathbb{R}^{n} \backslash \operatorname{im}_{\mathrm{T}}(\mathbf{u}, \Omega)$. In order to achieve this, we notice that given any bounded measurable set $C \subset \mathbb{R}^{n} \backslash \operatorname{im}_{\mathrm{T}}(\mathbf{u}, \Omega)$,

$$
\left|\int_{C} \mathbf{W} \mathrm{d} \mathbf{y}\right|=\lim _{j \rightarrow \infty}\left|\int_{C} \mathbf{W}_{j} \mathrm{~d} \mathbf{y}\right| \leq \lim _{j \rightarrow \infty} \int_{\operatorname{im}_{\mathrm{T}}\left(\mathbf{u}_{j}, \Omega\right) \backslash \operatorname{im}_{\mathrm{T}}(\mathbf{u}, \Omega)}\left|\mathbf{W}_{j}\right| \mathrm{d} \mathbf{y}=0,
$$

where for the last equality we have used Theorem $6.3 d$ ) and the equiintegrability of $\left\{\mathbf{W}_{j}\right\}_{j \in \mathbb{N}}$. Therefore, $\mathbf{W}=\chi_{\mathrm{im}_{\mathrm{T}}(\mathbf{u}, \Omega)} D \mathbf{w}$ and the second convergence of (7.4) holds.

Let now $\left\{V_{k}\right\}_{k \in \mathbb{N}}$ be the family of Lemma $5.18 d$ ). Then,

$$
\int_{\operatorname{im}_{\mathrm{T}}(\mathbf{u}, \Omega)} W(D \mathbf{w}) \mathrm{d} \mathbf{y}=\sum_{k \in \mathbb{N}} \int_{V_{k}} W(D \mathbf{w}) \mathrm{d} \mathbf{y}
$$

and, for each $j \in \mathbb{N}$,

$$
\int_{\operatorname{im}_{\mathrm{T}}\left(\mathbf{u}_{j}, \Omega\right) \cap \mathrm{im}_{\mathrm{T}}(\mathbf{u}, \Omega)} W\left(D \mathbf{w}_{j}\right) \mathrm{d} \mathbf{y}=\sum_{k \in \mathbb{N}} \int_{\operatorname{im}_{\mathrm{T}}\left(\mathbf{u}_{j}, \Omega\right) \cap V_{k}} W\left(D \mathbf{w}_{j}\right) \mathrm{d} \mathbf{y} .
$$

For each $k \in \mathbb{N}$, let $B_{k} \in \mathcal{U}_{\mathbf{u}}^{N}$ be such that $V_{k} \subset \subset \operatorname{im}_{\mathrm{T}}\left(\mathbf{u}, B_{k}\right)$. Since, by Theorem $\left.6.3 a\right), V_{k} \subset \operatorname{im}_{\mathrm{T}}\left(\mathbf{u}_{j}, \Omega\right)$ for a subsequence, thanks to the bound in (7.2) and the convergences of (7.6), we obtain, arguing as in (7.7), that $\mathbf{w}_{j} \rightarrow \mathbf{w}$ in $W^{1, r}\left(V_{k}, \mathbb{R}^{d}\right)$ as $j \rightarrow \infty$. By the lower semicontinuity theorem [17, Th. 8.4] for quasiconvex functions,

$$
\int_{V_{k}} W(D \mathbf{w}) \mathrm{d} \mathbf{y} \leq \liminf _{j \rightarrow \infty} \int_{V_{k}} W\left(D \mathbf{w}_{j}\right) \mathrm{d} \mathbf{y}=\liminf _{j \rightarrow \infty} \int_{\mathrm{im}_{\mathrm{T}}\left(\mathbf{u}_{j}, \Omega\right) \cap V_{k}} W\left(D \mathbf{w}_{j}\right) \mathrm{d} \mathbf{y} .
$$

Putting together equations (7.8)-(7.9) we obtain

$$
\begin{aligned}
\int_{\operatorname{im}_{\mathrm{T}}(\mathbf{u}, \Omega)} W(D \mathbf{w}) \mathrm{d} \mathbf{y} & \leq \sum_{k \in \mathbb{N}} \liminf _{j \rightarrow \infty} \int_{\operatorname{im}_{\mathrm{T}}\left(\mathbf{u}_{j}, \Omega\right) \cap V_{k}} W\left(D \mathbf{w}_{j}\right) \mathrm{d} \mathbf{y} \\
& \leq \liminf _{j \rightarrow \infty} \int_{\operatorname{im}_{\mathrm{T}}\left(\mathbf{u}_{j}, \Omega\right) \cap \operatorname{im}_{\mathrm{T}}(\mathbf{u}, \Omega)} W\left(D \mathbf{w}_{j}\right) \mathrm{d} \mathbf{y} \\
& \leq \liminf _{j \rightarrow \infty} \int_{\operatorname{im}_{\mathrm{T}}\left(\mathbf{u}_{j}, \Omega\right)} W\left(D \mathbf{w}_{j}\right) \mathrm{d} \mathbf{y}
\end{aligned}
$$

which concludes the proof of $(7.3)$.

We finally show convergence (7.5) under the assumption that $K$ is compact and det $D \mathbf{u} \in L^{1}(\Omega)$. Let $M>0$ be such that $|\mathbf{z}| \leq M$ for all $\mathbf{z} \in K$. Consider the family $\left\{B_{k}\right\}_{k \in \mathbb{N}}$ of Theorem $\left.6.3 b\right)$ and the subsequence therein. Fix $\varepsilon>0$, and let $k_{\varepsilon} \in \mathbb{N}$ be such that

$$
\mathcal{L}^{n}\left(\bigcup_{k>k_{\varepsilon}} \operatorname{im}_{\mathrm{T}}\left(\mathbf{u}, B_{k}\right)\right) \leq \frac{\varepsilon}{(2 M)^{r}}
$$

which is possible since, by (5.1), Proposition 2.7 and the assumption $\operatorname{det} D \mathbf{u} \in L^{1}(\Omega)$,

$$
\sum_{k \in \mathbb{N}} \mathcal{L}^{n}\left(\operatorname{im}_{\mathrm{T}}\left(\mathbf{u}, B_{k}\right)\right)=\sum_{k \in \mathbb{N}} \mathcal{L}^{n}\left(\operatorname{im}_{\mathrm{G}}\left(\mathbf{u}, B_{k}\right)\right) \leq \sum_{k \in \mathbb{N}} \int_{B_{k}} \operatorname{det} D \mathbf{u} \mathrm{d} \mathbf{x}<\infty
$$


For each $k \in \mathbb{N}$, let $W_{k} \subset \subset \operatorname{im}_{\mathrm{T}}\left(\mathbf{u}, B_{k}\right)$ be a smooth open set such that

$$
\mathcal{L}^{n}\left(\operatorname{im}_{\mathrm{T}}\left(\mathbf{u}, B_{k}\right) \backslash W_{k}\right) \leq 2^{-k} \frac{\varepsilon}{(2 M)^{r}} .
$$

By Lemma 3.6, $W_{k} \subset \operatorname{im}_{\mathrm{T}}\left(\mathbf{u}_{j}, B_{k}\right)$ for all $j \in \mathbb{N}$, provided that a subsequence has been selected. Arguing as in (7.7), we obtain that $\left.\overline{\mathbf{w}}_{j}\right|_{W_{k}}=\left.\mathbf{w}_{j}\right|_{W_{k}}$, the sequence $\left\{\overline{\mathbf{w}}_{j}\right\}_{j \in \mathbb{N}}$ is bounded in $W^{1, r}\left(W_{k}, \mathbb{R}^{d}\right)$ and

$$
\overline{\mathbf{w}}_{j} \rightarrow \overline{\mathbf{w}} \quad \text { in } L^{r}\left(W_{k}, \mathbb{R}^{d}\right) \quad \text { as } j \rightarrow \infty,
$$

again for a subsequence, which, by a diagonal argument, can be assumed to be valid for all $k \in \mathbb{N}$. We now have

$$
\int_{\mathbb{R}^{n}}\left|\overline{\mathbf{w}}_{j}-\overline{\mathbf{w}}\right|^{r} \mathrm{~d} \mathbf{y} \leq \int_{\operatorname{im}_{\mathrm{T}}(\mathbf{u}, \Omega)}\left|\overline{\mathbf{w}}_{j}-\overline{\mathbf{w}}\right|^{r} \mathrm{~d} \mathbf{y}+M^{r} \mathcal{L}^{n}\left(\operatorname{im}_{\mathrm{T}}\left(\mathbf{u}_{j}, \Omega\right) \backslash \operatorname{im}_{\mathrm{T}}(\mathbf{u}, \Omega)\right),
$$

and, using (7.10) and (7.11),

$$
\int_{\operatorname{im}_{\mathrm{T}}(\mathbf{u}, \Omega)}\left|\overline{\mathbf{w}}_{j}-\overline{\mathbf{w}}\right|^{r} \mathrm{~d} \mathbf{y} \leq \sum_{k=1}^{k_{\varepsilon}} \int_{\operatorname{im}_{\mathrm{T}}\left(\mathbf{u}, B_{k}\right)}\left|\overline{\mathbf{w}}_{j}-\overline{\mathbf{w}}\right|^{r} \mathrm{~d} \mathbf{y}+\varepsilon \leq \sum_{k=1}^{k_{\varepsilon}} \int_{W_{k}}\left|\overline{\mathbf{w}}_{j}-\overline{\mathbf{w}}\right|^{r} \mathrm{~d} \mathbf{y}+2 \varepsilon .
$$

Thanks to convergence (7.12) for each $k \in \mathbb{N}$, Theorem $6.3 d$ ) and equations (7.13) and (7.14) conclude the proof of (7.5).

We now explain an alternative form of (7.1), as well as the corresponding lower semicontinuity result; for this we follow $[35,36,18,32]$. When we introduce the function $\mathbf{v}:=\mathbf{w} \circ \mathbf{u}$, the functional $I$ of $(7.1)$ can be formally rewritten as

$$
\int_{\Omega} W\left(D \mathbf{v}(\mathbf{x})(D \mathbf{u}(\mathbf{x}))^{-1}\right) \mathrm{d} \mathbf{x}
$$

under the additional incompressibility constraint $\operatorname{det} D \mathbf{u}=1$ a.e. and the assumption that $\mathbf{u}$ is one-to-one a.e. Since $(D \mathbf{u})^{-1}=\operatorname{adj} D \mathbf{u} \in L^{\frac{p}{n-1}}\left(\Omega, \mathbb{R}^{n \times n}\right)$, in order to have the integrability of $D \mathbf{v}(D \mathbf{u})^{-1}$, we need to assume that $\mathbf{v} \in W^{1, q}\left(\Omega, \mathbb{R}^{d}\right)$ with $q \geq\left(\frac{p}{n-1}\right)^{\prime}$. The following version of the chain rule for $\mathbf{w}$ holds.

Lemma 7.2. Let $p>n-1, d \in \mathbb{N}$ and $q:=\left(\frac{p}{n-1}\right)^{\prime}$. Let $\mathbf{u}, \mathbf{u}_{1} \in \mathcal{A}_{p}$ be such that $\mathbf{u}=\mathbf{u}_{1}$ a.e. and $\operatorname{det} D \mathbf{u}=1$ a.e. Let $\mathbf{v}, \mathbf{v}_{1} \in W^{1, q}\left(\Omega, \mathbb{R}^{d}\right)$ satisfy $\mathbf{v}=\mathbf{v}_{1}$ a.e. Given $B \in \mathcal{U}_{\mathbf{u}}^{\text {in }} \cap \mathcal{U}_{\mathbf{u}_{1}}^{\text {in }}$, define $\mathbf{w}:=\mathbf{v} \circ\left(\left.\mathbf{u}\right|_{B}\right)^{-1}$ and $\mathbf{w}_{1}:=\mathbf{v}_{1} \circ\left(\left.\mathbf{u}_{1}\right|_{B}\right)^{-1}$. Then $\left(\left.\mathbf{u}\right|_{B}\right)^{-1}=\left(\left.\mathbf{u}_{1}\right|_{B}\right)^{-1}$ a.e., $\mathbf{w}=\mathbf{w}_{1}$ a.e.,

$$
\mathbf{w} \in W^{1,1}\left(\operatorname{im}_{\mathrm{T}}(\mathbf{u}, B), \mathbb{R}^{d}\right) \quad \text { and } \quad D \mathbf{w}=\left(D \mathbf{v} \circ\left(\left.\mathbf{u}\right|_{B}\right)^{-1}\right) D\left(\left.\mathbf{u}\right|_{B}\right)^{-1} \text { a.e. }
$$

Proof. Let $\Omega_{0}$ be the set of Definition 2.11 corresponding to $\mathbf{u}$, and $\Omega_{1}$ the one corresponding to $\mathbf{u}_{1}$. Let $\Omega^{\prime}$ be the set where $\mathbf{u}$ and $\mathbf{u}_{1}$ coincide. By Definition 5.2, it is easy to see that

$$
\left(\left.\mathbf{u}\right|_{B}\right)^{-1}=\left(\left.\mathbf{u}_{1}\right|_{B}\right)^{-1} \text { in } \operatorname{im}_{\mathrm{G}}\left(\mathbf{u}, B \cap \Omega^{\prime}\right) \cap \operatorname{im}_{\mathrm{G}}\left(\mathbf{u}_{1}, B \cap \Omega^{\prime}\right) \cap \operatorname{im}_{\mathrm{T}}(\mathbf{u}, B) \cap \operatorname{im}_{\mathrm{T}}\left(\mathbf{u}_{1}, B\right) .
$$

By Remark 2.19 and Convention 2.14,

$$
\operatorname{im}_{\mathrm{T}}(\mathbf{u}, B)=\operatorname{im}_{\mathrm{T}}\left(\mathbf{u}_{1}, B\right)
$$

whereas by (5.1) and Lemma 2.8 a),

$$
\operatorname{im}_{\mathrm{T}}(\mathbf{u}, B)=\operatorname{im}_{\mathrm{G}}(\mathbf{u}, B)=\operatorname{im}_{\mathrm{G}}\left(\mathbf{u}, B \cap \Omega_{1} \cap \Omega^{\prime}\right)=\operatorname{im}_{\mathrm{G}}\left(\mathbf{u}_{1}, B \cap \Omega_{0} \cap \Omega^{\prime}\right)=\operatorname{im}_{\mathrm{G}}\left(\mathbf{u}_{1}, B\right)=\operatorname{im}_{\mathrm{T}}\left(\mathbf{u}_{1}, B\right) \text { a.e. }
$$

Therefore, $\left(\left.\mathbf{u}\right|_{B}\right)^{-1}=\left(\left.\mathbf{u}_{1}\right|_{B}\right)^{-1}$ a.e.

Now let $\Omega^{\prime \prime}$ be the set where $\mathbf{v}$ and $\mathbf{v}_{1}$ coincide. Then $\mathbf{w}=\mathbf{w}_{1}$ in

$$
G:=\left\{\mathbf{y} \in \operatorname{im}_{\mathrm{G}}\left(\mathbf{u}, B \cap \Omega^{\prime}\right) \cap \operatorname{im}_{\mathrm{G}}\left(\mathbf{u}_{1}, B \cap \Omega^{\prime}\right) \cap \operatorname{im}_{\mathrm{T}}(\mathbf{u}, B):\left(\left.\mathbf{u}\right|_{B}\right)^{-1}(\mathbf{y}) \in \Omega^{\prime \prime}\right\},
$$


but by (7.16), (7.17) and Proposition 2.7,

$$
\mathcal{L}^{n}(G) \geq \mathcal{L}^{n}\left(\left\{\mathbf{y} \in \operatorname{im}_{\mathrm{G}}(\mathbf{u}, B):\left(\left.\mathbf{u}\right|_{B}\right)^{-1}(\mathbf{y}) \in \Omega^{\prime \prime}\right\}\right)=\int_{B \cap \Omega^{\prime \prime}} \operatorname{det} D \mathbf{u}(\mathbf{x}) \mathrm{d} \mathbf{x}=\mathcal{L}^{n}\left(\operatorname{im}_{\mathrm{G}}(\mathbf{u}, B)\right),
$$

so $\mathbf{w}=\mathbf{w}_{1}$ a.e.

In order to prove the measurability of $\mathbf{w}$, it is enough to show, thanks to Lemma 2.9 , that the preimage under $\left(\left.\mathbf{u}\right|_{B}\right)^{-1}$ of a null set is also a null set, so by Lemma $2.8 c$ ) it suffices to show that the matrix $D\left(\left.\mathbf{u}\right|_{B}\right)^{-1}(\mathbf{y})$ is invertible for a.e. $\mathbf{y} \in \operatorname{im}_{\mathrm{T}}(\mathbf{u}, B)$, and this fact is a consequence of Proposition 5.3. Analogously, the function $\left(D \mathbf{v} \circ\left(\left.\mathbf{u}\right|_{B}\right)^{-1}\right) D\left(\left.\mathbf{u}\right|_{B}\right)^{-1}$ is measurable. In addition, by Proposition 2.7,

$$
\int_{\operatorname{im}_{\mathrm{T}}(\mathbf{u}, B)}|\mathbf{w}(\mathbf{y})| \mathrm{d} \mathbf{y}=\int_{B}|\mathbf{v}(\mathbf{x})| \mathrm{d} \mathbf{x}
$$

and, using Hölder's inequality,

$$
\begin{aligned}
\int_{\operatorname{im}_{\mathrm{T}}(\mathbf{u}, B)}\left|D \mathbf{v}\left(\left.\mathbf{u}\right|_{B}\right)^{-1}(\mathbf{y}) D\left(\left.\mathbf{u}\right|_{B}\right)^{-1}(\mathbf{y})\right| \mathrm{d} \mathbf{y} & =\int_{B}\left|D \mathbf{v}(\mathbf{x}) D\left(\left.\mathbf{u}\right|_{B}\right)^{-1}(\mathbf{u}(\mathbf{x}))\right| \mathrm{d} \mathbf{x} \\
& =\int_{B}|D \mathbf{v}(\mathbf{x}) \operatorname{adj} D \mathbf{u}(\mathbf{x})| \mathrm{d} \mathbf{x} \\
& \leq c_{n}\|D \mathbf{v}\|_{L^{q}\left(B, \mathbb{R}^{d \times n}\right)}\|D \mathbf{u}\|_{L^{p}\left(B, \mathbb{R}^{n \times n}\right)}^{n-1}
\end{aligned}
$$

for some constant $c_{n}>0$ depending on $n$, so

$$
\mathbf{w} \in L^{1}\left(\operatorname{im}_{\mathrm{T}}(\mathbf{u}, B), \mathbb{R}^{d}\right) \quad \text { and } \quad\left(D \mathbf{v} \circ\left(\left.\mathbf{u}\right|_{B}\right)^{-1}\right) D\left(\left.\mathbf{u}\right|_{B}\right)^{-1} \in L^{1}\left(\operatorname{im}_{\mathrm{T}}(\mathbf{u}, B), \mathbb{R}^{d \times n}\right) .
$$

Now let $\left\{\overline{\mathbf{v}}_{i}\right\}_{i \in \mathbb{N}}$ be a sequence in $C^{1}\left(\bar{B}, \mathbb{R}^{d}\right)$ converging to $\mathbf{v}$ in $W^{1, q}\left(B, \mathbb{R}^{d}\right)$. By the chain rule (see, e.g., [29, Th. 4 in Sect. 4.2.2]), for each $i \in \mathbb{N}$, the function $\overline{\mathbf{w}}_{i}:=\overline{\mathbf{v}}_{i} \circ\left(\left.\mathbf{u}\right|_{B}\right)^{-1}$ is in $W^{1,1}\left(\operatorname{im}_{\mathrm{T}}(\mathbf{u}, B), \mathbb{R}^{d}\right)$ and $D \overline{\mathbf{w}}_{i}=\left(D \overline{\mathbf{v}}_{i} \circ\left(\left.\mathbf{u}\right|_{B}\right)^{-1}\right) D\left(\left.\mathbf{u}\right|_{B}\right)^{-1}$ a.e. As in (7.18)-(7.19), we obtain

$$
\int_{\operatorname{im}_{\mathrm{T}}(\mathbf{u}, B)}\left|\overline{\mathbf{w}}_{i}(\mathbf{y})-\mathbf{w}(\mathbf{y})\right| \mathrm{d} \mathbf{y}=\int_{B}\left|\overline{\mathbf{v}}_{i}(\mathbf{x})-\mathbf{v}(\mathbf{x})\right| \mathrm{d} \mathbf{x}
$$

and

$$
\begin{aligned}
\int_{\operatorname{im}_{\mathrm{T}}(\mathbf{u}, B)}\left|D \overline{\mathbf{w}}_{i}(\mathbf{y})-D \mathbf{v}\left(\left.\mathbf{u}\right|_{B}\right)^{-1}(\mathbf{y}) D\left(\left.\mathbf{u}\right|_{B}\right)^{-1}(\mathbf{y})\right| \mathrm{d} \mathbf{y} & =\int_{B}\left|\left(D \overline{\mathbf{v}}_{i}(\mathbf{x})-D \mathbf{v}(\mathbf{x})\right) \operatorname{adj} D \mathbf{u}(\mathbf{x})\right| \mathrm{d} \mathbf{x} \\
& \leq c_{n}\left\|D \overline{\mathbf{v}}_{i}-D \mathbf{v}\right\|_{L^{q}\left(B, \mathbb{R}^{d \times n}\right)}\|D \mathbf{u}\|_{L^{p}\left(B, \mathbb{R}^{n \times n}\right)}^{n-1} .
\end{aligned}
$$

Thus,

$$
\overline{\mathbf{w}}_{i} \rightarrow \mathbf{w} \text { in } L^{1}\left(\mathrm{im}_{\mathrm{T}}(\mathbf{u}, B), \mathbb{R}^{d}\right) \quad \text { and } \quad D \overline{\mathbf{w}}_{i} \rightarrow\left(D \mathbf{v} \circ\left(\left.\mathbf{u}\right|_{B}\right)^{-1}\right) D\left(\left.\mathbf{u}\right|_{B}\right)^{-1} \text { in } L^{1}\left(\operatorname{im}_{\mathrm{T}}(\mathbf{u}, B), \mathbb{R}^{d \times n}\right)
$$

as $i \rightarrow \infty$, which allows us to conclude the proof.

The following proposition is a lower semicontinuity result for functionals of the form (7.15). This kind of functional will not be given an application in Section 8 but it may be useful in some contexts. As a matter of fact, it provides a partial answer in the affirmative to questions posed in [32, Eq. (44) and Rk. 4.2.4]; in particular, it extends [32, Th. 4.1] from $p \geq n$ to $p>n-1$.

Proposition 7.3. Let $p>n-1, q>\left(\frac{p}{n-1}\right)^{\prime}, r:=\frac{p q}{p+(n-1) q}$ and $d \in \mathbb{N}$. Let $\left\{\mathbf{u}_{j}\right\}_{j \in \mathbb{N}}$ be a sequence in $\mathcal{A}_{p}$ such that $\operatorname{det} D \mathbf{u}_{j}=1$ a.e. for all $j \in \mathbb{N}$, and $\mathbf{u}_{j} \rightarrow \mathbf{u}$ in $W^{1, p}\left(\Omega, \mathbb{R}^{n}\right)$ as $j \rightarrow \infty$, for some $\mathbf{u} \in W^{1, p}\left(\Omega, \mathbb{R}^{n}\right)$. Let also $\left\{\mathbf{v}_{j}\right\}_{j \in \mathbb{N}}$ be a sequence in $W^{1, q}\left(\Omega, \mathbb{R}^{d}\right)$. Consider a quasiconvex function $W: \mathbb{R}^{d \times n} \rightarrow[0, \infty)$ for which there exists $c>0$ such that

$$
W(\mathbf{F}) \leq c\left(1+|\mathbf{F}|^{r}\right), \quad \mathbf{F} \in \mathbb{R}^{d \times n} .
$$

The following assertions hold: 
a) If $D \mathbf{v}_{j}\left(D \mathbf{u}_{j}\right)^{-1} \rightarrow \mathbf{L}$ in $L^{r}\left(\Omega, \mathbb{R}^{d \times n}\right)$ as $j \rightarrow \infty$ for some $\mathbf{L} \in L^{r}\left(\Omega, \mathbb{R}^{d \times n}\right)$, then

$$
\int_{\Omega} W(\mathbf{L}(\mathbf{x})) \mathrm{d} \mathbf{x} \leq \liminf _{j \rightarrow \infty} \int_{\Omega} W\left(D \mathbf{v}_{j}(\mathbf{x}) D \mathbf{u}_{j}(\mathbf{x})^{-1}\right) \mathrm{d} \mathbf{x} .
$$

b) If $\mathbf{v}_{j} \rightarrow \mathbf{v}$ in $W^{1, q}\left(\Omega, \mathbb{R}^{d}\right)$ as $j \rightarrow \infty$ for some $\mathbf{v} \in W^{1, q}\left(\Omega, \mathbb{R}^{d \times n}\right)$, then

$$
D \mathbf{v}_{j}\left(D \mathbf{u}_{j}\right)^{-1} \rightarrow D \mathbf{v}(D \mathbf{u})^{-1} \text { in } L^{r}\left(\Omega, \mathbb{R}^{d \times n}\right) \quad \text { as } j \rightarrow \infty .
$$

Proof. Notice that, by Proposition 6.1 , $\operatorname{det} D \mathbf{u}=1$ a.e. and $\mathbf{u} \in \mathcal{A}_{p}$. Note also that $r>1$.

We start by proving a). For a subsequence, we can assume that the lim inf of the right-hand side of (7.20) is actually a limit, and that convergence (6.3) holds. Let $\left\{B_{k}\right\}_{k \in \mathbb{N}}$ be the family of Theorem $6.3 b$ ) and consider the subsequence therein. By Lemma 7.2 , for each $j, k \in \mathbb{N}$, the function $\mathbf{w}_{j k}:=\mathbf{v}_{j} \circ\left(\left.\mathbf{u}_{j}\right|_{B_{k}}\right)^{-1}$ satisfies

$$
\mathbf{w}_{j k} \in W^{1,1}\left(\operatorname{im}_{\mathrm{T}}\left(\mathbf{u}_{j}, B_{k}\right), \mathbb{R}^{d}\right) \quad \text { and } \quad D \mathbf{w}_{j}=\left(D \mathbf{v}_{j} \circ\left(\left.\mathbf{u}_{j}\right|_{B_{k}}\right)^{-1}\right) D\left(\left.\mathbf{u}_{j}\right|_{B_{k}}\right)^{-1} \text { a.e. }
$$

In addition,

$$
\sup _{j \in \mathbb{N}} \int_{\operatorname{im}_{\mathrm{T}}\left(\mathbf{u}_{j}, B_{k}\right)}\left|D \mathbf{w}_{j k}(\mathbf{y})\right|^{r} \mathrm{~d} \mathbf{y}=\sup _{j \in \mathbb{N}} \int_{B_{k}}\left|D \mathbf{v}_{j}(\mathbf{x}) D \mathbf{u}_{j}(\mathbf{x})^{-1}\right|^{r} \mathrm{~d} \mathbf{x}<\infty .
$$

Consider a smooth connected open set $V_{k} \subset \subset \operatorname{im}_{\mathrm{T}}\left(\mathbf{u}, B_{k}\right)$. By Lemma $\left.3.6 a\right)$, for a subsequence in $j$, we have $V_{k} \subset \operatorname{im}_{\mathrm{T}}\left(\mathbf{u}_{j}, B_{k}\right)$ for all $j \in \mathbb{N}$. Then, thanks to (7.22) and the Poincaré-Wirtinger inequality, there is $\mathbf{c}_{j k} \in \mathbb{R}^{n}$ such that $\left\{\mathbf{w}_{j k}-\mathbf{c}_{j k}\right\}_{j \in \mathbb{N}}$ is bounded in $W^{1, r}\left(V_{k}, \mathbb{R}^{n}\right)$, so there exists $\mathbf{w}_{k} \in W^{1, r}\left(V_{k}, \mathbb{R}^{n}\right)$ such that, for a subsequence,

$$
\mathbf{w}_{j k}-\mathbf{c}_{j k} \rightarrow \mathbf{w}_{k} \quad \text { in } W^{1, r}\left(V_{k}, \mathbb{R}^{n}\right) \text { as } j \rightarrow \infty .
$$

Let $\varphi \in C_{c}\left(V_{k}\right)$. The proof of Theorem $6.3 c$ ) shows that the analogue of (6.4) holds, with $B_{k}$ replacing $B$ and $V_{k}$ replacing $V$. Then, thanks to the assumption in $a$ ) we have

$$
\begin{aligned}
\lim _{j \rightarrow \infty} \int_{V_{k}} \varphi(\mathbf{y}) D \mathbf{w}_{j k}(\mathbf{y}) \mathrm{d} \mathbf{y} & =\lim _{j \rightarrow \infty} \int_{\left(\left.\mathbf{u}_{j}\right|_{B_{k}}\right)^{-1}\left(V_{k} \cap \operatorname{im}_{\mathrm{G}}\left(\mathbf{u}_{j}, B_{k}\right)\right)} \varphi\left(\mathbf{u}_{j}(\mathbf{x})\right) D \mathbf{v}_{j}(\mathbf{x}) D \mathbf{u}_{j}(\mathbf{x})^{-1} \mathrm{~d} \mathbf{x} \\
& =\int_{\left(\left.\mathbf{u}\right|_{B_{k}}\right)^{-1}\left(V_{k} \cap \operatorname{im}_{\mathrm{G}}\left(\mathbf{u}, B_{k}\right)\right)} \varphi(\mathbf{u}(\mathbf{x})) \mathbf{L}(\mathbf{x}) \mathrm{d} \mathbf{x}=\int_{V_{k}} \varphi(\mathbf{y}) \mathbf{L}\left(\left(\left.\mathbf{u}\right|_{B_{k}}\right)^{-1}(\mathbf{y})\right) \mathrm{d} \mathbf{y} .
\end{aligned}
$$

This shows that $D \mathbf{w}_{j k} \stackrel{*}{\rightarrow} \mathbf{L} \circ\left(\left.\mathbf{u}\right|_{B_{k}}\right)^{-1}$ in $\mathcal{M}\left(V_{k}, \mathbb{R}^{d \times n}\right)$ as $j \rightarrow \infty$. Comparing this with (7.23) we find that $D \mathbf{w}_{k}=\mathbf{L} \circ\left(\left.\mathbf{u}\right|_{B_{k}}\right)^{-1}$ a.e. in $V_{k}$. By the lower semicontinuity theorem for quasiconvex functions (see, e.g., $[17$, Th. 8.4]), thanks to (7.23) we have

$$
\int_{V_{k}} W\left(\mathbf{L} \circ\left(\left.\mathbf{u}\right|_{B_{k}}\right)^{-1}\right) \mathrm{d} \mathbf{y}=\int_{V_{k}} W\left(D \mathbf{w}_{k}\right) \mathrm{d} \mathbf{y} \leq \liminf _{j \rightarrow \infty} \int_{V_{k}} W\left(D \mathbf{w}_{j k}\right) \mathrm{d} \mathbf{y} \leq \liminf _{j \rightarrow \infty} \int_{\operatorname{im}_{\mathrm{T}}\left(\mathbf{u}_{j}, B_{k}\right)} W\left(D \mathbf{w}_{j k}\right) \mathrm{d} \mathbf{y} .
$$

As $V_{k}$ is arbitrary in $\operatorname{im}_{\mathrm{T}}\left(\mathbf{u}_{j}, B_{k}\right)$, by monotone convergence, we conclude that

$$
\int_{\operatorname{im}_{\mathrm{T}}\left(\mathbf{u}_{j}, B_{k}\right)} W\left(\mathbf{L} \circ\left(\left.\mathbf{u}\right|_{B_{k}}\right)^{-1}\right) \mathrm{d} \mathbf{y} \leq \liminf _{j \rightarrow \infty} \int_{\operatorname{im}_{\mathrm{T}}\left(\mathbf{u}_{j}, B_{k}\right)} W\left(D \mathbf{w}_{j k}\right) \mathrm{d} \mathbf{y}
$$

whereas by a change of variables, we get

$$
\int_{B_{k}} W(\mathbf{L}(\mathbf{x})) \mathrm{d} \mathbf{x} \leq \liminf _{j \rightarrow \infty} \int_{B_{k}} W\left(D \mathbf{v}_{j}(\mathbf{x}) D \mathbf{u}_{j}(\mathbf{x})^{-1}\right) \mathrm{d} \mathbf{x} .
$$

Summing in $k \in \mathbb{N}$ the above inequality, we conclude (7.20).

In order to prove b), observe that, since $\left(D \mathbf{u}_{j}\right)^{-1}=\operatorname{adj} D \mathbf{u}_{j}$ for all $j \in \mathbb{N}$, the sequence $\left\{\left(D \mathbf{u}_{j}\right)^{-1}\right\}_{j \in \mathbb{N}}$ is bounded in $L^{\frac{p}{n-1}}\left(\Omega, \mathbb{R}^{n \times n}\right)$, so, thanks to Hölder's inequality, $\left\{D \mathbf{v}_{j}\left(D \mathbf{u}_{j}\right)^{-1}\right\}_{j \in \mathbb{N}}$ is bounded in $L^{r}\left(\Omega, \mathbb{R}^{d \times n}\right)$. Moreover, using the fact that the distributional divergence of the columns of adj $D \mathbf{u}_{j}$ is zero (this is Piola's identity for Sobolev maps, see, e.g., [6, Lemma 6.1] or [38, Prop. 3.2.4.1]), by applying componentwise the div-curl lemma (see, e.g., [64]) we obtain the conclusion of $b$ ). 


\subsection{Div-quasiconvex functionals under incompressibility}

As is well-known (see, e.g., [17, Sect. 8.2]), quasiconvexity of $W$ is a necessary and sufficient condition for the lower semicontinuity in $W^{1, p}\left(\Omega, \mathbb{R}^{n}\right)$ of functionals of the form

$$
\mathbf{u} \mapsto \int_{\Omega} W(D \mathbf{u}(\mathbf{x})) \mathrm{d} \mathbf{x} .
$$

In this subsection we show that in the set $\left\{\mathbf{u} \in \mathcal{A}_{p}\right.$ : $\operatorname{det} D \mathbf{u}=1$ a.e. $\}$ the lower semicontinuity also holds when the integrand $W: \mathbb{R}^{n \times n} \rightarrow \mathbb{R}$ is Div-quasiconvex on columns.

Definition 7.4. A Borel function $W: \mathbb{R}^{n \times n} \rightarrow \mathbb{R}$ is said to be Div-quasiconvex on columns if

$$
W(\mathbf{F}) \leq \int_{(0,1)^{n}} W(\mathbf{F}+\mathbf{\Phi}(\mathbf{z})) \mathrm{d} \mathbf{z}
$$

for all $\mathbf{F} \in \mathbb{R}^{n \times n}$ and all $(0,1)^{n}$-periodic functions $\boldsymbol{\Phi} \in C^{\infty}\left(\mathbb{R}^{n}, \mathbb{R}^{n \times n}\right)$ such that Div $\boldsymbol{\Phi}=\mathbf{0}$ and

$$
\int_{(0,1)^{n}} \mathbf{\Phi}(\mathbf{z}) \mathrm{d} \mathbf{z}=\mathbf{0} .
$$

Here and in the rest of the section, the operator Div acts as a divergence on each column.

We refer to Fonseca \& Müller [34] for the theory of $\mathcal{A}$-quasiconvexity, of which Div-quasiconvexity is a particular case, and to $[3,66,65]$ for further results on Div-quasiconvexity.

We first show that the local inverse of $\mathbf{u}$ satisfies Piola's identity. Note that the standard proof (see, e.g., [6, Lemma 6.1] or [38, Prop. 3.2.4.1]) cannot be applied because of the low regularity of the inverse.

Lemma 7.5. Let $p>n-1, \mathbf{u} \in \mathcal{A}_{p}$ and $U \in \mathcal{U}_{\mathbf{u}}^{N \text {,in }}$. Then $\operatorname{Div} \operatorname{adj} D\left(\left.\mathbf{u}\right|_{U}\right)^{-1}=\mathbf{0}$ in the sense of distributions.

Proof. We first note that $\operatorname{cof} D\left(\left.\mathbf{u}\right|_{U}\right)^{-1} \in L^{1}\left(\operatorname{im}_{\mathrm{T}}(\mathbf{u}, U), \mathbb{R}^{n \times n}\right)$ since, as in (6.8),

$$
\int_{\operatorname{im}_{\mathrm{T}}(\mathbf{u}, U)}\left|\operatorname{cof} D\left(\left.\mathbf{u}\right|_{U}\right)^{-1}(\mathbf{y})\right| \mathrm{d} \mathbf{y}=\int_{U}|D \mathbf{u}(\mathbf{x})| \mathrm{d} \mathbf{x} .
$$

Let $\varphi \in C_{c}^{1}\left(\operatorname{im}_{\mathrm{T}}(\mathbf{u}, U)\right)$. By the chain rule (e.g., [29, Th. 4.2.2.4]), $\varphi \circ \mathbf{u} \in W^{1, p}(\Omega)$ and

$$
D(\varphi \circ \mathbf{u})(\mathbf{x})=D \mathbf{u}(\mathbf{x})^{T} D \varphi(\mathbf{u}(\mathbf{x})), \quad \text { a.e. } \mathbf{x} \in \Omega .
$$

As $\operatorname{spt} \varphi$ is compact and contained $\operatorname{in}_{\mathrm{T}}(\mathbf{u}, U)$, thanks to Lemma 5.18 a) there exists $t>0$ such that $U_{t} \in \mathcal{U}_{\mathbf{u}}^{N}$ and $\operatorname{spt} \varphi \subset \operatorname{im}_{\mathrm{T}}\left(\mathbf{u}, U_{t}\right)$. It follows from (5.1), Lemma $2.8 c$ ) and the a.e.-invertibility of $\mathbf{u}$ in $U$ that $\mathbf{u}(\mathbf{x}) \notin \operatorname{im}_{\mathrm{T}}\left(\mathbf{u}, U_{t}\right)$ for a.e. $\mathbf{x} \in U \backslash U_{t}$. Therefore, $\varphi(\mathbf{u}(\mathbf{x}))=0$ for a.e. $\mathbf{x} \in U \backslash U_{t}$. Thus, an integration by parts yields (see, e.g., [29, Th. 4.3.1])

$$
\int_{U} D(\varphi \circ \mathbf{u})(\mathbf{x}) \mathrm{d} \mathbf{x}=\mathbf{0} .
$$

Owing to (7.25) and Propositions 2.7 and 5.3, we obtain

$$
\mathbf{0}=\int_{U} D \mathbf{u}(\mathbf{x})^{T} D \varphi(\mathbf{u}(\mathbf{x})) \mathrm{d} \mathbf{x}=\int_{\operatorname{im}_{\mathrm{T}}(\mathbf{u}, U)} \operatorname{cof} D\left(\left.\mathbf{u}\right|_{U}\right)^{-1}(\mathbf{y}) D \varphi(\mathbf{y}) \mathrm{d} \mathbf{y}
$$

and the conclusion follows.

The following lower semicontinuity result is achieved due, again, to the possibility of working in the deformed configuration. As far as we know, it constitutes the first general lower semicontinuity result for integrands of the form (7.24) under Div-quasiconvexity and the additional incompressibility constraint. 
Proposition 7.6. Let $p>n-1$. Let $\left\{\mathbf{u}_{j}\right\}_{j \in \mathbb{N}}$ be a sequence in $\mathcal{A}_{p}$ such that $\operatorname{det} D \mathbf{u}_{j}=1$ a.e. for all $j \in \mathbb{N}$ and $\mathbf{u}_{j} \rightarrow \mathbf{u}$ in $W^{1, p}\left(\Omega, \mathbb{R}^{n}\right)$ as $j \rightarrow \infty$, for some $\mathbf{u} \in W^{1, p}\left(\Omega, \mathbb{R}^{n}\right)$. Consider a continuous function $W: \mathbb{R}^{n \times n} \rightarrow[0, \infty)$ that is Div-quasiconvex on columns and for which there exists $c>0$ with

$$
W(\mathbf{F}) \leq c\left(1+|\mathbf{F}|^{p}\right), \quad \mathbf{F} \in \mathbb{R}^{n \times n} .
$$

Then

$$
\int_{\Omega} W(D \mathbf{u}(\mathbf{x})) \mathrm{d} \mathbf{x} \leq \liminf _{j \rightarrow \infty} \int_{\Omega} W\left(D \mathbf{u}_{j}(\mathbf{x})\right) \mathrm{d} \mathbf{x} .
$$

Proof. By Proposition 6.1, $\operatorname{det} D \mathbf{u}=1$ a.e. and $\mathbf{u} \in \mathcal{A}_{p}$. For a subsequence, we can assume that the liminf of the right-hand side of (7.26) is actually a limit.

Let $\left\{B_{k}\right\}_{k \in \mathbb{N}}$ be the family of Theorem $6.3 b$ ), consider the subsequence therein and fix $k \in \mathbb{N}$. Thanks to Proposition 5.3, for each $j \in \mathbb{N}$,

$$
\left(\left.\mathbf{u}_{j}\right|_{B_{k}}\right)^{-1} \in W^{1,1}\left(\operatorname{im}_{\mathrm{T}}\left(\mathbf{u}_{j}, B_{k}\right), \mathbb{R}^{n}\right) \quad \text { and } \quad \operatorname{adj} D\left(\left.\mathbf{u}_{j}\right|_{B_{k}}\right)^{-1}=\left(D\left(\left.\mathbf{u}_{j}\right|_{B_{k}}\right)^{-1}\right)^{-1}=D \mathbf{u}_{j} \circ\left(\left.\mathbf{u}_{j}\right|_{B_{k}}\right)^{-1} \text { a.e. }
$$

and analogously for $\mathbf{u}$ replacing $\mathbf{u}_{j}$. In addition, by Proposition 2.7,

$$
\sup _{j \in \mathbb{N}} \int_{\operatorname{im}_{\mathrm{T}}\left(\mathbf{u}_{j}, B_{k}\right)}\left|\operatorname{adj} D\left(\left.\mathbf{u}_{j}\right|_{B_{k}}\right)^{-1}(\mathbf{y})\right|^{p} \mathrm{~d} \mathbf{y}=\sup _{j \in \mathbb{N}} \int_{B_{k}}\left|D \mathbf{u}_{j}(\mathbf{x})\right|^{p} \mathrm{~d} \mathbf{x}<\infty .
$$

Let $V_{k} \subset \subset \operatorname{im}_{\mathrm{T}}\left(\mathbf{u}, B_{k}\right)$ be open. By Lemma 3.6, for $j$ large enough we have $V_{k} \subset \operatorname{im}_{\mathrm{T}}\left(\mathbf{u}_{j}, B_{k}\right)$ and, by Theorem $6.3 c$ ),

$$
\operatorname{adj} D\left(\left.\mathbf{u}_{j}\right|_{B_{k}}\right)^{-1} \rightarrow \operatorname{adj} D\left(\left.\mathbf{u}\right|_{B_{k}}\right)^{-1} \text { in } L^{1}\left(V_{k}, \mathbb{R}^{n \times n}\right) \text { as } j \rightarrow \infty .
$$

Moreover, bound (7.27) implies that the above convergence also holds weakly in $L^{p}\left(V_{k}, \mathbb{R}^{n \times n}\right)$. Using Lemma 7.5 and the semicontinuity result stated in [34, Th. 3.7], we have that

$$
\int_{V_{k}} W\left(\operatorname{adj} D\left(\left.\mathbf{u}\right|_{B_{k}}\right)^{-1}\right) \mathrm{d} \mathbf{y} \leq \liminf _{j \rightarrow \infty} \int_{V_{k}} W\left(\operatorname{adj} D\left(\left.\mathbf{u}_{j}\right|_{B_{k}}\right)^{-1}\right) \mathrm{d} \mathbf{y} \leq \liminf _{j \rightarrow \infty} \int_{\operatorname{im}_{\mathrm{T}}\left(\mathbf{u}_{j}, B_{k}\right)} W\left(\operatorname{adj} D\left(\left.\mathbf{u}_{j}\right|_{B_{k}}\right)^{-1}\right) \mathrm{d} \mathbf{y} .
$$

Since $V_{k}$ was arbitrary in $\operatorname{im}_{\mathrm{T}}\left(\mathbf{u}, B_{k}\right)$, we conclude, by monotone convergence, that

$$
\int_{\operatorname{im}_{\mathrm{T}}\left(\mathbf{u}, B_{k}\right)} W\left(\operatorname{adj} D\left(\left.\mathbf{u}\right|_{B_{k}}\right)^{-1}(\mathbf{y})\right) \mathrm{d} \mathbf{y} \leq \liminf _{j \rightarrow \infty} \int_{\operatorname{im}_{\mathrm{T}}\left(\mathbf{u}_{j}, B_{k}\right)} W\left(\operatorname{adj} D\left(\left.\mathbf{u}_{j}\right|_{B_{k}}\right)^{-1}(\mathbf{y})\right) \mathrm{d} \mathbf{y} .
$$

A change of variables yields

$$
\int_{B_{k}} W(D \mathbf{u}(\mathbf{x})) \mathrm{d} \mathbf{x} \leq \liminf _{j \rightarrow \infty} \int_{B_{k}} W\left(D \mathbf{u}_{j}(\mathbf{x})\right) \mathrm{d} \mathbf{x} .
$$

The proof is concluded by summing in $k \in \mathbb{N}$ the above inequality.

\subsection{Polyconvex functionals involving a composition of maps}

In this subsection we analyze functionals of the form

$$
\int_{\Omega} W_{0}(D \mathbf{u}(\mathbf{x}), \mathbf{w}(\mathbf{u}(\mathbf{x}))) \mathrm{d} \mathbf{x}
$$

where $W_{0}$ is polyconvex in its first argument. We recall that a Borel function $f: \mathbb{R}^{n \times n} \rightarrow \mathbb{R} \cup\{\infty\}$ is polyconvex if it can be expressed as a convex functions of the minors of its argument (see, e.g., [17, Def. 5.1]); we say that an $f: \mathbb{R}_{+}^{n \times n} \rightarrow \mathbb{R} \cup\{\infty\}$ is polyconvex if its extension by $\infty$ to $\mathbb{R}^{n \times n} \backslash \mathbb{R}_{+}^{n \times n}$ is polyconvex in the sense above.

The main difficulty for proving lower semicontinuity relies in the composition $\mathbf{w} \circ \mathbf{u}$, since in general the weak convergences of $\left\{\mathbf{w}_{j}\right\}_{j \in \mathbb{N}}$ and $\left\{\mathbf{u}_{j}\right\}_{j \in \mathbb{N}}$ do not imply the weak convergence of $\left\{\mathbf{w}_{j} \circ \mathbf{u}_{j}\right\}_{j \in \mathbb{N}}$. We overcome this obstacle by working locally in balls where $\mathbf{u}$ and all $\mathbf{u}_{j}$ are invertible: a change of variables will then allow us to work in the deformed configuration, where no composition of maps is involved.

We first show that (7.28) is well defined. 
Lemma 7.7. Let $p>n-1, d \in \mathbb{N}$ and $q:=\left(\frac{p}{n-1}\right)^{\prime}$. Let $\mathbf{u}, \mathbf{u}_{1} \in \mathcal{A}_{p}$ be such that $\mathbf{u}=\mathbf{u}_{1}$ a.e. Let $\mathbf{w}, \mathbf{w}_{1}: \operatorname{im}_{\mathrm{T}}(\mathbf{u}, \Omega) \rightarrow \mathbb{R}^{d}$ be measurable maps satisfying $\mathbf{w}=\mathbf{w}_{1}$ a.e. Then $\mathbf{w} \circ \mathbf{u}$ is measurable and $\mathbf{w} \circ \mathbf{u}=\mathbf{w}_{1} \circ \mathbf{u}_{1}$ a.e.

Proof. Thanks to Lemma 2.9, the map $\mathbf{w} \circ \mathbf{u}$ is measurable provided that the preimage of a set of measure zero under $\mathbf{u}$ is a set of measure zero, and this last fact is a consequence of Lemma $2.8 c$ ).

Let $A$ be the set where $\mathbf{u}$ and $\mathbf{u}_{1}$ coincide, and let $B$ be the set where $\mathbf{w}$ and $\mathbf{w}_{1}$ coincide. Then $\mathbf{w} \circ \mathbf{u}$ and $\mathbf{w}_{1} \circ \mathbf{u}_{1}$ coincide in $\{\mathbf{x} \in A: \mathbf{u}(\mathbf{x}) \in B\}$, which is a set of full measure in $\Omega$ again because the preimage of a set of measure zero under $\mathbf{u}$ is a set of measure zero.

The lower semicontinuity result is as follows. As in Proposition 7.1, its proof is inspired in [11], but we only require $p>n-1$ and no incompressibility constraint is needed.

Proposition 7.8. Let $p>n-1$ and $d \in \mathbb{N}$. For each $j \in \mathbb{N}$, let $\mathbf{u}, \mathbf{u}_{j} \in \mathcal{A}_{p}$ and let

$$
\mathbf{w}: \operatorname{im}_{\mathrm{T}}(\mathbf{u}, \Omega) \rightarrow \mathbb{R}^{d} \quad \text { and } \quad \mathbf{w}_{j}: \operatorname{im}_{\mathrm{T}}\left(\mathbf{u}_{j}, \Omega\right) \rightarrow \mathbb{R}^{d}
$$

be measurable such that

$$
\mathbf{u}_{j} \rightarrow \mathbf{u} \text { in } W^{1, p}\left(\Omega, \mathbb{R}^{n}\right) \quad \text { and } \quad \mathbf{w}_{j} \rightarrow \mathbf{w} \text { a.e. as } j \rightarrow \infty .
$$

Suppose, in addition, that there exists a Borel function $h:(0, \infty) \rightarrow[0, \infty)$ such that

$$
\lim _{t \searrow 0} h(t)=\infty
$$

and for any compact $K \subset \Omega$,

$$
\sup _{j \in \mathbb{N}} \int_{K} h\left(\operatorname{det} D \mathbf{u}_{j}(\mathbf{x})\right) \mathrm{d} \mathbf{x}<\infty .
$$

Let $W_{0}: \mathbb{R}_{+}^{n \times n} \times \mathbb{R}^{d} \rightarrow[0, \infty)$ be a continuous function such that $W_{0}(\cdot, \mathbf{m})$ is polyconvex for all $\mathbf{m} \in \mathbb{R}^{d}$. Then

$$
\int_{\Omega} W_{0}(D \mathbf{u}(\mathbf{x}), \mathbf{w}(\mathbf{u}(\mathbf{x}))) \mathrm{d} \mathbf{x} \leq \liminf _{j \rightarrow \infty} \int_{\Omega} W_{0}\left(D \mathbf{u}_{j}(\mathbf{x}), \mathbf{w}_{j}\left(\mathbf{u}_{j}(\mathbf{x})\right)\right) \mathrm{d} \mathbf{x} .
$$

The convergence $\mathbf{w}_{j} \rightarrow \mathbf{w}$ of (7.29) is to be understood in the following sense: for a.e. $\mathbf{y} \in \operatorname{im}_{\mathrm{T}}(\mathbf{u}, \Omega)$ there exists $j_{\mathbf{y}} \in \mathbb{N}$ such that $\mathbf{y} \in \operatorname{im}_{\mathrm{T}}\left(\mathbf{u}_{j}, \Omega\right)$ for all $j \geq j_{\mathbf{y}}$ and the sequence $\left\{\mathbf{w}_{j}(\mathbf{y})\right\}_{j \geq j_{\mathbf{y}}}$ converges to $\mathbf{w}(\mathbf{y})$.

Proof. Consider a subsequence for which the lim inf of the right-hand side of (7.32) is actually a limit. Define the function $\hat{W}: \mathbb{R}_{+}^{n \times n} \times \mathbb{R}^{d} \rightarrow[0, \infty)$ as

$$
\hat{W}(\mathbf{F}, \mathbf{m}):=W_{0}\left(\mathbf{F}^{-1}, \mathbf{m}\right) \operatorname{det} \mathbf{F} .
$$

It was shown in [5, Th. 2.6] (see also [48, Prop. 1.1]) that $\hat{W}(\cdot, \mathbf{m})$ is polyconvex for all $\mathbf{m} \in \mathbb{R}^{d}$.

Let $\left\{B_{k}\right\}_{k \in \mathbb{N}}$ be the family of Theorem $6.3 \mathrm{~b}$ ), consider the subsequence therein, and fix $k \in \mathbb{N}$. Using (7.33), (5.1) and Proposition 2.7, we obtain that

$$
\int_{B_{k}} W_{0}(D \mathbf{u}(\mathbf{x}), \mathbf{w}(\mathbf{u}(\mathbf{x}))) \mathrm{d} \mathbf{x}=\int_{\operatorname{im}_{\mathrm{T}}\left(\mathbf{u}, B_{k}\right)} \hat{W}\left(D\left(\left.\mathbf{u}\right|_{B_{k}}\right)^{-1}(\mathbf{y}), \mathbf{w}(\mathbf{y})\right) \mathrm{d} \mathbf{y}
$$

and, for all $j \in \mathbb{N}$,

$$
\int_{B_{k}} W_{0}\left(D \mathbf{u}_{j}(\mathbf{x}), \mathbf{w}_{j}(\mathbf{u}(\mathbf{x}))\right) \mathrm{d} \mathbf{x}=\int_{\operatorname{im}_{\mathrm{T}}\left(\mathbf{u}_{j}, B_{k}\right)} \hat{W}\left(D\left(\left.\mathbf{u}_{j}\right|_{B_{k}}\right)^{-1}(\mathbf{y}), \mathbf{w}_{j}(\mathbf{y})\right) \mathrm{d} \mathbf{y} .
$$

Define $h_{1}:(0, \infty) \rightarrow \mathbb{R}$ as $h_{1}(t):=t h\left(\frac{1}{t}\right)$. Thanks to $(7.30)$,

$$
\lim _{t \rightarrow \infty} \frac{h_{1}(t)}{t}=\lim _{t \rightarrow \infty} h\left(\frac{1}{t}\right)=\lim _{t \searrow 0} h(t)=\infty .
$$


Let $V_{k} \subset \subset \operatorname{im}_{\mathrm{T}}\left(\mathbf{u}, B_{k}\right)$ be open. Then $V_{k} \subset \operatorname{im}_{\mathrm{T}}\left(\mathbf{u}_{j}, B_{k}\right)$ for $j \in \mathbb{N}$ large enough, thanks to Lemma 3.6. Using equality (5.1) and Proposition 2.7, we find that, for such $j$,

$$
\int_{V_{k}} h_{1}\left(\operatorname{det} D\left(\left.\mathbf{u}_{j}\right|_{B_{k}}\right)^{-1}(\mathbf{y})\right) \mathrm{d} \mathbf{y} \leq \int_{\operatorname{im}_{\mathrm{T}}\left(\mathbf{u}_{j}, B_{k}\right)} h_{1}\left(\operatorname{det} D\left(\left.\mathbf{u}_{j}\right|_{B_{k}}\right)^{-1}(\mathbf{y})\right) \mathrm{d} \mathbf{y}=\int_{B_{k}} h\left(\operatorname{det} D \mathbf{u}_{j}(\mathbf{x})\right) \mathrm{d} \mathbf{x} .
$$

Thanks to (7.31), (7.36) and de la Vallée-Poussin's criterion, the sequence $\left\{\operatorname{det} D\left(\left.\mathbf{u}_{j}\right|_{B}\right)^{-1}\right\}_{j \in \mathbb{N}}$ is equiintegrable in $V_{k}$. Therefore, by Theorem $6.3 c$ ), for any minor $M$ of the $\mathbb{R}^{n \times n}$ matrices,

$$
\begin{aligned}
& \left(\left.\mathbf{u}_{j}\right|_{B_{k}}\right)^{-1} \rightarrow\left(\left.\mathbf{u}\right|_{B_{k}}\right)^{-1} \quad \text { in } W^{1,1}\left(V_{k}, \mathbb{R}^{n}\right) \quad \text { and } \\
& M\left(D\left(\left.\mathbf{u}_{j}\right|_{B_{k}}\right)^{-1}\right) \rightarrow M\left(D\left(\left.\mathbf{u}\right|_{B_{k}}\right)^{-1}\right) \quad \text { in } L^{1}\left(V_{k}\right) \text { as } j \rightarrow \infty .
\end{aligned}
$$

Using, in addition, (7.29), the lower semicontinuity of polyconvex functionals (see, e.g., [8, Th. 5.4]) yields

$$
\begin{aligned}
\int_{V_{k}} \hat{W}\left(D\left(\left.\mathbf{u}\right|_{B_{k}}\right)^{-1}(\mathbf{y}), \mathbf{w}(\mathbf{y})\right) \mathrm{d} \mathbf{y} & \leq \liminf _{j \rightarrow \infty} \int_{V_{k}} \hat{W}\left(D\left(\left.\mathbf{u}_{j}\right|_{B_{k}}\right)^{-1}(\mathbf{y}), \mathbf{w}_{j}(\mathbf{y})\right) \mathrm{d} \mathbf{y} \\
& \leq \liminf _{j \rightarrow \infty} \int_{\operatorname{im}_{\mathrm{T}}\left(\mathbf{u}_{j}, B_{k}\right)} \hat{W}\left(D\left(\left.\mathbf{u}_{j}\right|_{B_{k}}\right)^{-1}(\mathbf{y}), \mathbf{w}_{j}(\mathbf{y})\right) \mathrm{d} \mathbf{y} .
\end{aligned}
$$

As $V_{k}$ is arbitrary in $\operatorname{im}_{\mathrm{T}}\left(\mathbf{u}, B_{k}\right)$, we obtain, by monotone convergence,

$$
\int_{\operatorname{im}_{\mathrm{T}}\left(\mathbf{u}, B_{k}\right)} \hat{W}\left(D\left(\left.\mathbf{u}\right|_{B_{k}}\right)^{-1}(\mathbf{y}), \mathbf{w}(\mathbf{y})\right) \mathrm{d} \mathbf{y} \leq \liminf _{j \rightarrow \infty} \int_{\operatorname{im}_{\mathrm{T}}\left(\mathbf{u}_{j}, B_{k}\right)} \hat{W}\left(D\left(\left.\mathbf{u}_{j}\right|_{B_{k}}\right)^{-1}(\mathbf{y}), \mathbf{w}_{j}(\mathbf{y})\right) \mathrm{d} \mathbf{y},
$$

which, thanks to (7.34)-(7.35), yields

$$
\int_{B_{k}} W_{0}(D \mathbf{u}(\mathbf{x}), \mathbf{w}(\mathbf{u}(\mathbf{x}))) \mathrm{d} \mathbf{x} \leq \liminf _{j \rightarrow \infty} \int_{B_{k}} W_{0}\left(D \mathbf{u}_{j}(\mathbf{x}), \mathbf{w}_{j}(\mathbf{u}(\mathbf{x}))\right) \mathrm{d} \mathbf{x} .
$$

Summing in $k \in \mathbb{N}$ the above inequality, we obtain (7.32).

Remark 7.9. The proof of Proposition 7.8 can easily be adapted to cover the incompressible regime: this corresponds to a choice of $W_{0}$ for which $W_{0}(\mathbf{F}, \mathbf{m})<\infty$ if and only if $\operatorname{det} \mathbf{F}=1$. For the proof in this case, one just has to realize that the lower semicontinuity theorem [8, Th. 5.4] remains valid, and that the function $\hat{W}(\cdot, \mathbf{m})$ of $(7.33)$ is polyconvex if and only if so is $W(\cdot, \mathbf{m})$. Therefore, inequalities (7.37) still hold in this case. Likewise, the proof can also be extended to cover an inhomogeneous stored-energy function $W_{0}$.

Remark 7.10. Conditions (7.30)-(7.31) ensure the local equiintegrability of $\left\{\operatorname{det} D\left(\left.\mathbf{u}_{j}\right|_{B_{k}}\right)^{-1}\right\}_{j \in \mathbb{N}}$. In Proposition 7.8 we had to assume $\mathbf{u} \in \mathcal{A}_{p}$ since that inclusion does not follow from Proposition 6.1 unless the equiintegrability of $\left\{\operatorname{det} D \mathbf{u}_{j}\right\}_{j \in \mathbb{N}}$ is guaranteed. In other words, the equiintegrability of the Jacobians of the local inverses is insufficient to yield the necessary compactness in $\mathcal{A}_{p}$. In fact, in the example of Ponomarev [67] mentioned in Remark 6.2 we have that $\sup _{j \in \mathbb{N}} \int_{\Omega} h\left(\operatorname{det} D \mathbf{u}_{j}\right)<\infty$ for a large class of functions $h$ satisfying $\lim _{t \searrow 0} h(t)=0$ and $\lim \sup _{t \rightarrow \infty} h(t) / t<\infty$; in particular, this is true if $h(t) \leq C\left(t^{-\alpha}+t+1\right)$ for all $t \in(0, \infty)$, with $\alpha<\frac{2}{\log _{3} 2}$, for the parameters fixed in [61, Sect. 11]. That sequence of homeomorphisms $\left\{\mathbf{u}_{j}\right\}_{j \in \mathbb{N}}$ satisfies that $\left\{\operatorname{det} D \mathbf{u}_{j}^{-1}\right\}_{j \in \mathbb{N}}$ is equiintegrable but $\left\{\operatorname{det} D \mathbf{u}_{j}\right\}_{j \in \mathbb{N}}$ is not. In practice, as we will see in Section 8, the existence of minimizers require the equiintegrability of the sequence of Jacobians of both the deformations and of their local inverses. This will be achieved by a suitable coercivity inequality of the energy, namely (8.2)-(8.3) below.

\section{Applications}

In this last section we apply the lower semicontinuity results of Section 7 to a series of models related to nonlinear elasticity and involving, in addition, energies defined in the deformed configuration. In those 
models, functionals of the form (7.1) will appear together with an additional term of the type (7.28), which provides the required compactness for $\mathbf{u}$ in order to have existence of minima. In general, the techniques developed in this paper are useful to deal with variational models that involve the deformed configuration (see Proposition 7.1) $(D \mathbf{u})^{-1}$ (see Proposition 7.3), the incompressibility constraint (see Proposition 7.6) and compositions of the form $\mathbf{w} \circ \mathbf{u}$ (see Proposition 7.8).

We have chosen three variational models that exemplify the results of Section 7 to prove existence of minimizers: a Frank energy model for nematic elastomers (Subsection 8.1), a Landau-de Gennes energy model for nematic elastomers (Subsection 8.2) and a model in magnetoelasticity (Subsection 8.3).

\subsection{A Frank energy model for nematic elastomers}

Liquid crystal elastomers are materials constituted by a network of cross-linked polymer chains. They combine properties of liquid crystals and rubber-like solids. In their inner structure, elongated rigid monomer units, called mesogens, are incorporated or attached sideways to the polymer chain. In the nematic phase, the mesogens self-align leading to an orientational order (but not to a positional one). When the orientational order is constrained to be uniaxial and the degree of order is fixed, it can be described by a director field n. The coupling between the nematic orientational order and rubber elasticity is the origin of a strong anisotropic behaviour: the alignment of nematic mesogens in a neighbourhood of a point $\mathbf{y}$ in the deformed configuration of the sample along an average direction $\mathbf{n}(\mathbf{y}) \in \mathbb{S}^{n-1}$ induces a spontaneous distortion given by

$$
\mathbf{V}_{\mathbf{n}}:=\alpha \mathbf{n} \otimes \mathbf{n}+\alpha^{\frac{1}{1-n}}(\mathbf{I}-\mathbf{n} \otimes \mathbf{n}) .
$$

This tensor, which is volume-preserving, i.e.,

$$
\operatorname{det} \mathbf{V}_{\mathbf{n}}=1
$$

represents a uniaxial stretch along the direction $\mathbf{n}$ of amplitude $\alpha>0$. The material parameter $\alpha$ describes the amount of the local distorsion. For $\alpha>1$ (prolate case) there is a spontaneous elongation along the director $\mathbf{n}$ and a contraction transverse to it. It is important to remark that, while in the small deformation regime, the director field $\mathbf{n}$ can be defined in the reference configuration, when large deformations are in order, it has to be evaluated in the deformed configuration.

We adopt the model described by Barchiesi \& DeSimone [11] (see also [1, 26]) and generalize it to the compressible case. Let $\Omega$ be a Lipschitz domain of $\mathbb{R}^{n}$, representing the reference configuration of the sample, $\Gamma$ an $(n-1)$-rectifiable subset of $\partial \Omega$ with $\mathcal{H}^{n-1}(\Gamma)>0$, and $\mathbf{u}_{0}: \Gamma \rightarrow \mathbb{R}^{n}$ a given function. As in the whole paper, $p>n-1$. The admissible set $\mathcal{B}$ for our problem is the set of pairs $(\mathbf{u}, \mathbf{n})$ where $\mathbf{u} \in \mathcal{A}_{p},\left.\mathbf{u}\right|_{\Gamma}=\mathbf{u}_{0}$ in the sense of traces and $\mathbf{n} \in W^{1,2}\left(\operatorname{im}_{\mathrm{T}}(\mathbf{u}, \Omega), \mathbb{S}^{n-1}\right)$. From the physical point of view, $\mathbf{u}$ represents an elastic deformation of the sample with a given boundary condition $\mathbf{u}_{0}$, while $\mathbf{n}$ is the nematic director field evaluated in the deformed configuration of the sample with respect to $\mathbf{u}$.

Let $W: \mathbb{R}_{+}^{n \times n} \rightarrow[0, \infty)$ be a polyconvex function. We assume that

$$
W(\mathbf{F}) \geq c|\mathbf{F}|^{p}+h(\operatorname{det} \mathbf{F}), \quad \mathbf{F} \in \mathbb{R}_{+}^{n \times n},
$$

for a constant $c>0$ and a Borel function $h:(0, \infty) \rightarrow[0, \infty)$ such that

$$
\lim _{t \searrow 0} h(t)=\lim _{t \rightarrow \infty} \frac{h(t)}{t}=\infty .
$$

The energy functional $I: \mathcal{B} \rightarrow[0, \infty]$ that describes the nematic elastomer is the sum of two contributions,

$$
I:=I_{\text {nem }}+I_{\text {mec }}, \quad \text { where } I_{\text {nem }}: \mathcal{B} \rightarrow[0, \infty) \text { and } I_{\text {mec }}: \mathcal{B} \rightarrow[0, \infty] .
$$

We call them the nematic and the mechanical term, respectively. The first one, defined by

$$
I_{\mathrm{nem}}(\mathbf{u}, \mathbf{n}):=\int_{\operatorname{im}_{\mathrm{T}}(\mathbf{u}, \Omega)}|D \mathbf{n}(\mathbf{y})|^{2} \mathrm{~d} \mathbf{y}
$$


is a simplified version of the Frank energy (see [19]). It penalizes the spatial non-uniformity of directors, without distinguishing the different types of distortion (splay, twist and bend). The second term captures, similarly to that developed by Warner \& Terentjev [74], the elasticity associated with the polymer chains of the nematic elastomer, taking into account the local anisotropy due to the director. It is defined as

$$
I_{\text {mec }}(\mathbf{u}, \mathbf{n}):=\int_{\Omega} W_{\text {mec }}(D \mathbf{u}(\mathbf{x}), \mathbf{n}(\mathbf{u}(\mathbf{x}))) \mathrm{d} \mathbf{x} .
$$

The mechanical response $W_{\text {mec }}: \mathbb{R}_{+}^{n \times n} \times \mathbb{S}^{n-1} \rightarrow[0, \infty)$ describes the coupling between the deformation and the director field through the formula

$$
W_{\mathrm{mec}}(\mathbf{F}, \mathbf{n}):=W\left(\mathbf{V}_{\mathbf{n}}^{-1} \mathbf{F}\right) .
$$

Note that the gradient operator in (8.5) is meant with respect to the current spatial variable $\mathbf{y}$, while the gradient in (8.6) is with respect to the material coordinate $\mathbf{x}$. We show that $W_{\text {mec }}$ is polyconvex.

Lemma 8.1. Let $W: \mathbb{R}_{+}^{n \times n} \rightarrow[0, \infty)$ be a polyconvex function and let $W_{\mathrm{mec}}: \mathbb{R}_{+}^{n \times n} \times \mathbb{S}^{n-1} \rightarrow[0, \infty)$ be defined as (8.7). Then $W_{\text {mec }}(\cdot, \mathbf{n})$ is polyconvex for each $\mathbf{n} \in \mathbb{S}^{n-1}$.

Proof. Let $\tau$ be the number of minors of an $\mathbb{R}^{n \times n}$ matrix, and let $\mathbf{M}: \mathbb{R}_{+}^{n \times n} \rightarrow \mathbb{R}^{\tau}$ be the function that assigns to each matrix in $\mathbb{R}_{+}^{n \times n}$ the collection of its minors, in a given order. By assumption, there exists a convex function $\Phi: \mathbb{R}^{\tau} \rightarrow[0, \infty]$ such that $W(\mathbf{F})=\Phi(\mathbf{M}(\mathbf{F}))$ for all $\mathbf{F} \in \mathbb{R}_{+}^{n \times n}$. Thus, for each $\mathbf{F} \in \mathbb{R}_{+}^{n \times n}$ and $\mathbf{n} \in \mathbb{S}^{n-1}$ we have $W_{\text {mec }}(\mathbf{F}, \mathbf{n})=\Phi\left(\mathbf{M}\left(\mathbf{V}_{\mathbf{n}}^{-1} \mathbf{F}\right)\right)$. Now, as a consequence of the Cauchy-Binet formula for the minors of a product of matrices (see, e.g., [37, Sect. 1.2]), there exists a bilinear map $\mathbf{C}: \mathbb{R}^{\tau} \times \mathbb{R}^{\tau} \rightarrow \mathbb{R}^{\tau}$ such that

$$
\mathbf{M}(\mathbf{A} \mathbf{B})=\mathbf{C}(\mathbf{M}(\mathbf{A}), \mathbf{M}(\mathbf{B})), \quad \mathbf{A}, \mathbf{B} \in \mathbb{R}^{n \times n} .
$$

Incidentally, all entries of the third-order tensor $\mathbf{C}$ are in $\{0,1\}$, but this is not important in the proof. Therefore,

$$
W_{\text {mec }}(\mathbf{F}, \mathbf{n})=\Phi\left(\mathbf{C}\left(\mathbf{M}\left(\mathbf{V}_{\mathbf{n}}^{-1}\right), \mathbf{M}(\mathbf{F})\right)\right), \quad \mathbf{F} \in \mathbb{R}_{+}^{n \times n}, \quad \mathbf{n} \in \mathbb{S}^{n-1},
$$

and this expression reveals that $W_{\text {mec }}(\cdot, \mathbf{n})$ is polyconvex for each $\mathbf{n} \in \mathbb{S}^{n-1}$, since the function $\Phi\left(\mathbf{C}\left(\mathbf{M}\left(\mathbf{V}_{\mathbf{n}}^{-1}\right), \cdot\right)\right)$ is easily seen to be convex in $\mathbb{R}^{\tau}$.

Proposition 7.1 covers the functional $I_{\text {nem }}$, while Proposition 7.8 covers $I_{\mathrm{mec}}$. The main result of this section shows the existence of minimizers of $I$ in $\mathcal{B}$. The differences with respect to [11, Th. 1] are that we weaken the coercivity condition from $p=n$ to $p>n-1$, and that we treat both compressible and incompressible cases.

Theorem 8.2. Let $\Omega$ be a Lipschitz domain of $\mathbb{R}^{n}, \Gamma$ an $(n-1)$-rectifiable subset of $\partial \Omega$ with $\mathcal{H}^{n-1}(\Gamma)>0$, and $\mathbf{u}_{0}: \Gamma \rightarrow \mathbb{R}^{n}$. Let $p>n-1$, define $\mathcal{B}$ as the set of $(\mathbf{u}, \mathbf{n})$ where $\mathbf{u} \in \mathcal{A}_{p},\left.\mathbf{u}\right|_{\Gamma}=\mathbf{u}_{0}$ and $\mathbf{n} \in$ $W^{1,2}\left(\mathrm{im}_{\mathrm{T}}(\mathbf{u}, \Omega), \mathbb{S}^{n-1}\right)$. Let $W: \mathbb{R}_{+}^{n \times n} \rightarrow[0, \infty)$ be a polyconvex function such that equations (8.2)-(8.3) hold for a constant $c>0$ and a Borel function $h:(0, \infty) \rightarrow[0, \infty)$. Define $I$ as in (8.4)-(8.7).

If $\mathcal{B} \neq \varnothing$ and $I$ is not identically infinity in $\mathcal{B}$, then $I$ attains its minimum in $\mathcal{B}$.

Proof. Let $\left\{\left(\mathbf{u}_{j}, \mathbf{n}_{j}\right)\right\}_{j \in \mathbb{N}}$ be a minimizing sequence of $I$ in $\mathcal{B}$. As

$$
\sup _{j \in \mathbb{N}}\left\|\mathbf{V}_{\mathbf{n}_{j}}\right\|_{L^{\infty}\left(\mathrm{im}_{\mathrm{T}}\left(\mathbf{u}_{j}, \Omega\right)\right)}<\infty,
$$

assumptions (8.2)-(8.3), equality (8.1) and de la Vallée-Poussin's criterion imply that $\left\{D \mathbf{u}_{j}\right\}_{j \in \mathbb{N}}$ is bounded in $L^{p}\left(\Omega, \mathbb{R}^{n \times n}\right)$ and $\left\{\operatorname{det} D \mathbf{u}_{j}\right\}_{j \in \mathbb{N}}$ is equiintegrable. By the Poincaré inequality and the boundary condition, $\left\{\mathbf{u}_{j}\right\}_{j \in \mathbb{N}}$ is bounded in $W^{1, p}\left(\Omega, \mathbb{R}^{n}\right)$. By Proposition 6.1, there exists $\mathbf{u} \in W^{1, p}\left(\Omega, \mathbb{R}^{n}\right)$ such that $\operatorname{det} D \mathbf{u} \geq 0$ a.e., $\mathcal{E}(\mathbf{u})=0$ and, for a subsequence (not relabelled),

$$
\mathbf{u}_{j} \rightarrow \mathbf{u} \text { in } W^{1, p}\left(\Omega, \mathbb{R}^{n}\right) \quad \text { and } \quad \operatorname{det} D \mathbf{u}_{j} \rightarrow \operatorname{det} D \mathbf{u} \text { in } L^{1}(\Omega) \quad \text { as } j \rightarrow \infty .
$$


If $\operatorname{det} D \mathbf{u}$ were zero in a set $A$ of positive measure, then we would have (for a subsequence) $\operatorname{det} D \mathbf{u}_{j} \rightarrow 0$ a.e. in $A$; by (8.3), we would obtain $h\left(\operatorname{det} D \mathbf{u}_{j}\right) \rightarrow \infty$ a.e. in $A$, as $j \rightarrow \infty$, so, by Fatou's lemma and (8.2), we would get $I_{\text {mec }}\left(\mathbf{u}_{j}, \mathbf{n}_{j}\right) \rightarrow \infty$ as $j \rightarrow \infty$, which is a contradiction. Therefore, $\operatorname{det} D \mathbf{u}>0$ a.e., so $\mathbf{u} \in \mathcal{A}_{p}$. Moreover, the boundary condition is also preserved under the limit, so $\left.\mathbf{u}\right|_{\Gamma}=\mathbf{u}_{0}$ in the sense of traces. From the boundedness of $\left\{\operatorname{det} D \mathbf{u}_{j}\right\}_{j \in \mathbb{N}}$ in $L^{1}(\Omega)$ it follows that of $\left\{\left\|\mathbf{n}_{j}\right\|_{L^{2}\left(\mathrm{im}_{\mathrm{T}}\left(\mathbf{u}_{j}, \Omega\right), \mathbb{R}^{n}\right)}\right\}_{j \in \mathbb{N}}$, because by Proposition 2.7 and Lemma $5.18 c$ ) one has for each $j \in \mathbb{N}$,

$$
\int_{\operatorname{im}_{\mathrm{T}}\left(\mathbf{u}_{j}, \Omega\right)}\left|\mathbf{n}_{j}\right|^{2} \mathrm{~d} \mathbf{y}=\mathcal{L}^{n}\left(\operatorname{im}_{\mathrm{T}}\left(\mathbf{u}_{j}, \Omega\right)\right)=\mathcal{L}^{n}\left(\operatorname{im}_{\mathrm{G}}\left(\mathbf{u}_{j}, \Omega\right)\right) \leq \int_{\Omega} \operatorname{det} D \mathbf{u}_{j}(\mathbf{x}) \mathrm{d} \mathbf{x} .
$$

Since $\left\{\left\|\mathbf{n}_{j}\right\|_{W^{1,2}\left(\operatorname{im}_{\mathrm{T}}\left(\mathbf{u}_{j}, \Omega\right), \mathbb{R}^{n}\right)}\right\}_{j \in \mathbb{N}}$ is bounded, by Proposition 7.1 , there exist $\mathbf{n} \in W^{1,2}\left(\operatorname{im}_{\mathrm{T}}(\mathbf{u}, \Omega), \mathbb{S}^{n-1}\right)$ and a subsequence such that $(\mathbf{u}, \mathbf{n}) \in \mathcal{B}$,

$$
\begin{aligned}
& \chi_{\operatorname{im}_{\mathrm{T}}\left(\mathbf{u}_{j}, \Omega\right)} \mathbf{n}_{j} \rightarrow \chi_{\operatorname{im}_{\mathrm{T}}(\mathbf{u}, \Omega)} \mathbf{n} \text { a.e. and } \\
& \chi_{\operatorname{im}_{\mathrm{T}}\left(\mathbf{u}_{j}, \Omega\right)} D \mathbf{n}_{j} \rightarrow \chi_{\operatorname{im}_{\mathrm{T}}(\mathbf{u}, \Omega)} D \mathbf{n} \quad \text { in } L^{2}\left(\mathbb{R}^{n}, \mathbb{R}^{n \times n}\right) \quad \text { as } j \rightarrow \infty
\end{aligned}
$$

and

$$
I_{\mathrm{nem}}(\mathbf{u}, \mathbf{n})=\int_{\operatorname{im}_{\mathrm{T}}(\mathbf{u}, \Omega)}|D \mathbf{n}(\mathbf{y})|^{2} \mathrm{~d} \mathbf{y} \leq \liminf _{j \rightarrow \infty} \int_{\operatorname{im}_{\mathrm{T}}\left(\mathbf{u}_{j}, \Omega\right)}\left|D \mathbf{n}_{j}(\mathbf{y})\right|^{2} \mathrm{~d} \mathbf{y}=\liminf _{j \rightarrow \infty} I_{\mathrm{nem}}\left(\mathbf{u}_{j}, \mathbf{n}_{j}\right) .
$$

Using Proposition 7.8 and Lemma 8.1 we obtain that

$$
\int_{\Omega} W_{\mathrm{mec}}(D \mathbf{u}(\mathbf{x}), \mathbf{n}(\mathbf{u}(\mathbf{x}))) \mathrm{d} \mathbf{x} \leq \liminf _{j \rightarrow \infty} \int_{\Omega} W_{\operatorname{mec}}\left(D \mathbf{u}_{j}(\mathbf{x}), \mathbf{n}_{j}\left(\mathbf{u}_{j}(\mathbf{x})\right)\right) \mathrm{d} \mathbf{x},
$$

which, with (8.8), shows that $(\mathbf{u}, \mathbf{n})$ is a minimizer of $I$ in $\mathcal{B}$.

Remark 8.3. In Theorem 8.2, it is possible to replace the admissible set $\mathcal{B}$ with the more physically relevant set

$$
\mathcal{B}_{\text {in }}:=\{(\mathbf{u}, \mathbf{n}) \in \mathcal{B}: \mathbf{u} \text { is one-to-one a.e. in } \Omega\} .
$$

Indeed, as proved in [42, Th. 1], the global invertibility condition is preserved under the weak limit. Therefore, the restriction of $I$ to $\mathcal{B}_{\text {in }}$ still has a minimum.

Remark 8.4. Recalling Remark 7.9, we can see that the proof of Theorem 8.2 can easily be adapted to cover the incompressible regime: this corresponds to the choice of $h$ to be $h(1)=0$ and $h(t)=\infty$ for $t \neq 1$. Likewise, the proof can also be extended to cover an inhomogeneous stored-energy function $W$, with its corresponding inhomogeneous mechanical response $W_{\text {mec }}$.

\subsection{A Landau-de Gennes energy model for nematic elastomers}

Our method can also be used in the more elaborated theory of Landau-de Gennes for liquid crystal elastomers. While the Frank theory only considers a uniaxial nematic order, which is described through the director field $\mathbf{n}$, the Landau-de Gennes theory allows more degrees of freedom, and uses a function $\mathbf{Q}$, referred to as the nematic order tensor, taking values in the space of $n \times n$ symmetric and traceless matrices. This tensor $\mathbf{Q}$ is a normalized second moment of the probability distribution describing the orientation of the molecules. Instead of $\mathbf{V}_{\mathbf{n}}$, the local distorsion of the nematic is encoded in the step-length tensor $\mathbf{L}_{\mathbf{Q}}$ to be defined below.

We adopt the model described by Calderer, Garavito Garzón \& Yan [14], which we start describing by introducing some notation. We let $n=3$, which is the physically relevant case; we also note that the constants involved below are dimension-dependent. We consider the following admisible sets of matrices for the nematic order tensor:

$$
\begin{aligned}
& \mathbb{Q}:=\left\{\mathbf{Q} \in \mathbb{R}^{n \times n}: \mathbf{Q}=\mathbf{Q}^{T}, \operatorname{tr} \mathbf{Q}=0,-\frac{1}{3} \leq \lambda_{\min }(\mathbf{Q}) \leq \lambda_{\max }(\mathbf{Q}) \leq \frac{2}{3}\right\}, \\
& \tilde{\mathbb{Q}}:=\left\{\mathbf{Q} \in \mathbb{Q}: \lambda_{\min }(\mathbf{Q})>-\frac{1}{3}\right\},
\end{aligned}
$$


where $\lambda_{\min }(\mathbf{Q})$ and $\lambda_{\max }(\mathbf{Q})$ denote the minimum and maximum eigenvalues of $\mathbf{Q}$, respectively. Given $\mathbf{Q} \in \mathbb{Q}$ we define the step-length tensor

$$
\mathbf{L}_{\mathbf{Q}}:=\alpha\left(\mathbf{Q}+\frac{1}{3} \mathbf{I}\right)
$$

where $\alpha>0$ is a fixed parameter depending on the material. As $\mathbf{Q}$ is symmetric, there exists an orthogonal matrix $\mathbf{P}$ such that $\mathbf{P}^{-1} \mathbf{Q P}$ is diagonal. We have $\mathbf{P}^{-1} \mathbf{L}_{\mathbf{Q}} \mathbf{P}=\alpha\left(\mathbf{P}^{-1} \mathbf{Q P}+\frac{1}{3} \mathbf{I}\right)$ and, consequently,

$$
\lambda_{\min }\left(\mathbf{L}_{\mathbf{Q}}\right)=\alpha\left(\lambda_{\min }(\mathbf{Q})+\frac{1}{3}\right) \geq 0 \quad \text { and } \quad \lambda_{\max }\left(\mathbf{L}_{\mathbf{Q}}\right)=\alpha\left(\lambda_{\max }(\mathbf{Q})+\frac{1}{3}\right) .
$$

In particular,

$$
\operatorname{det} \mathbf{L}_{\mathbf{Q}}=0 \text { if and only if } \lambda_{\min }(\mathbf{Q})=-\frac{1}{3} .
$$

Since $\mathbf{L}_{\mathbf{Q}}$ is symmetric and positive semidefinite, it has a square $\operatorname{root} \mathbf{L}_{\mathbf{Q}}^{\frac{1}{2}}$ and its eigenvalues are the square roots of those of $\mathbf{L}_{\mathbf{Q}}$. Finally, note that

$$
|\mathbf{Q}| \leq\left(\frac{2}{3}\right)^{\frac{1}{2}}, \quad\left|\mathbf{L}_{\mathbf{Q}}^{\frac{1}{2}}\right| \leq \sqrt{\alpha} \quad \text { and } \quad 0 \leq \operatorname{det} \mathbf{L}_{\mathbf{Q}} \leq\left(\frac{\alpha}{3}\right)^{3},
$$

as can be seen by expressing $\mathbf{Q}$, without loss of generality, as a diagonal matrix with entries $\lambda_{\min }(\mathbf{Q}), \lambda_{\max }(\mathbf{Q})$ and $-\lambda_{\min }(\mathbf{Q})-\lambda_{\max }(\mathbf{Q})$, and then using an elementary argument of maximization.

Similarly to the previous model, $\Omega$ is a Lipschitz domain of $\mathbb{R}^{n}, \Gamma$ an $(n-1)$-rectifiable subset of $\partial \Omega$ with $\mathcal{H}^{n-1}(\Gamma)>0, \mathbf{u}_{0}: \Gamma \rightarrow \mathbb{R}^{n}$ a given function, $p>n-1$ and $q>1$. Now, the admissible set $\mathcal{B}$ is the set of pairs $(\mathbf{u}, \mathbf{Q})$ such that $\mathbf{u} \in \mathcal{A}_{p},\left.\mathbf{u}\right|_{\Gamma}=\mathbf{u}_{0}$ in the sense of traces, and $\mathbf{Q} \in W^{1, q}\left(\operatorname{im}_{\mathrm{T}}(\mathbf{u}, \Omega), \tilde{\mathbb{Q}}\right)$. The tensor $\mathbf{Q}$ is a normalized second moment of a probability distribution function $\psi=\psi(\mathbf{n})$ that describes the probability of finding the nematic mesogens oriented in a direction $\mathbf{n} \in \mathbb{S}^{n-1}$. The choice of the target $\tilde{\mathbb{Q}}$ for $\mathbf{Q}$ is motivated in [55] (see also [9]) in terms of the mean-field Maier-Saupe theory. Note that the symbol $\mathbf{Q}$ is sometimes used for an element of $\tilde{\mathbb{Q}}$, but, more often, it will be used for a function in $W^{1, q}\left(\operatorname{im}_{\mathrm{T}}(\mathbf{u}, \Omega), \tilde{\mathbb{Q}}\right)$. Analogously, $\mathbf{L}_{\mathbf{Q}}$ sometimes denotes a matrix, but, more often, a function.

The free energy functional $I: \mathcal{B} \rightarrow[0, \infty]$ of a nematic elastomer is the sum of two terms:

$$
I:=I_{\mathrm{mec}}+I_{\mathrm{LG}}
$$

The elastic stored energy $I_{\text {mec }}$ is given by

$$
I_{\mathrm{mec}}(\mathbf{u}, \mathbf{Q}):=\int_{\Omega} W_{\mathrm{mec}}(D \mathbf{u}(\mathbf{x}), \mathbf{Q}(\mathbf{u}(\mathbf{x}))) \mathrm{d} \mathbf{x}
$$

where $W_{\text {mec }}: \mathbb{R}_{+}^{n \times n} \times \tilde{\mathbb{Q}} \rightarrow[0, \infty)$ is defined by

$$
W_{\text {mec }}(\mathbf{F}, \mathbf{Q}):=W\left(\mathbf{L}_{\mathbf{Q}}^{-\frac{1}{2}} \mathbf{F}\right)
$$

for a certain polyconvex function $W: \mathbb{R}_{+}^{n \times n} \rightarrow[0, \infty)$ satisfying the coercivity conditions (8.2)-(8.3) for a constant $c>0$ and a Borel function $h:(0, \infty) \rightarrow[0, \infty)$. The product $\mathbf{L}_{\mathbf{Q}}^{-\frac{1}{2}} \mathbf{F}$ represents the coupling between the deformation and the nematic tensor order. The Landau-de Gennes energy $I_{\mathrm{LG}}$ is given by

$$
I_{\mathrm{LG}}(\mathbf{u}, \mathbf{Q}):=\int_{\operatorname{im}_{\mathrm{T}}(\mathbf{u}, \Omega)} f(D \mathbf{Q}(\mathbf{y}), \mathbf{Q}(\mathbf{y})) \mathrm{d} \mathbf{y},
$$

for some lower semicontinuous function $f: \mathbb{A} \times \tilde{\mathbb{Q}} \rightarrow[0, \infty]$. We have denoted by $\mathbb{A}$ the subspace of thirdorder tensors $\mathbf{T}=\left(T_{k}^{i j}\right)_{i, j, k=1}^{n}$ such that $T_{k}^{i j}=T_{k}^{j i}$ for all $i, j, k \in\{1, \ldots, n\}$ and $\sum_{i=1}^{n} T_{k}^{i i}=0$ for all $k \in\{1, \ldots, n\}$. Of course, $D \mathbf{Q}(\mathbf{y}) \in \mathbb{A}$ for all $\mathbf{Q} \in W^{1, q}\left(\operatorname{im}_{\mathrm{T}}(\mathbf{u}, \Omega), \tilde{\mathbb{Q}}\right)$ and a.e. $\mathbf{y} \in \operatorname{im}_{\mathrm{T}}(\mathbf{u}, \Omega)$. We assume that $f(\cdot, \mathbf{Q})$ is convex for all $\mathbf{Q} \in \tilde{\mathbb{Q}}$ and that

$$
f(\mathbf{T}, \mathbf{Q}) \geq c|\mathbf{T}|^{q}+g\left(\operatorname{det} \mathbf{L}_{\mathbf{Q}}\right), \quad \mathbf{T} \in \mathbb{A}, \quad \mathbf{Q} \in \tilde{\mathbb{Q}}
$$


for a constant $c>0$ and a Borel function $g:(0, \infty) \rightarrow[0, \infty)$ such that

$$
\lim _{t \searrow 0} g(t)=\infty .
$$

Note that the gradient operator in (8.14) is meant with respect to the current spatial variable $\mathbf{y}$, while the gradient in (8.12) is with respect to the material coordinate $\mathbf{x}$.

By using the lower semicontinuity results of Propositions 7.1 and 7.8 , we can weaken the coercivity conditions imposed in [14] from $p>n$ to $p>n-1$, and from $q>\max \{3, p /(p-3)\}$ to $q>1$; in particular, $f$ is allowed to have a quadratic growth. In addition, we do not impose that $\operatorname{det} D \mathbf{u}$ is bounded away from zero.

The following result can be proved exactly as in Lemma 8.1.

Lemma 8.5. Let $W: \mathbb{R}_{+}^{n \times n} \rightarrow[0, \infty)$ be a polyconvex function and let $W_{\mathrm{mec}}: \mathbb{R}_{+}^{n \times n} \times \mathbb{S}^{n-1} \rightarrow[0, \infty)$ be defined as (8.13). Then $W_{\text {mec }}(\cdot, \mathbf{Q})$ is polyconvex for each $\mathbf{Q} \in \tilde{\mathbb{Q}}$.

The existence theorem is as follows.

Theorem 8.6. Let $n=3, \Omega$ be a Lipschitz domain of $\mathbb{R}^{n}, \Gamma$ an $(n-1)$-rectifiable subset of $\partial \Omega$ with $\mathcal{H}^{n-1}(\Gamma)>0$, and $\mathbf{u}_{0}: \Gamma \rightarrow \mathbb{R}^{n}$. Let $p>n-1$ and $q>1$, define $\mathcal{B}$ as the set of $(\mathbf{u}, \mathbf{Q})$ where $\mathbf{u} \in \mathcal{A}_{p}$, $\left.\mathbf{u}\right|_{\Gamma}=\mathbf{u}_{0}$ and $\mathbf{Q} \in W^{1, q}\left(\operatorname{im}_{\mathrm{T}}(\mathbf{u}, \Omega), \tilde{\mathbb{Q}}\right)$. Let $W: \mathbb{R}_{+}^{n \times n} \rightarrow[0, \infty)$ be a polyconvex function such that equations (8.2)-(8.3) hold for a constant $c>0$ and a Borel function $h:(0, \infty) \rightarrow[0, \infty)$. Let $f: \mathbb{A} \times \tilde{\mathbb{Q}} \rightarrow[0, \infty]$ be a lower semicontinuous function such that $f(\cdot, \mathbf{Q})$ is convex for all $\mathbf{Q} \in \tilde{\mathbb{Q}}$ and that equations (8.15)-(8.16) hold for a constant $c>0$ and a Borel function $g:(0, \infty) \rightarrow[0, \infty)$. Define $I$ as in (8.11)-(8.14).

If $\mathcal{B} \neq \varnothing$ and $I$ is not identically infinity in $\mathcal{B}$, then $I$ attains its minimum in $\mathcal{B}$.

Proof. The proof is similar to that of Theorem 8.2. Let $\left\{\left(\mathbf{u}_{j}, \mathbf{Q}_{j}\right)\right\}_{j \in \mathbb{N}}$ be a minimizing sequence of $I$ in $\mathcal{B}$. We write $\mathbf{L}_{j}:=\mathbf{L}_{\mathbf{Q}_{j}}$ and $\mathbf{G}_{j}:=\left(\mathbf{L}_{j}^{-\frac{1}{2}} \circ \mathbf{u}_{j}\right) D \mathbf{u}_{j}$, for each $j \in \mathbb{N}$. The following pointwise bounds follow from (8.10):

$$
\left|D \mathbf{u}_{j}\right| \leq\left|\mathbf{L}_{j}^{\frac{1}{2}} \circ \mathbf{u}_{j}\right|\left|\mathbf{G}_{j}\right| \leq \sqrt{\alpha}\left|\mathbf{G}_{j}\right| \quad \text { and } \operatorname{det} D \mathbf{u}_{j}=\operatorname{det}\left(\mathbf{L}_{j}^{\frac{1}{2}} \circ \mathbf{u}_{j}\right) \operatorname{det} \mathbf{G}_{j} \leq\left(\frac{\alpha}{3}\right)^{\frac{3}{2}} \operatorname{det} \mathbf{G}_{j} .
$$

Consequently, assumptions (8.2)-(8.3) and de la Vallée-Poussin's criterion ensure that $\left\{D \mathbf{u}_{j}\right\}_{j \in \mathbb{N}}$ is bounded in $L^{p}\left(\Omega, \mathbb{R}^{n \times n}\right)$ and $\left\{\operatorname{det} D \mathbf{u}_{j}\right\}_{j \in \mathbb{N}}$ is equiintegrable. As in Theorem 8.2, the Poincaré inequality, the boundary condition, Proposition 6.1 and the growth condition (8.3) imply that, for a subsequence, $\mathbf{u}_{j} \rightarrow \mathbf{u}$ in $W^{1, p}\left(\Omega, \mathbb{R}^{n}\right)$ as $j \rightarrow \infty$, for a certain $\mathbf{u} \in \mathcal{A}_{p}$ such that $\left.\mathbf{u}\right|_{\Gamma}=\mathbf{u}_{0}$.

Thanks to (8.10) and (8.15), we have

$$
\sup _{j \in \mathbb{N}}\left[\left\|\mathbf{Q}_{j}\right\|_{L^{\infty}\left(\operatorname{im}_{\mathrm{T}}\left(\mathbf{u}_{j}, \Omega\right), \mathbb{R}^{n \times n}\right)}+\left\|D \mathbf{Q}_{j}\right\|_{L^{q}\left(\operatorname{im}_{\mathrm{T}}\left(\mathbf{u}_{j}, \Omega\right), \mathbb{A}\right)}\right]<\infty .
$$

Since, in addition, by Proposition 2.7 and Lemma $5.18 c$ ) one has for each $j \in \mathbb{N}$,

$$
\mathcal{L}^{n}\left(\operatorname{im}_{\mathrm{T}}\left(\mathbf{u}_{j}, \Omega\right)\right)=\mathcal{L}^{n}\left(\operatorname{im}_{\mathrm{G}}\left(\mathbf{u}_{j}, \Omega\right)\right) \leq \int_{\Omega} \operatorname{det} D \mathbf{u}_{j}(\mathbf{x}) \mathrm{d} \mathbf{x},
$$

we obtain that

$$
\sup _{j \in \mathbb{N}}\left\|\mathbf{Q}_{j}\right\|_{W^{1, q}\left(\operatorname{im}_{\mathrm{T}}\left(\mathbf{u}_{j}, \Omega\right), \mathbb{R}^{n \times n}\right)}<\infty .
$$

Therefore, due to Proposition 7.1, there exists $\mathbf{Q} \in W^{1, q}\left(\operatorname{im}_{\mathrm{T}}(\mathbf{u}, \Omega), \mathbb{Q}\right)$ such that, for a subsequence,

$$
\begin{aligned}
& \chi_{\mathrm{im}_{\mathrm{T}}\left(\mathbf{u}_{j}, \Omega\right)} \mathbf{Q}_{j} \rightarrow \chi_{\mathrm{im}_{\mathrm{T}}(\mathbf{u}, \Omega)} \mathbf{Q} \text { a.e. and in } L^{q}\left(\mathbb{R}^{n}, \mathbb{R}^{n \times n}\right) \\
& \chi_{\mathrm{im}_{\mathrm{T}}\left(\mathbf{u}_{j}, \Omega\right)} D \mathbf{Q}_{j} \rightarrow \chi_{\operatorname{im}_{\mathrm{T}}(\mathbf{u}, \Omega)} D \mathbf{Q} \text { in } L^{q}\left(\mathbb{R}^{n}, \mathbb{R}^{n \times n \times n}\right) \text { as } j \rightarrow \infty .
\end{aligned}
$$


We now proceed as in the proof of Proposition 7.1. Let $\left\{V_{k}\right\}_{k \in \mathbb{N}}$ be the family of Lemma $5.18 d$ ). For each $k \in \mathbb{N}$, let $B_{k} \in \mathcal{U}_{\mathbf{u}}^{N}$ be such that $V_{k} \subset \subset \operatorname{im}_{\mathrm{T}}\left(\mathbf{u}, B_{k}\right)$. Since, by Theorem $\left.6.3 a\right), V_{k} \subset \operatorname{im}_{\mathrm{T}}\left(\mathbf{u}_{j}, \Omega\right)$ for a subsequence, convergences (8.17) imply $\mathbf{Q}_{j} \rightarrow \mathbf{Q}$ in $L^{q}\left(V_{k}, \mathbb{Q}\right)$ and $\mathbf{Q}_{j} \rightarrow \mathbf{Q}$ in $W^{1, q}\left(V_{k}, \mathbb{Q}\right)$ as $j \rightarrow \infty$.

Let $\bar{f}: \mathbb{A} \times \mathbb{Q} \rightarrow[0, \infty]$ be the extension by $\infty$ of $f$ and note that, thanks to (8.15)-(8.16), $\bar{f}$ is lower semicontinuous and $\bar{f}\left(\cdot, \mathbf{Q}_{0}\right)$ is convex for each $\mathbf{Q}_{0} \in \mathbb{Q}$. Then, a standard lower semicontinuity result yields (see, e.g., [33, Th. 7.5])

$$
\int_{V_{k}} \bar{f}(D \mathbf{Q}(\mathbf{y}), \mathbf{Q}(\mathbf{y})) \mathrm{d} \mathbf{y} \leq \liminf _{j \rightarrow \infty} \int_{V_{k}} \bar{f}\left(D \mathbf{Q}_{j}(\mathbf{y}), \mathbf{Q}_{j}(\mathbf{y})\right) \mathrm{d} \mathbf{y}=\liminf _{j \rightarrow \infty} \int_{\operatorname{im}_{\mathrm{T}}\left(\mathbf{u}_{j}, \Omega\right) \cap V_{k}} \bar{f}\left(D \mathbf{Q}_{j}(\mathbf{y}), \mathbf{Q}_{j}(\mathbf{y})\right) \mathrm{d} \mathbf{y} .
$$

Summing in $k \in \mathbb{N}$ the above inequalities, we obtain

$$
\int_{\operatorname{im}_{\mathrm{T}}(\mathbf{u}, \Omega)} \bar{f}(D \mathbf{Q}(\mathbf{y}), \mathbf{Q}(\mathbf{y})) \mathrm{d} \mathbf{y} \leq \liminf _{j \rightarrow \infty} \int_{\operatorname{im}_{\mathrm{T}}\left(\mathbf{u}_{j}, \Omega\right)} \bar{f}\left(D \mathbf{Q}_{j}(\mathbf{y}), \mathbf{Q}_{j}(\mathbf{y})\right) \mathrm{d} \mathbf{y}<\infty .
$$

In particular, by (8.15)-(8.16) we have that $\operatorname{det} \mathbf{L}_{\mathbf{Q}}(\mathbf{y})>0$ for a.e. $\mathbf{y} \in \operatorname{im}_{\mathrm{T}}(\mathbf{u}, \Omega)$, so, by equivalence (8.9) we obtain that $\mathbf{Q} \in W^{1, q}\left(\operatorname{im}_{\mathrm{T}}(\mathbf{u}, \Omega), \tilde{\mathbb{Q}}\right),(\mathbf{u}, \mathbf{Q}) \in \mathcal{B}$ and

$$
I_{\mathrm{LG}}(\mathbf{u}, \mathbf{Q}) \leq \liminf _{j \rightarrow \infty} I_{\mathrm{LG}}\left(\mathbf{u}_{j}, \mathbf{Q}_{j}\right) .
$$

By (8.17) and Theorem $6.3 d$ ) we also obtain $\mathbf{Q}_{j} \rightarrow \mathbf{Q}$ a.e. Thus, Lemma 8.5 and Proposition 7.8 yield

$$
I_{\text {mec }}(\mathbf{u}, \mathbf{Q}) \leq \liminf _{j \rightarrow \infty} I_{\text {mec }}\left(\mathbf{u}_{j}, \mathbf{Q}_{j}\right)
$$

which, together with (8.18), shows that $(\mathbf{u}, \mathbf{Q})$ is a minimizer of $I$ in $\mathcal{B}$.

Remark 8.7. Recalling Remarks 8.3 and 8.4, we can see that the proof of Theorem 8.6 can easily be adapted to cover the case of deformations that are one-to-one a.e. in $\Omega$, as well as the incompressible regime and an inhomogeneous stored energy $W$.

\subsection{Magnetoelasticity}

The mechanical behaviour of certain materials, notably ferromagnetic materials, is affected by the presence of a magnetic field. This field causes the rotations of small magnetic domains of the body from their original random orientation and induces a spontaneous deformation of the material.

For this analysis, we adopt the magnetostriction model of Rybka \& Luskin [69] and Kružík, Stefanelli \& Zeman [53], following earlier models by James \& Kinderlehrer [49, 50]. We refer to [23, 24, 25] for further discussions on the subject. The mathematical setting is similar to the Frank model for nematic elastomers described in Subsection 8.1: $\Omega$ is a Lipschitz domain of $\mathbb{R}^{n}$ representing the body in its reference configuration, $\Gamma$ an $(n-1)$-rectifiable subset of $\partial \Omega$ with $\mathcal{H}^{n-1}(\Gamma)>0$, and $\mathbf{u}_{0}: \Gamma \rightarrow \mathbb{R}^{n}$ is a given function prescribing the Dirichlet condition. The deformation of the specimen is $\mathbf{u}: \Omega \rightarrow \mathbb{R}^{n}$, while the magnetization $\mathbf{m}$ is defined in the deformed configuration, which in this analysis is represented by $\operatorname{im}_{\mathrm{T}}(\mathbf{u}, \Omega)$. The admissible set $\mathcal{B}$ is the set of pairs $(\mathbf{u}, \mathbf{m})$ where $\mathbf{u} \in \mathcal{A}_{p},\left.\mathbf{u}\right|_{\Gamma}=\mathbf{u}_{0}$ in the sense of traces and $\mathbf{m} \in W^{1,2}\left(\operatorname{im}_{\mathrm{T}}(\mathbf{u}, \Omega), \mathbb{S}^{n-1}\right)$; with respect to the Frank model for nematic elastomers, we have just renamed the function $\mathbf{n}$ as $\mathbf{m}$.

The energy functional $I: \mathcal{B} \rightarrow[0, \infty]$,

$$
I:=I_{\mathrm{exc}}+I_{\mathrm{mag}}+I_{\mathrm{mec}}
$$

is the sum of three terms $I_{\text {exc }}, I_{\text {mag }}: \mathcal{B} \rightarrow[0, \infty)$ and $I_{\text {mec }}: \mathcal{B} \rightarrow[0, \infty]$, called the exchange, the magnetostatic, and the mechanical term, respectively, and defined as

$$
\begin{aligned}
I_{\mathrm{exc}}(\mathbf{u}, \mathbf{m}) & :=\int_{\operatorname{im}_{\mathrm{T}}(\mathbf{u}, \Omega)}|D \mathbf{m}(\mathbf{y})|^{2} \mathrm{~d} \mathbf{y}, \quad I_{\mathrm{mag}}(\mathbf{u}, \mathbf{m}):=\frac{1}{2} \int_{\mathbb{R}^{n}}\left|D u_{\mathbf{m}}(\mathbf{y})\right|^{2} \mathrm{~d} \mathbf{y}, \\
I_{\mathrm{mec}}(\mathbf{u}, \mathbf{m}) & :=\int_{\Omega} W(D \mathbf{u}(\mathbf{x}), \mathbf{m}(\mathbf{u}(\mathbf{x}))) \mathrm{d} \mathbf{x} .
\end{aligned}
$$


Here the density $W: \mathbb{R}_{+}^{n \times n} \times \mathbb{S}^{n-1} \rightarrow[0, \infty)$ is a continuous function such that $W(\cdot, \mathbf{m})$ is polyconvex for all $\mathbf{m} \in \mathbb{S}^{n-1}$. We assume that

$$
W(\mathbf{F}, \mathbf{m}) \geq c|\mathbf{F}|^{p}+h(\operatorname{det} \mathbf{F}), \quad \mathbf{F} \in \mathbb{R}_{+}^{n \times n}, \quad \mathbf{m} \in \mathbb{S}^{n-1},
$$

for a constant $c>0$ and a Borel function $h:(0, \infty) \rightarrow[0, \infty)$ for which the limits (8.3) hold.

The scalar function $u_{\mathbf{m}}: \mathbb{R}^{n} \rightarrow \mathbb{R}$ is the magnetostatic potential generated by $\mathbf{m}$, and is a weak solution to Maxwell's equation

$$
\operatorname{div}\left(-D u_{\mathbf{m}}+\chi_{\operatorname{im}_{\mathrm{T}}(\mathbf{u}, \Omega)} \mathbf{m}\right)=0 \quad \text { in } \mathbb{R}^{n},
$$

where the term $\chi_{\mathrm{im}_{\mathrm{T}}(\mathbf{u}, \Omega)} \mathbf{m}$ is the extension of $\mathbf{m}$ to $\mathbb{R}^{n}$ by zero. A precise meaning of a solution of (8.22) requires the introduction of the spaces of B. Levi, otherwise known as the Deny-Lions [22] spaces. We will see that the solution is uniquely determined up to an additive constant; in particular, $D u_{\mathbf{m}}$ is uniquely defined. Call $L^{1,2}\left(\mathbb{R}^{n}\right)$ the space of $u \in L_{\text {loc }}^{2}\left(\mathbb{R}^{n}\right)$ such that $D u \in L^{2}\left(\mathbb{R}^{n}, \mathbb{R}^{n}\right)$. It was proved in $[22$, Cor. 2.1] (see also [58, Sect. 1.1.2]) that $L^{1,2}\left(\mathbb{R}^{n}\right)$ coincides with the set of distributions $u$ in $\mathbb{R}^{n}$ such that $D u \in L^{2}\left(\mathbb{R}^{n}, \mathbb{R}^{n}\right)$. A solution of $(8.22)$ is a $u_{\mathbf{m}} \in L^{1,2}\left(\mathbb{R}^{n}\right)$ such that

$$
\int_{\mathbb{R}^{n}}\left(-D u_{\mathbf{m}}+\chi_{\mathrm{im}_{\mathrm{T}}(\mathbf{u}, \Omega)} \mathbf{m}\right) \cdot D \varphi \mathrm{d} \mathbf{y}=0 \quad \text { for all } \varphi \in L^{1,2}\left(\mathbb{R}^{n}\right) .
$$

We show the existence, uniqueness up to a constant and continuous dependence of the solutions.

Proposition 8.8. For every $\mathbf{f} \in L^{2}\left(\mathbb{R}^{n}, \mathbb{R}^{n}\right)$ there exists $u_{\mathbf{f}} \in L^{1,2}\left(\mathbb{R}^{n}\right)$ such that

$$
\int_{\mathbb{R}^{n}}\left(-D u_{\mathbf{f}}+\mathbf{f}\right) \cdot D \varphi \mathrm{d} \mathbf{y}=0 \quad \text { for all } \varphi \in L^{1,2}\left(\mathbb{R}^{n}\right) .
$$

Moreover, if $u \in L^{1,2}\left(\mathbb{R}^{n}\right)$ satisfies

$$
\int_{\mathbb{R}^{n}}(-D u+\mathbf{f}) \cdot D \varphi \mathrm{d} \mathbf{y}=0 \quad \text { for all } \varphi \in L^{1,2}\left(\mathbb{R}^{n}\right)
$$

then $u=u_{\mathbf{f}}+c$ for some $c \in \mathbb{R}$ and $\|D u\|_{L^{2}\left(\mathbb{R}^{n}, \mathbb{R}^{n}\right)} \leq\|\mathbf{f}\|_{L^{2}\left(\mathbb{R}^{n}, \mathbb{R}^{n}\right)}$.

Proof. Denote by $L^{1,2}\left(\mathbb{R}^{n}\right) / \mathbb{R}$ the quotient space of $L^{1,2}\left(\mathbb{R}^{n}\right)$ with the constant functions. It was shown in $\left[22\right.$, Cor. 1.1] (see also [58, Sect. 1.1.13]) that $L^{1,2}\left(\mathbb{R}^{n}\right) / \mathbb{R}$ is a Hilbert space equipped with the inner product

$$
([u],[\varphi]) \mapsto \int_{\mathbb{R}^{n}} D u \cdot D \varphi
$$

where [.] denotes the equivalence class. We observe that the functional

$$
[\varphi] \mapsto \int_{\mathbb{R}^{n}} \mathbf{f} \cdot D \varphi
$$

is well-defined, linear and continuous in $L^{1,2}\left(\mathbb{R}^{n}\right) / \mathbb{R}$, since

$$
\left|\int_{\mathbb{R}^{n}} \mathbf{f} \cdot D \varphi\right| \leq\|\mathbf{f}\|_{L^{2}\left(\mathbb{R}^{n}, \mathbb{R}^{n}\right)}\|D \varphi\|_{L^{2}\left(\mathbb{R}^{n}, \mathbb{R}^{n}\right)}=\|\mathbf{f}\|_{L^{2}\left(\mathbb{R}^{n}, \mathbb{R}^{n}\right)}\|[\varphi]\|_{L^{1,2}\left(\mathbb{R}^{n}\right) / \mathbb{R}}
$$

As an immediate application of Riesz' representation theorem, we obtain that there exists a unique $\left[u_{\mathbf{f}}\right] \in$ $L^{1,2}\left(\mathbb{R}^{n}\right) / \mathbb{R}$ such that condition (8.23) holds. Moreover, $\left\|\left[u_{\mathbf{f}}\right]\right\|_{L^{1,2}\left(\mathbb{R}^{n}\right) / \mathbb{R}} \leq\|\mathbf{f}\|_{L^{2}\left(\mathbb{R}^{n}, \mathbb{R}^{n}\right)}$. The result follows by translating the properties obtained for $L^{1,2}\left(\mathbb{R}^{n}\right) / \mathbb{R}$ to the corresponding ones in $L^{1,2}\left(\mathbb{R}^{n}\right)$.

With our tools, we are in a position to show the existence of minimizers of $I$ in $\mathcal{B}$. With respect to $[53$, Th. 2.4], we weaken the coercivity condition from $p>n$ to $p>n-1$. Instead, with respect to [69, Th. 4.2], no higher-order gradient of the deformation needs to be considered here, nor is a growth condition from above imposed on $W$. 
Theorem 8.9. Let $\Omega$ be a Lipschitz domain of $\mathbb{R}^{n}, \Gamma$ an $(n-1)$-rectifiable subset of $\partial \Omega$ with $\mathcal{H}^{n-1}(\Gamma)>0$, and $\mathbf{u}_{0}: \Gamma \rightarrow \mathbb{R}^{n}$. Let $p>n-1$, define $\mathcal{B}$ as the set of $(\mathbf{u}, \mathbf{m})$ where $\mathbf{u} \in \mathcal{A}_{p},\left.\mathbf{u}\right|_{\Gamma}=\mathbf{u}_{0}$ and $\mathbf{m} \in$ $W^{1,2}\left(\mathrm{im}_{\mathrm{T}}(\mathbf{u}, \Omega), \mathbb{S}^{n-1}\right)$. Let $W: \mathbb{R}_{+}^{n \times n} \times \mathbb{S}^{n-1} \rightarrow[0, \infty)$ be a continuous function such that $W(\cdot, \mathbf{m})$ is polyconvex for all $\mathbf{m} \in \mathbb{S}^{n-1}$ and equations (8.21) and (8.3) hold for a constant $c>0$ and a Borel function $h:(0, \infty) \rightarrow[0, \infty)$. Define $I$ as in (8.19), (8.20) and (8.22).

If $\mathcal{B} \neq \varnothing$ and $I$ is not identically infinity in $\mathcal{B}$, then $I$ has a minimum in $\mathcal{B}$.

Proof. The proof is similar to that of Theorem 8.2.

Let $\left\{\left(\mathbf{u}_{j}, \mathbf{m}_{j}\right)\right\}_{j \in \mathbb{N}}$ be a minimizing sequence of $I$ in $\mathcal{B}$. The proof of Theorem 8.2 shows that there exists $\mathbf{u} \in \mathcal{A}_{p}$ with $\left.\mathbf{u}\right|_{\Gamma}=\mathbf{u}_{0}$ such that $\mathbf{u}_{j} \rightarrow \mathbf{u}$ in $W^{1, p}\left(\Omega, \mathbb{R}^{n}\right)$. Proposition 7.1 , on the other hand, yields the existence of $\mathbf{m} \in W^{1,2}\left(\operatorname{im}_{\mathrm{T}}(\mathbf{u}, \Omega), \mathbb{S}^{n-1}\right)$ such that $(\mathbf{u}, \mathbf{m}) \in \mathcal{B}$,

$$
\begin{aligned}
& \chi_{\mathrm{im}_{\mathrm{T}}\left(\mathbf{u}_{j}, \Omega\right)} \mathbf{m}_{j} \rightarrow \chi_{\mathrm{im}_{\mathrm{T}}(\mathbf{u}, \Omega)} \mathbf{m} \text { a.e. and in } L^{2}\left(\mathbb{R}^{n}, \mathbb{R}^{n}\right) \\
& \chi_{\mathrm{im}_{\mathrm{T}}\left(\mathbf{u}_{j}, \Omega\right)} D \mathbf{m}_{j} \rightarrow \chi_{\mathrm{im}_{\mathrm{T}}(\mathbf{u}, \Omega)} D \mathbf{m} \quad \text { in } L^{2}\left(\mathbb{R}^{n}, \mathbb{R}^{n \times n}\right) \text { as } j \rightarrow \infty
\end{aligned}
$$

and

$$
I_{\mathrm{exc}}(\mathbf{u}, \mathbf{m}) \leq \liminf _{j \rightarrow \infty} I_{\mathrm{exc}}\left(\mathbf{u}_{j}, \mathbf{m}_{j}\right)
$$

Now, Proposition 7.8 readily yields

$$
I_{\mathrm{mec}}(\mathbf{u}, \mathbf{m}) \leq \liminf _{j \rightarrow \infty} I_{\mathrm{mec}}\left(\mathbf{u}_{j}, \mathbf{m}_{j}\right) .
$$

Finally, let $u_{\mathbf{m}} \in L^{1,2}\left(\mathbb{R}^{n}\right)$ be a solution of $(8.22)$, and recall that $D u_{\mathbf{m}}$ is uniquely determined; analogously for $u_{\mathbf{m}_{j}}$. Owing to Proposition 8.8, convergence (8.24) and the linearity of the Maxwell equation (8.22), we obtain that $D u_{\mathbf{m}_{j}} \rightarrow D u_{\mathbf{m}}$ in $L^{2}\left(\mathbb{R}^{n}, \mathbb{R}^{n}\right)$ as $j \rightarrow \infty$, so

$$
I_{\mathrm{mag}}(\mathbf{u}, \mathbf{m})=\lim _{j \rightarrow \infty} I_{\mathrm{mag}}\left(\mathbf{u}_{j}, \mathbf{m}_{j}\right) .
$$

Thus, $(\mathbf{u}, \mathbf{m})$ is a minimizer of $I$ in $\mathcal{B}$.

Remark 8.10. Recalling Remarks 8.3, 8.4 and 8.7, we can see that the proof of Theorem 8.9 can easily be adapted to cover the case of deformations that are one-to-one a.e. in $\Omega$, as well as the incompressible regime and an inhomogeneous energy $W$.

\section{Acknowledgements}

We thank S. Hencl for pointing us out that condition INV implies the differentiability a.e. D.H. has been supported by FONDECYT project 1150038 of the Chilean Ministry of Education and by the Millennium Nucleus Center for Analysis of PDE NC130017 of the Chilean Ministry of Economy. C.M.-C. has been supported by Project MTM2014-57769-C3-1-P and the "Ramón y Cajal" programme RYC-2010-06125 of the Spanish Ministry of Economy and Competitivity, and the ERC Starting grant no. 307179.

\section{References}

[1] V. Agostiniani And A. DeSimone, $\Gamma$-convergence of energies for nematic elastomers in the small strain limit, Contin. Mech. Thermodyn., 23 (2011), pp. 257-274.

[2] L. Ambrosio, N. Fusco, And D. Pallara, Functions of bounded variation and free discontinuity problems, Oxford University Press, New York, 2000.

[3] N. Ansini AND A. Garroni, Г-convergence of functionals on divergence-free fields, ESAIM Control Optim. Calc. Var., 13 (2007), pp. 809-828.

[4] K. Astala, T. Iwaniec, And G. Martin, Elliptic partial differential equations and quasiconformal mappings in the plane, vol. 48 of Princeton Mathematical Series, Princeton University Press, Princeton, NJ, 2009. 
[5] J. M. BALL, Constitutive inequalities and existence theorems in nonlinear elastostatics, in Nonlinear analysis and mechanics: Heriot-Watt Symposium (Edinburgh, 1976), Vol. I, Pitman, London, 1977, pp. 187-241. Res. Notes in Math., No. 17.

[6] - Convexity conditions and existence theorems in nonlinear elasticity, Arch. Rational Mech. Anal., 63 (1977), pp. 337-403.

[7] — Global invertibility of Sobolev functions and the interpenetration of matter, Proc. Roy. Soc. Edinburgh Sect. A, 88 (1981), pp. 315-328.

[8] J. M. Ball, J. C. Currie, And P. J. Olver, Null Lagrangians, weak continuity, and variational problems of arbitrary order, J. Funct. Anal., 41 (1981), pp. 135-174.

[9] J. M. Ball and A. Majumdar, Nematic liquid crystals: From Maier-Saupe to a continuum theory, Mol. Cryst. Liq. Cryst., 525 (2009), pp. 1-11.

[10] J. M. Ball And F. Murat, Remarks on Chacon's biting lemma, Proc. Amer. Math. Soc., 107 (1989), pp. 655663.

[11] M. BARchiesi And A. DeSimone, Frank energy for nematic elastomers: a nonlinear model, ESAIM Control Optim. Calc. Var., 21 (2015), pp. 372-377.

[12] N. Bourbaki, Elements of mathematics. General topology. Part 1, Hermann, Paris, 1966.

[13] J. K. Brooks And R. V. Chacon, Continuity and compactness of measures, Adv. in Math., 37 (1980), pp. 1626.

[14] M. C. Calderer, C. A. Garavito Garzón, and B. Yan, A Landau-de Gennes theory of liquid crystal elastomers, Discrete Contin. Dyn. Syst. Ser. S, 8 (2015), pp. 283-302.

[15] P. G. Ciarlet And J. NeČAs, Injectivity and self-contact in nonlinear elasticity, Arch. Rational Mech. Anal., 97 (1987), pp. 171-188.

[16] S. Conti And C. De Lellis, Some remarks on the theory of elasticity for compressible Neohookean materials, Ann. Sc. Norm. Super. Pisa Cl. Sci. (5), 2 (2003), pp. 521-549.

[17] B. Dacorogna, Direct methods in the calculus of variations, vol. 78 of Applied Mathematical Sciences, Springer, New York, second ed., 2008.

[18] B. Dacorogna And I. Fonseca, A minimization problem involving variation of the domain, Comm. Pure Appl. Math., 45 (1992), pp. 871-897.

[19] P. De Gennes and J. J. Prost, The physics of liquid crystals, Oxford University Press, USA, 1993.

[20] C. De Lellis And F. Ghiraldin, An extension of the identity Det = det, C. R. Math. Acad. Sci. Paris, 348 (2010), pp. 973-976.

[21] K. Deimling, Nonlinear functional analysis, Springer, Berlin, 1985.

[22] J. Deny and J. L. Lions, Les espaces du type de Beppo Levi, Ann. Inst. Fourier, Grenoble, 5 (1954), pp. 305-370.

[23] A. DeSimone, Energy minimizers for large ferromagnetic bodies, Arch. Rational Mech. Anal., 125 (1993), pp. 99143.

[24] A. DeSimone And G. Dolzmann, Existence of minimizers for a variational problem in two-dimensional nonlinear magnetoelasticity, Arch. Rational Mech. Anal., 144 (1998), pp. 107-120.

[25] A. DeSimone And R. D. James, A constrained theory of magnetoelasticity, J. Mech. Phys. Solids, 50 (2002), pp. $283-320$.

[26] A. DeSimone And L. Teresi, Elastic energies for nematic elastomers, Eur. Phys. J. E, 29 (2009), pp. $191-204$.

[27] J. Dieudonné, Treatise on analysis. Vol. III, Academic Press, New York, 1972.

[28] L. D'Onofrio, S. Hencl, J. Malý, and R. Schiattarella, Note on Lusin (N) condition and the distributional determinant, J. Math. Anal. Appl., 439 (2016), pp. 171-182.

[29] L. C. Evans And R. F. Gariepy, Measure theory and fine properties of functions, CRC Press, Boca Raton, FL, 1992.

[30] H. Federer, Geometric measure theory, Springer, New York, 1969.

[31] I. Fonseca And W. Gangbo, Degree theory in analysis and applications, Oxford University Press, New York, 1995. 
[32] _ Local invertibility of Sobolev functions, SIAM J. Math. Anal., 26 (1995), pp. 280-304.

[33] I. Fonseca And G. LeOni, Modern methods in the calculus of variations: $L^{p}$ spaces, Springer Monographs in Mathematics, Springer, New York, 2007.

[34] I. Fonseca And S. MÜller, A-quasiconvexity, lower semicontinuity, and Young measures, SIAM J. Math. Anal., 30 (1999), pp. 1355-1390.

[35] I. Fonseca And G. PARry, Equilibrium configurations of defective crystals, Arch. Rational Mech. Anal., 120 (1992), pp. 245-283.

[36] — On a class of invariant functionals, Proc. Roy. Soc. London Ser. A, 436 (1992), pp. 317-329.

[37] F. R. Gantmacher, The theory of matrices. Vol. 1, Chelsea Publishing Co., New York, 1959.

[38] M. Giaquinta, G. Modica, And J. SoučEK, Cartesian currents in the calculus of variations. I, Springer-Verlag, Berlin, 1998.

[39] — Cartesian currents in the calculus of variations. II, Springer-Verlag, Berlin, 1998.

[40] C. Goffman And W. P. Ziemer, Higher dimensional mappings for which the area formula holds, Ann. of Math. (2), 92 (1970), pp. 482-488.

[41] P. Hajlasz And J. Malý, Approximation in Sobolev spaces of nonlinear expressions involving the gradient, Ark. Mat., 40 (2002), pp. 245-274.

[42] D. Henao AND C. Mora-Corral, Invertibility and weak continuity of the determinant for the modelling of cavitation and fracture in nonlinear elasticity, Arch. Rational Mech. Anal, 197 (2010), pp. 619-655.

[43] — Fracture surfaces and the regularity of inverses for BV deformations, Arch. Rational Mech. Anal., 201 (2011), pp. 575-629.

[44] _ Lusin's condition and the distributional determinant for deformations with finite energy, Adv. Calc. Var., 5 (2012), pp. 355-409.

[45] — Regularity of inverses of Sobolev deformations with finite surface energy, J. Funct. Anal., 268 (2015), pp. 2356-2378.

[46] D. Henao, C. Mora-Corral, And X. XU, $\Gamma$-convergence approximation of fracture and cavitation in nonlinear elasticity, Arch. Rational Mech. Anal., 216 (2015), pp. 813-879.

[47] S. Hencl And P. Koskela, Lectures on mappings of finite distortion, vol. 2096 of Lecture Notes in Mathematics, Springer, Cham, 2014.

[48] T. Iwaniec and J. Onninen, Hyperelastic deformations of smallest total energy, Arch. Rational Mech. Anal., 194 (2009), pp. 927-986.

[49] R. D. James And D. Kinderlehrer, Frustration in ferromagnetic materials, Contin. Mech. Thermodyn., 2 (1990), pp. 215-239.

[50] — Theory of magnetostriction with application to $T b_{x} D y_{1-x} F e_{2}$, Phil. Mag. B, 68 (1993), pp. 237-274.

[51] L. V. Kovalev and J. Onninen, On invertibility of Sobolev mappings, J. Reine Angew. Math., 656 (2011), pp. $1-16$.

[52] L. V. Kovalev, J. Onninen, And K. Rajala, Invertibility of Sobolev mappings under minimal hypotheses, Ann. Inst. H. Poincaré Anal. Non Linéaire, 27 (2010), pp. 517-528.

[53] M. KRuŽÍK, U. Stefanelli, And J. Zeman, Existence results for incompressible magnetoelasticity, Discrete Contin. Dyn. Syst., 35 (2015), pp. 2615-2623.

[54] J. G. Llavona, Approximation of continuously differentiable functions, vol. 130 of North-Holland Mathematics Studies, North-Holland Publishing Co., Amsterdam, 1986.

[55] A. Majumdar, Equilibrium order parameters of nematic liquid crystals in the Landau-de Gennes theory, European J. Appl. Math., 21 (2010), pp. 181-203.

[56] J. J. Manfredi, Weakly monotone functions, J. Geom. Anal., 4 (1994), pp. 393-402.

[57] M. Marcus And V. J. Mizel, Transformations by functions in Sobolev spaces and lower semicontinuity for parametric variational problems, Bull. Amer. Math. Soc., 79 (1973), pp. 790-795.

[58] V. MAZ'YA, Sobolev spaces with applications to elliptic partial differential equations, vol. 342 of Grundlehren der Mathematischen Wissenschaften, Springer, Heidelberg, 2nd ed., 2011. 
[59] S. MülleR, Weak continuity of determinants and nonlinear elasticity, C. R. Acad. Sci. Paris Sér. I Math., 307 (1988), pp. 501-506.

[60] S. Müller, Det = det. A remark on the distributional determinant, C. R. Acad. Sci. Paris Sér. I Math., 311 (1990), pp. 13-17.

[61] S. Müller And S. J. SpeCtor, An existence theory for nonlinear elasticity that allows for cavitation, Arch. Rational Mech. Anal., 131 (1995), pp. 1-66.

[62] S. Müller, S. J. Spector, And Q. TANG, Invertibility and a topological property of Sobolev maps, SIAM J. Math. Anal., 27 (1996), pp. 959-976.

[63] S. Müller, Q. TANG, AND B. S. YAN, On a new class of elastic deformations not allowing for cavitation, Ann. Inst. H. Poincaré Anal. Non Linéaire, 11 (1994), pp. 217-243.

[64] F. Murat, Compacité par compensation: condition nécessaire et suffisante de continuité faible sous une hypothèse de rang constant, Ann. Scuola Norm. Sup. Pisa Cl. Sci. (4), 8 (1981), pp. 69-102.

[65] M. Palombaro, Rank- $(n-1)$ convexity and quasiconvexity for divergence free fields, Adv. Calc. Var., 3 (2010), pp. 279-285.

[66] M. Palombaro And V. P. Smyshlyaev, Relaxation of three solenoidal wells and characterization of extremal three-phase H-measures, Arch. Rational Mech. Anal., 194 (2009), pp. 775-822.

[67] S. P. Ponomarev, Property $N$ of homeomorphisms of the class $W^{1, p}$, Sib. Math. J., 28 (1987), pp. 291-298.

[68] Y. G. Reshetnyak, Space mappings with bounded distortion, vol. 73 of Translations of Mathematical Monographs, American Mathematical Society, Providence, RI, 1989.

[69] P. RyBKA AND M. Luskin, Existence of energy minimizers for magnetostrictive materials, SIAM J. Math. Anal., 36 (2005), pp. 2004-2019.

[70] V. ŠveráK, Regularity properties of deformations with finite energy, Arch. Rational Mech. Anal., 100 (1988), pp. $105-127$.

[71] Q. TAnG, Almost-everywhere injectivity in nonlinear elasticity, Proc. Roy. Soc. Edinburgh Sect. A, 109 (1988), pp. 79-95.

[72] S. K. Vodop'Yanov, Topological and geometrical properties of mappings with summable Jacobian in Sobolev classes. I, Siberian Math. J., 41 (2000), pp. 19-39.

[73] S. K. VOdOP'YANOV AND V. M. GOL'DŠTEǏN, Quasiconformal mappings, and spaces of functions with first generalized derivatives, Sibirsk. Mat. Ž., 17 (1976), pp. 515-531, 715.

[74] M. Warner and E. Terentjev, Liquid Crystal Elastomers, Clarendon Press, Oxford, 2007.

[75] W. P. Ziemer, Weakly differentiable functions, Springer, New York, 1989. 